\title{
Synthesis of 7-chloroquinoline derivatives using mixed lithium-magnesium reagents
}

\author{
Valter E. Murie ${ }^{\mathrm{a}}$, Paula V. Nicolino ${ }^{\mathrm{a}}$, Thiago dos Santos ${ }^{\mathrm{a}}$, Guido Gambacorta ${ }^{\mathrm{b}}$, Rodolfo \\ H. V. Nishimura ${ }^{a}$, Icaro S. Perovani ${ }^{\text {, }}$, Luciana C. Furtado ${ }^{\text {d }}$, Leticia V. Costa-Lotufo ${ }^{d}$, \\ Anderson Moraes de Oliveira ${ }^{\mathrm{c}}$, Ricardo Vessecchi ${ }^{\mathrm{c}}$, Ian R. Baxendale* b and Giuliano \\ C. Clososki*a,c \\ ${ }^{\text {a }}$ Departamento de Ciências BioMoleculares, Faculdade de Ciências Farmacêuticas de Ribeirão Preto, \\ Universidade de São Paulo, Av. do Café S/N, Ribeirão Preto, 14040-903, Brazil \\ ${ }^{b}$ Department of Chemistry, Durham University, South Road, Durham, DH1 3LE, United Kingdom \\ ${ }^{\mathrm{c}}$ Departamento de Química, Faculdade de Filosofia Ciências e Letras de Ribeirão Preto, Universidade de \\ São Paulo, Av. Bandeirantes 3900, Ribeirão Preto, 14040-901, Brazil

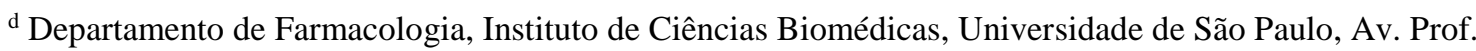 \\ Lineu Prestes 1524, São Paulo, 05508-900, Brazil.
}

*E-mail: i.r.baxendale@durham.ac.uk; gclososki@usp.br

\section{List of Contents}

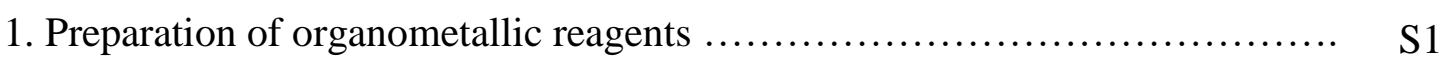

2. Survey of reaction conditions for iodo-magnesium exchange reaction ........ S2

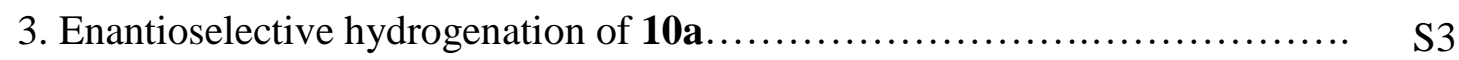

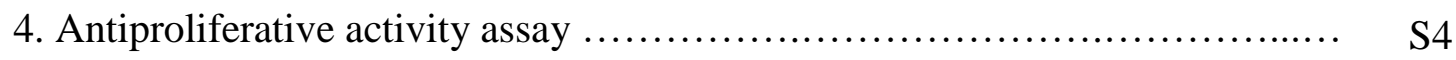

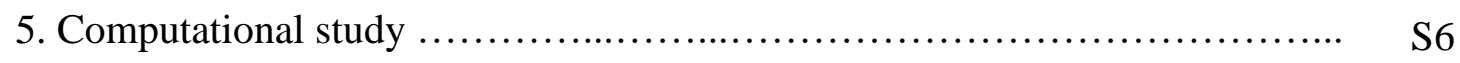

6. Flow configurations. .......................................... S16

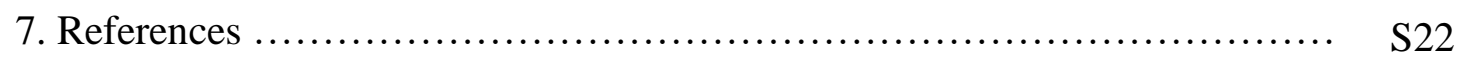

8. ${ }^{1} \mathrm{H}$ NMR and ${ }^{13} \mathrm{C}$ NMR spectra for all compounds ..................... S23 


\section{Preparation of organometallic reagents}

\section{n-Butyllithium titration}

In a dry nitrogen-flushed round-bottom flask $(10 \mathrm{~mL})$ containing a solution of 1,10 phenantroline (small amount) and $n$-BuLi (1.0 mL, 2.5 mol. $\mathrm{L}^{-1}$ in hexanes) in anhydrous THF (2.0 mL) at $0{ }^{\circ} \mathrm{C}$, it was added dropwise isopropanol (freshly distilled) until the solution color turned from dark red to yellow. This procedure was performed in triplicate.

\section{$i$-PrMgCl-LiCl titration}

In a dry nitrogen-flushed round-bottom flask $(10 \mathrm{~mL})$ containing a solution of iodine (127 $\mathrm{mg}, 0.5 \mathrm{mmol})$ in anhydrous THF, it was added dropwise $i$ - $\mathrm{PrMgCl} \mathrm{LiCl}\left(1.3 \mathrm{~mol} . \mathrm{L}^{-1}\right.$ in THF) until the reaction mixture became colorless. This procedure was performed in triplicate.

\section{Preparation of $\mathrm{ZnCl}_{2}$ anhydrous solution in $\mathrm{THF}$}

In a dry Schlenk flask $(50 \mathrm{~mL})$ containing $\mathrm{ZnCl}_{2}(2.7257 \mathrm{~g}, 20 \mathrm{mmol})$ and a magnetic bar was dried under high vacuum at $140{ }^{\circ} \mathrm{C}$ for $10 \mathrm{~h}$. After this period, the flask was cooled and the vacuum was broken under nitrogen atmosphere. The mass of zinc chloride was verified. Then, dry THF $(20 \mathrm{~mL})$ was added and the mixture was stirred until the complete solubilization of the salt. The solution was stored under nitrogen atmosphere and low temperature $\left(\approx 5^{\circ} \mathrm{C}\right)$.

\section{Preparation of TMPMgCl-LiCl anhydrous solution in THF}

In a dry and nitrogen-flushed Schlenk flask $(25 \mathrm{~mL})$ equipped with magnetic bar and rubber septum was charged with $i$-PrMgCl $\mathrm{LiCl}\left(16.7 \mathrm{~mL}, 20 \mathrm{mmol}, 1.2 \mathrm{~mol} . \mathrm{L}^{-1}\right.$ in THF), 2,2,6,6-tetramethylpiperidine $(3.74 \mathrm{~mL}, 22 \mathrm{mmol})$ was added dropwise at $0{ }^{\circ} \mathrm{C}$. The mixture was stirred for $48 \mathrm{~h}$.

\section{TMPMgCl·LiCl titration}

In a dry nitrogen-flushed round bottom flask $(10 \mathrm{~mL})$ containing benzoic acid $(32 \mathrm{mg}$, $0.26 \mathrm{mmol}$ ) and 4-(phenylazo)diphenylamine (small amount) as an indicator in anhydrous THF ( $1 \mathrm{~mL})$, the TMPMgCl $\mathrm{LiCl}$ solution was added dropwise until the reaction medium color changed from orange to rouge. 


\section{Survey of reaction conditions for iodo-magnesium exchange reaction}<smiles>Clc1ccc2c(I)ccnc2c1</smiles>

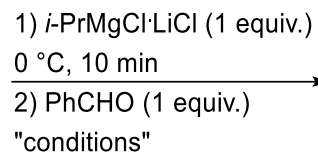<smiles>Clc1ccc2cccnc2c1</smiles>

$7 a$<smiles>OC(c1ccccc1)c1ccnc2cc(Cl)ccc12</smiles>

9a<smiles>O=C(c1ccccc1)c1ccnc2cc(Cl)ccc12</smiles>

$10 \mathrm{a}$

Scheme S1. Halogen-metal exchange reaction between 7-chloro-4-iodoquinoline and Turbo Grignard followed by the reaction with benzaldehyde as an electrophile.

Table S1. Methodological study of iodo-magnesium exchange reaction using benzaldehyde as an electrophile.

\begin{tabular}{|c|c|c|c|c|c|}
\hline \multirow{2}{*}{ Entry } & \multirow{2}{*}{ Temperature $\left({ }^{\circ} \mathrm{C}\right)$} & \multirow{2}{*}{ Time (h) } & \multicolumn{3}{|c|}{ Chromatographic composition $(\%)^{a}$} \\
\hline & & & $7 \mathbf{a}$ & $9 \mathbf{a}$ & $10 \mathbf{a}$ \\
\hline 1 & 0 to 20 & 12 & - & 82 & 18 \\
\hline 2 & 25 & 12 & 5 & 80 & 15 \\
\hline $3^{c}$ & 0 to 20 & 12 & 6 & 82 & 12 \\
\hline 4 & 25 & 1,5 & 9 & 71 & 20 \\
\hline 5 & -20 to 15 & 1,5 & 2 & 85 & 13 \\
\hline 6 & 0 to 15 & 1,5 & 2 & 89 & 9 \\
\hline 7 & 0 to 15 & 1 & 3 & 91 & 6 \\
\hline $8^{b}$ & 0 & 0,25 & 6 & 80 & 9 \\
\hline $9^{c}$ & 0 & 0,5 & 5 & 82 & 6 \\
\hline 10 & 0 & 1 & - & 93 & 7 \\
\hline
\end{tabular}




\section{compound}

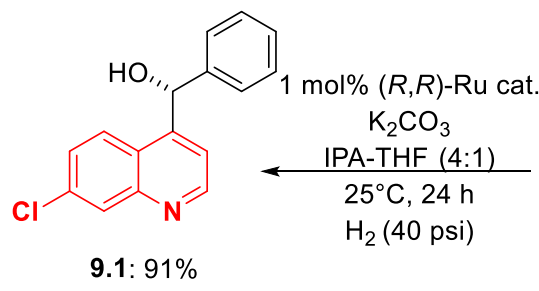

$(99.4 \%$ ef)

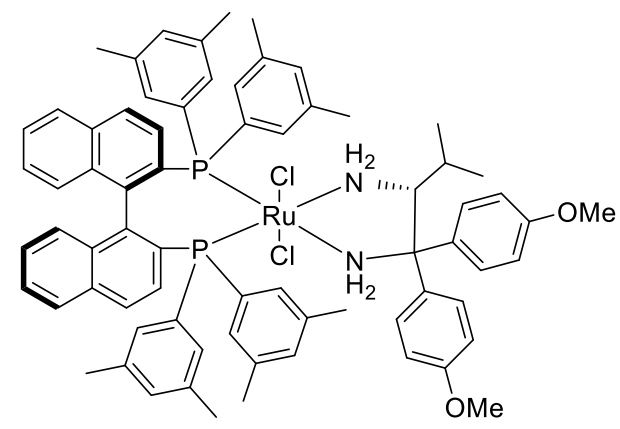

trans- $\mathrm{RuCl}_{2}[(R)$-xylbinap $][(R)$-daipen] $(\mathbf{C 2})$<smiles>O=C(c1ccccc1)c1ccnc2cc(Cl)ccc12</smiles>

$10 \mathrm{a}$

$1 \mathrm{~mol} \%(S, S)-\mathrm{Ru}$ cat. $\mathrm{K}_{2} \mathrm{CO}_{3}$ $\underset{\text { IPA-THF }(4: 1)}{25^{\circ} \mathrm{C}, 24 \mathrm{~h}}$ $\mathrm{H}_{2}(40 \mathrm{psi})$

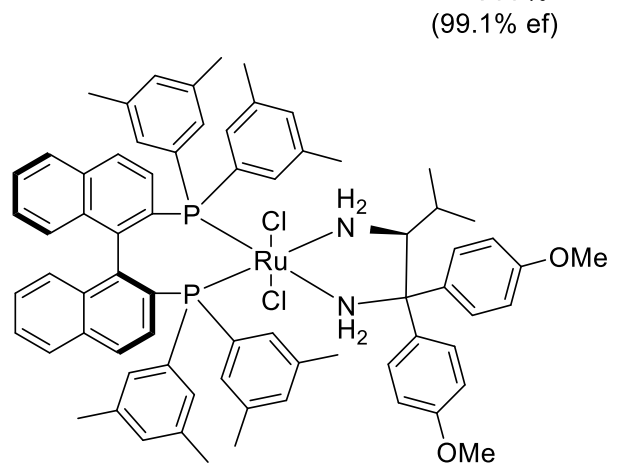

trans- $\mathrm{RuCl}_{2}[(S)$-xylbinap][(S)-daipen] (C1)

Scheme S2. Enantioselective reduction using ruthenium catalysts

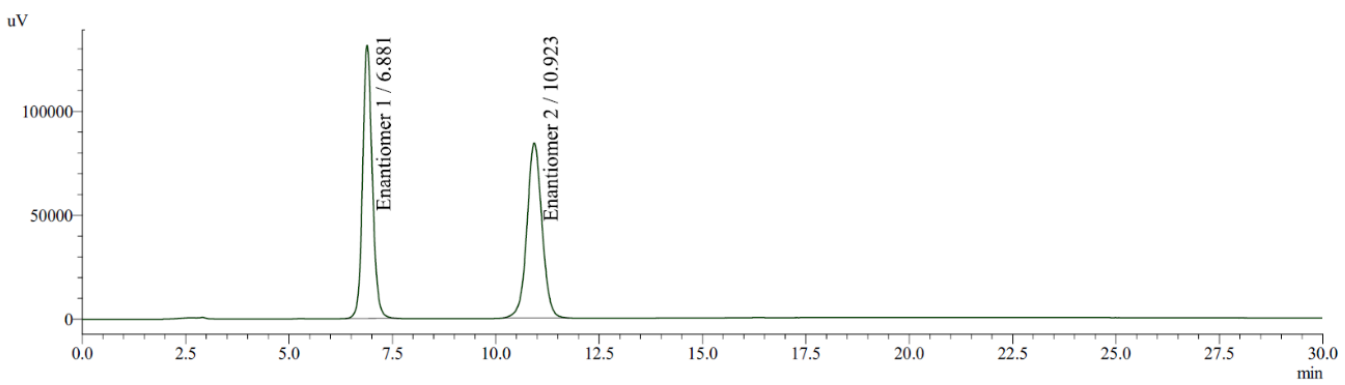

Figure S1. Enantioseparation of compound 10a. Chromatographic conditions: Chiralpak AD-H $(150 \times 4.6 \mathrm{~mm}, 5 \mu \mathrm{m})$ column and methanol $(100 \%)$ as mobile phase. The column temperature, flow rate and injection volume were set at $30{ }^{\circ} \mathrm{C}, 0.4 \mathrm{~mL} \mathrm{~min}^{-1}$, and $10 \mu \mathrm{L}$, respectively. Detection was carried out at $278 \mathrm{~nm}$. 


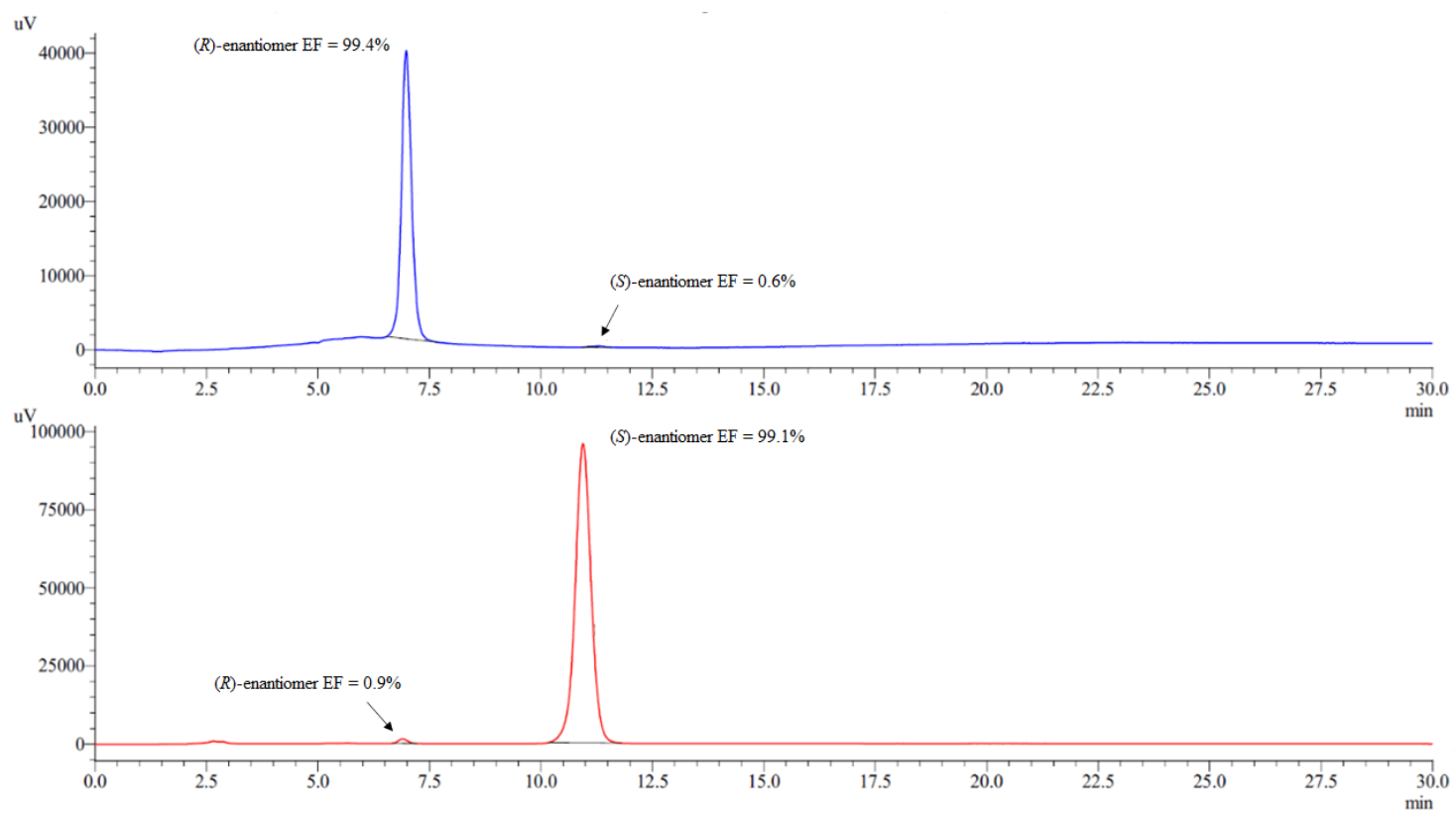

Figure S2. Chromatograms obtained after the enantioselective hydrogenation of compound 10a. The enantiomeric fraction $(\mathrm{EF})$ obtained for each enantiomer is displayed in the chromatogram. The blue chromatogram represents the $(R)$ enantiomer obtained when using the catalyst trans- $\operatorname{RuCl}_{2}[(R)$-xylbinap $][(R)$-daipen $]$ while the red chromatogram represents the $(S)$ enantiomer obtained when using the catalyst trans$\operatorname{RuCl}_{2}[(S)$-xylbinap $][(S)$-daipen $]$

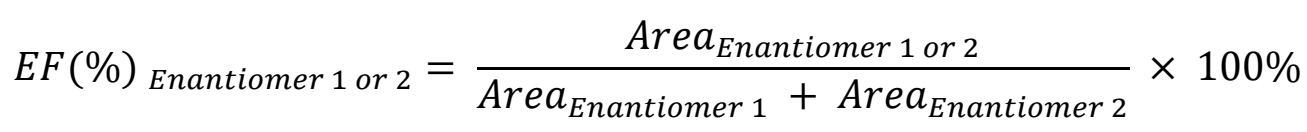

Equation S1. Equation for the calculation of each enantiomeric fraction.

\section{Antiproliferative activity assay}

\subsection{Screening using the cancer cell lines: human oligodendroglioma (HOB), colon} adenocarcinoma (HCT-116), and human glioblastoma (T98G): All the compounds were prepared at $10 \mathrm{mM}$ stock solutions in DMSO. The screening evaluated the capability of these molecules in inhibiting the cancer cells growth at least $75 \%$ by colorimetric MTT (3-(4,5-dimethylthiazol-2-yl)-2,5-diphenyltetrazolium bromide) assay. DMSO and doxorubicin were used as negative and positive control, respectively. The cancer cells were seeded into a 96-well microplate at different densities, $3 \times 10^{4}$ cells $/ \mathrm{mL}$ for HOG and T98G, and $5 \times 10^{4}$ cells $/ \mathrm{mL}$ for HCT116, $24 \mathrm{~h}$ before the treatment. Cells were treated with $50 \mu \mathrm{M}$ of each compound and incubated for $72 \mathrm{~h}$. The supernatant was substituted 
by culture medium $(150 \mu \mathrm{L})$ containing MTT $(0.5 \mathrm{mg} / \mathrm{mL})$ and the cells were incubated for an additional $3 \mathrm{~h}$. Then, the supernatant was removed and the microplate was dried at least $3 \mathrm{~h}$. The precipitated formazan was dissolved in DMSO $(150 \mu \mathrm{L})$ and the absorbance intensity was measured at $570 \mathrm{~nm}$ using a multi-well scanning spectrophotometer (Multiskan FC, Fisher Scientific, USA). ${ }^{1}$
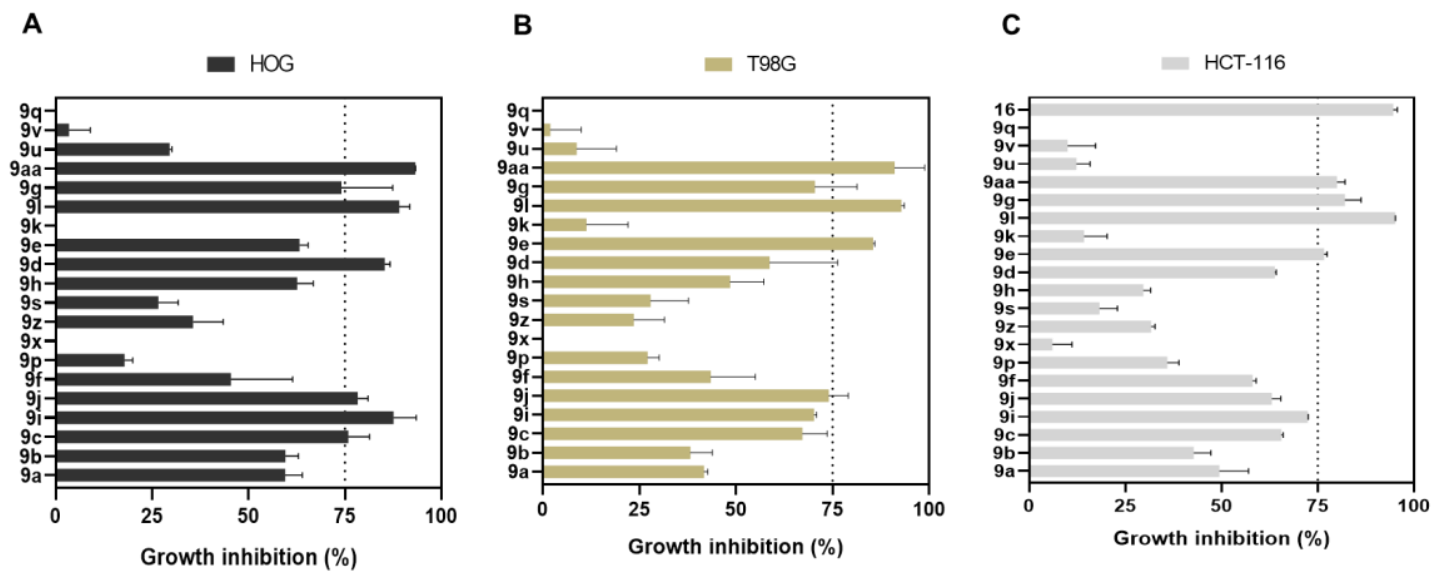

Figure S3. Screening using human oligodendroglioma cells (HOB) Screening of compounds in cancer cells: HOG (A), T98G (B), and HCT116 (C). Using HOG and T98G cancer cells, compounds $9 \mathbf{j}, \mathbf{9 1}$ and 9 aa were capable of inhibiting $>75 \%$ of the tumor growth at $50 \mathrm{mM}$ for both cell types. Considering the same inhibition percentage, 9c, 9d, 9g and $9 \mathbf{i}$ have selectively inhibited HOG cancer cells whilst $9 \mathbf{e}$ was only active against T98G cancer cells. For HCT116 cancer cells, compounds 9e, 9g, 9l, 9aa and 16 were capable of inhibiting more than $75 \%$ of cell growth at $50 \mu \mathrm{M}$.

\subsection{IC50 values determination: human lung carcinoma (A549) and human colorectal} carcinoma (HCT116). In a second set of experiments, selected compounds 9e, 9g, 9l, 9aa, and 16 were tested against A549 and HCT116 cells. (Methodology described in detail in the manuscript). 
Table S2. Compounds 9e, 9g, 91, 9aa, and 16 were evaluated using MTT assay after $72 \mathrm{~h}$ of exposition against A549, and HCT116 cells to assess $\mathrm{IC}_{50}$ values. A549 (n=4), HCT116 $(n=2)$.

\begin{tabular}{cccccccc}
\hline & & \multicolumn{2}{c}{$\mathrm{IC}_{50}(\mu \mathrm{M})$} & \multicolumn{2}{c}{$95 \% \mathrm{CI}$} & \multicolumn{2}{c}{$\mathrm{R}^{2}$} \\
\cline { 3 - 8 } Entry & Compound & $\mathrm{A549}$ & $\mathrm{HCT} 116$ & A549 & HCT116 & A549 & HCT116 \\
\hline 1 & $\mathbf{9 e}$ & 16.3 & 21.94 & $13.86-19.22$ & $16.82-28.77$ & 0.93 & 0.96 \\
2 & $\mathbf{9 g}$ & 19.13 & 20.09 & $13.66-17.44$ & $13.79-15.67$ & 0.97 & 0.99 \\
3 & $\mathbf{9 1}$ & 15.43 & 14.7 & $16.30-22.49$ & $18.20-22.19$ & 0.95 & 0.99 \\
4 & $\mathbf{9 a a}$ & 12.51 & 10.76 & $9.51-16.45$ & $8.11-14.49$ & 0.89 & 0.97 \\
5 & $\mathbf{1 6}$ & 14.95 & 11.03 & $12.21-18.65$ & $8.24-14.39$ & 0.89 & 0.96 \\
6 & Doxorubicin & 0.05 & 1.568 & $0.04-0.06$ & $0.75-3.35$ & 0.96 & 0.97 \\
\hline
\end{tabular}

\section{Computational study: $\mathrm{p} K_{\mathrm{a}}$ values and complexation with $\mathrm{ZnCl}_{2}$}
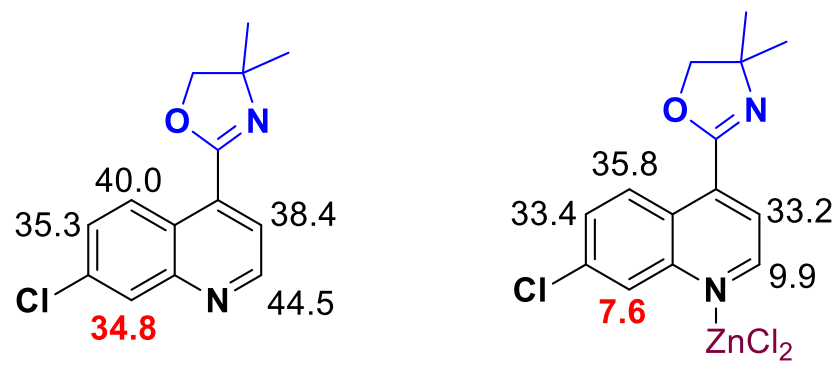

Figure S4. $\mathrm{p} K_{\mathrm{a}}$ values of compound $10 \mathrm{~g}$ and complexed with $\mathrm{ZnCl}_{2}$.

Geometries were optimized using B3PW91/6-31G(d). pKa values were computed using a hypothetical reaction with pyridine as reference compound in THF through singlepoint calculations performed in B3LYP/6-311++G(d,p) model using PCM method. All calculations were done in Gaussian 03 software.<smiles>CC1(C)COC(c2ccnc3cc(Cl)ccc23)=N1</smiles>

Figure S5. Example of isodesmic reaction for $\mathrm{p} K_{\mathrm{a}}$ calculations.

For complexes with $\mathrm{ZnCl}_{2}$, the complexation with $\mathrm{N}$ atoms were performed and the results suggest that difference of Gibbs energies in solution (THF) is $-2.97 \mathrm{kcal} / \mathrm{mol}$ for complexation in quinoline nitrogen atom (PCM/B3LYP/6-31++G(d,p)//B3PW91/6- 
$31 \mathrm{G}(\mathrm{d}))$. Thus, only this possibily was tested and the $\mathrm{p} K_{\mathrm{a}}$ values was estimated for complexe at quinoline nitrogen.<smiles>c1ccncc1</smiles><smiles>c1ccncc1</smiles><smiles>CC1(C)COC(c2ccnc3cc(Cl)ccc23)=N1</smiles>

$-1185.888926$<smiles></smiles>

$-1185.902266$<smiles>CC1(C)COC(c2ccnc3cc(Cl)ccc23)=N1</smiles>

$-1185.898815$<smiles>CC1(C)COC(c2ccnc3cc(Cl)ccc23)=N1</smiles>

$-1186.457788$<smiles></smiles>

$-3885.913726$

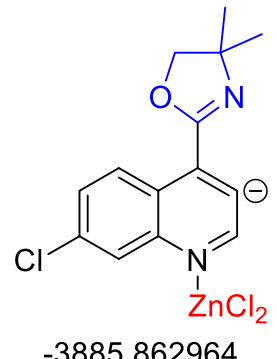

$-3885.862964$<smiles></smiles><smiles>CC1(C)COC(c2ccnc3cc(Cl)ccc23)=N1</smiles>

$-1185.910219$<smiles>CC1(C)COC(c2ccnc3cc(Cl)c(Cl)cc23)=N1</smiles><smiles></smiles>

$-3886.402491$<smiles></smiles>

$-3885.918671$

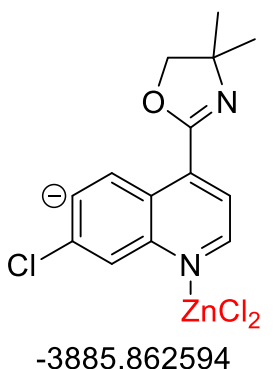

Figure S6. Gibbs energies in solution for studied species obtained at PCM(THF)/B3LYP/6-311++G(d,p)//B3PW91/6-31G(d) level. 
Table S3. Equilibrium geometries for neutral and deprotonated obtained in B3PW91/6-31G(d)

\begin{tabular}{|c|c|c|c|c|}
\hline Structure & Atom & $\mathbf{X}$ & $\mathbf{Y}$ & $\mathbf{Z}$ \\
\hline & $\mathrm{C}$ & -2.42488 & -1.55922 & 0.01768 \\
\hline & $\mathrm{C}$ & -3.48777 & -0.62999 & 0.02204 \\
\hline & $\mathrm{C}$ & -3.24834 & 0.71960 & 0.01341 \\
\hline & $\mathrm{C}$ & -1.91385 & 1.19967 & -0.00058 \\
\hline & $\mathrm{C}$ & -0.81884 & 0.27197 & -0.01538 \\
\hline & $\mathrm{C}$ & -1.12457 & -1.11517 & -0.00101 \\
\hline & $\mathrm{N}$ & -1.75919 & 2.55065 & 0.00664 \\
\hline & $\mathrm{C}$ & -0.52965 & 3.01805 & 0.00888 \\
\hline & $\mathrm{C}$ & 0.62203 & 2.20939 & -0.00531 \\
\hline & $\mathrm{C}$ & 0.49730 & 0.83298 & -0.02315 \\
\hline & $\mathrm{C}$ & 1.76034 & 0.06151 & -0.03454 \\
\hline & $\mathrm{N}$ & 2.89448 & 0.55848 & 0.27167 \\
\hline & $\mathrm{C}$ & 3.90682 & -0.48981 & 0.07530 \\
\hline & $\mathrm{C}$ & 3.06119 & -1.74022 & -0.29701 \\
\hline & $\mathrm{O}$ & 1.71560 & -1.24261 & -0.42492 \\
\hline & $\mathrm{C}$ & 4.69361 & -0.69137 & 1.37104 \\
\hline & $\mathrm{C}$ & 4.83762 & -0.06599 & -1.06542 \\
\hline & $\mathrm{H}$ & -2.64407 & -2.62212 & 0.02905 \\
\hline & $\mathrm{H}$ & -4.05058 & 1.44893 & 0.02181 \\
\hline & $\mathrm{H}$ & -0.31711 & -1.83522 & -0.00980 \\
\hline & $\mathrm{H}$ & -0.42408 & 4.10253 & 0.01766 \\
\hline & $\mathrm{H}$ & 1.61174 & 2.65282 & -0.00351 \\
\hline & $\mathrm{H}$ & 3.06618 & -2.50897 & 0.48392 \\
\hline & $\mathrm{H}$ & 3.34423 & -2.19521 & -1.25071 \\
\hline & $\mathrm{H}$ & 5.43928 & -1.48708 & 1.25426 \\
\hline & $\mathrm{H}$ & 4.02361 & -0.96180 & 2.19413 \\
\hline & $\mathrm{H}$ & 5.21375 & 0.23132 & 1.64645 \\
\hline & $\mathrm{H}$ & 5.61305 & -0.82272 & -1.23581 \\
\hline & $\mathrm{H}$ & 5.32659 & 0.88241 & -0.82200 \\
\hline & $\mathrm{H}$ & 4.27619 & 0.06965 & -1.99631 \\
\hline & $\mathrm{Cl}$ & -5.12569 & -1.22976 & 0.04272 \\
\hline \multirow[t]{12}{*}{ Structure } & atom & $\mathbf{X}$ & $\mathbf{Y}$ & $\mathbf{Z}$ \\
\hline & $\mathrm{C}$ & 2.47086 & -1.52716 & 0.06233 \\
\hline & $\mathrm{C}$ & 3.50448 & -0.58583 & 0.01154 \\
\hline & $\mathrm{C}$ & 3.23981 & 0.76501 & -0.05933 \\
\hline & $\mathrm{C}$ & 1.90767 & 1.22754 & -0.07619 \\
\hline & $\mathrm{C}$ & 0.82138 & 0.28803 & 0.00911 \\
\hline & $\mathrm{C}$ & 1.15710 & -1.08533 & 0.05445 \\
\hline & $\mathrm{N}$ & 1.68105 & 2.56903 & -0.33507 \\
\hline & $\mathrm{C}$ & 0.49130 & 3.03474 & -0.00681 \\
\hline & $\mathrm{C}$ & -0.57291 & 2.20598 & 0.39555 \\
\hline & $\mathrm{C}$ & -0.51832 & 0.83569 & 0.08296 \\
\hline & $\mathrm{C}$ & -1.75936 & 0.10425 & -0.02722 \\
\hline
\end{tabular}




\begin{tabular}{|c|c|c|c|c|}
\hline & $\mathrm{N}$ & -2.93282 & 0.62763 & -0.17621 \\
\hline & $\mathrm{C}$ & -3.91717 & -0.44672 & -0.06527 \\
\hline & $\mathrm{C}$ & -3.05392 & -1.72835 & -0.14929 \\
\hline & $\mathrm{O}$ & -1.72883 & -1.27744 & 0.11160 \\
\hline & $\mathrm{C}$ & -4.93340 & -0.35992 & -1.20511 \\
\hline & $\mathrm{C}$ & -4.62756 & -0.33627 & 1.29257 \\
\hline & $\mathrm{H}$ & 2.69830 & -2.58813 & 0.11450 \\
\hline & $\mathrm{H}$ & 4.03284 & 1.50295 & -0.13154 \\
\hline & $\mathrm{H}$ & 0.36155 & -1.81729 & 0.12395 \\
\hline & $\mathrm{H}$ & -1.47839 & 2.62388 & 0.83160 \\
\hline & $\mathrm{H}$ & -3.08650 & -2.18305 & -1.15109 \\
\hline & $\mathrm{H}$ & -3.31577 & -2.49245 & 0.59186 \\
\hline & $\mathrm{H}$ & -5.66326 & -1.17996 & -1.15770 \\
\hline & $\mathrm{H}$ & -4.42324 & -0.40098 & -2.17362 \\
\hline & $\mathrm{H}$ & -5.47704 & 0.58969 & -1.15300 \\
\hline & $\mathrm{H}$ & -5.40759 & -1.10260 & 1.40511 \\
\hline & $\mathrm{H}$ & -5.09030 & 0.65155 & 1.39104 \\
\hline & $\mathrm{H}$ & -3.90546 & -0.45200 & 2.10867 \\
\hline & $\mathrm{Cl}$ & 5.18165 & -1.15639 & -0.00174 \\
\hline Structure & atom & $\mathbf{X}$ & $\mathbf{Y}$ & $\mathbf{Z}$ \\
\hline & $\mathrm{C}$ & -2.44188 & -1.48739 & 0.09337 \\
\hline & $\mathrm{C}$ & -3.45703 & -0.51497 & 0.03545 \\
\hline & $\mathrm{C}$ & -3.34035 & 0.82910 & 0.00120 \\
\hline & $\mathrm{C}$ & -1.97088 & 1.25382 & 0.00810 \\
\hline & $\mathrm{C}$ & -0.84291 & 0.32621 & 0.01385 \\
\hline & $\mathrm{C}$ & -1.13076 & -1.06032 & 0.07470 \\
\hline & $\mathrm{N}$ & -1.74752 & 2.60729 & 0.01437 \\
\hline & $\mathrm{C}$ & -0.51105 & 3.06000 & 0.02525 \\
\hline & $\mathrm{C}$ & 0.63424 & 2.24774 & 0.01252 \\
\hline & $\mathrm{C}$ & 0.47960 & 0.87194 & -0.00648 \\
\hline & $\mathrm{C}$ & 1.72553 & 0.08419 & -0.03204 \\
\hline & $\mathrm{N}$ & 2.85232 & 0.47110 & 0.44274 \\
\hline & $\mathrm{C}$ & 3.85115 & -0.54730 & 0.09837 \\
\hline & $\mathrm{C}$ & 2.99168 & -1.71078 & -0.45847 \\
\hline & $\mathrm{O}$ & 1.69911 & -1.13369 & -0.65980 \\
\hline & $\mathrm{C}$ & 4.63826 & -0.95421 & 1.34441 \\
\hline & $\mathrm{C}$ & 4.79169 & 0.02641 & -0.96890 \\
\hline & $\mathrm{H}$ & -2.67000 & -2.55117 & 0.15243 \\
\hline & $\mathrm{H}$ & -0.32500 & -1.78428 & 0.10263 \\
\hline & $\mathrm{H}$ & -0.39109 & 4.14703 & 0.03469 \\
\hline & $\mathrm{H}$ & 1.63081 & 2.67912 & 0.00758 \\
\hline & $\mathrm{H}$ & 2.89620 & -2.54127 & 0.25413 \\
\hline & $\mathrm{H}$ & 3.34460 & -2.10649 & -1.41670 \\
\hline & $\mathrm{H}$ & 5.37030 & -1.73895 & 1.11231 \\
\hline & $\mathrm{H}$ & 3.96160 & -1.32733 & 2.12062 \\
\hline & $\mathrm{H}$ & 5.17482 & -0.09069 & 1.75168 \\
\hline & $\mathrm{H}$ & 5.57492 & -0.69527 & -1.23635 \\
\hline & $\mathrm{H}$ & 5.26964 & 0.93881 & -0.59690 \\
\hline
\end{tabular}




\begin{tabular}{|c|c|c|c|c|}
\hline & $\begin{array}{l}\mathrm{H} \\
\mathrm{Cl}\end{array}$ & $\begin{array}{r}4.23226 \\
-5.17054\end{array}$ & $\begin{array}{c}0.28335 \\
-1.30816\end{array}$ & $\begin{array}{r}-1.87532 \\
0.01294\end{array}$ \\
\hline \multirow[t]{31}{*}{ Structure } & atom & $\mathbf{X}$ & Y & $\mathbf{Z}$ \\
\hline & $\mathrm{C}$ & -2.25406 & -1.55676 & 0.13515 \\
\hline & $\mathrm{C}$ & -3.36467 & -0.69850 & 0.03416 \\
\hline & $\mathrm{C}$ & -3.21381 & 0.66417 & -0.03575 \\
\hline & $\mathrm{C}$ & -1.91608 & 1.23523 & -0.00559 \\
\hline & $\mathrm{C}$ & -0.76521 & 0.38815 & 0.06921 \\
\hline & $\mathrm{C}$ & -0.98729 & -1.01081 & 0.15437 \\
\hline & $\mathrm{N}$ & -1.83015 & 2.59541 & -0.02225 \\
\hline & $\mathrm{C}$ & -0.60506 & 3.09745 & 0.08438 \\
\hline & $\mathrm{C}$ & 0.67464 & 2.43859 & 0.09159 \\
\hline & $\mathrm{C}$ & 0.51379 & 1.04821 & 0.06640 \\
\hline & $\mathrm{C}$ & 1.74559 & 0.22904 & -0.02060 \\
\hline & $\mathrm{N}$ & 2.82409 & 0.36652 & 0.65306 \\
\hline & $\mathrm{C}$ & 3.80987 & -0.57734 & 0.12053 \\
\hline & $\mathrm{C}$ & 2.97865 & -1.46504 & -0.84195 \\
\hline & $\mathrm{O}$ & 1.73951 & -0.77547 & -0.97950 \\
\hline & $\mathrm{C}$ & 4.43329 & -1.38527 & 1.25950 \\
\hline & $\mathrm{C}$ & 4.88844 & 0.21569 & -0.62863 \\
\hline & $\mathrm{H}$ & -2.40233 & -2.63147 & 0.19300 \\
\hline & $\mathrm{H}$ & -4.06346 & 1.33647 & -0.09921 \\
\hline & $\mathrm{H}$ & -0.13232 & -1.67664 & 0.22003 \\
\hline & $\mathrm{H}$ & -0.61262 & 4.19522 & 0.14613 \\
\hline & $\mathrm{H}$ & 2.78769 & -2.46663 & -0.42776 \\
\hline & $\mathrm{H}$ & 3.42881 & -1.57994 & -1.83513 \\
\hline & $\mathrm{H}$ & 5.15928 & -2.11799 & 0.88107 \\
\hline & $\mathrm{H}$ & 3.65739 & -1.92097 & 1.81787 \\
\hline & $\mathrm{H}$ & 4.94806 & -0.71670 & 1.95807 \\
\hline & $\mathrm{H}$ & 5.66597 & -0.44761 & -1.03324 \\
\hline & $\mathrm{H}$ & 5.35769 & 0.93808 & 0.04739 \\
\hline & $\mathrm{H}$ & 4.43919 & 0.77643 & -1.45535 \\
\hline & $\mathrm{Cl}$ & -4.98444 & -1.40911 & 0.00651 \\
\hline \multirow[t]{13}{*}{ Structure } & atom & $\mathbf{X}$ & $\mathbf{Y}$ & $\mathbf{Z}$ \\
\hline & $\mathrm{C}$ & -2.39959 & -1.70200 & 0.11331 \\
\hline & $\mathrm{C}$ & -3.38780 & -0.72282 & 0.05350 \\
\hline & $\mathrm{C}$ & -3.25333 & 0.64470 & 0.02918 \\
\hline & $\mathrm{C}$ & -1.93884 & 1.17304 & 0.01372 \\
\hline & $\mathrm{C}$ & -0.82825 & 0.24783 & 0.01263 \\
\hline & $\mathrm{C}$ & -1.11823 & -1.14511 & 0.08642 \\
\hline & $\mathrm{N}$ & -1.79774 & 2.52584 & 0.01130 \\
\hline & $\mathrm{C}$ & -0.57268 & 3.01639 & 0.02278 \\
\hline & $\mathrm{C}$ & 0.58863 & 2.22680 & 0.01753 \\
\hline & $\mathrm{C}$ & 0.47066 & 0.84537 & -0.00809 \\
\hline & $\mathrm{C}$ & 1.73407 & 0.08587 & -0.04809 \\
\hline & $\mathrm{N}$ & 2.82790 & 0.44554 & 0.51570 \\
\hline
\end{tabular}




\begin{tabular}{|c|c|c|c|c|}
\hline & $\mathrm{C}$ & 3.86085 & -0.52121 & 0.11954 \\
\hline & $\mathrm{C}$ & 3.04950 & -1.63311 & -0.59428 \\
\hline & $\mathrm{O}$ & 1.75866 & -1.05381 & -0.80079 \\
\hline & $\mathrm{C}$ & 4.59305 & -1.04296 & 1.35576 \\
\hline & $\mathrm{C}$ & 4.84019 & 0.17204 & -0.83592 \\
\hline & $\mathrm{H}$ & -4.08669 & 1.34295 & 0.02909 \\
\hline & $\mathrm{H}$ & -0.25298 & -1.81029 & 0.13274 \\
\hline & $\mathrm{H}$ & -0.48489 & 4.10499 & 0.02865 \\
\hline & $\mathrm{H}$ & 1.57397 & 2.68348 & 0.02107 \\
\hline & $\mathrm{H}$ & 2.93158 & -2.53212 & 0.02507 \\
\hline & $\mathrm{H}$ & 3.45590 & -1.92460 & -1.56838 \\
\hline & $\mathrm{H}$ & 5.34857 & -1.79131 & 1.08225 \\
\hline & $\mathrm{H}$ & 3.88535 & -1.50202 & 2.05420 \\
\hline & $\mathrm{H}$ & 5.09434 & -0.21953 & 1.87580 \\
\hline & $\mathrm{H}$ & 5.64784 & -0.50755 & -1.13902 \\
\hline & $\mathrm{H}$ & 5.28451 & 1.04720 & -0.34984 \\
\hline & $\mathrm{H}$ & 4.31879 & 0.51380 & -1.73707 \\
\hline & $\mathrm{Cl}$ & -5.16624 & -1.27313 & 0.01381 \\
\hline Structure & atom & $\mathbf{X}$ & $\mathbf{Y}$ & $\mathbf{Z}$ \\
\hline & $\mathrm{C}$ & 2.04462 & -1.58380 & 0.01452 \\
\hline & $\mathrm{C}$ & 3.20793 & -0.78584 & -0.03332 \\
\hline & $\mathrm{C}$ & 3.19948 & 0.58973 & -0.07076 \\
\hline & $\mathrm{C}$ & 1.93925 & 1.23324 & -0.05439 \\
\hline & $\mathrm{C}$ & 0.76609 & 0.40331 & 0.02309 \\
\hline & $\mathrm{C}$ & 0.76004 & -1.03717 & 0.04794 \\
\hline & $\mathrm{N}$ & 1.90471 & 2.59927 & -0.12680 \\
\hline & $\mathrm{C}$ & 0.71652 & 3.17038 & -0.13455 \\
\hline & $\mathrm{C}$ & -0.50986 & 2.47725 & -0.06197 \\
\hline & $\mathrm{C}$ & -0.47164 & 1.09993 & 0.03265 \\
\hline & $\mathrm{C}$ & -1.71245 & 0.29764 & 0.13343 \\
\hline & $\mathrm{N}$ & -2.59807 & 0.15942 & -0.77701 \\
\hline & $\mathrm{C}$ & -3.64216 & -0.71529 & -0.21390 \\
\hline & $\mathrm{C}$ & -3.00359 & -1.20551 & 1.10973 \\
\hline & $\mathrm{O}$ & -1.94590 & -0.27829 & 1.34935 \\
\hline & $\mathrm{C}$ & -3.91847 & -1.86934 & -1.17570 \\
\hline & $\mathrm{C}$ & -4.90735 & 0.11674 & 0.02631 \\
\hline & $\mathrm{H}$ & 2.19730 & -2.66797 & 0.02299 \\
\hline & $\mathrm{H}$ & 4.10569 & 1.18283 & -0.12241 \\
\hline & $\mathrm{H}$ & 0.70852 & 4.26159 & -0.20124 \\
\hline & $\mathrm{H}$ & -1.45306 & 3.01762 & -0.07765 \\
\hline & $\mathrm{H}$ & -2.55469 & -2.20155 & 1.00165 \\
\hline & $\mathrm{H}$ & -3.68166 & -1.19409 & 1.96995 \\
\hline & $\mathrm{H}$ & -4.64656 & -2.57488 & -0.75213 \\
\hline & $\mathrm{H}$ & -2.98856 & -2.40651 & -1.38749 \\
\hline & $\mathrm{H}$ & -4.31637 & -1.48982 & -2.12348 \\
\hline & $\mathrm{H}$ & -5.73117 & -0.50551 & 0.40152 \\
\hline & $\mathrm{H}$ & -5.22689 & 0.59181 & -0.90779 \\
\hline & $\mathrm{H}$ & -4.71137 & 0.90885 & 0.75838 \\
\hline & $\mathrm{Cl}$ & 4.81819 & -1.59732 & -0.05347 \\
\hline
\end{tabular}




\begin{tabular}{|c|c|c|c|c|}
\hline \multirow[t]{35}{*}{ Structure } & atom & $\mathbf{X}$ & $\mathbf{Y}$ & $\mathbf{Z}$ \\
\hline & $\mathrm{C}$ & 0.22445 & 3.22442 & -0.22491 \\
\hline & $\mathrm{C}$ & -1.15144 & 2.91050 & -0.22518 \\
\hline & $\mathrm{C}$ & -1.58498 & 1.61024 & -0.13972 \\
\hline & $\mathrm{C}$ & -0.63910 & 0.56525 & -0.05027 \\
\hline & $\mathrm{C}$ & 0.76588 & 0.85799 & -0.03677 \\
\hline & $\mathrm{C}$ & 1.15561 & 2.22070 & -0.13043 \\
\hline & $\mathrm{N}$ & -1.10725 & -0.72262 & 0.00274 \\
\hline & $\mathrm{C}$ & -0.23398 & -1.72112 & 0.05576 \\
\hline & $\mathrm{C}$ & 1.14786 & -1.52523 & 0.08470 \\
\hline & $\mathrm{C}$ & 1.66968 & -0.24434 & 0.04722 \\
\hline & $\mathrm{C}$ & 3.14837 & -0.14878 & 0.08249 \\
\hline & $\mathrm{N}$ & 3.90911 & -1.15208 & -0.11410 \\
\hline & $\mathrm{C}$ & 5.29845 & -0.69266 & 0.03899 \\
\hline & $\mathrm{C}$ & 5.14515 & 0.83048 & 0.31862 \\
\hline & $\mathrm{O}$ & 3.71859 & 1.05082 & 0.36428 \\
\hline & $\mathrm{C}$ & 6.06657 & -0.96720 & -1.25533 \\
\hline & $\mathrm{C}$ & 5.93451 & -1.43409 & 1.21827 \\
\hline & $\mathrm{H}$ & 0.53630 & 4.26102 & -0.29844 \\
\hline & $\mathrm{H}$ & -2.64467 & 1.37636 & -0.11639 \\
\hline & $\mathrm{H}$ & 2.20851 & 2.46708 & -0.12545 \\
\hline & $\mathrm{H}$ & -0.65355 & -2.72401 & 0.06193 \\
\hline & $\mathrm{H}$ & 1.81894 & -2.37456 & 0.13391 \\
\hline & $\mathrm{H}$ & 5.55703 & 1.45996 & -0.47680 \\
\hline & $\mathrm{H}$ & 5.56202 & 1.14445 & 1.27994 \\
\hline & $\mathrm{H}$ & 7.10239 & -0.61807 & -1.17044 \\
\hline & $\mathrm{H}$ & 5.59748 & -0.45746 & -2.10363 \\
\hline & $\mathrm{H}$ & 6.08001 & -2.04035 & -1.46839 \\
\hline & $\mathrm{H}$ & 6.97353 & -1.11377 & 1.35940 \\
\hline & $\mathrm{H}$ & 5.92707 & -2.51299 & 1.03598 \\
\hline & $\mathrm{H}$ & 5.38401 & -1.24063 & 2.14528 \\
\hline & $\mathrm{Cl}$ & -2.30212 & 4.20256 & -0.34007 \\
\hline & $\mathrm{Zn}$ & -3.03867 & -1.32254 & 0.04719 \\
\hline & $\mathrm{Cl}$ & -4.53452 & -0.05028 & 0.92470 \\
\hline & $\mathrm{Cl}$ & -3.12413 & -3.33767 & -0.73216 \\
\hline \multirow[t]{14}{*}{ Structure } & atom & $\mathbf{X}$ & $\mathbf{Y}$ & $\mathbf{Z}$ \\
\hline & $\mathrm{C}$ & -2.72172 & 3.04410 & -0.00183 \\
\hline & $\mathrm{C}$ & -1.56483 & 3.84936 & -0.02341 \\
\hline & $\mathrm{C}$ & -0.31000 & 3.29850 & -0.03167 \\
\hline & $\mathrm{C}$ & -0.15448 & 1.88690 & -0.01899 \\
\hline & $\mathrm{C}$ & -1.31699 & 1.05307 & -0.01087 \\
\hline & $\mathrm{C}$ & -2.59191 & 1.67398 & 0.00412 \\
\hline & $\mathrm{N}$ & 1.12443 & 1.42024 & -0.00818 \\
\hline & $\mathrm{C}$ & 1.35087 & 0.10613 & 0.02474 \\
\hline & $\mathrm{C}$ & 0.23852 & -0.78866 & 0.03681 \\
\hline & $\mathrm{C}$ & -1.07111 & -0.35642 & 0.01092 \\
\hline & $\mathrm{C}$ & -2.12347 & -1.39030 & 0.00913 \\
\hline & $\mathrm{N}$ & -1.99155 & -2.56834 & 0.48362 \\
\hline & $\mathrm{C}$ & -3.21798 & -3.31318 & 0.17484 \\
\hline
\end{tabular}




\begin{tabular}{|c|c|c|c|c|}
\hline & $\mathrm{C}$ & -4.15501 & -2.22951 & -0.42534 \\
\hline & $\mathrm{O}$ & -3.31424 & -1.08028 & -0.59620 \\
\hline & $\mathrm{C}$ & -3.78815 & -3.92494 & 1.45510 \\
\hline & $\mathrm{C}$ & -2.88213 & -4.40657 & -0.84544 \\
\hline & $\mathrm{H}$ & -3.70251 & 3.50929 & 0.00941 \\
\hline & $\mathrm{H}$ & 0.59052 & 3.90278 & -0.04076 \\
\hline & $\mathrm{H}$ & -3.48207 & 1.05656 & 0.01210 \\
\hline & $\mathrm{H}$ & 0.41972 & -1.85985 & 0.05234 \\
\hline & $\mathrm{H}$ & -4.97839 & -1.96003 & 0.24856 \\
\hline & $\mathrm{H}$ & -4.56944 & -2.49768 & -1.40256 \\
\hline & $\mathrm{H}$ & -4.71908 & -4.46950 & 1.25114 \\
\hline & $\mathrm{H}$ & -3.99602 & -3.14520 & 2.19589 \\
\hline & $\mathrm{H}$ & -3.06734 & -4.62255 & 1.89333 \\
\hline & $\mathrm{H}$ & -3.77192 & -5.00001 & -1.09291 \\
\hline & $\mathrm{H}$ & -2.11656 & -5.07643 & -0.44131 \\
\hline & $\mathrm{H}$ & -2.49038 & -3.96522 & -1.76834 \\
\hline & $\mathrm{Cl}$ & -1.76043 & 5.59564 & -0.03496 \\
\hline & $\mathrm{Zn}$ & 3.22745 & -0.49324 & 0.00510 \\
\hline & $\mathrm{Cl}$ & 4.15605 & -1.23296 & -1.88753 \\
\hline & $\mathrm{Cl}$ & 4.51579 & -0.56330 & 1.81796 \\
\hline Structure & atom & $\mathbf{X}$ & $\mathbf{Y}$ & $\mathbf{Z}$ \\
\hline & $\mathrm{C}$ & 0.12554 & 2.59018 & 0.03306 \\
\hline & $\mathrm{C}$ & 1.40725 & 1.99425 & 0.02490 \\
\hline & $\mathrm{C}$ & 1.61991 & 0.64263 & 0.00678 \\
\hline & $\mathrm{C}$ & 0.43979 & -0.15510 & -0.00707 \\
\hline & $\mathrm{C}$ & -0.88973 & 0.39141 & -0.01463 \\
\hline & $\mathrm{C}$ & -1.00360 & 1.80519 & 0.01244 \\
\hline & $\mathrm{N}$ & 0.67984 & -1.49825 & -0.00610 \\
\hline & $\mathrm{C}$ & -0.34409 & -2.32244 & -0.00225 \\
\hline & $\mathrm{C}$ & -1.68388 & -1.89506 & -0.00988 \\
\hline & $\mathrm{C}$ & -1.97348 & -0.54150 & -0.02337 \\
\hline & $\mathrm{C}$ & -3.40566 & -0.18203 & -0.03095 \\
\hline & $\mathrm{N}$ & -4.34995 & -0.95844 & 0.34486 \\
\hline & $\mathrm{C}$ & -5.62029 & -0.26705 & 0.09105 \\
\hline & $\mathrm{C}$ & -5.17188 & 1.15768 & -0.33168 \\
\hline & $\mathrm{O}$ & -3.75334 & 1.04902 & -0.51083 \\
\hline & $\mathrm{C}$ & -6.46758 & -0.25134 & 1.36376 \\
\hline & $\mathrm{C}$ & -6.35710 & -0.99294 & -1.04060 \\
\hline $\mathrm{ZnCl}_{2}$ & $\mathrm{H}$ & 0.03493 & 3.67303 & 0.05505 \\
\hline & $\mathrm{H}$ & -1.98128 & 2.26955 & 0.01141 \\
\hline & $\mathrm{H}$ & -0.11260 & -3.38693 & 0.00179 \\
\hline & $\mathrm{H}$ & -2.49615 & -2.61424 & -0.00894 \\
\hline & $\mathrm{H}$ & -5.36487 & 1.90993 & 0.44357 \\
\hline & $\mathrm{H}$ & -5.60968 & 1.49832 & -1.27516 \\
\hline & $\mathrm{H}$ & -7.40964 & 0.28801 & 1.20243 \\
\hline & $\mathrm{H}$ & -5.92462 & 0.23325 & 2.18219 \\
\hline & $\mathrm{H}$ & -6.70359 & -1.27440 & 1.67405 \\
\hline & $\mathrm{H}$ & -7.32274 & -0.51522 & -1.25052 \\
\hline & $\mathrm{H}$ & -6.53749 & -2.03679 & -0.76405 \\
\hline
\end{tabular}




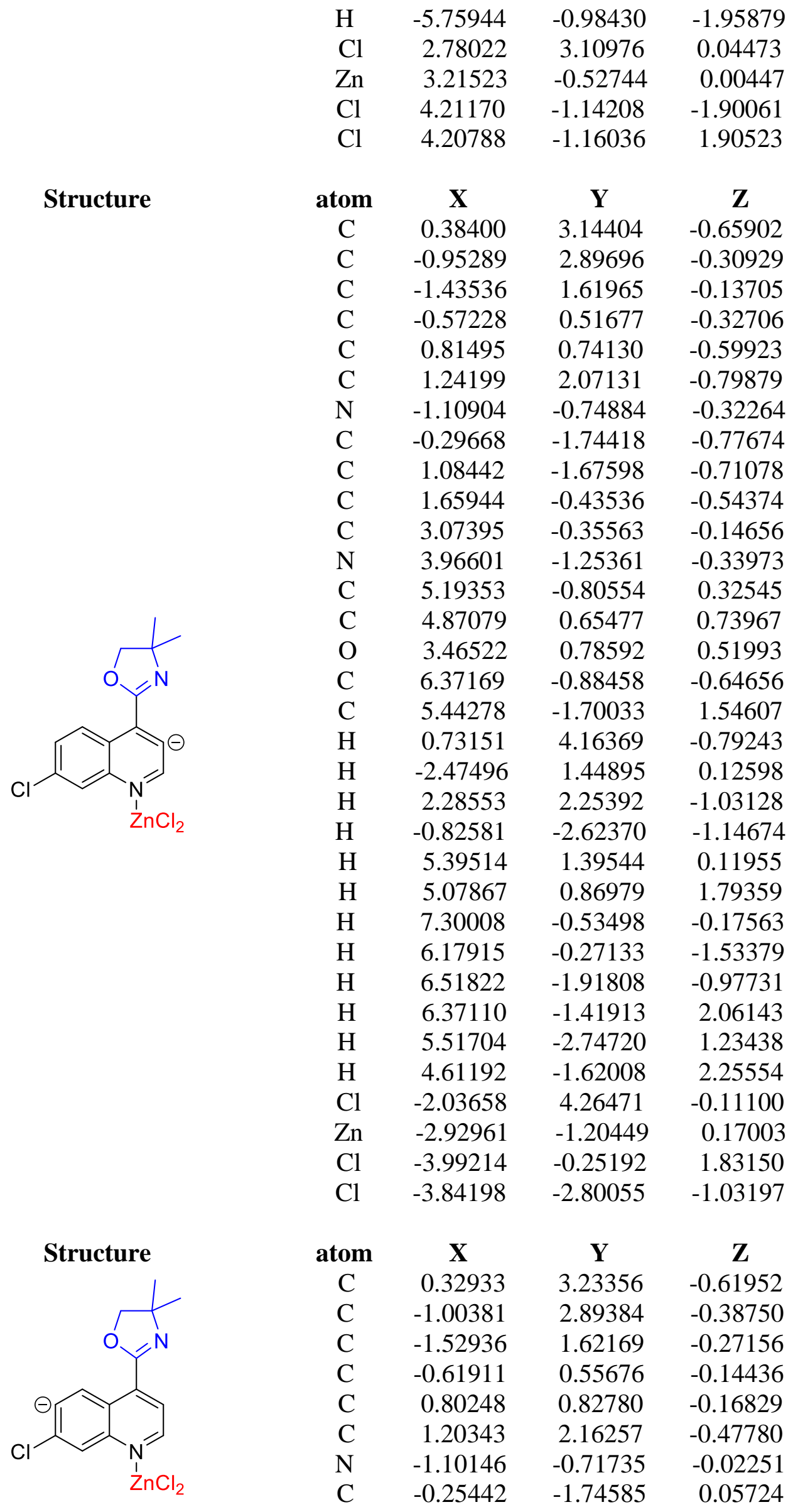




\begin{tabular}{|c|c|c|c|c|}
\hline & $\mathrm{C}$ & 1.12362 & -1.57697 & 0.07153 \\
\hline & $\mathrm{C}$ & 1.66043 & -0.29309 & -0.00309 \\
\hline & $\mathrm{C}$ & 3.13207 & -0.20406 & 0.07973 \\
\hline & $\mathrm{N}$ & 3.93219 & -1.15017 & -0.24219 \\
\hline & $\mathrm{C}$ & 5.29202 & -0.70297 & 0.09167 \\
\hline & $\mathrm{C}$ & 5.08985 & 0.78516 & 0.48468 \\
\hline & $\mathrm{O}$ & 3.66833 & 0.93863 & 0.58869 \\
\hline & $\mathrm{C}$ & 6.20633 & -0.86161 & -1.12313 \\
\hline & $\mathrm{C}$ & 5.80524 & -1.54007 & 1.26915 \\
\hline & $\mathrm{H}$ & -2.59903 & 1.42130 & -0.22638 \\
\hline & $\mathrm{H}$ & 2.26706 & 2.31121 & -0.66398 \\
\hline & $\mathrm{H}$ & -0.70085 & -2.73579 & 0.09921 \\
\hline & $\mathrm{H}$ & 1.78367 & -2.43368 & 0.14521 \\
\hline & $\mathrm{H}$ & 5.45495 & 1.47988 & -0.28197 \\
\hline & $\mathrm{H}$ & 5.53328 & 1.05389 & 1.44853 \\
\hline & $\mathrm{H}$ & 7.22056 & -0.50567 & -0.90119 \\
\hline & $\mathrm{H}$ & 5.81722 & -0.29354 & -1.97475 \\
\hline & $\mathrm{H}$ & 6.26616 & -1.91447 & -1.41796 \\
\hline & $\mathrm{H}$ & 6.82930 & -1.25214 & 1.53973 \\
\hline & $\mathrm{H}$ & 5.79988 & -2.60317 & 1.00693 \\
\hline & $\mathrm{H}$ & 5.16380 & -1.40390 & 2.14691 \\
\hline & $\mathrm{Cl}$ & -2.21680 & 4.22717 & -0.22209 \\
\hline & $\mathrm{Zn}$ & -3.01156 & -1.17491 & 0.06280 \\
\hline & $\mathrm{Cl}$ & -4.41645 & -0.30323 & 1.46764 \\
\hline & $\mathrm{Cl}$ & -3.43026 & -2.93884 & -1.17576 \\
\hline Structure & atom & $\mathbf{X}$ & $\mathbf{Y}$ & $\mathbf{Z}$ \\
\hline & $\mathrm{C}$ & 0.91310 & 2.84383 & -0.19404 \\
\hline & $\mathrm{C}$ & -0.49515 & 2.81317 & -0.24034 \\
\hline & $\mathrm{C}$ & -1.24379 & 1.65427 & -0.16175 \\
\hline & $\mathrm{C}$ & -0.53892 & 0.44795 & -0.02253 \\
\hline & $\mathrm{C}$ & 0.89989 & 0.49466 & 0.04254 \\
\hline & $\mathrm{C}$ & 1.69073 & 1.69407 & -0.04797 \\
\hline & $\mathrm{N}$ & -1.22589 & -0.74683 & 0.02613 \\
\hline & $\mathrm{C}$ & -0.54045 & -1.88759 & 0.11565 \\
\hline & $\mathrm{C}$ & 0.85108 & -1.93994 & 0.19712 \\
\hline & $\mathrm{C}$ & 1.55909 & -0.74866 & 0.17550 \\
\hline & $\mathrm{C}$ & 3.03961 & -0.72513 & 0.26708 \\
\hline & $\mathrm{N}$ & 3.84625 & -1.03777 & -0.66713 \\
\hline & $\mathrm{C}$ & 5.18274 & -0.63571 & -0.18513 \\
\hline & $\mathrm{C}$ & 4.96307 & -0.45656 & 1.33654 \\
\hline $\mathrm{ZnCl}_{2}$ & $\mathrm{O}$ & 3.54090 & -0.35305 & 1.47718 \\
\hline & $\mathrm{C}$ & 5.50142 & 0.70929 & -0.85074 \\
\hline & $\mathrm{C}$ & 6.22739 & -1.69720 & -0.51309 \\
\hline & $\mathrm{H}$ & -2.32838 & 1.67347 & -0.18852 \\
\hline & $\mathrm{H}$ & -1.13341 & -2.79902 & 0.10817 \\
\hline & $\mathrm{H}$ & 1.35324 & -2.89880 & 0.27107 \\
\hline & $\mathrm{H}$ & 5.41498 & 0.45329 & 1.74226 \\
\hline & $\mathrm{H}$ & 5.30812 & -1.32339 & 1.91881 \\
\hline & $\mathrm{H}$ & 6.46004 & 1.11032 & -0.49389 \\
\hline
\end{tabular}




\begin{tabular}{lrrr}
$\mathrm{H}$ & 4.69474 & 1.41870 & -0.62666 \\
$\mathrm{H}$ & 5.55546 & 0.58766 & -1.93800 \\
$\mathrm{H}$ & 7.22043 & -1.40008 & -0.15179 \\
$\mathrm{H}$ & 6.28664 & -1.84429 & -1.59665 \\
$\mathrm{H}$ & 5.96512 & -2.65802 & -0.05502 \\
$\mathrm{Cl}$ & -1.38152 & 4.34260 & -0.42403 \\
$\mathrm{Zn}$ & -3.17977 & -0.94334 & 0.01122 \\
$\mathrm{Cl}$ & -4.58391 & 0.39161 & 0.99255 \\
$\mathrm{Cl}$ & -3.77060 & -2.84775 & -0.91479 \\
$\mathrm{H}$ & 1.37988 & 3.82842 & -0.28308 \\
\hline
\end{tabular}

6. Flow configurations: details regarding flow-rate, residence time, coil reactors and work-up procedures

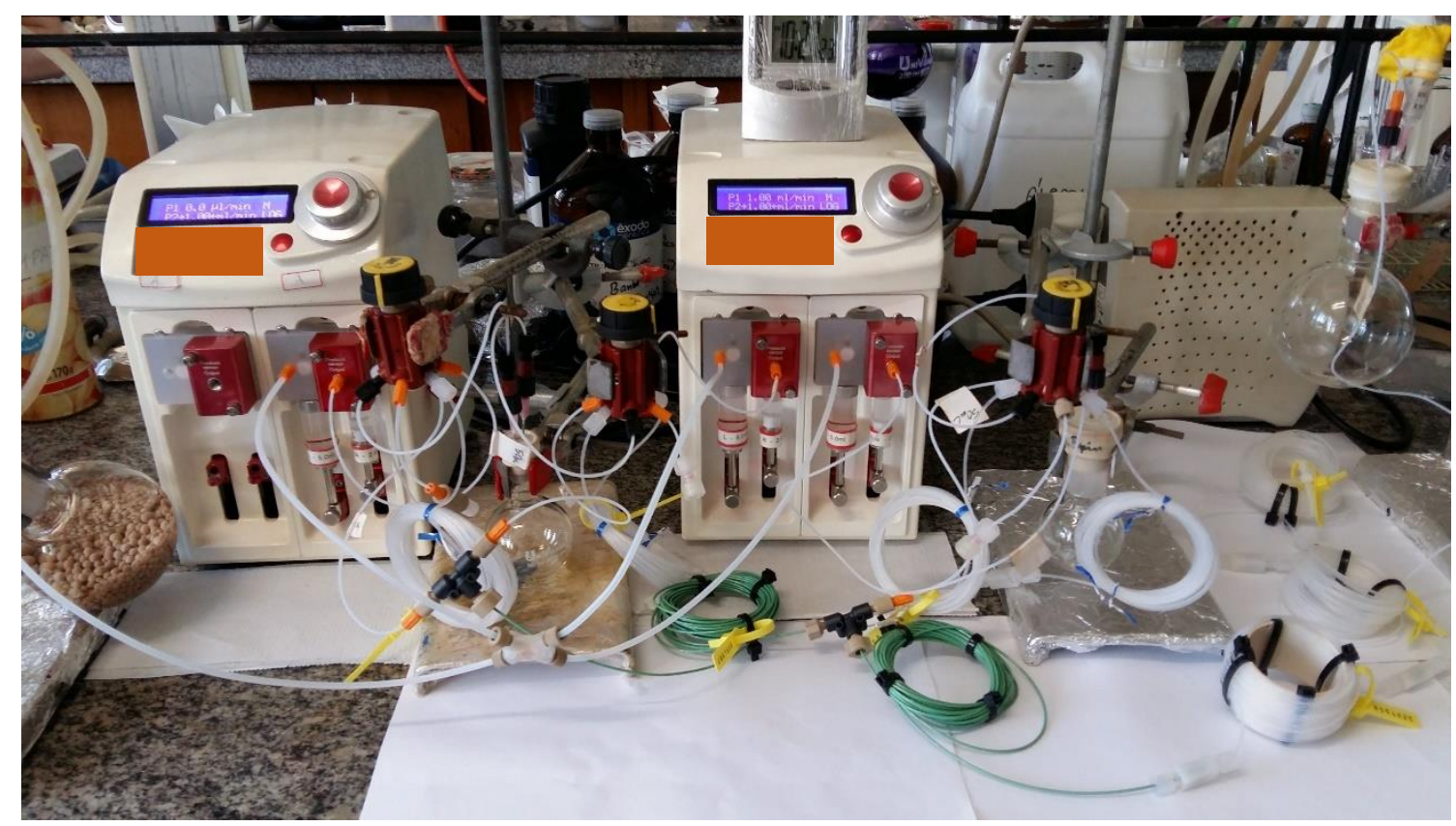

Figure S7. Flow apparatus using manual injection valves and Syrris Asia Flow Chemistry Syringe Pump ${ }^{\circledR}$. 


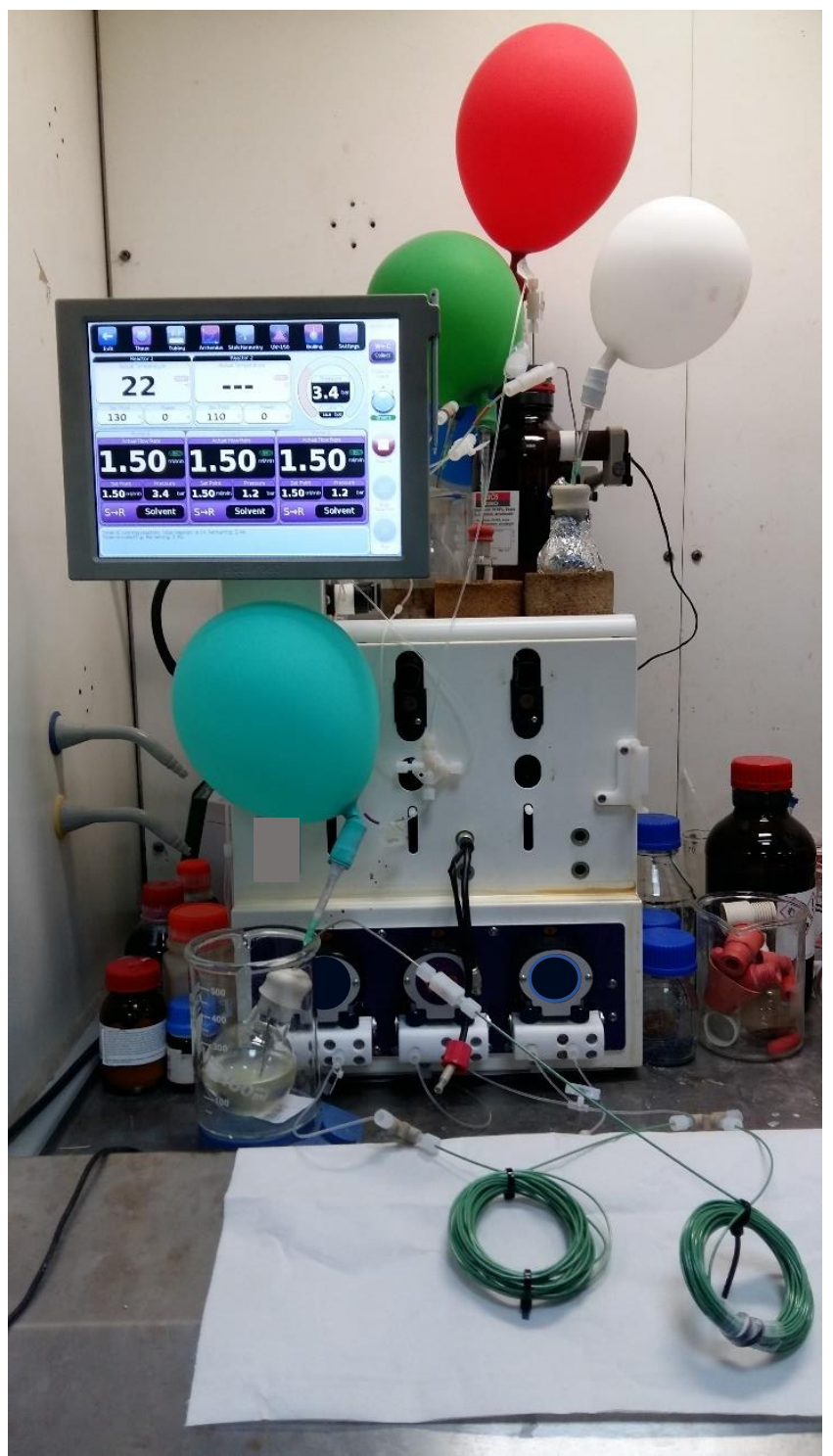

Figure S8. Flow apparatus using Vapourtec E-series Integrated Flow Chemistry System ${ }^{\circledR}$. 
6.1. Halogen-metal exchange reaction followed by the reaction with benzaldehyde using the Syrris Asia Flow Chemistry System

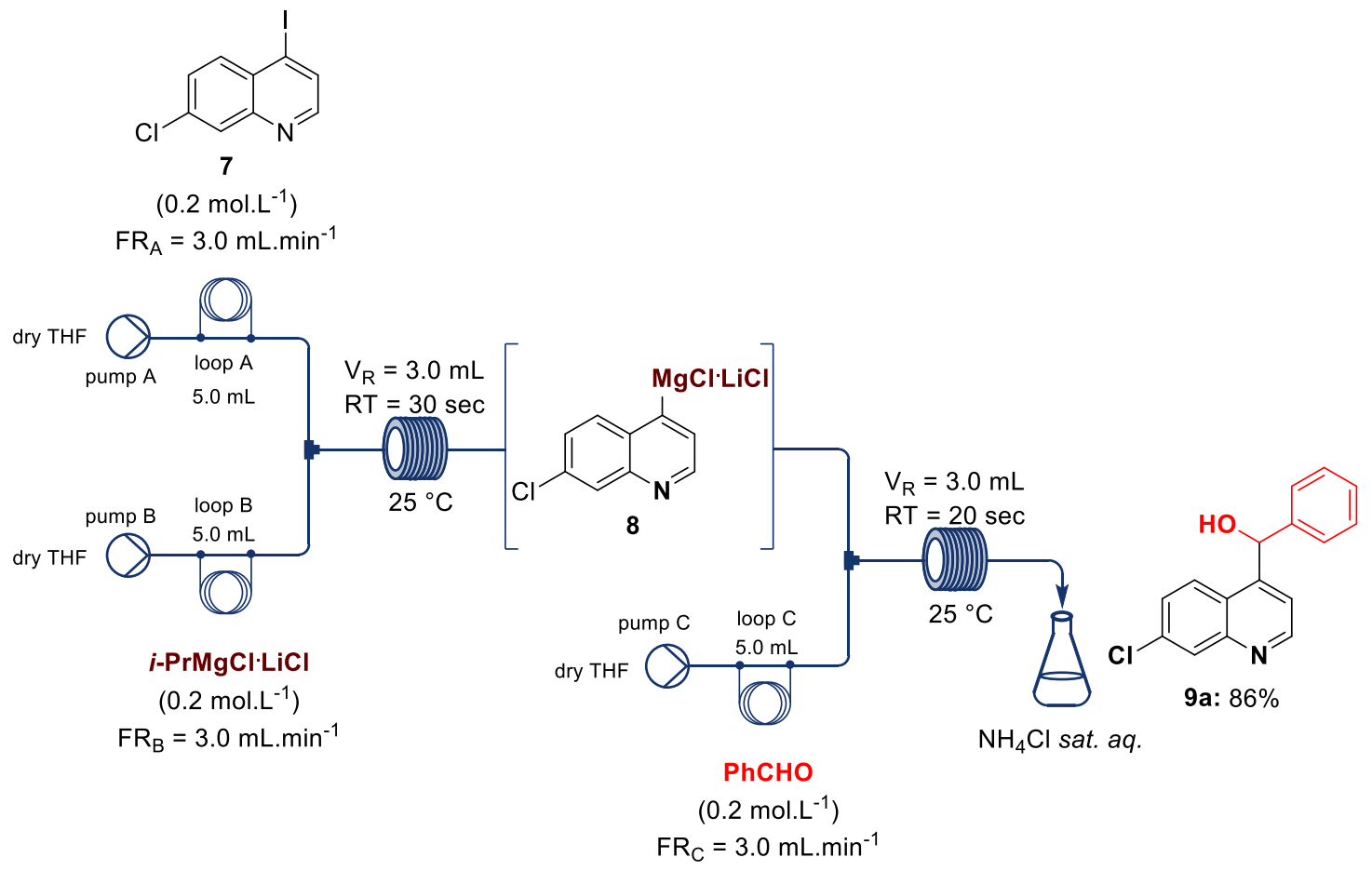

Scheme S3. Flow parameters for iodo-magnesium exchange using benzaldehyde as an electrophile.

6.2. Halogen-metal exchange reaction followed by the reaction with diphenyl diselenide or $p$-toluenesulfonyl cyanide using the Syrris Asia Flow Chemistry System

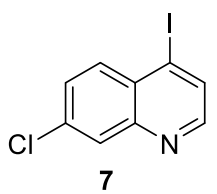

$\left(0.2 \mathrm{~mol}^{\left.-\mathrm{L}^{-1}\right)}\right.$

$\mathrm{FR}_{\mathrm{A}}=3.0 \mathrm{~mL} \cdot \mathrm{min}^{-1}$

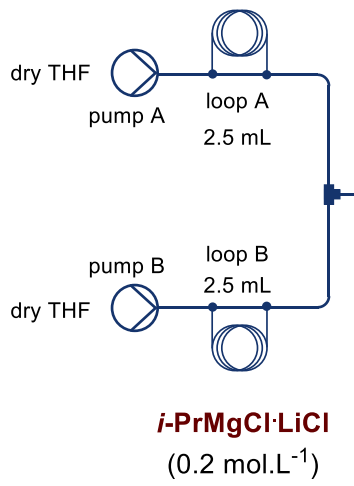

$\mathrm{FR}_{\mathrm{B}}=3.0 \mathrm{~mL} \cdot \mathrm{min}^{-1}$

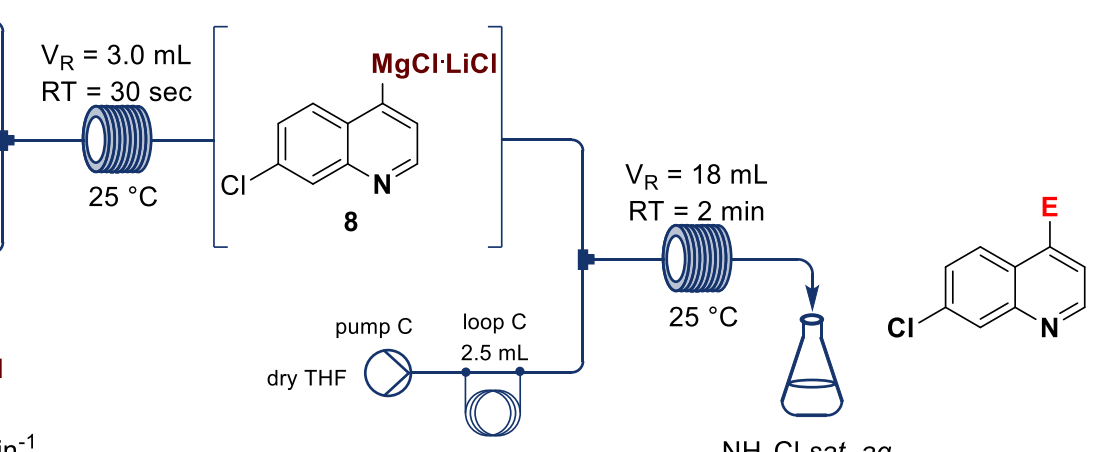

$\mathrm{NH}_{4} \mathrm{Cl}$ sat. aq.

Electrophile

( 0.3 or $\left.0.4 \mathrm{~mol}^{-\mathrm{L}^{-1}}\right)$

$\mathrm{FR}_{\mathrm{C}}=3.0 \mathrm{~mL} \cdot \mathrm{min}^{-1}$

Scheme S4. Flow parameters for iodo-magnesium exchange using different electrophiles. 
6.3. Halogen-metal exchange reaction followed by the reaction with cyclohexanone or DMF using the Syrris Asia Flow Chemistry System

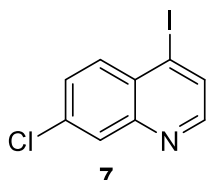

(0.2 mol. $\left.\mathrm{L}^{-1}\right)$

$\mathrm{FR}_{\mathrm{A}}=1.2 \mathrm{~mL} \cdot \mathrm{min}^{-1}$

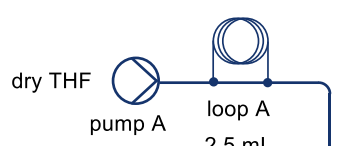

$\mathrm{V}_{\mathrm{R}}=3.0 \mathrm{~mL}$

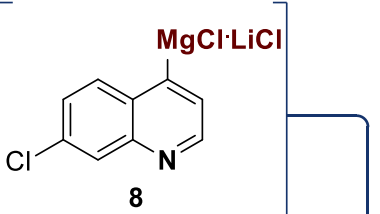

dry THF

pump B loop B

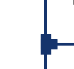

$=1.25 \mathrm{~min}$

$2.5 \mathrm{~mL}$

$25^{\circ} \mathrm{C}$

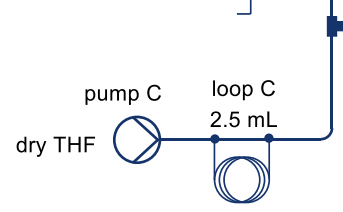

$\mathrm{V}_{\mathrm{R}}=18 \mathrm{~mL}$

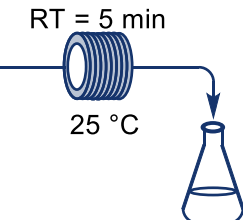<smiles>Fc1ccnc2cc(Cl)ccc12</smiles>

i-PrMgCl'LiC

$\left(0.2 \mathrm{~mol}^{\left.-\mathrm{L}^{-1}\right)}\right.$

$\mathrm{FR}_{\mathrm{B}}=1.2 \mathrm{~mL} \cdot \mathrm{min}^{-1}$

Electrophile

$\left(1.2 \mathrm{~mol} . \mathrm{L}^{-1}\right)$

$\mathrm{FR}_{\mathrm{C}}=1.2 \mathrm{~mL} \cdot \mathrm{min}^{-1}$

Scheme S5. Flow parameters for iodo-magnesium exchange using cyclohexanone or DMF.

Table S4. Optimization of flow conditions

\begin{tabular}{ccccc}
\hline Entry & Electrophile & $\begin{array}{c}\text { Flowrate } \\
\left(\mathrm{FR}_{\mathrm{A}}=\mathrm{FR} \mathrm{B}_{\mathrm{B}}=\mathrm{FR}\right)\end{array}$ & Residence time & $\begin{array}{c}\text { Isolated yield } \\
(\%)\end{array}$ \\
\hline $1^{a}$ & cyclohexanone & $3.0 \mathrm{~mL} \cdot \mathrm{min}^{-1}$ & $20 \mathrm{sec}^{c}$ & 22 \\
$2^{a}$ & cyclohexanone & $3.0 \mathrm{~mL} \cdot \mathrm{min}^{-1}$ & $2 \mathrm{~min}^{c}$ & 37 \\
$3^{b}$ & cyclohexanone & $1.2 \mathrm{~mL} \cdot \mathrm{min}^{-1}$ & $5 \mathrm{~min}^{d}$ & 53 \\
$4^{a}$ & DMF & $3.0 \mathrm{~mL} \cdot \mathrm{min}^{-1}$ & $20 \mathrm{sec}^{c}$ & 28 \\
$5^{b}$ & DMF & $1.2 \mathrm{~mL} \cdot \mathrm{min}^{-1}$ & $5 \mathrm{~min}^{d}$ & 71
\end{tabular}

${ }^{a}$ Metalation step: $\mathrm{RT}=30 \mathrm{sec}$

${ }^{b}$ Metalation step: $\mathrm{RT}=1 \mathrm{~min} 15 \mathrm{sec}$

${ }^{c}$ Reaction between organomagnesium intermediate 7 and electrophile: Volume of coil $=$ $3 \mathrm{~mL}$

${ }^{d}$ Reaction between organomagnesium intermediate 7 and electrophile: Volume of coil $=$ $18 \mathrm{~mL}$ 
<smiles>Clc1ccc2c(Cl)ccnc2c1</smiles>

(0.2 $\left.\mathrm{mol}^{-1}\right)$

$\mathrm{FR}_{\mathrm{A}}=1.5 \mathrm{~mL} \cdot \mathrm{min}^{-1}$

TMPMgCl'LiCl

$\left(0.3 \mathrm{~mol}^{-\mathrm{L}^{-1}}\right)$

$\mathrm{FR}_{\mathrm{B}}=1.5 \mathrm{~mL} \cdot \mathrm{min}^{-1}$

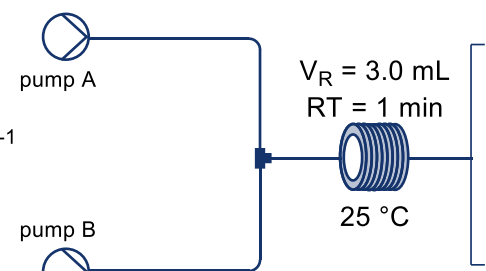

(1)

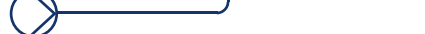

(1)

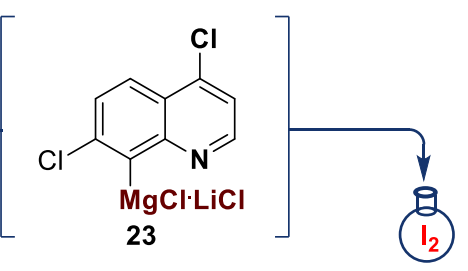

$25^{\circ} \mathrm{C}$<smiles>Cc1c(Cl)ccc2c(Cl)ccnc12</smiles>

24a: $92 \%$

Scheme S6. Flow parameters for magnesiation using mixed lithium-magnesium base and iodine as an electrophile.

6.5. Metalation reaction followed by the reaction with aldehydes using the Vapourtec E-series Integrated Flow Chemistry System

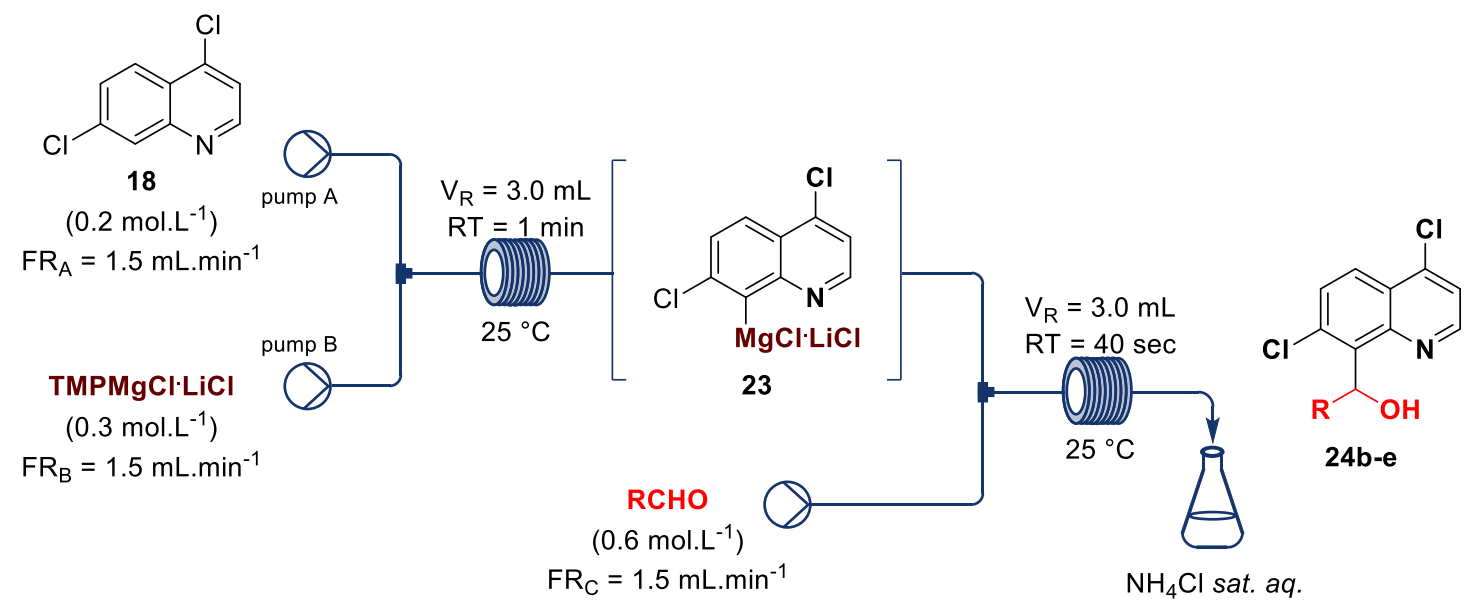

Scheme S7. Flow parameters for magnesiation using mixed lithium-magnesium base and different aldehydes. 
6.6. Metalation reaction followed by the reaction with diphenyl diselenide using the Syrris Asia Flow Chemistry System

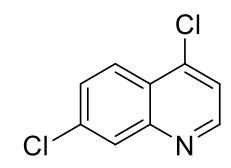

18

(0.2 mol. $\left.\mathrm{L}^{-1}\right)$

$\mathrm{FR}_{\mathrm{A}}=1.5 \mathrm{~mL} \cdot \mathrm{min}^{-1}$

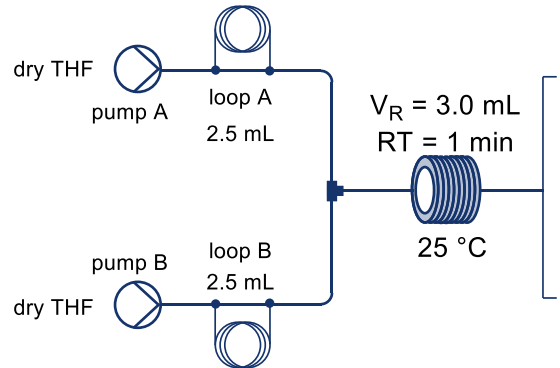

TMPMgCl·LiCl

(0.3 mol.L $\left.{ }^{-1}\right)$

$\mathrm{FR}_{\mathrm{B}}=1.5 \mathrm{~mL} \cdot \mathrm{min}^{-1}$

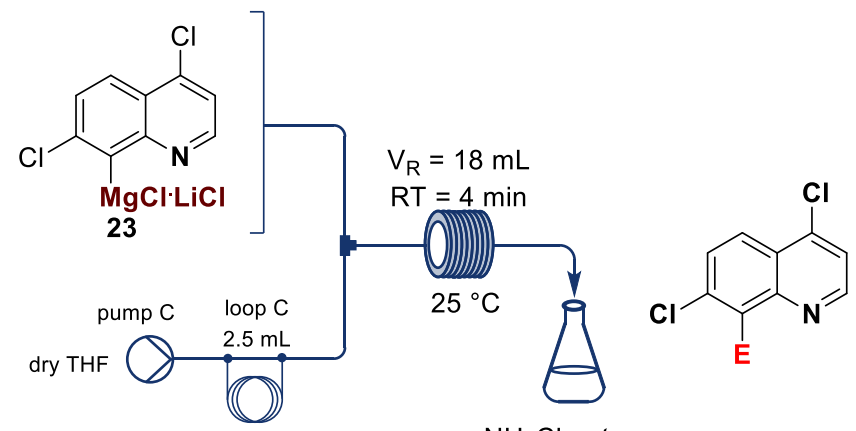

$\mathrm{NH}_{4} \mathrm{Cl}$ sat. aq.

$$
\begin{aligned}
& \text { Electrophile } \\
& \left(0.4 \text { mol. } \mathrm{L}^{-1}\right)
\end{aligned}
$$$$
\mathrm{FR}_{\mathrm{C}}=1.5 \mathrm{~mL} \cdot \mathrm{min}^{-1}
$$

Scheme S8. Flow parameters for magnesiation using mixed lithium-magnesium base and diphenyl diselenide as an electrophile. 
6.7. Metalation reaction followed by the reaction with DMF or 1,2dibromotetrachloroethane using the Syrris Asia Flow Chemistry System

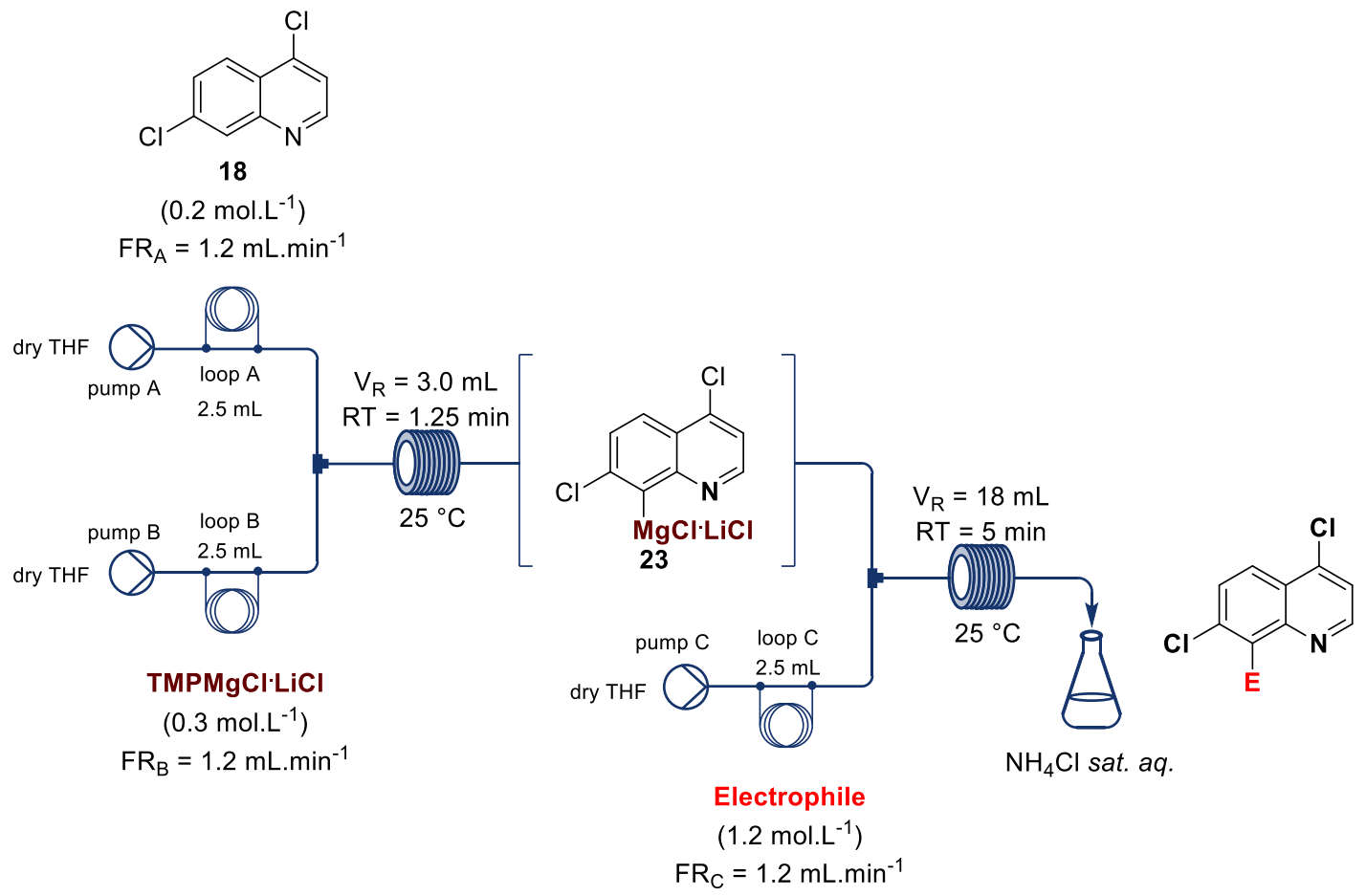

Scheme S9. Flow parameters for magnesiation using mixed lithium-magnesium base and different electrophiles.

\section{References}

(1) Mosmann, T. Rapid Colorimetric Assay for Cellular Growth and Survival: Application to Proliferation and Cytotoxicity Assays. J. Immunol. Methods 1983, 65, 55-63. https://doi.org/10.1016/0022-1759(83)90303-4. 
8. ${ }^{1} \mathrm{H}$ NMR and ${ }^{13} \mathrm{C}$ NMR spectra for all compounds ${ }^{1} \mathrm{H}$ NMR spectrum in $\mathrm{CDCl}_{3}$ of compound 9a

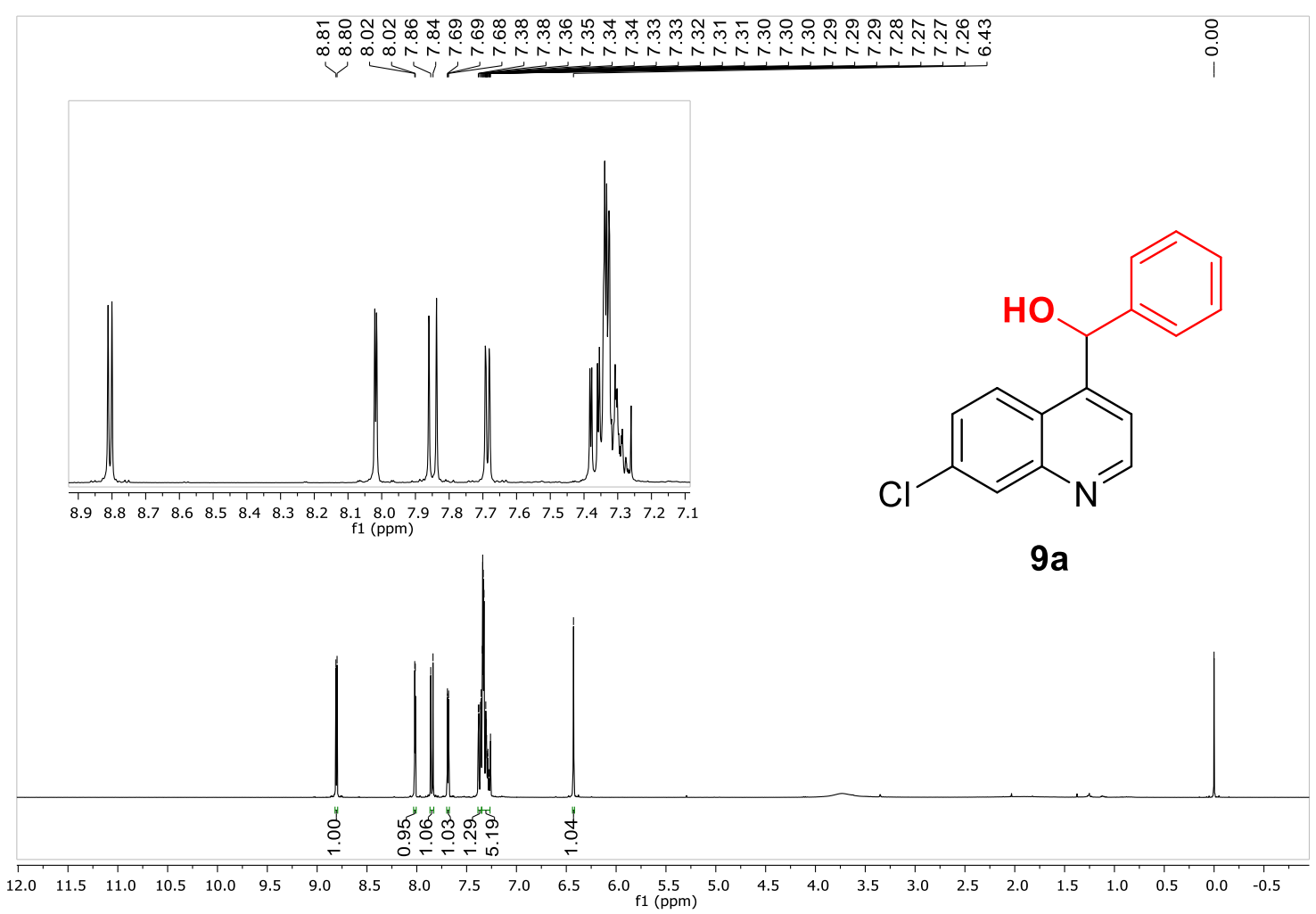

${ }^{13} \mathrm{C}\left\{{ }^{1} \mathrm{H}\right\}$ NMR spectrum in $\mathrm{CDCl}_{3}$ of compound $9 \mathbf{a}$

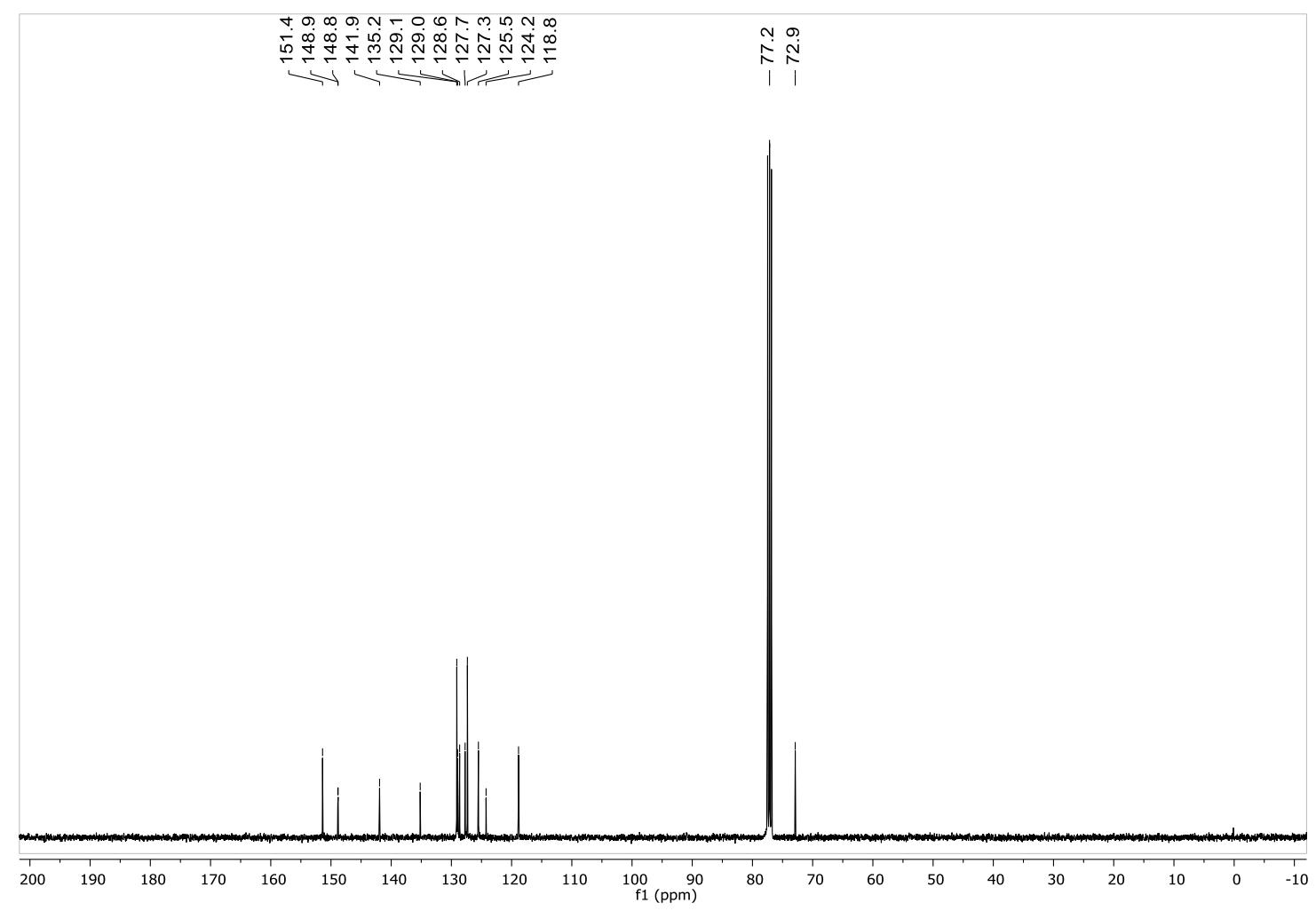


${ }^{1} \mathrm{H}$ NMR spectrum in $\mathrm{CDCl}_{3}$ of compound $\mathbf{9 b}$

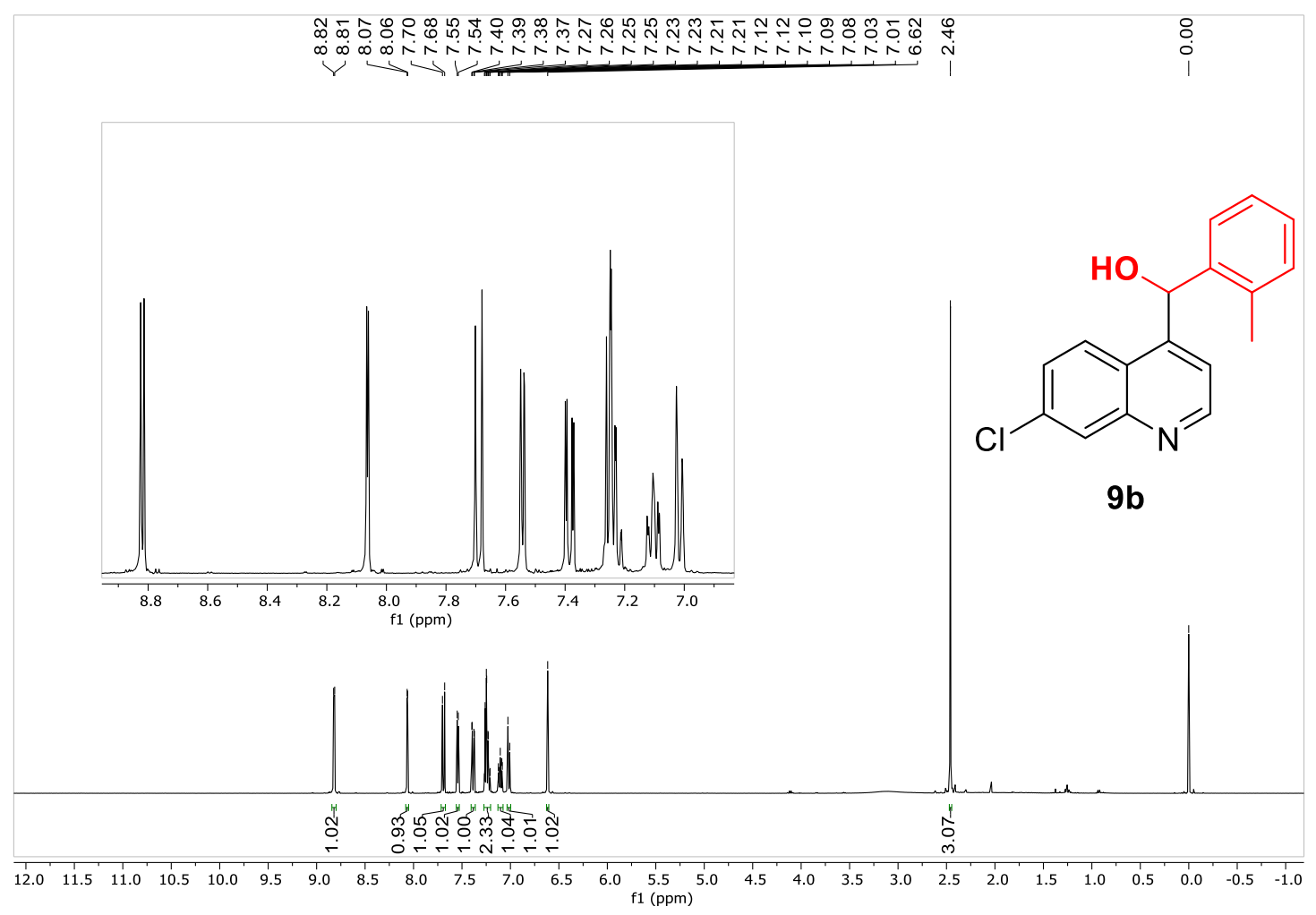

${ }^{13} \mathrm{C}\left\{{ }^{1} \mathrm{H}\right\}$ NMR spectrum in $\mathrm{CDCl}_{3}$ of compound $\mathbf{9 b}$

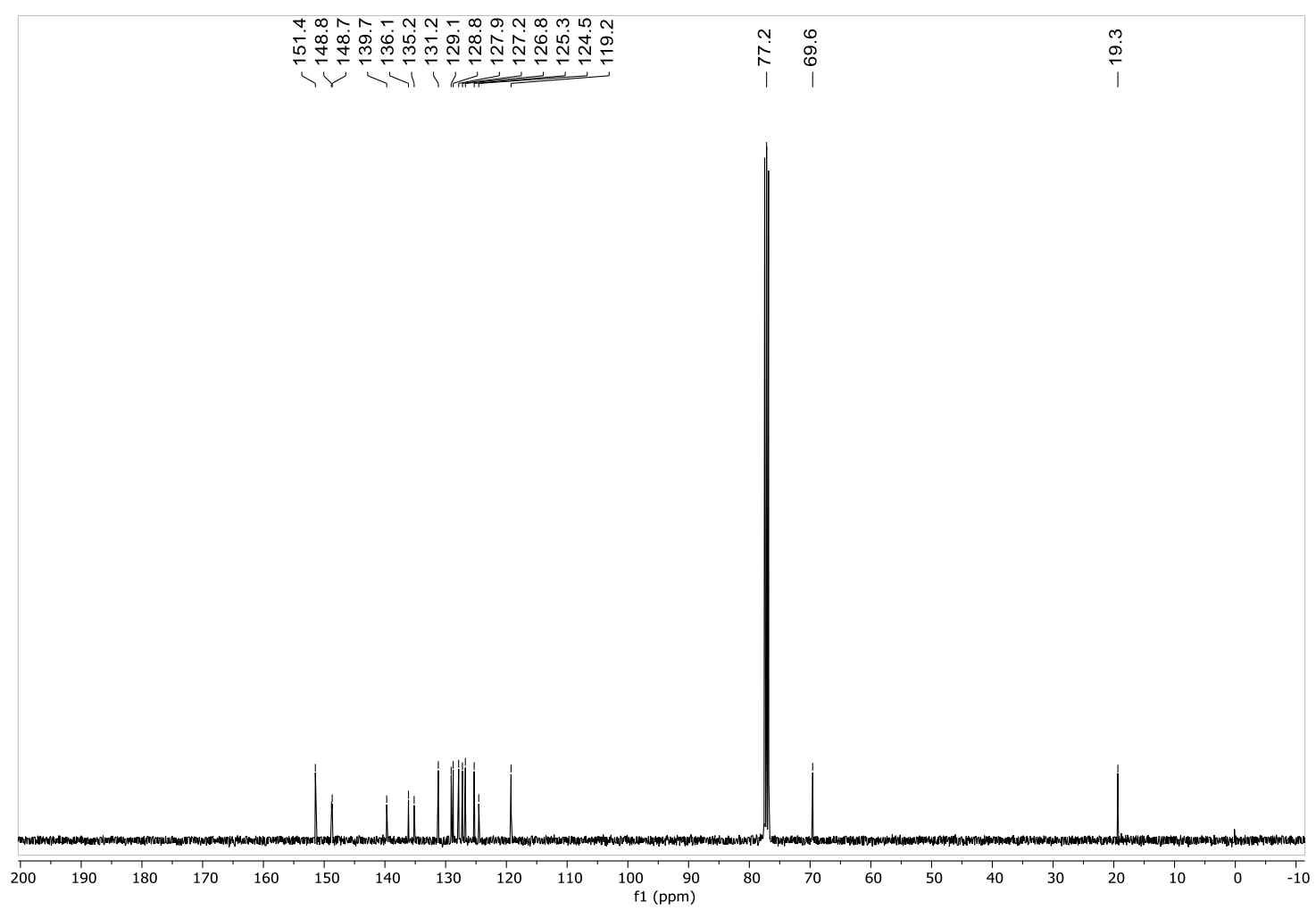


${ }^{1} \mathrm{H}$ NMR spectrum in $\mathrm{CDCl}_{3}$ of compound $9 \mathrm{c}$

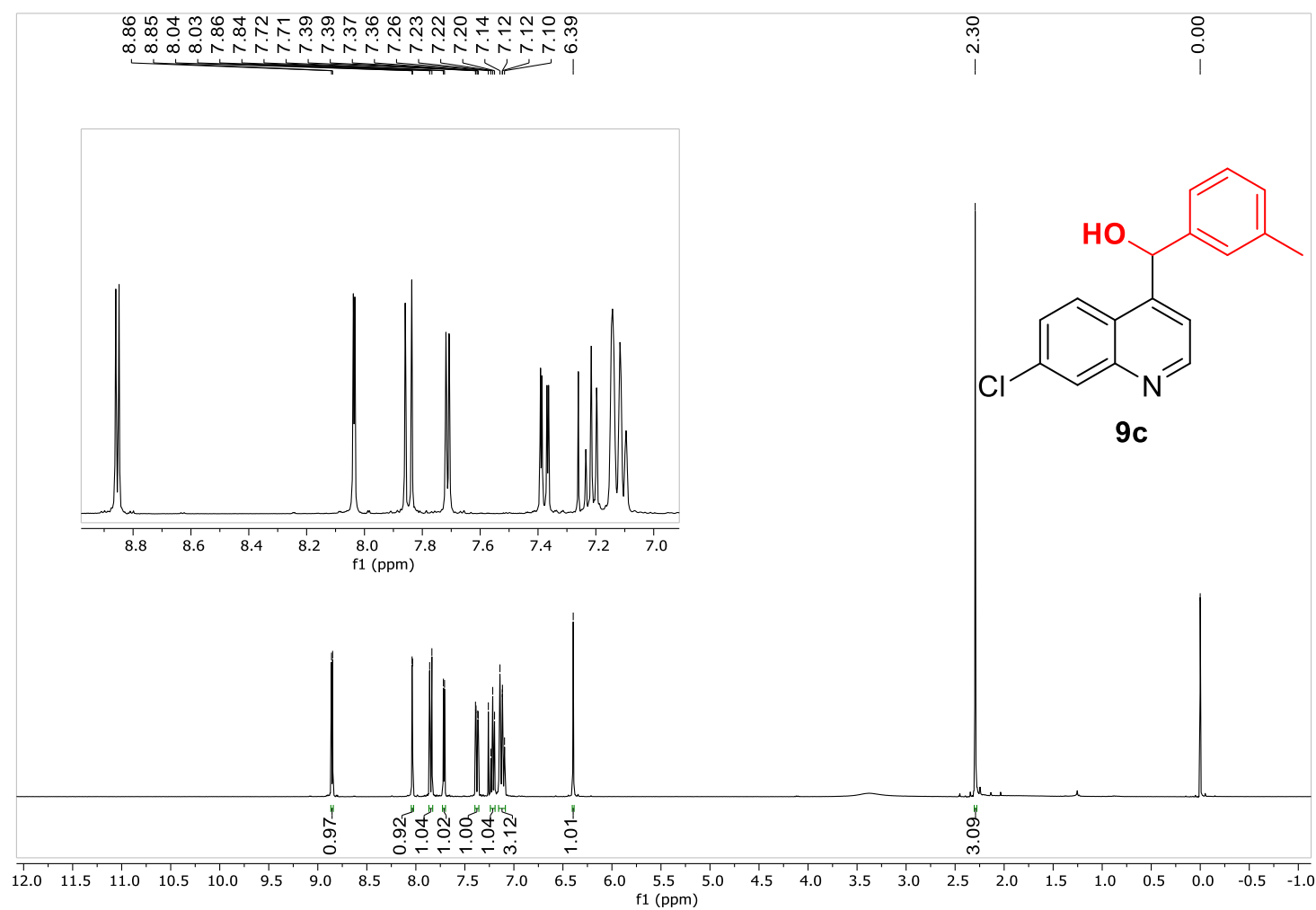

${ }^{13} \mathrm{C}\left\{{ }^{1} \mathrm{H}\right\}$ NMR spectrum in $\mathrm{CDCl}_{3}$ of compound 9c

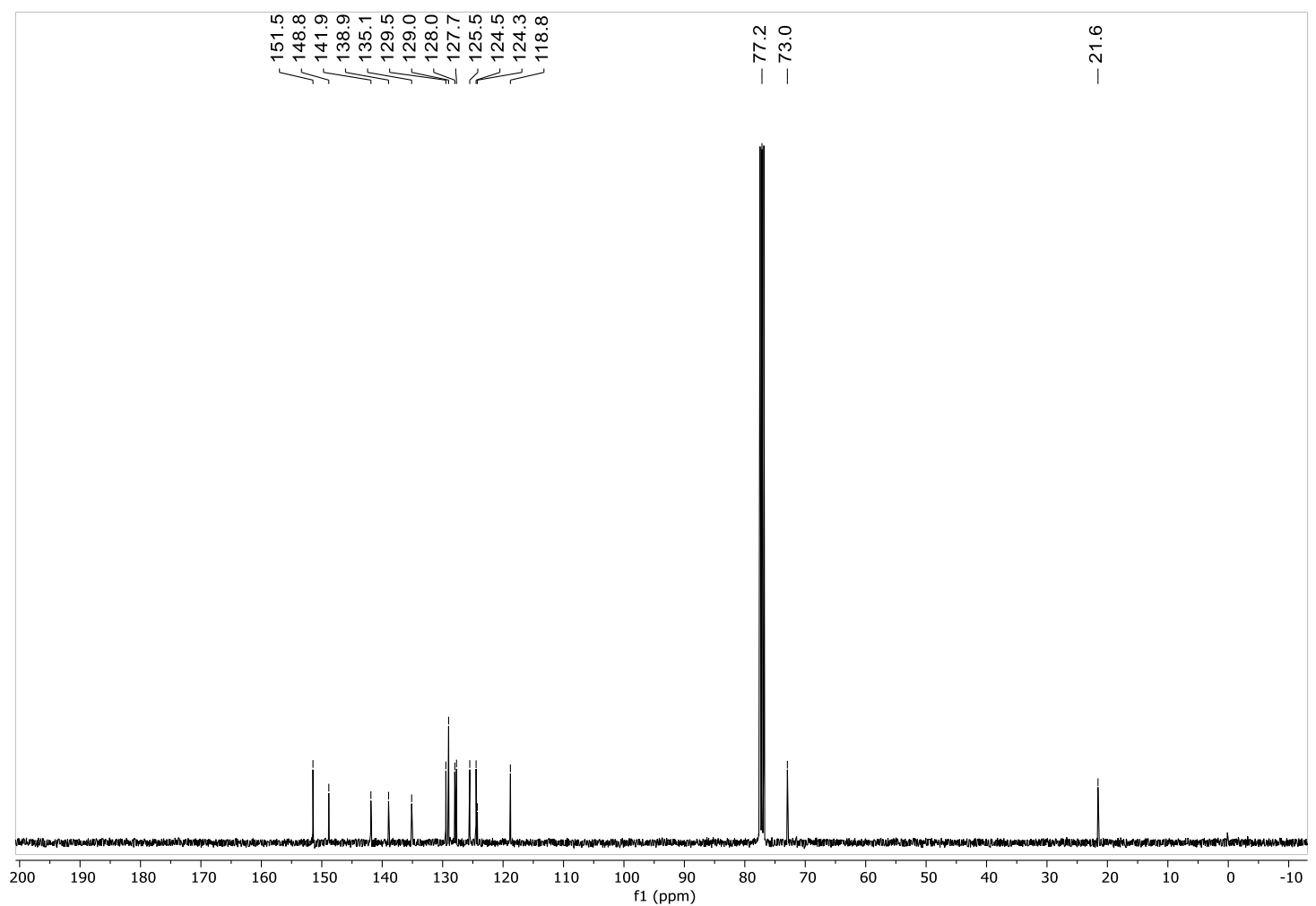


${ }^{1} \mathrm{H}$ NMR spectrum in MeOD-d $\mathrm{d}_{4}$ of compound 9d

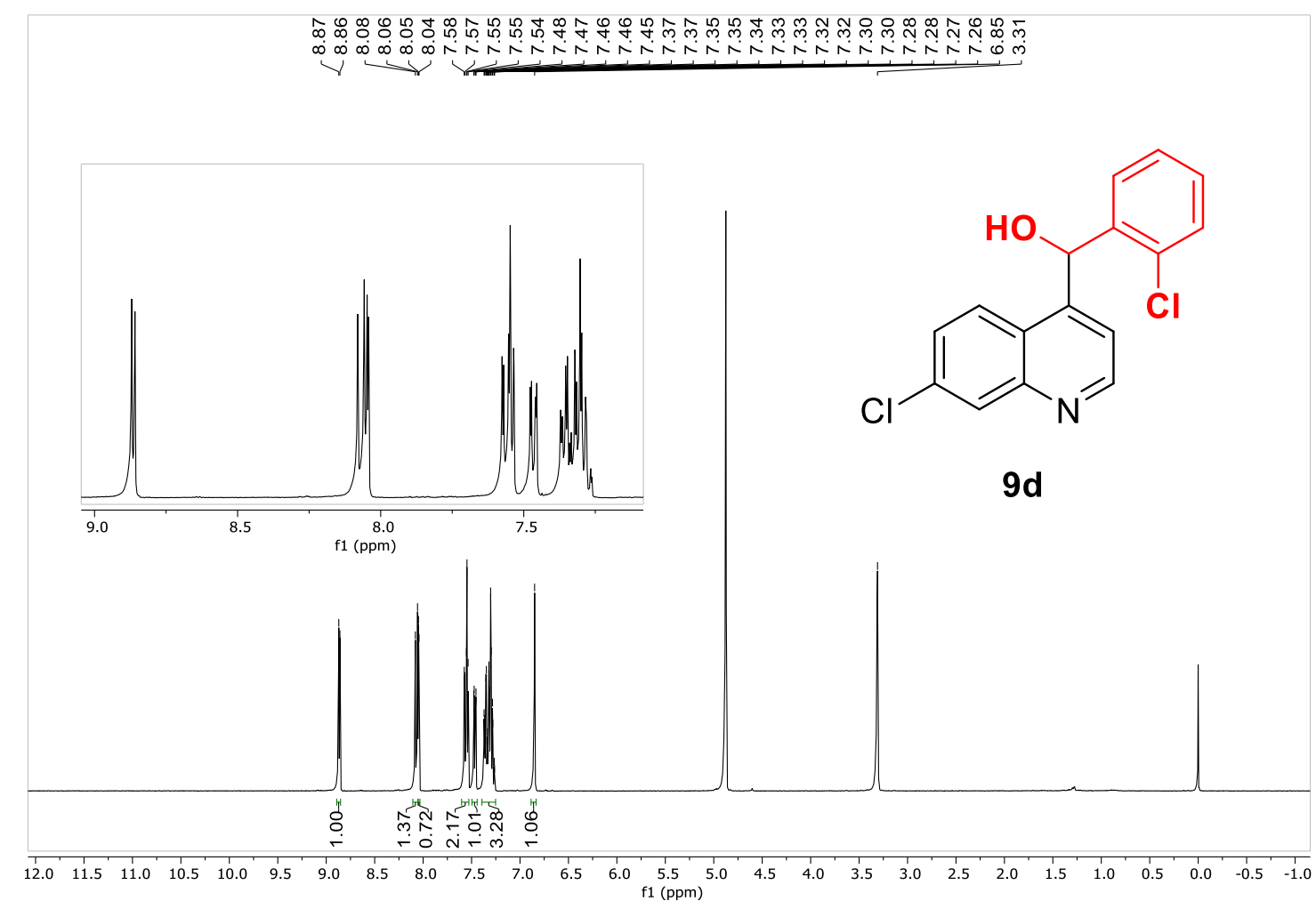

${ }^{13} \mathrm{C}\left\{{ }^{1} \mathrm{H}\right\}$ NMR spectrum in MeOD-d $\mathrm{d}_{4}$ of compound 9d

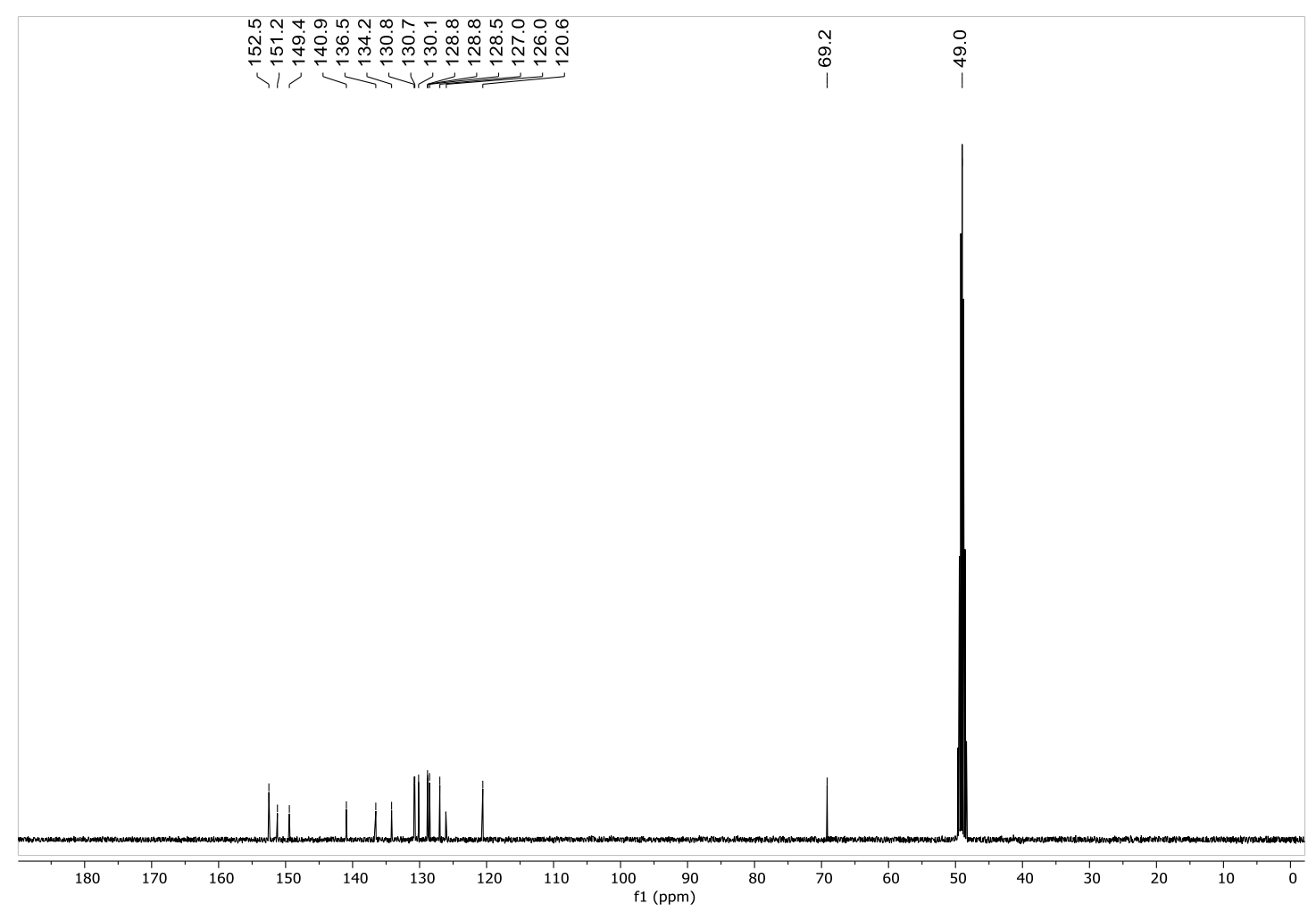


${ }^{1} \mathrm{H}$ NMR spectrum in DMSO- $\mathrm{d}_{6}$ of compound $9 \mathbf{e}$

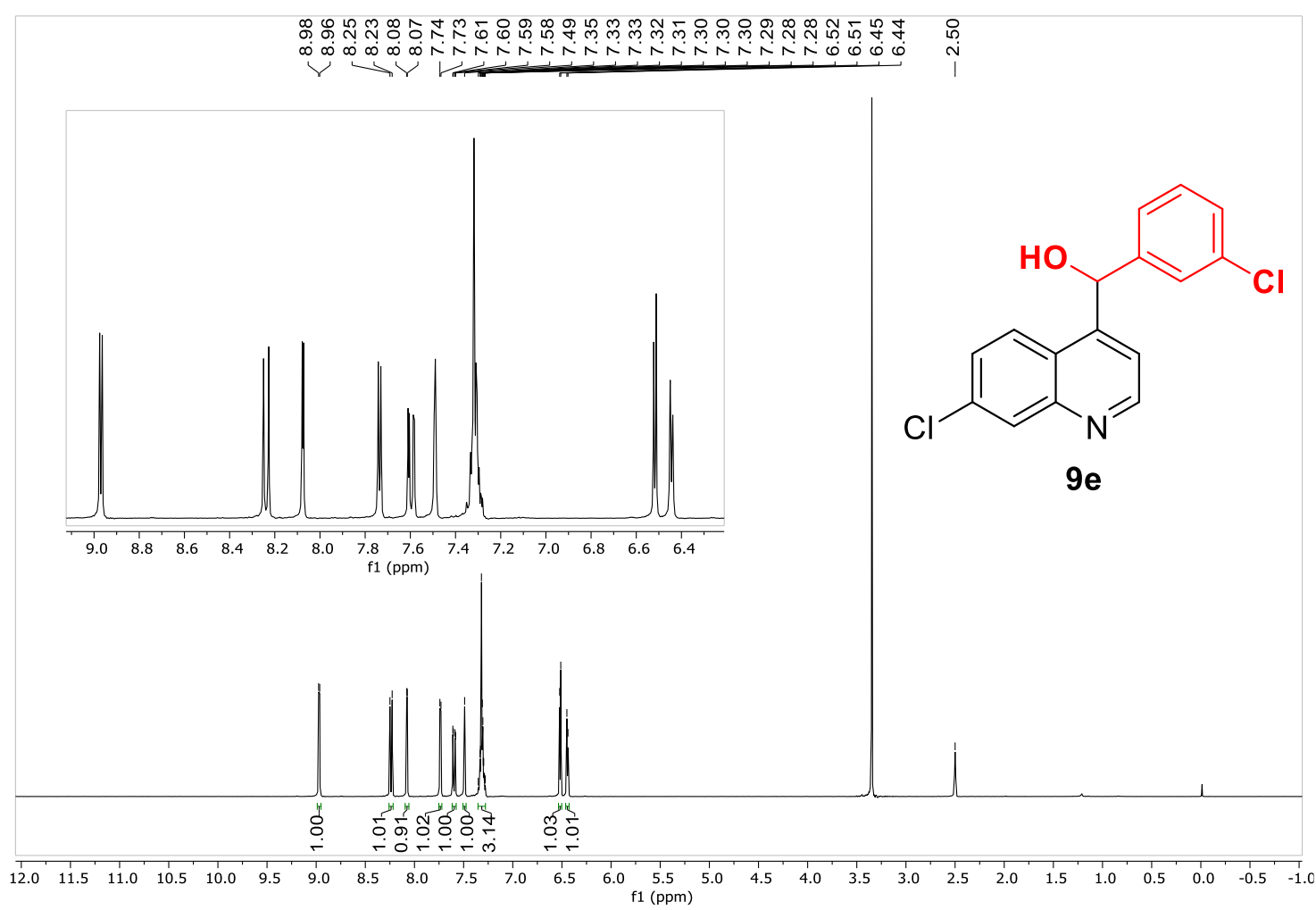

${ }^{13} \mathrm{C}\left\{{ }^{1} \mathrm{H}\right\}$ NMR spectrum in DMSO-d 6 of compound $9 e$

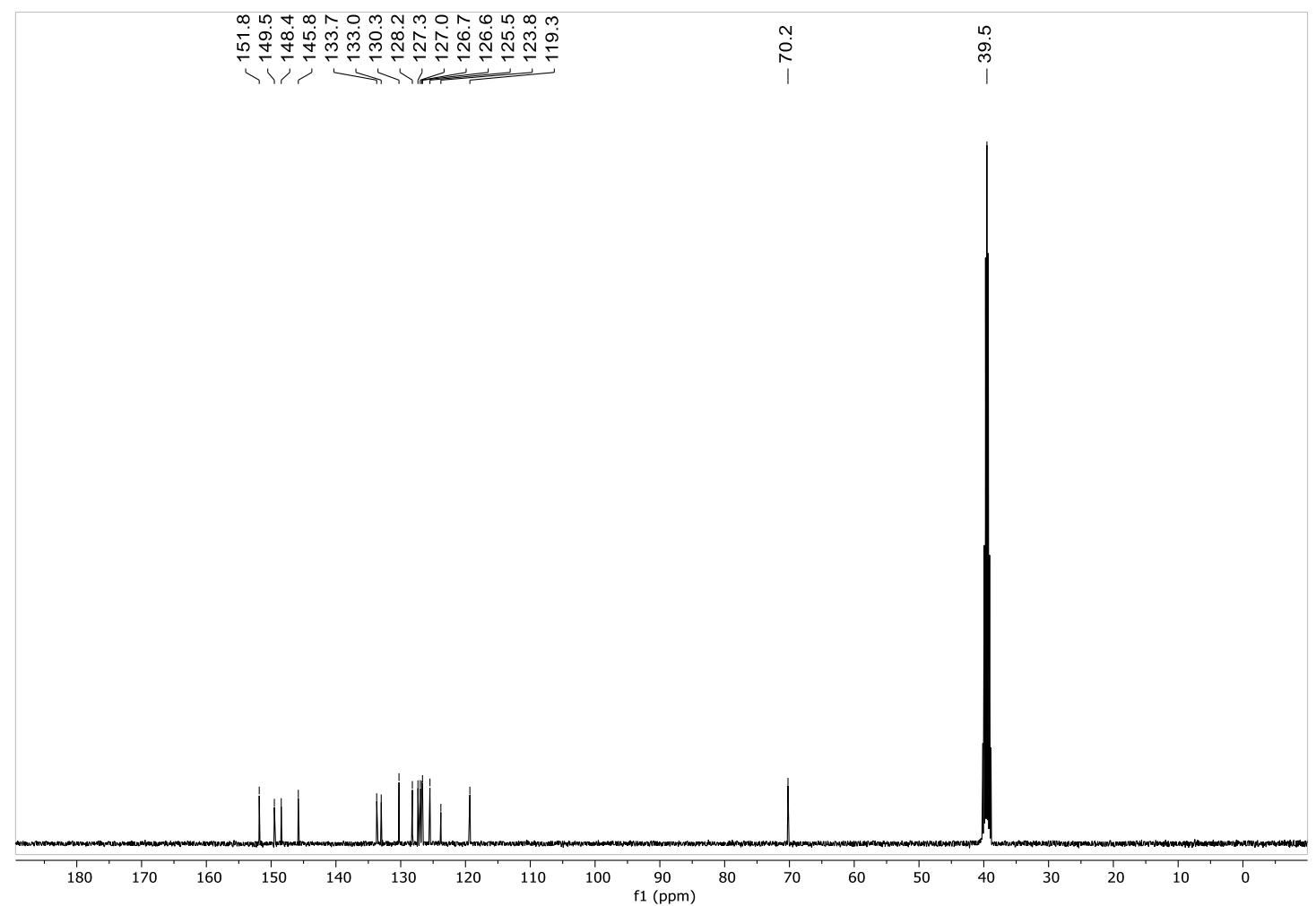


${ }^{1} \mathrm{H}$ NMR spectrum in $\mathrm{CDCl}_{3}$ of compound $\mathbf{9 f}$

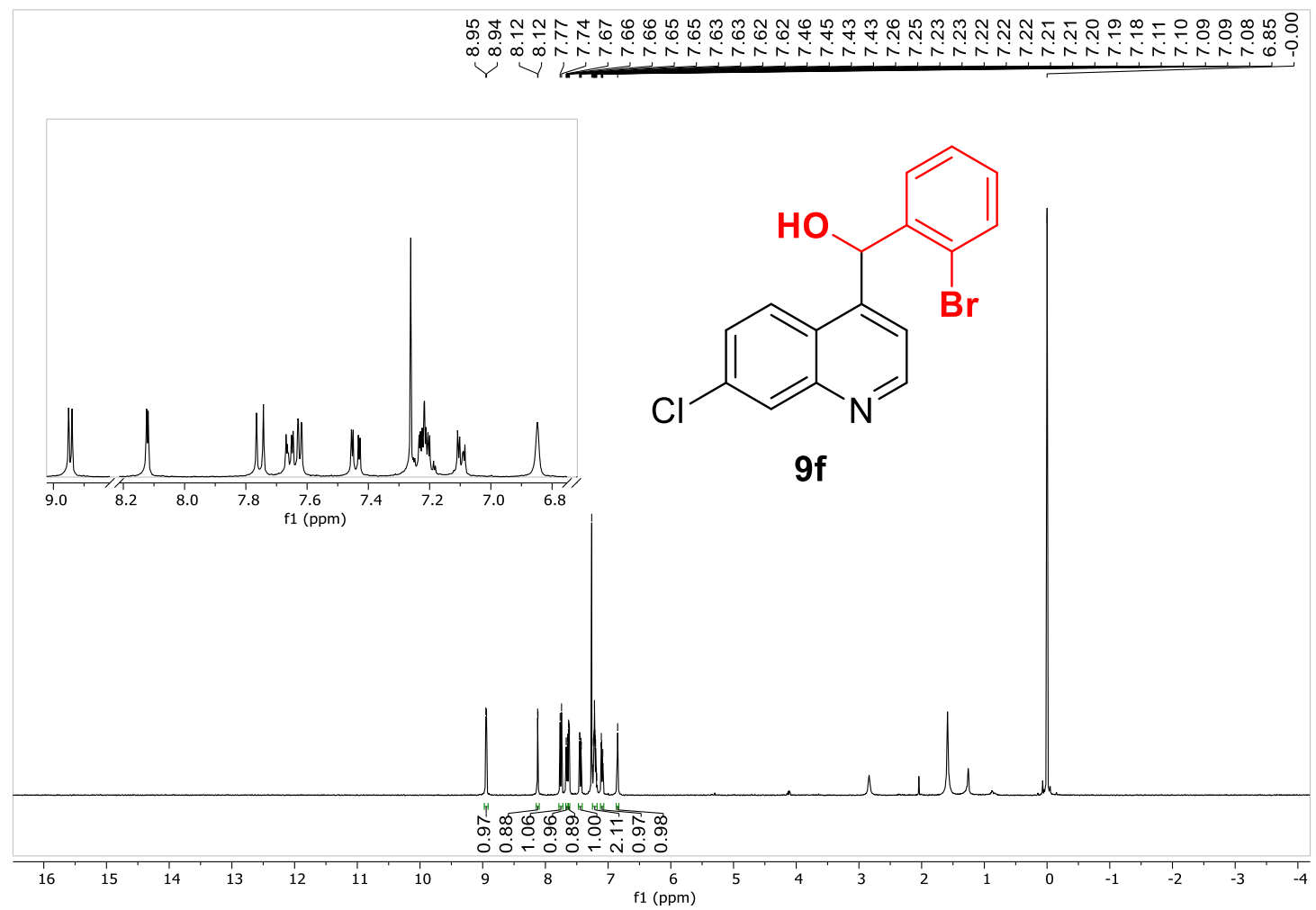

${ }^{13} \mathrm{C}\left\{{ }^{1} \mathrm{H}\right\}$ NMR spectrum in DMSO- $\mathrm{d}_{6}$ of compound $9 \mathrm{f}$

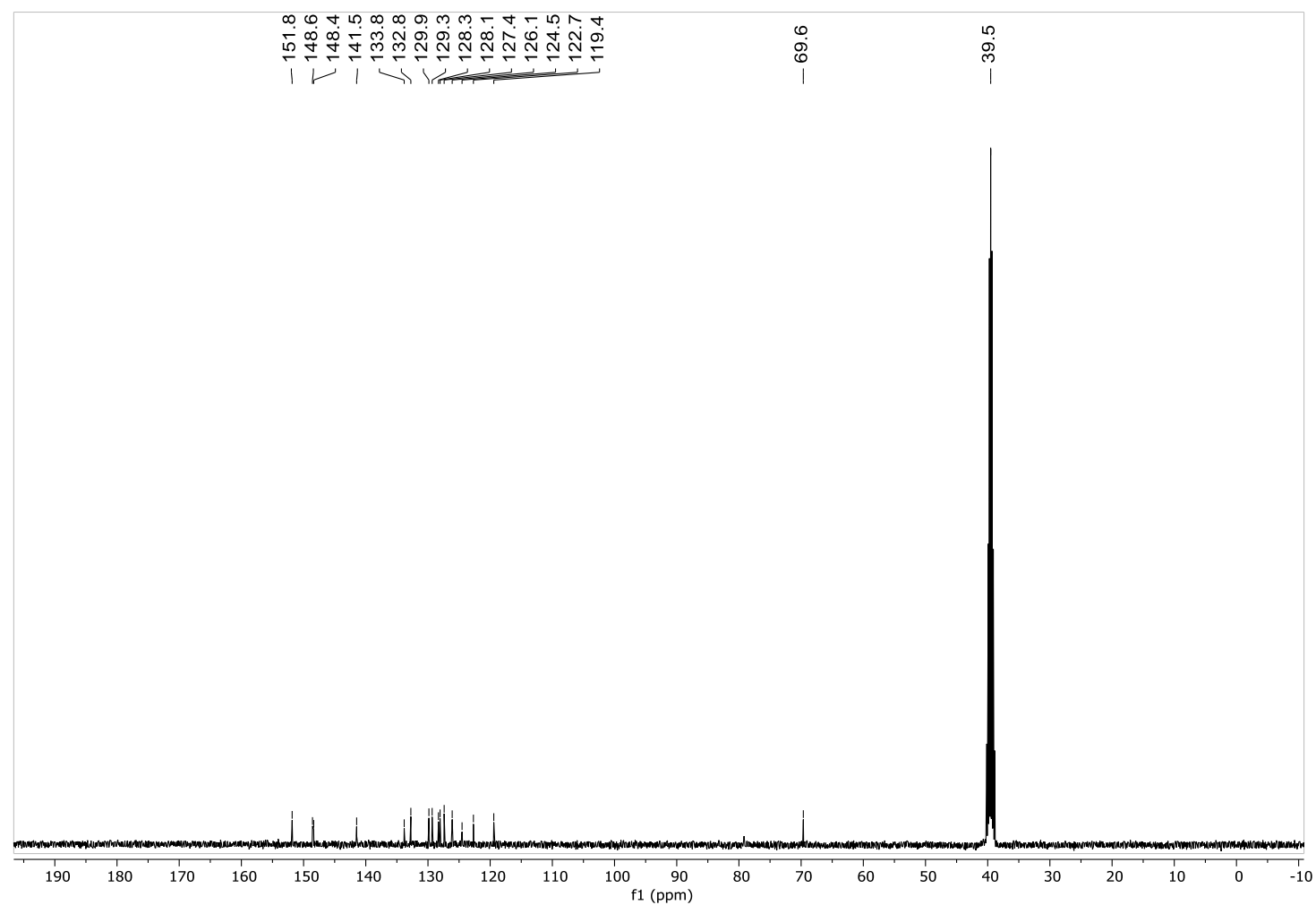


${ }^{1} \mathrm{H}$ NMR spectrum in DMSO- $\mathrm{d}_{6}$ of compound $9 \mathrm{~g}$

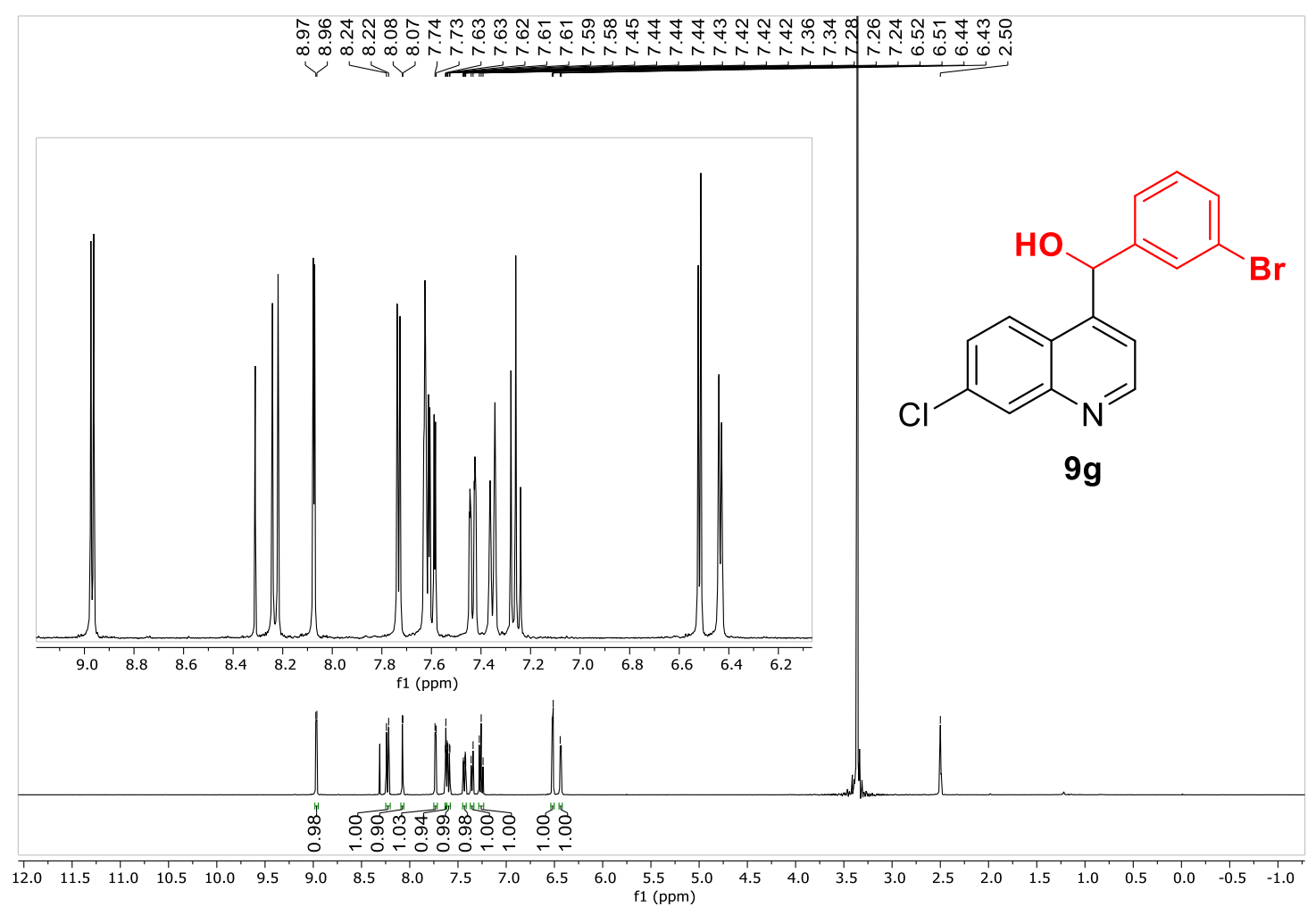

${ }^{13} \mathrm{C}\left\{{ }^{1} \mathrm{H}\right\}$ NMR spectrum in DMSO-d 6 of compound $9 \mathrm{~g}$

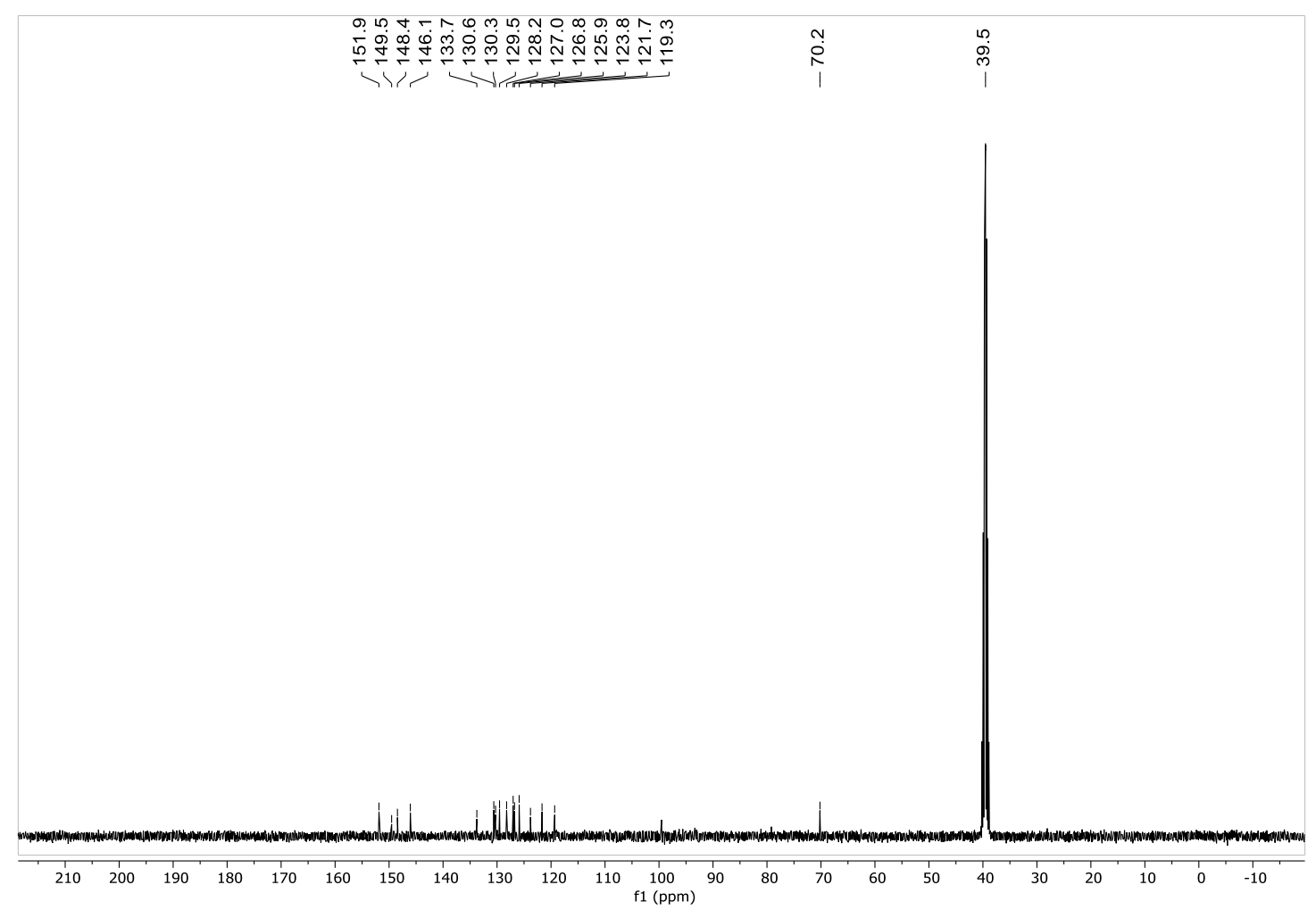


${ }^{1} \mathrm{H}$ NMR spectrum in $\mathrm{CDCl}_{3}$ of compound $\mathbf{9 h}$

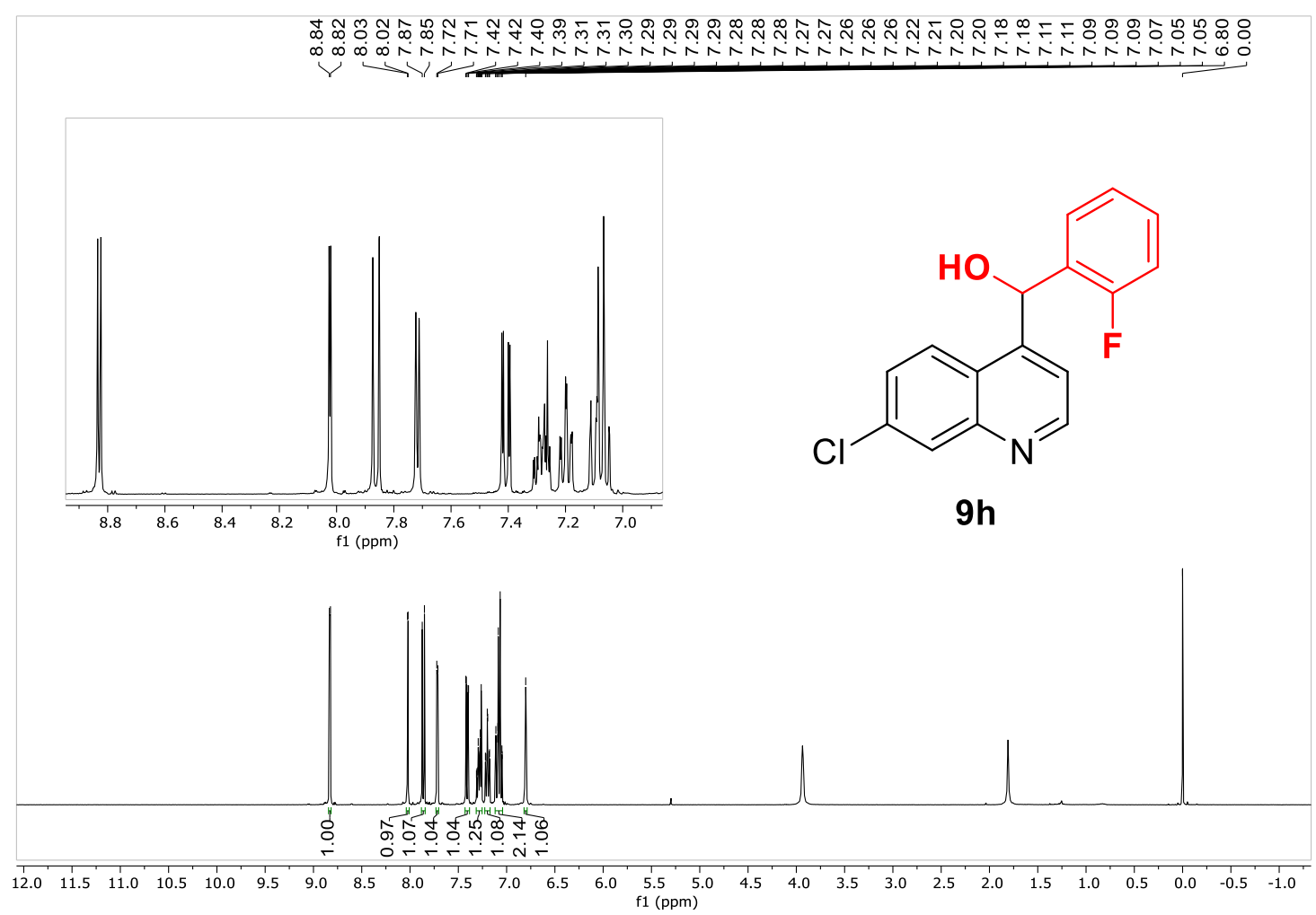

${ }^{13} \mathrm{C}\left\{{ }^{1} \mathrm{H}\right\}$ NMR spectrum in $\mathrm{CDCl}_{3}$ of compound $\mathbf{9 h}$

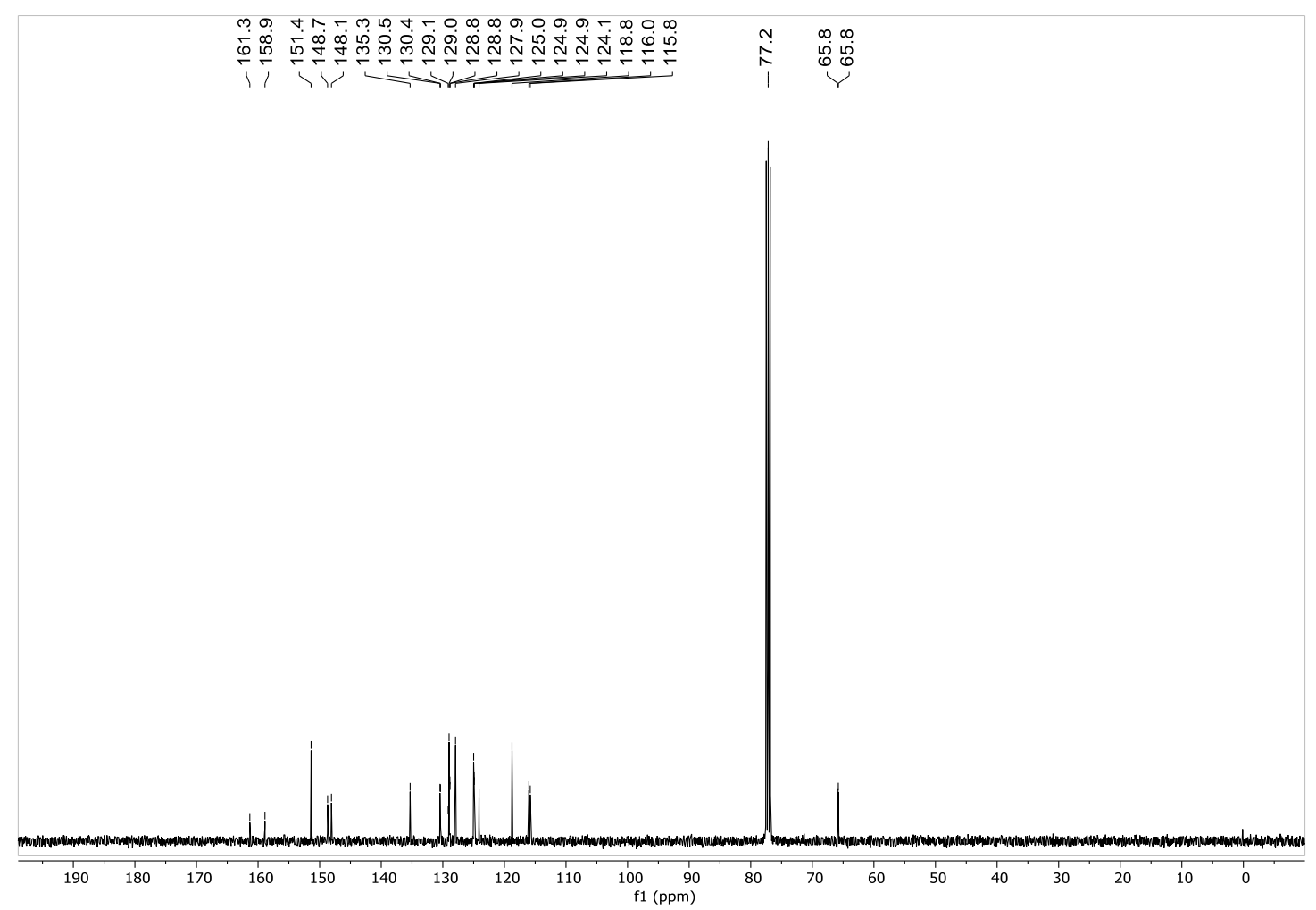


${ }^{1} \mathrm{H}$ NMR spectrum in MeOD- $\mathrm{d}_{4}$ of compound $\mathbf{9 i}$

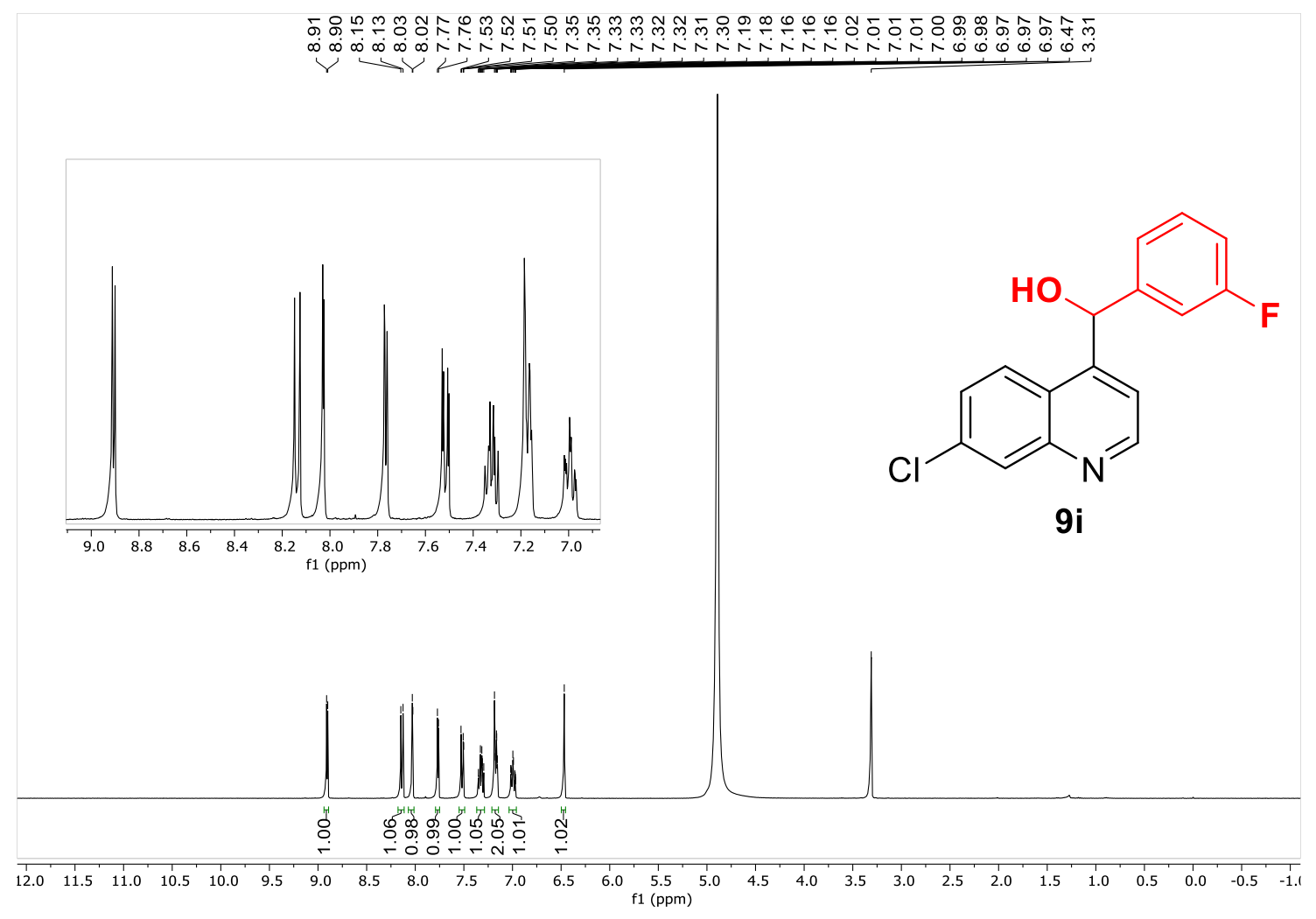

${ }^{13} \mathrm{C}\left\{{ }^{1} \mathrm{H}\right\}$ NMR spectrum in MeOD- $\mathrm{d}_{4}$ of compound $9 \mathrm{i}$

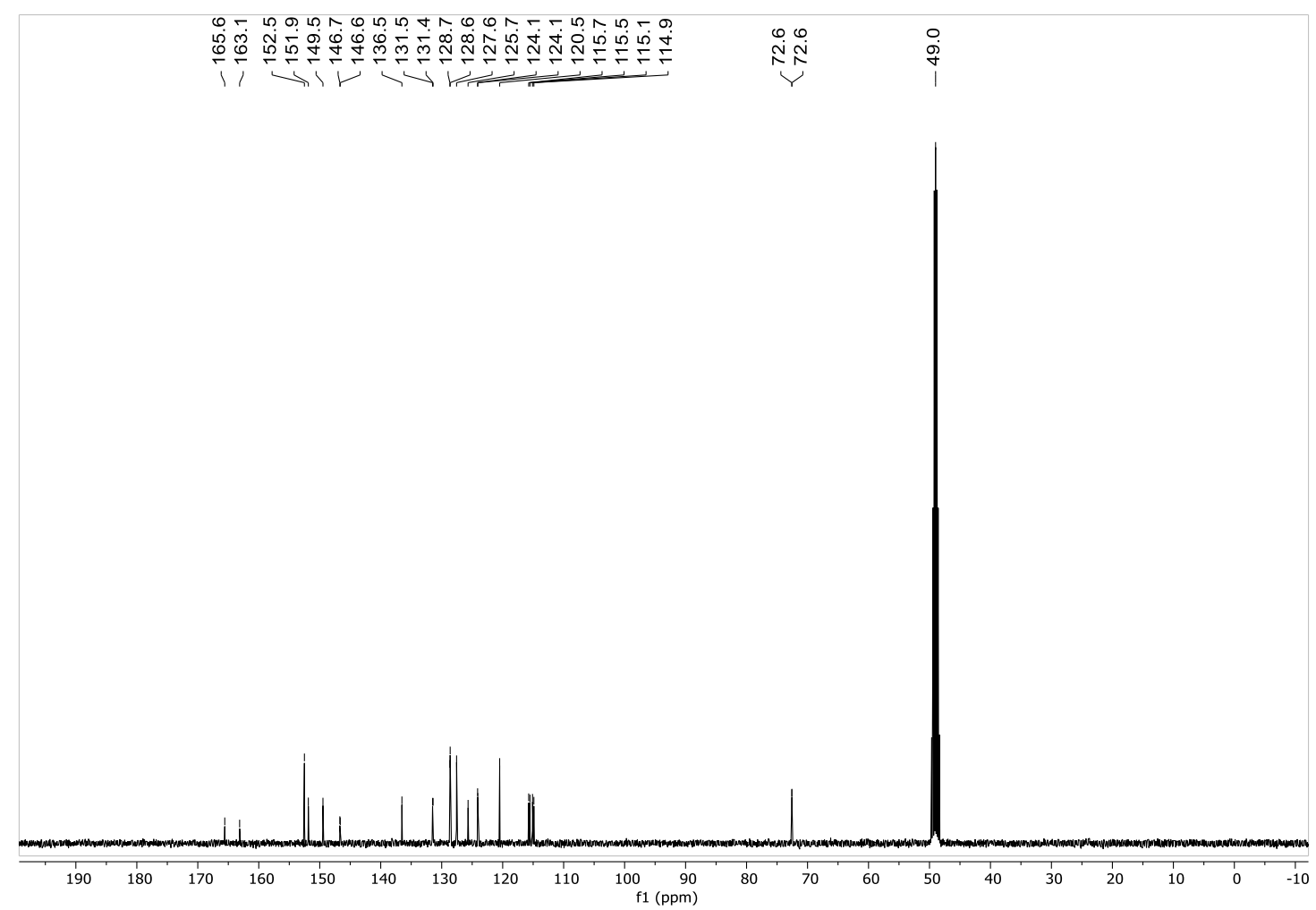


${ }^{1} \mathrm{H}$ NMR spectrum in $\mathrm{CDCl}_{3}$ of compound $\mathbf{9 j}$

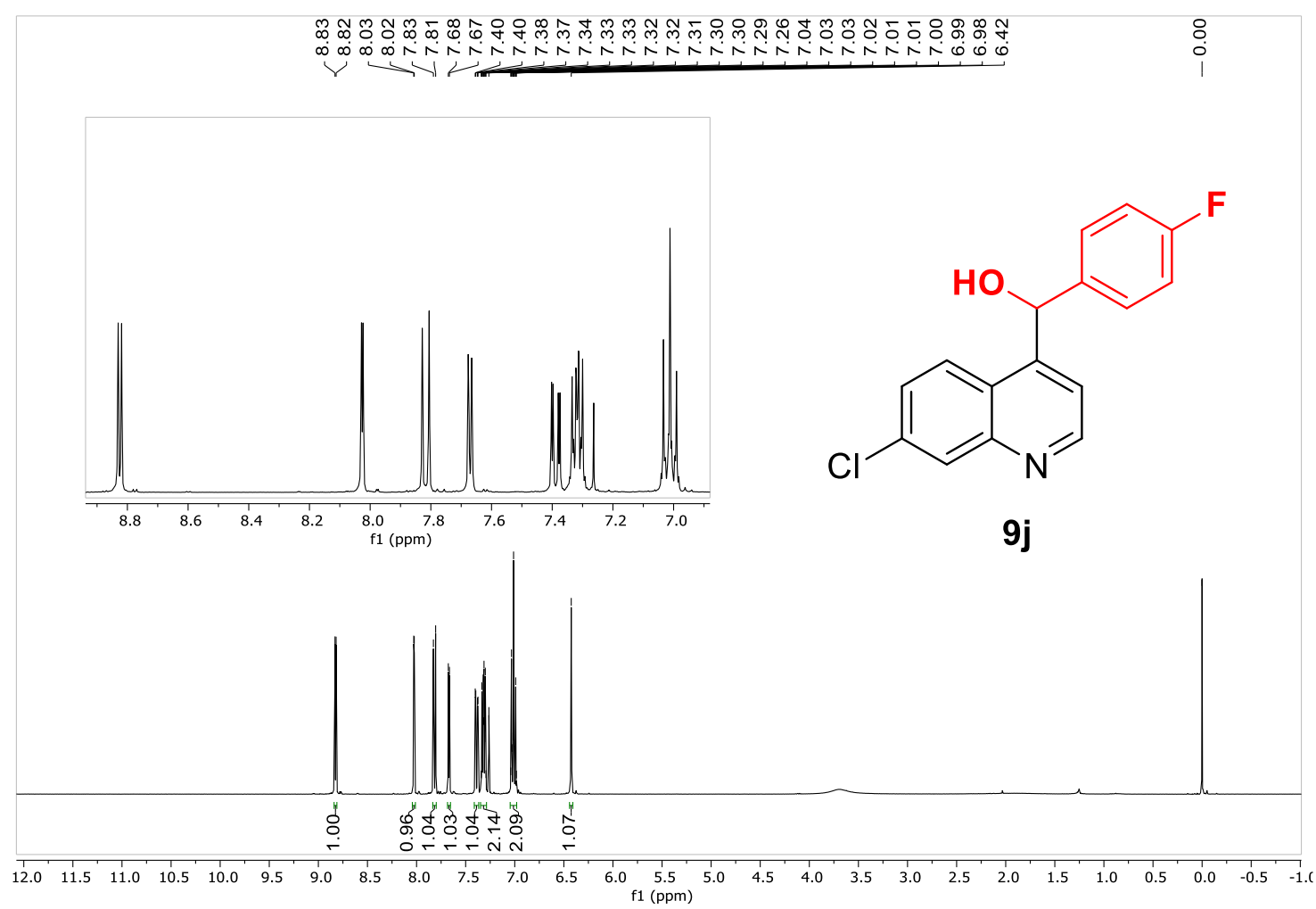

${ }^{13} \mathrm{C}\left\{{ }^{1} \mathrm{H}\right\}$ NMR spectrum in $\mathrm{CDCl}_{3}$ of compound $\mathbf{9 j}$

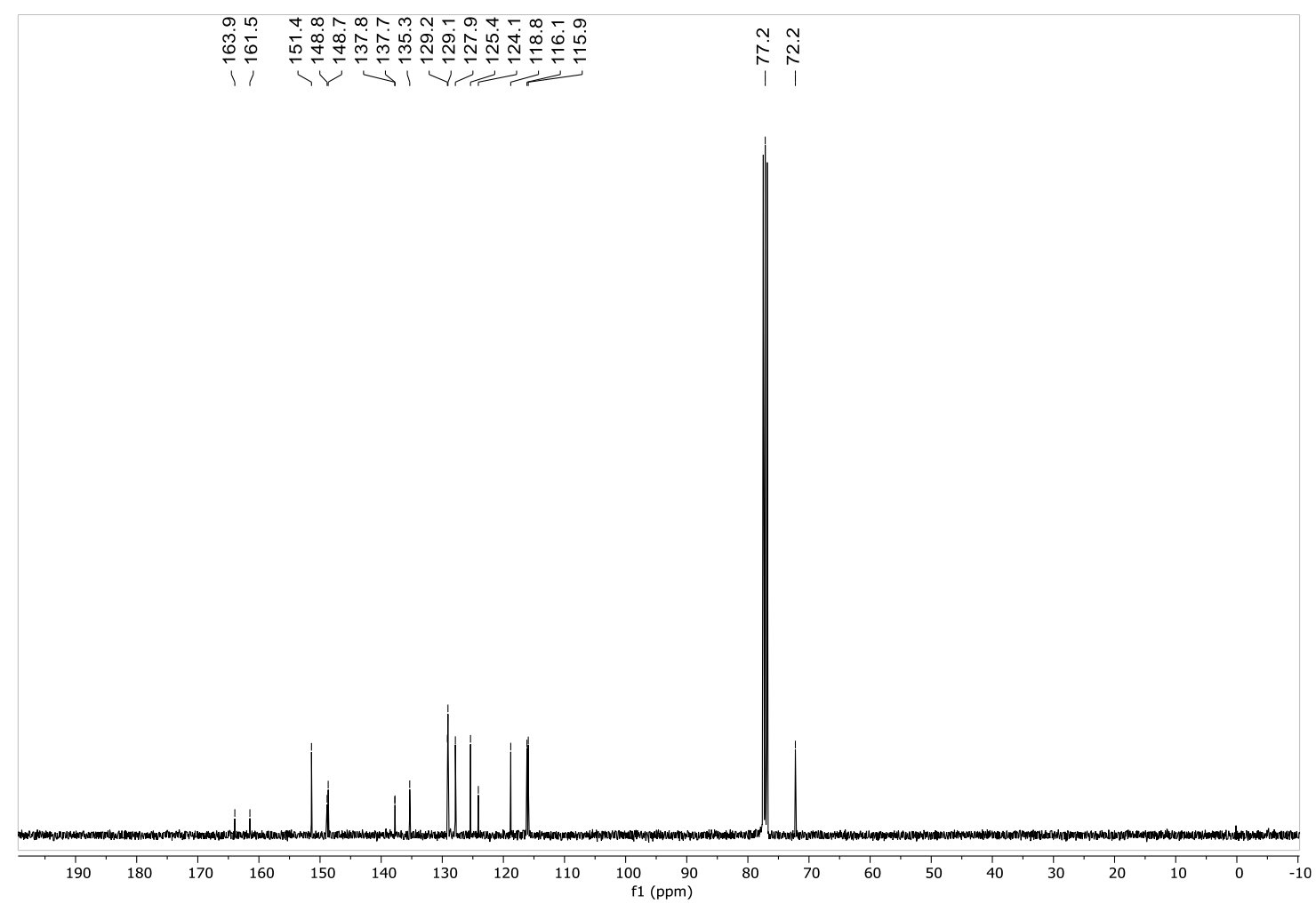


${ }^{1} \mathrm{H}$ NMR spectrum in DMSO-d $\mathrm{d}_{6}$ of compound 9k

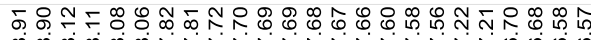

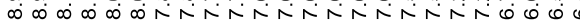

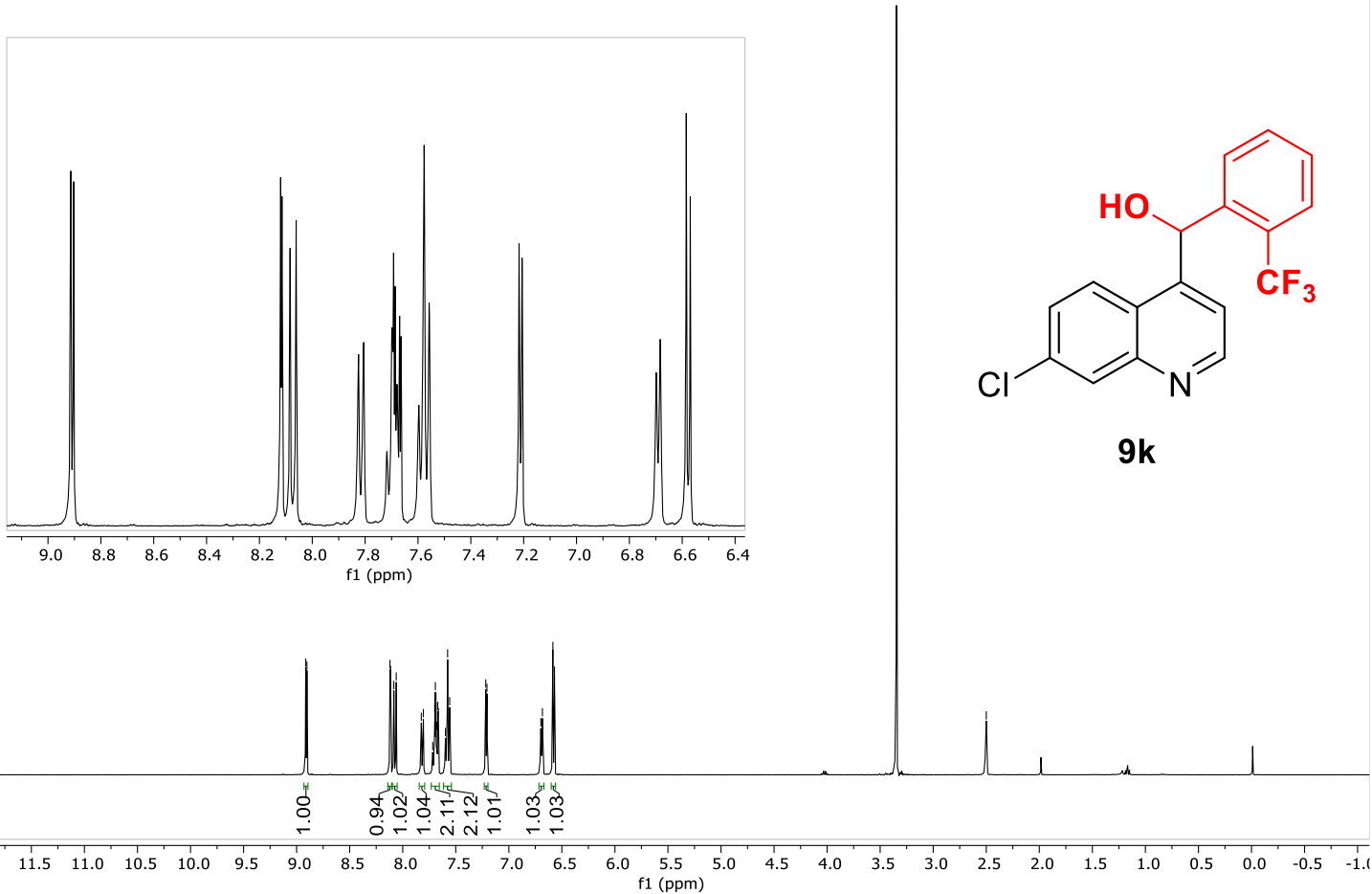

${ }^{13} \mathrm{C}\left\{{ }^{1} \mathrm{H}\right\}$ NMR spectrum in DMSO-d $\mathrm{d}_{6}$ of compound $9 \mathbf{k}$

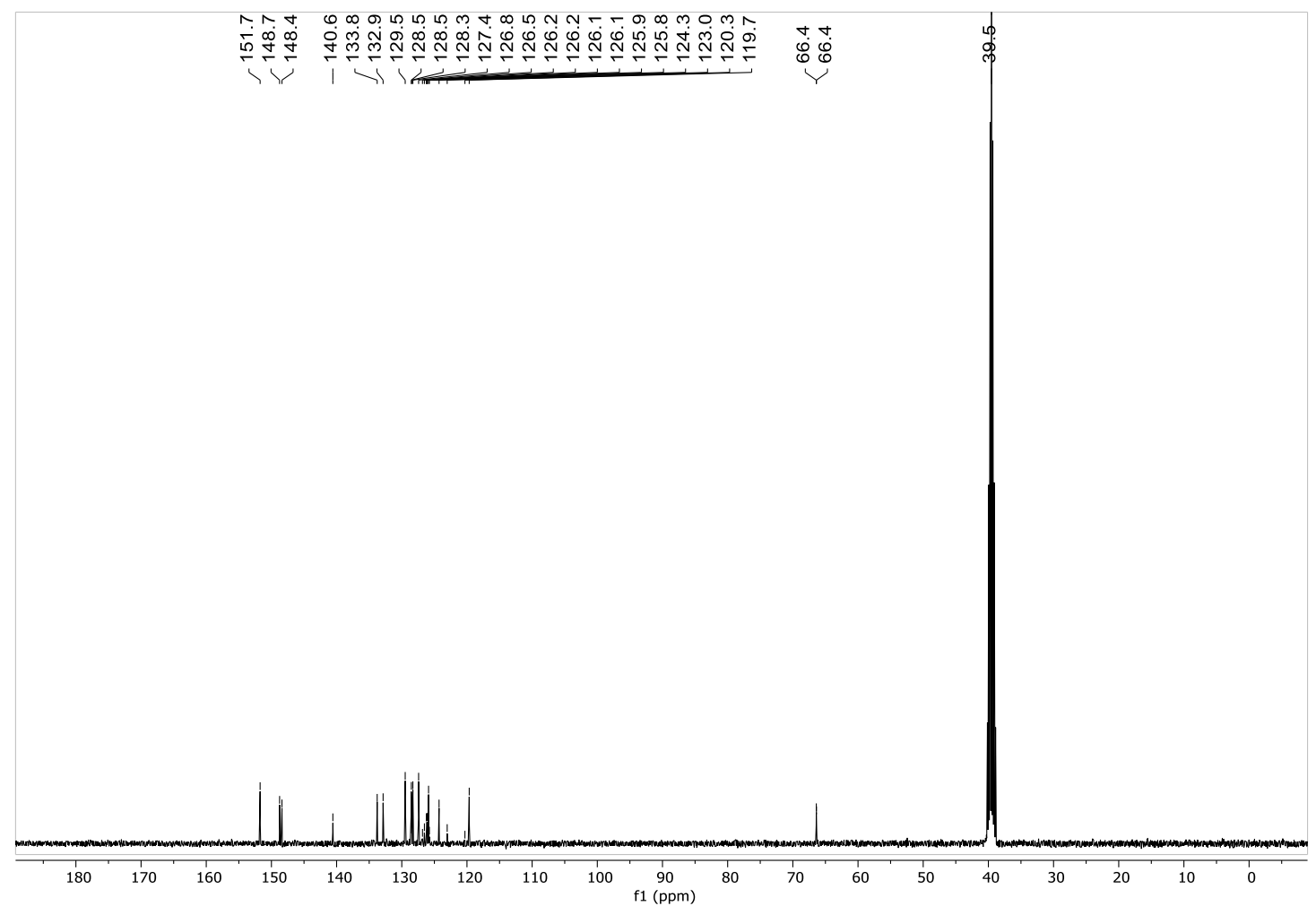


${ }^{1} \mathrm{H}$ NMR spectrum in DMSO- $\mathrm{d}_{6}$ of compound $9 \mathrm{I}$

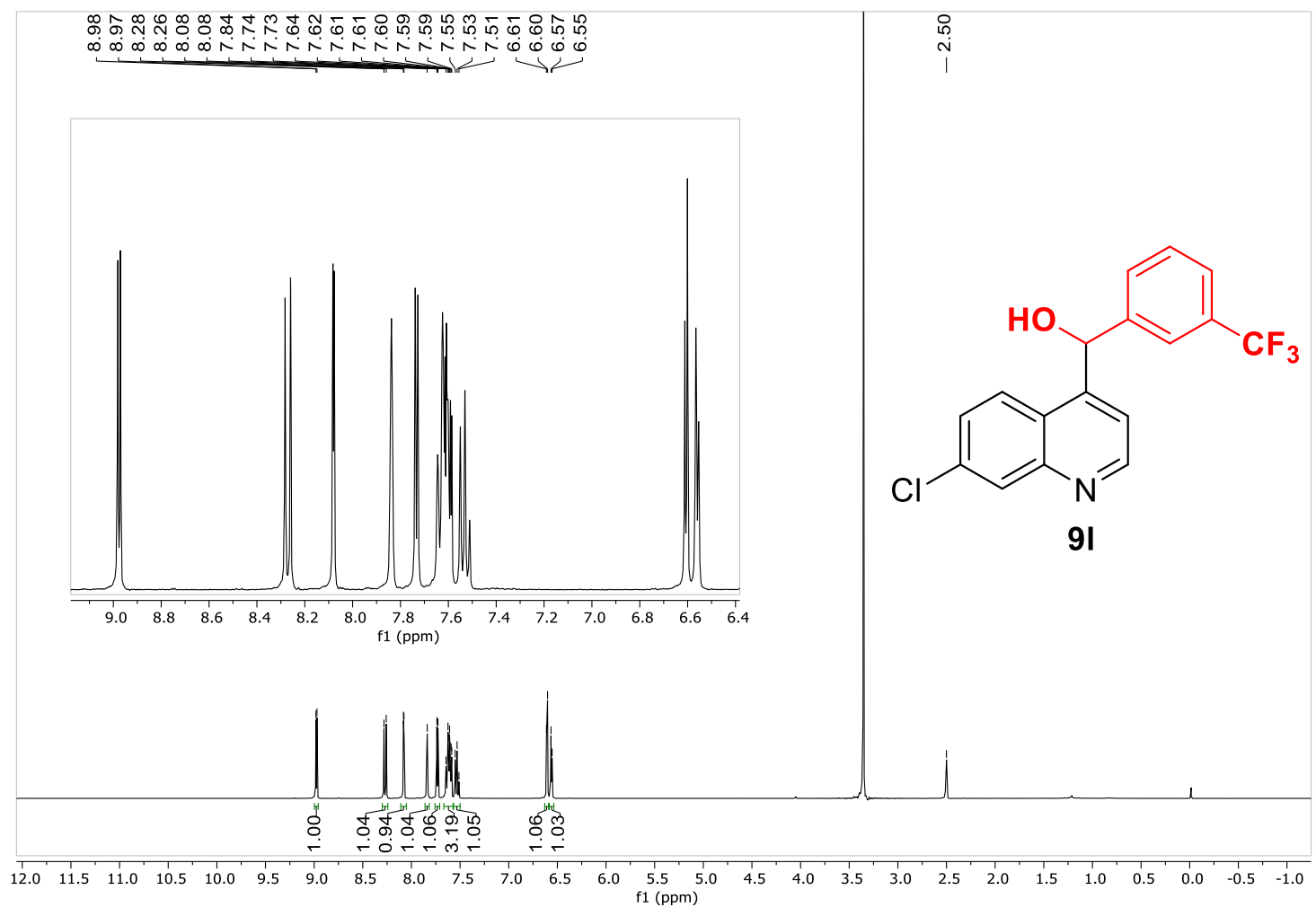

${ }^{13} \mathrm{C}\left\{{ }^{1} \mathrm{H}\right\}$ NMR spectrum in DMSO- $\mathrm{d}_{6}$ of compound 91

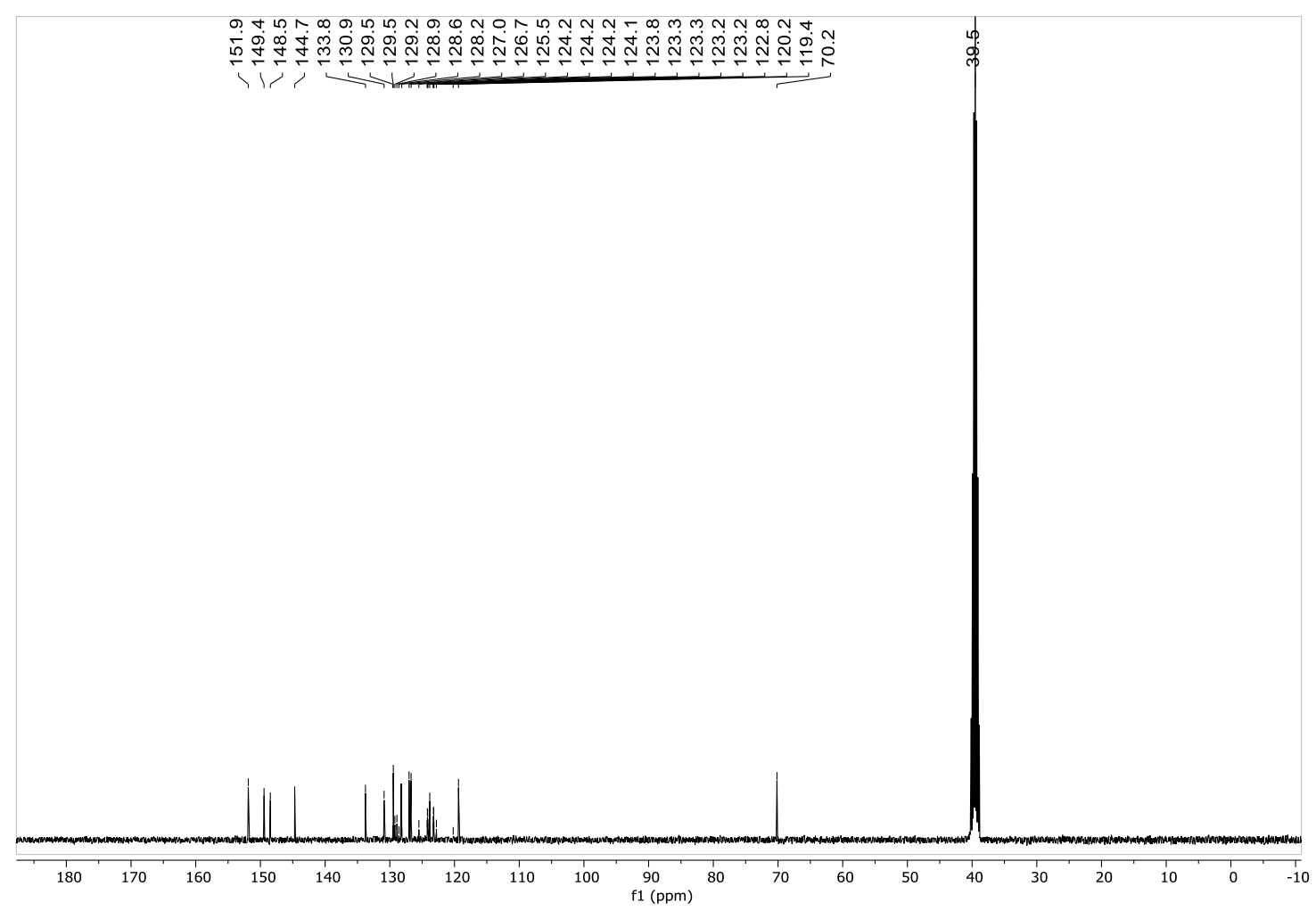


${ }^{1} \mathrm{H}$ NMR spectrum in DMSO- $\mathrm{d}_{6}$ of compound $\mathbf{9 m}$

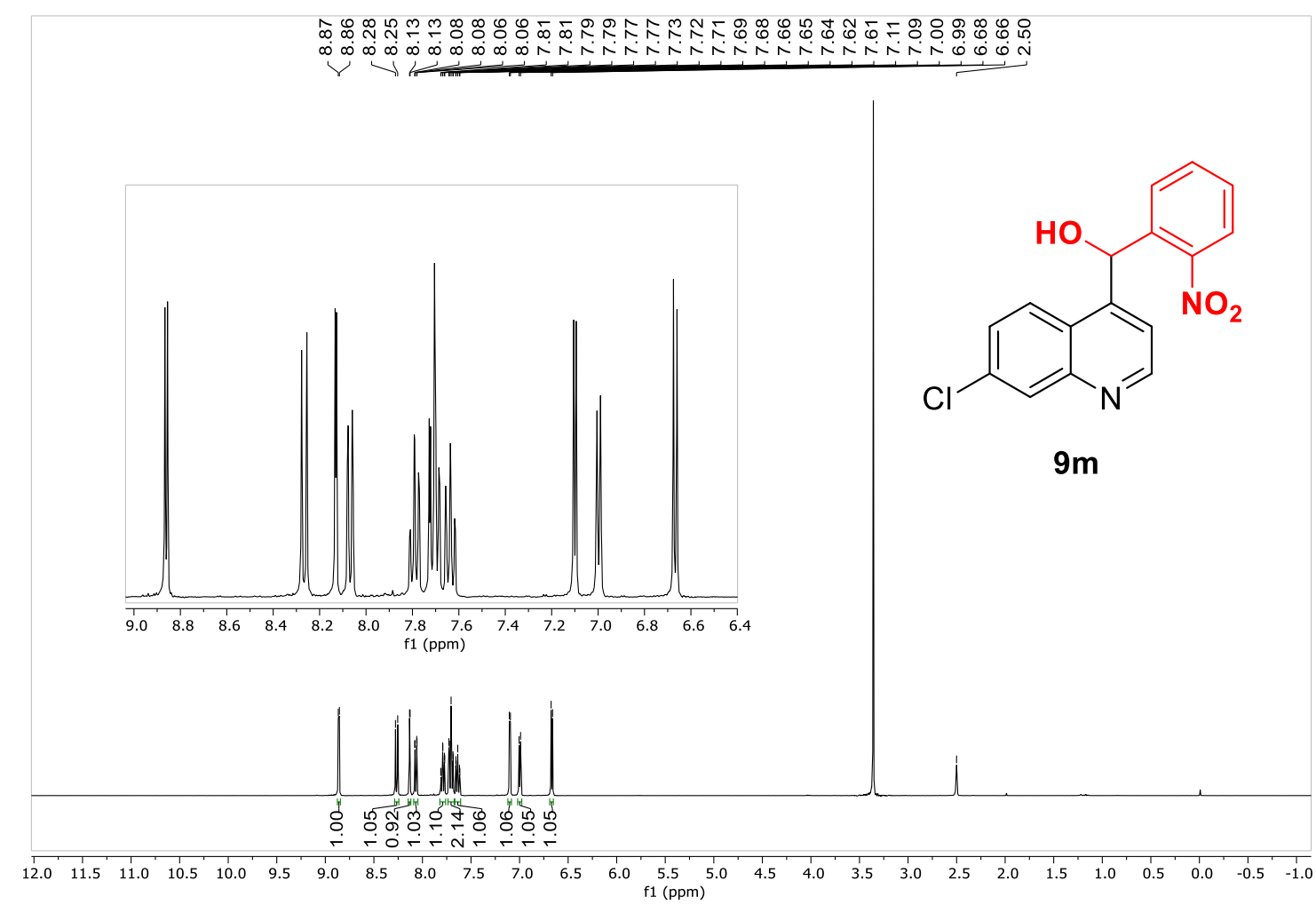

${ }^{13} \mathrm{C}\left\{{ }^{1} \mathrm{H}\right\}$ NMR spectrum in DMSO-d 6 of compound $9 \mathbf{m}$

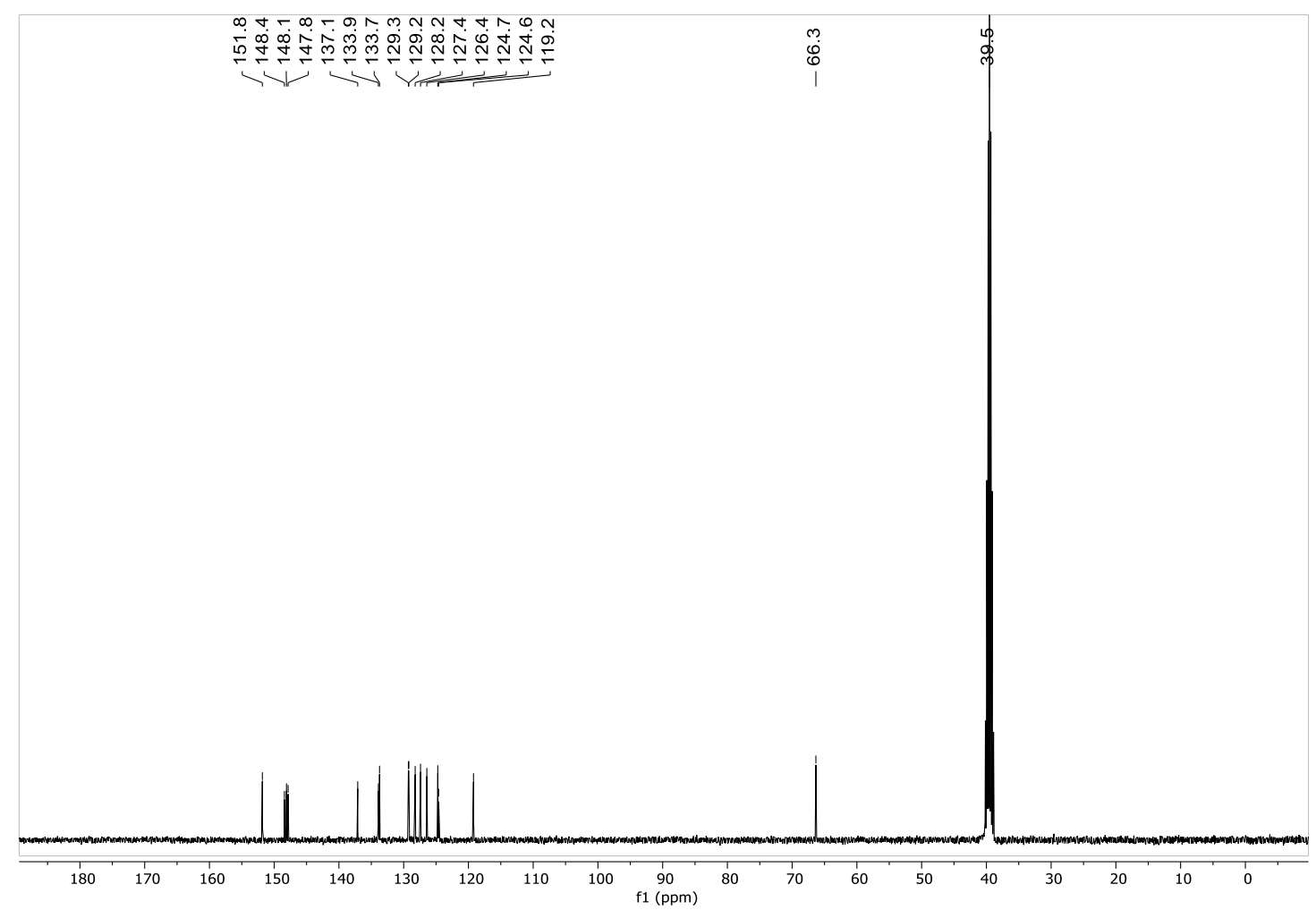


${ }^{1} \mathrm{H}$ NMR spectrum in DMSO- $\mathrm{d}_{6}$ of compound $9 \mathbf{n}$

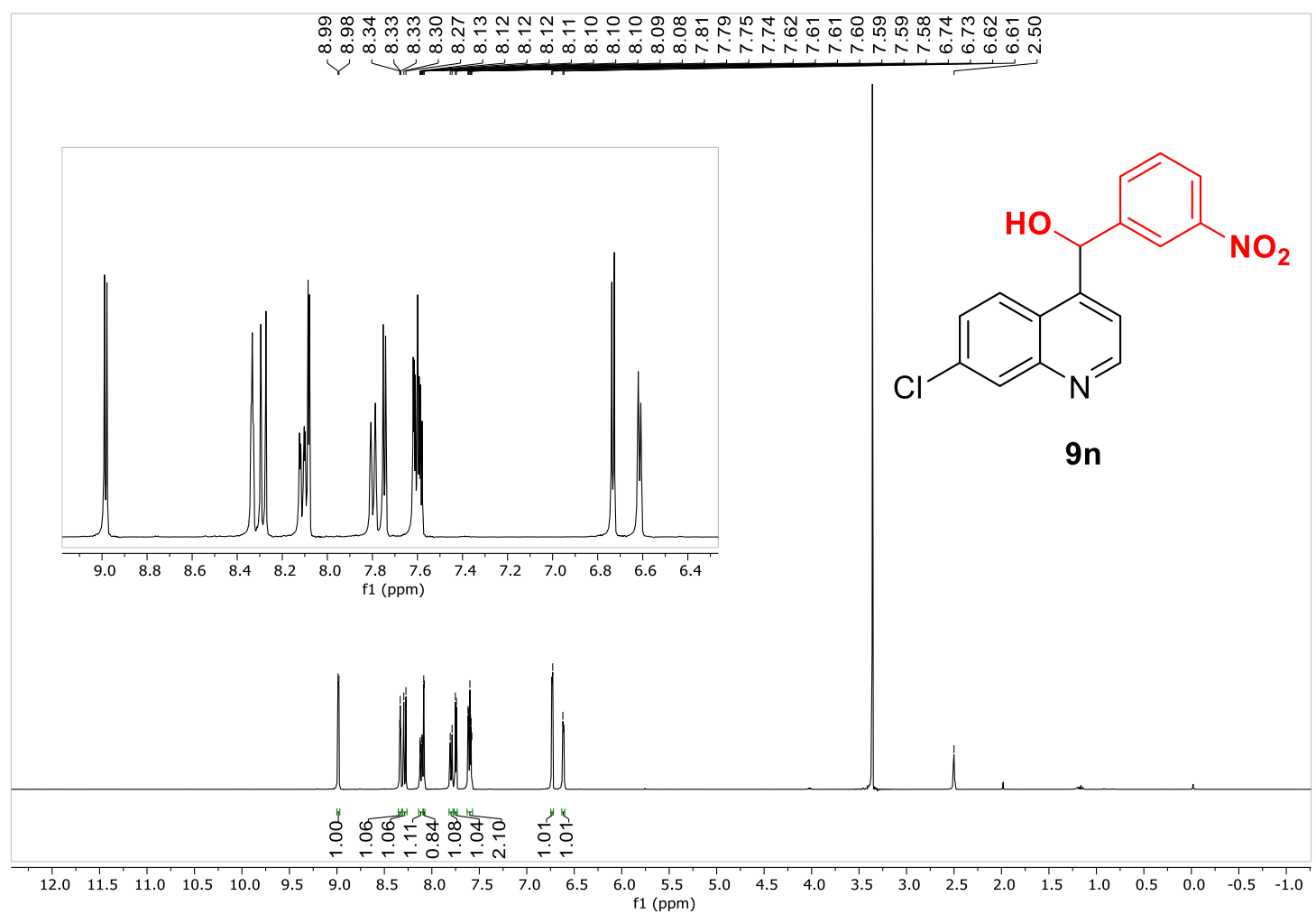

${ }^{13} \mathrm{C}\left\{{ }^{1} \mathrm{H}\right\}$ NMR spectrum in DMSO- $\mathrm{d}_{6}$ of compound 9n

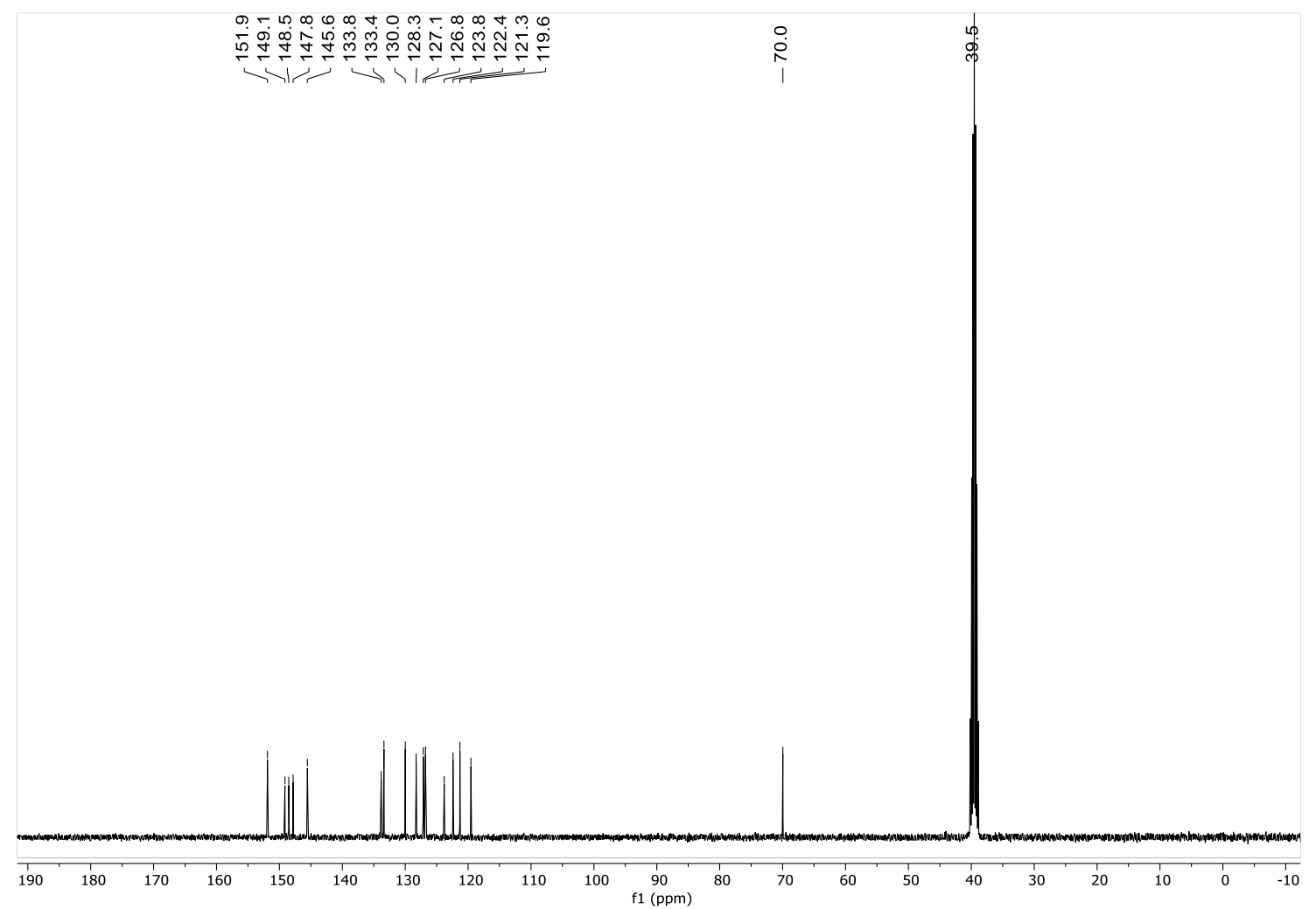


${ }^{1} \mathrm{H}$ NMR spectrum in DMSO- $\mathrm{d}_{6}$ of compound 90

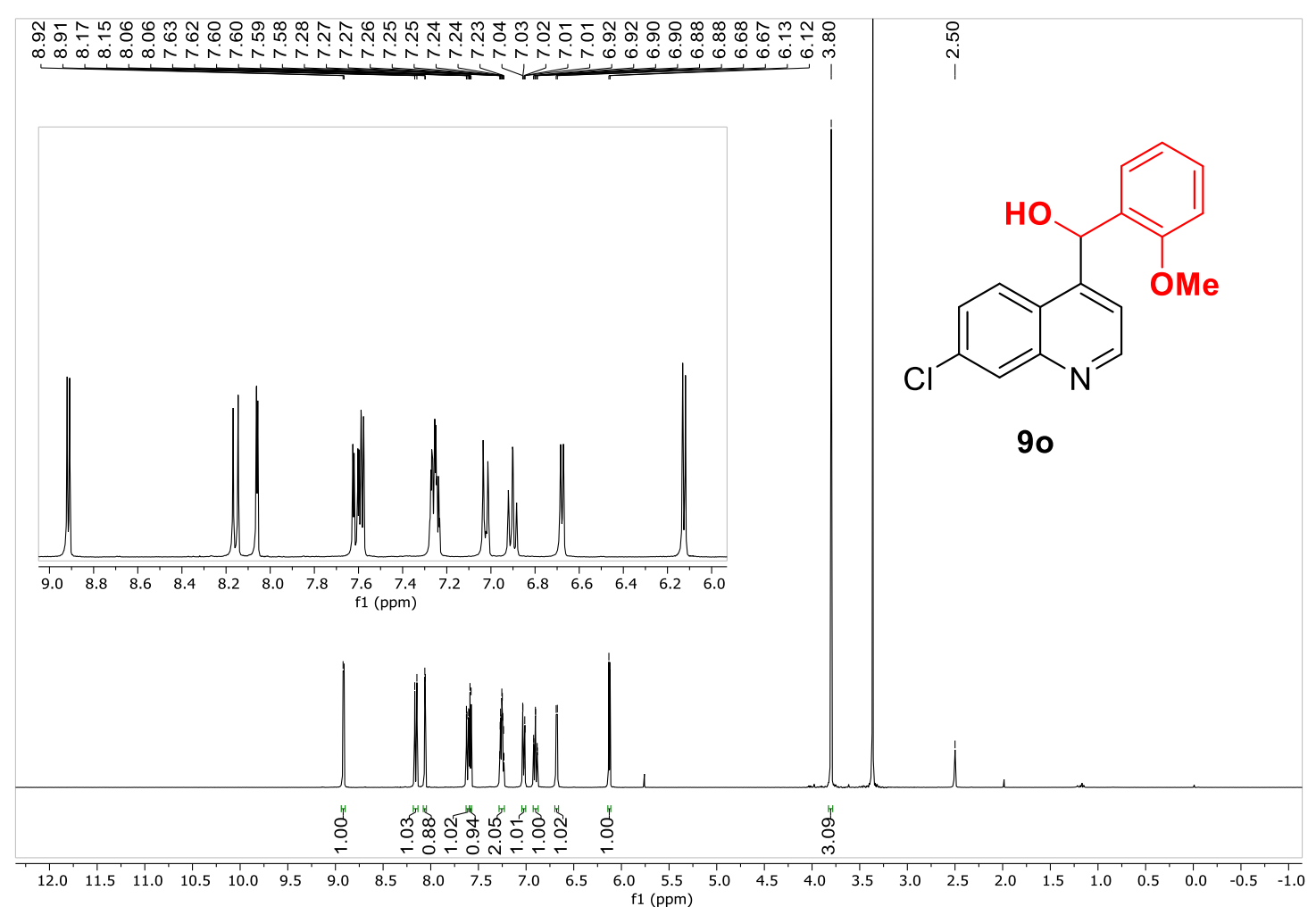

${ }^{13} \mathrm{C}\left\{{ }^{1} \mathrm{H}\right\}$ NMR spectrum in DMSO-d 6 of compound 9o

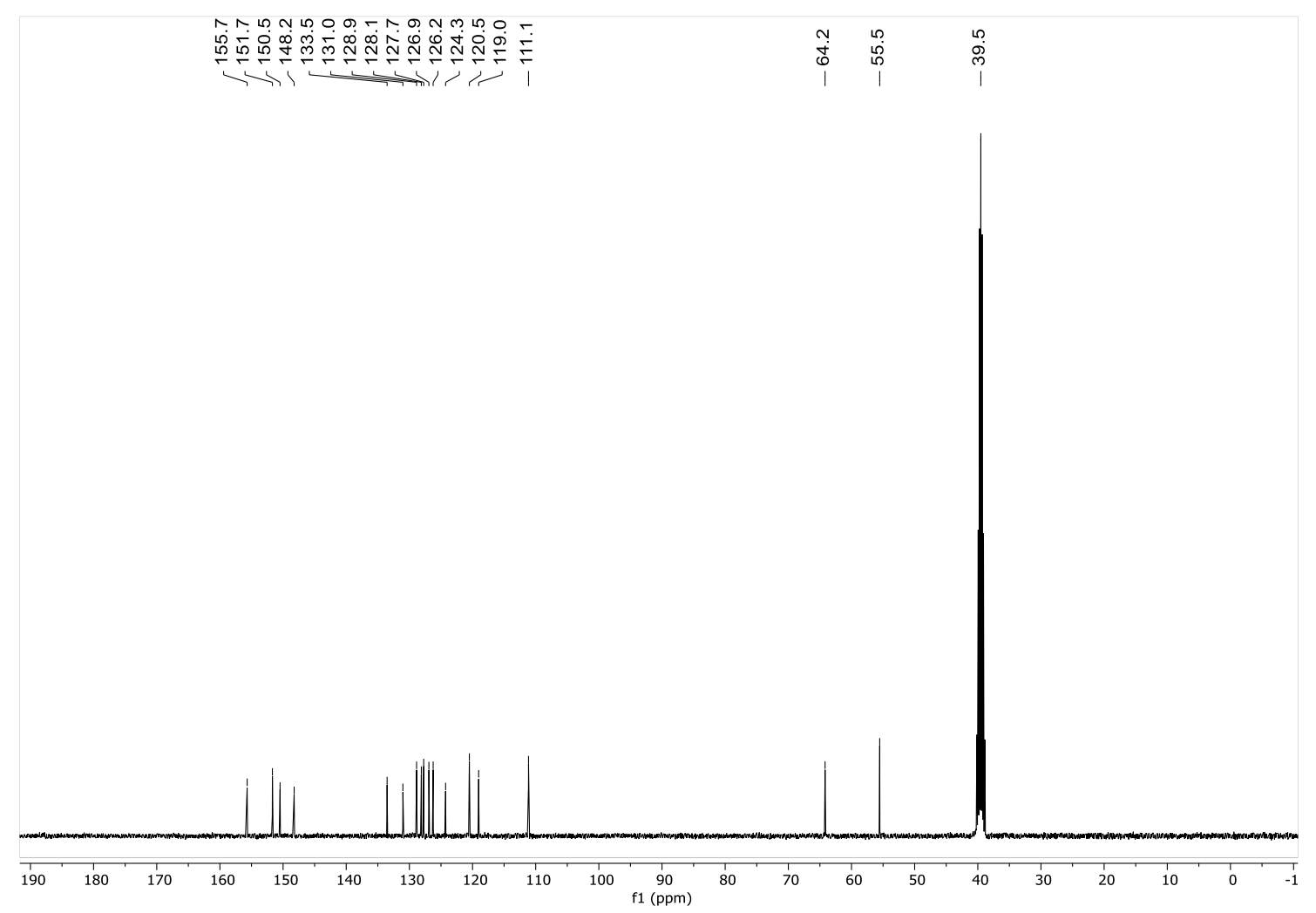


${ }^{1} \mathrm{H}$ NMR spectrum in MeOD- $\mathrm{d}_{4}$ of compound $\mathbf{9 p}$

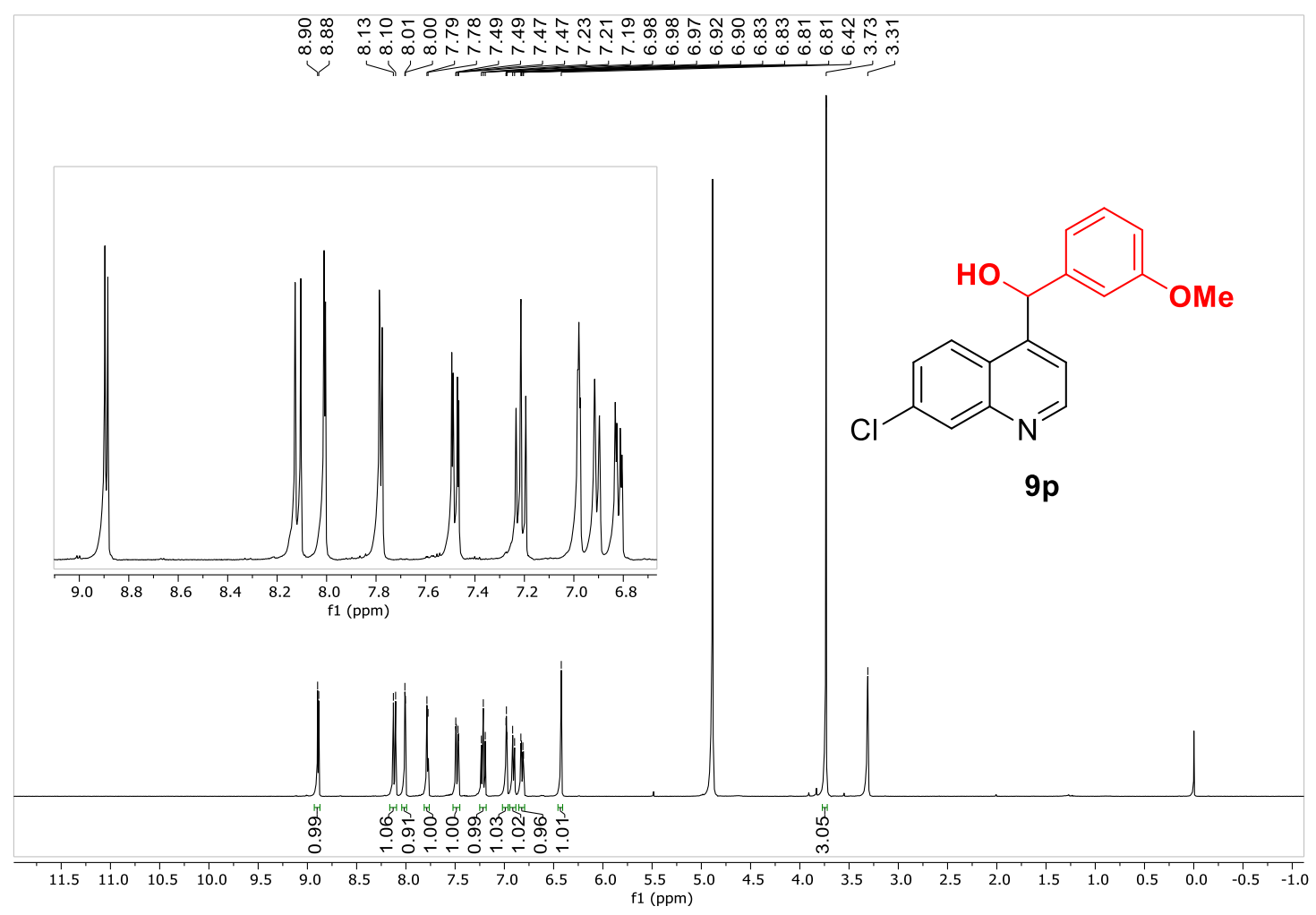

${ }^{13} \mathrm{C}\left\{{ }^{1} \mathrm{H}\right\}$ NMR spectrum in MeOD-d $\mathrm{d}_{4}$ of compound $\mathbf{9 p}$

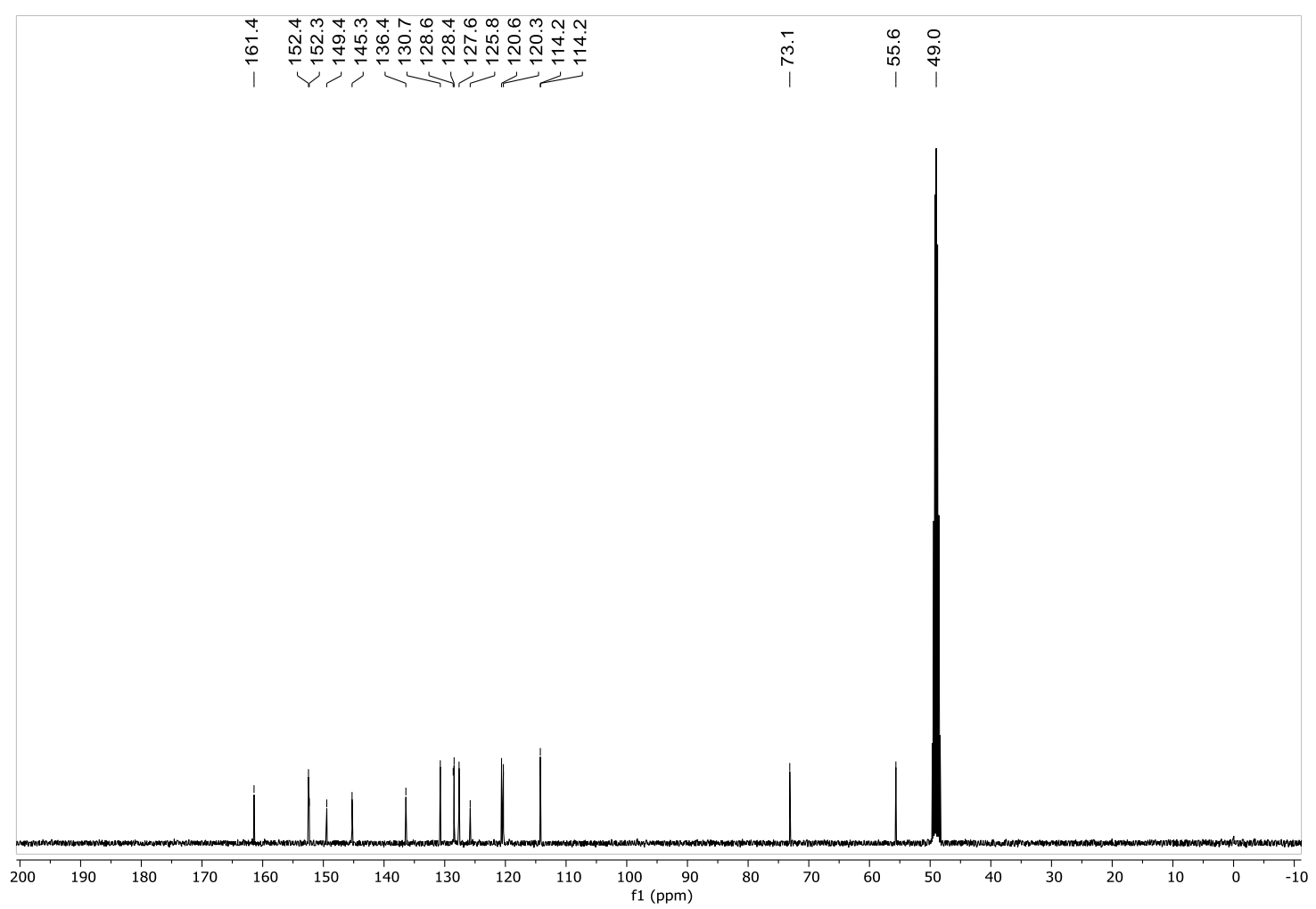


${ }^{1} \mathrm{H}$ NMR spectrum in DMSO- $\mathrm{d}_{6}$ of compound $9 \mathbf{q}$

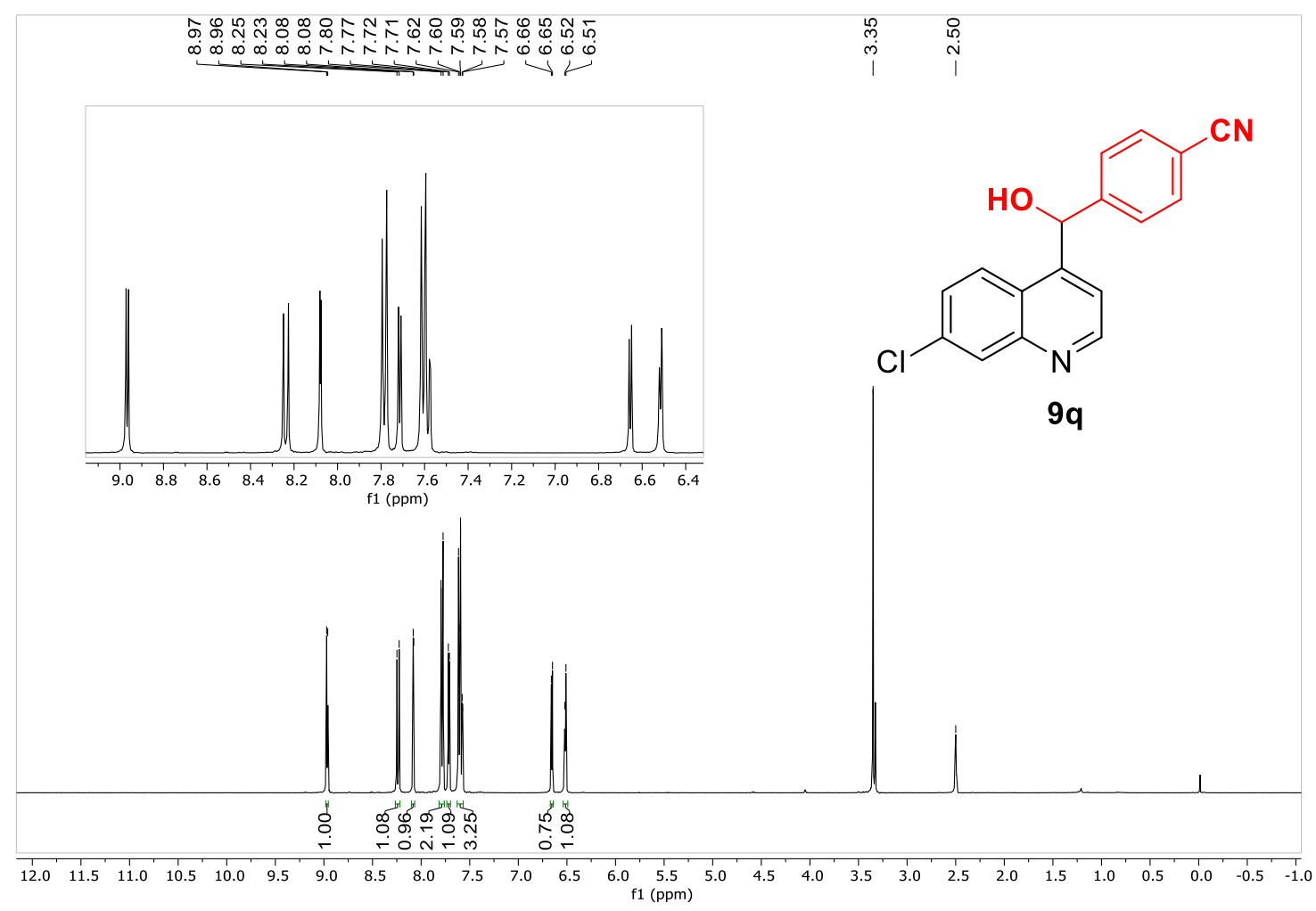

${ }^{13} \mathrm{C}\left\{{ }^{1} \mathrm{H}\right\}$ NMR spectrum in DMSO-d 6 of compound $\mathbf{9 q}$

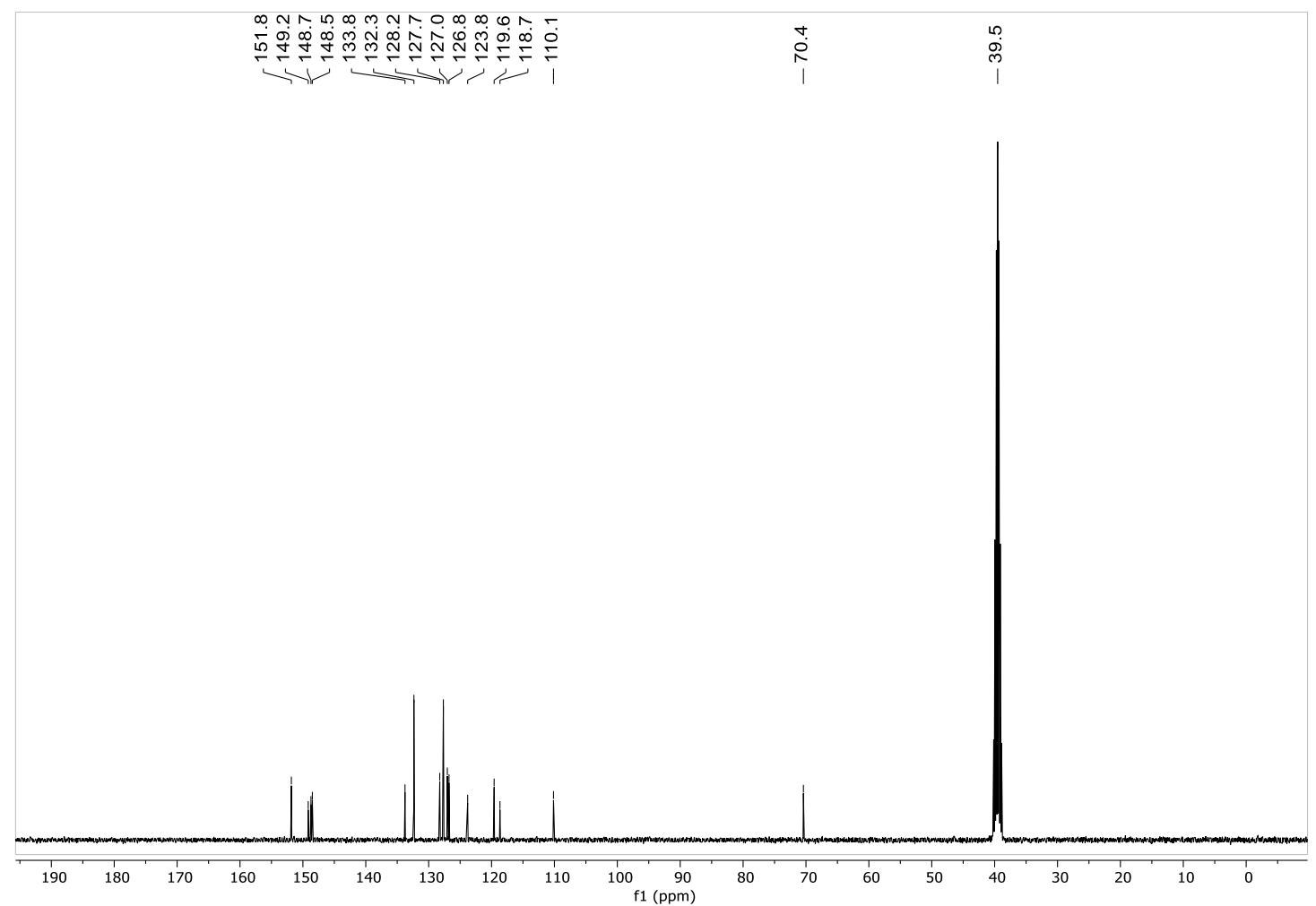


${ }^{1} \mathrm{H}$ NMR spectrum in DMSO- $\mathrm{d}_{6}$ of compound 9r

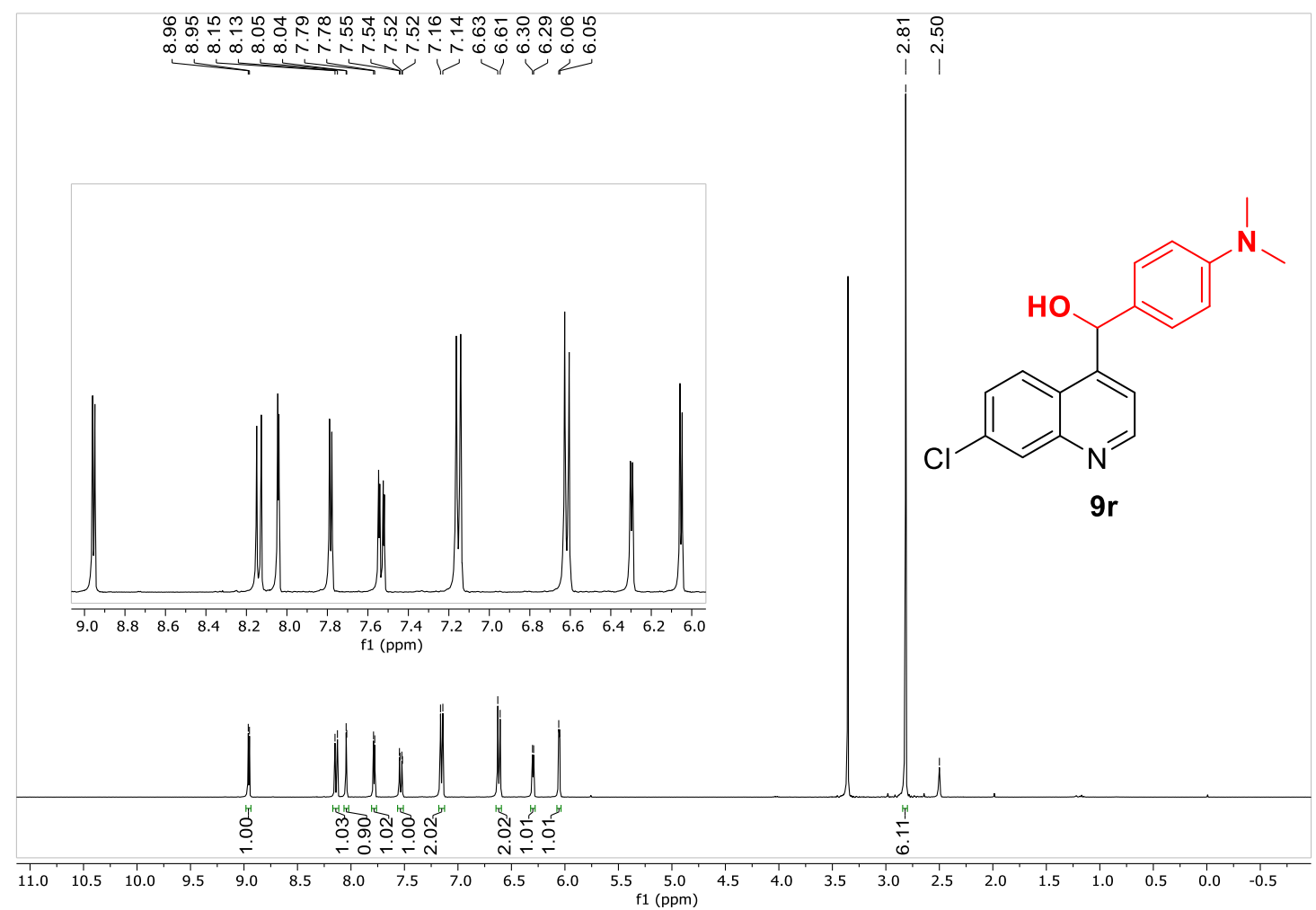

${ }^{13} \mathrm{C}\left\{{ }^{1} \mathrm{H}\right\}$ NMR spectrum in DMSO-d 6 of compound $9 \mathbf{r}$

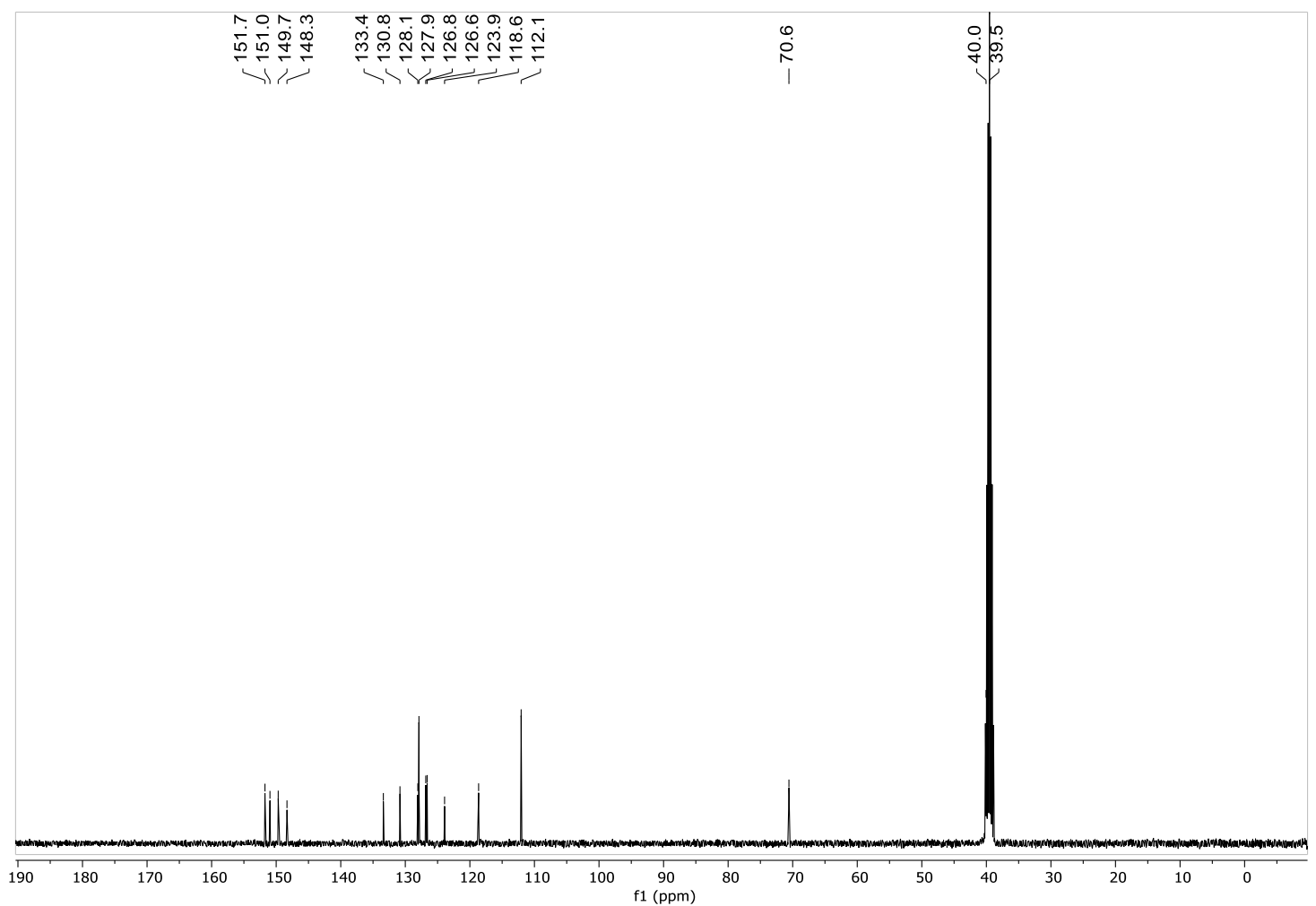


${ }^{1} \mathrm{H}$ NMR spectrum in DMSO- $\mathrm{d}_{6}$ of compound 9s

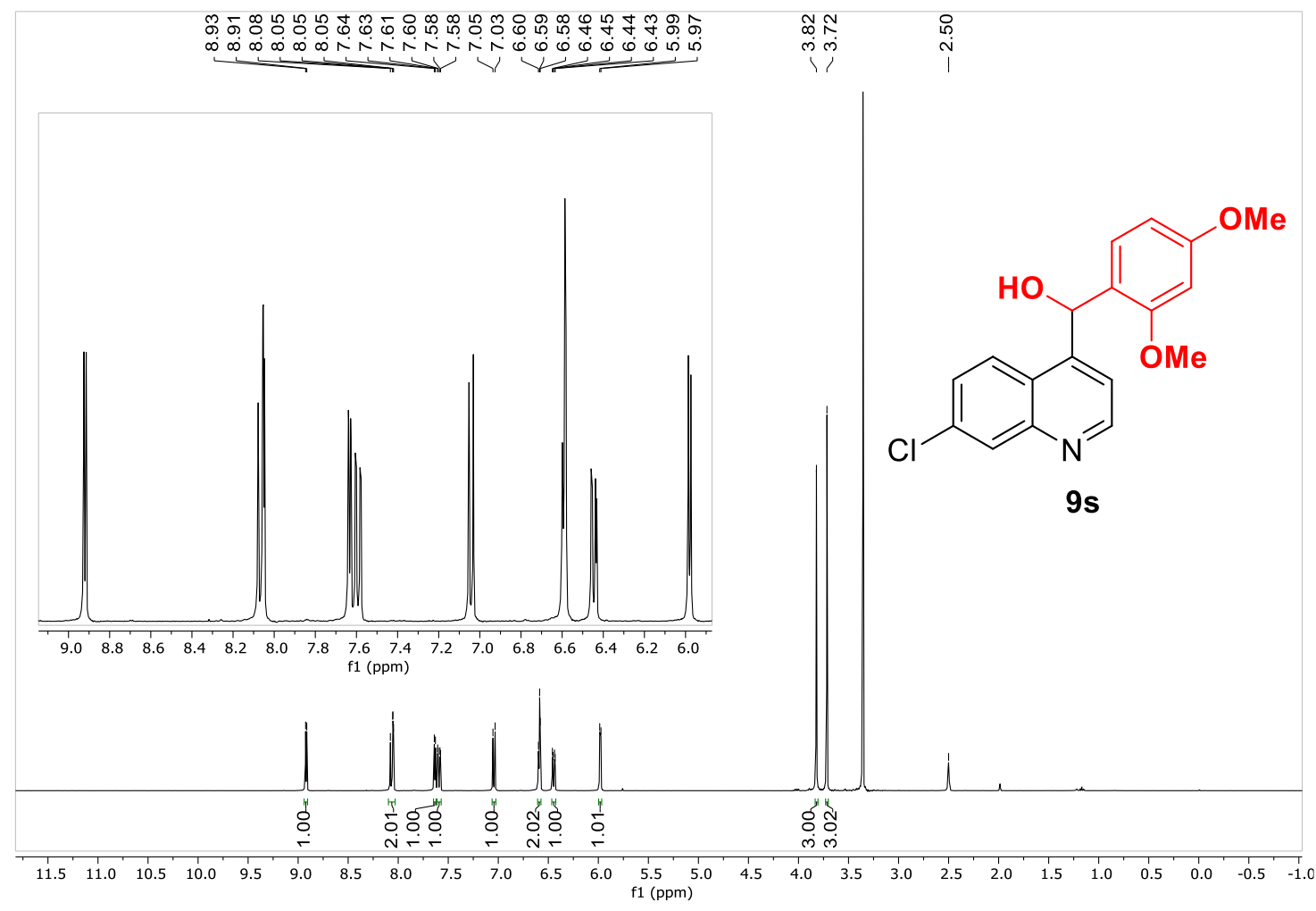

${ }^{13} \mathrm{C}\left\{{ }^{1} \mathrm{H}\right\}$ NMR spectrum in DMSO-d $\mathrm{d}_{6}$ of compound 9s

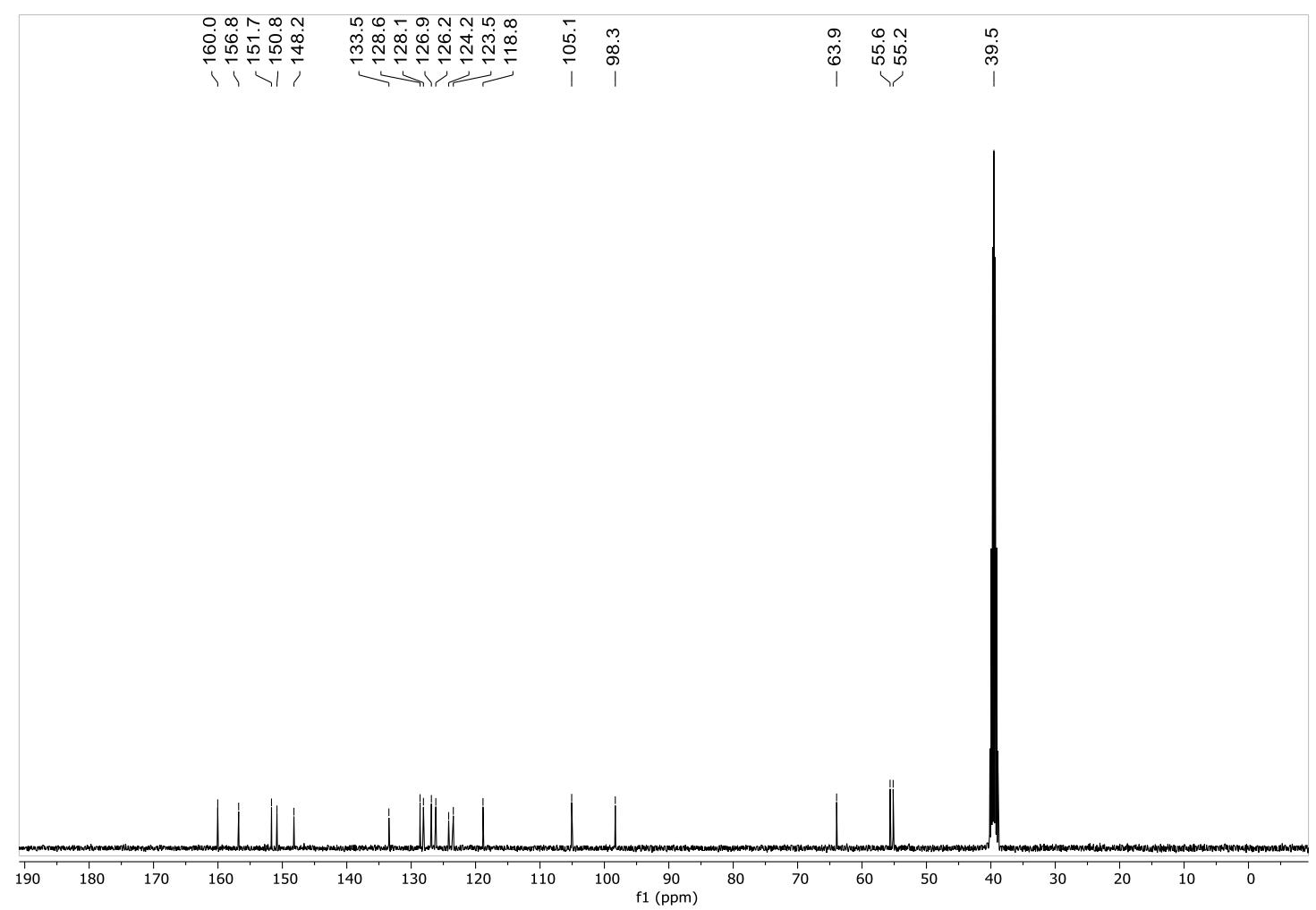


${ }^{1} \mathrm{H}$ NMR spectrum in DMSO- $\mathrm{d}_{6}$ of compound $9 \mathbf{t}$

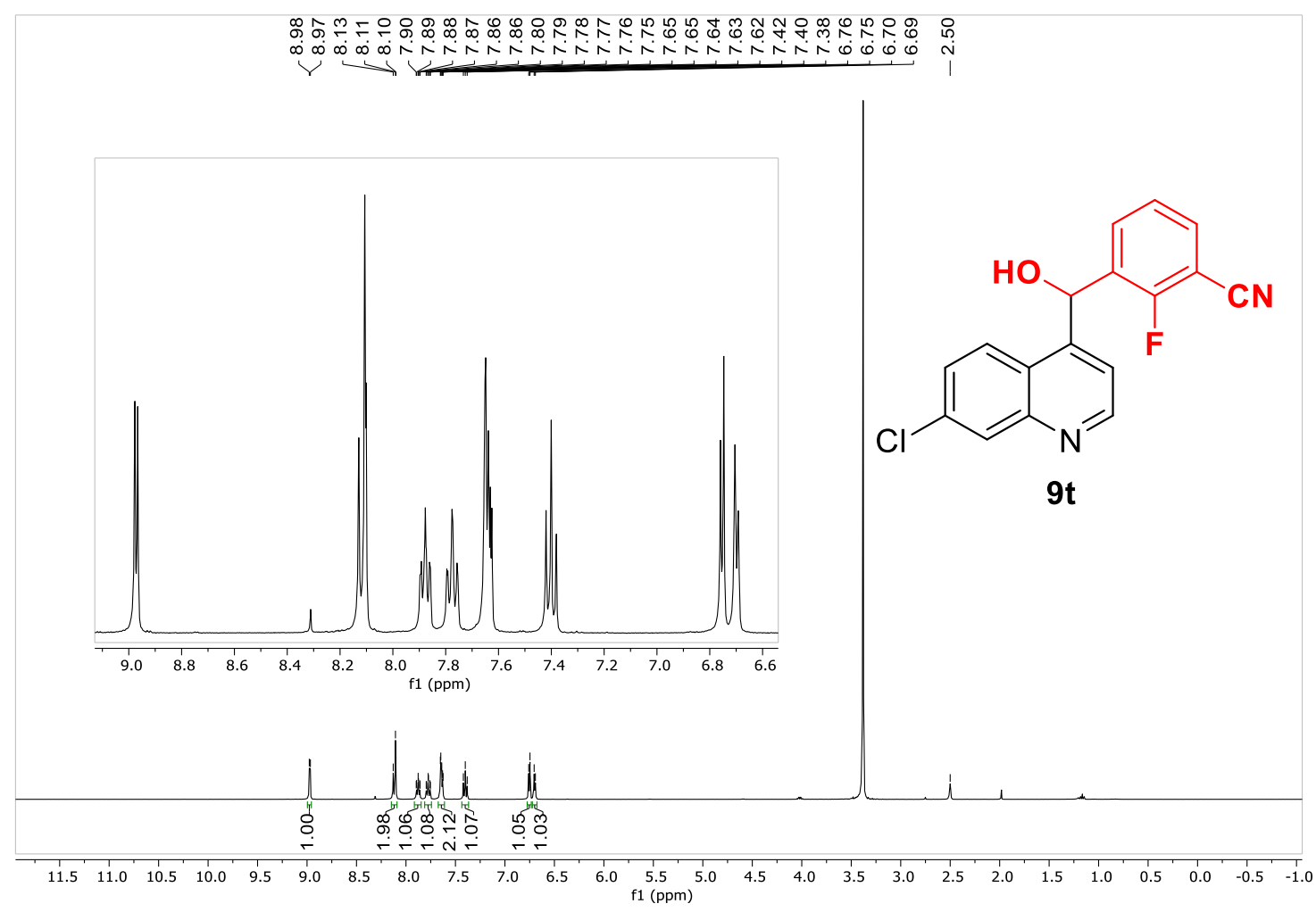

${ }^{13} \mathrm{C}\left\{{ }^{1} \mathrm{H}\right\}$ NMR spectrum in DMSO-d $\mathrm{d}_{6}$ of compound $9 \mathrm{t}$

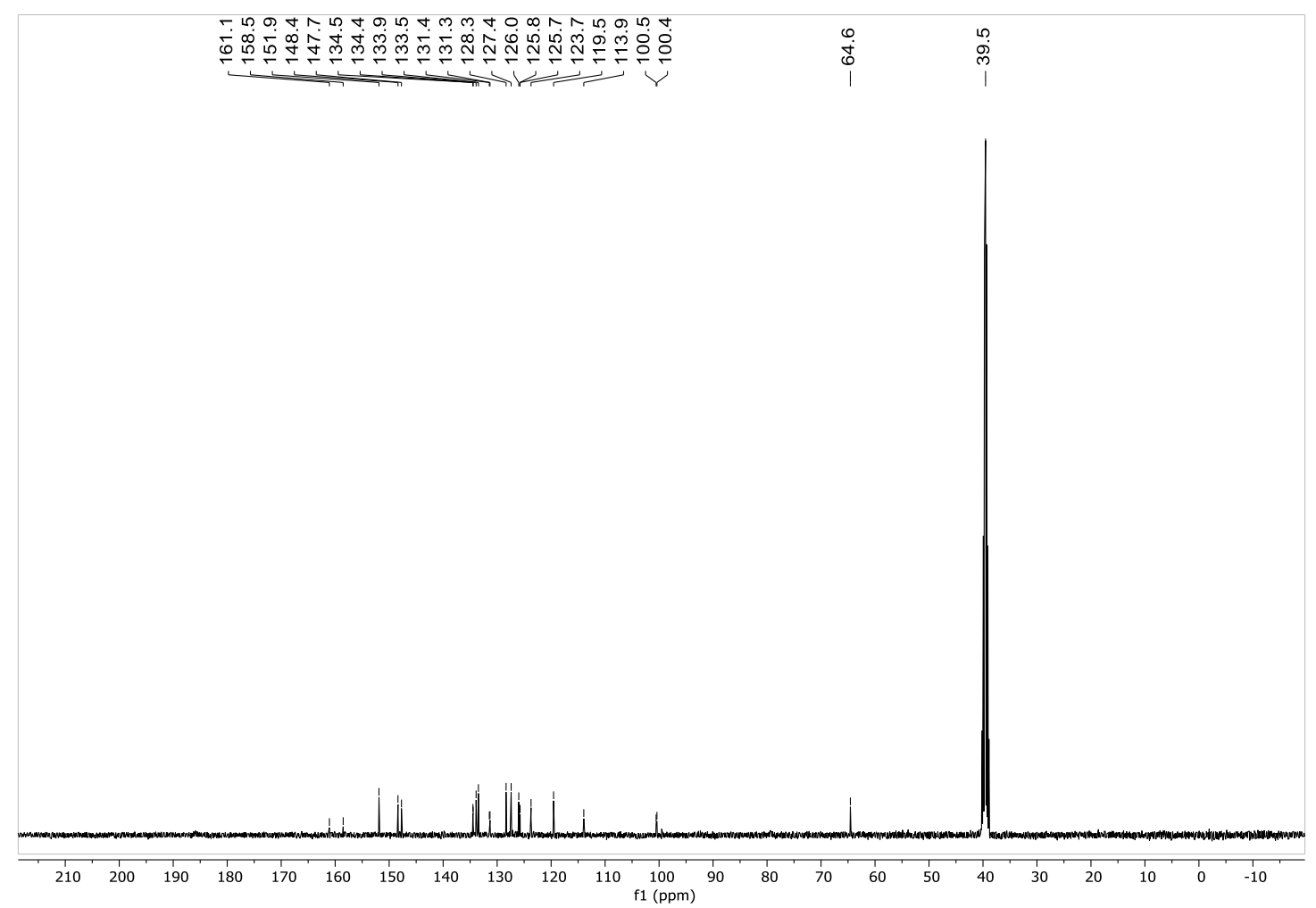


${ }^{1} \mathrm{H}$ NMR spectrum in DMSO- $\mathrm{d}_{6}$ of compound $9 \mathbf{u}$

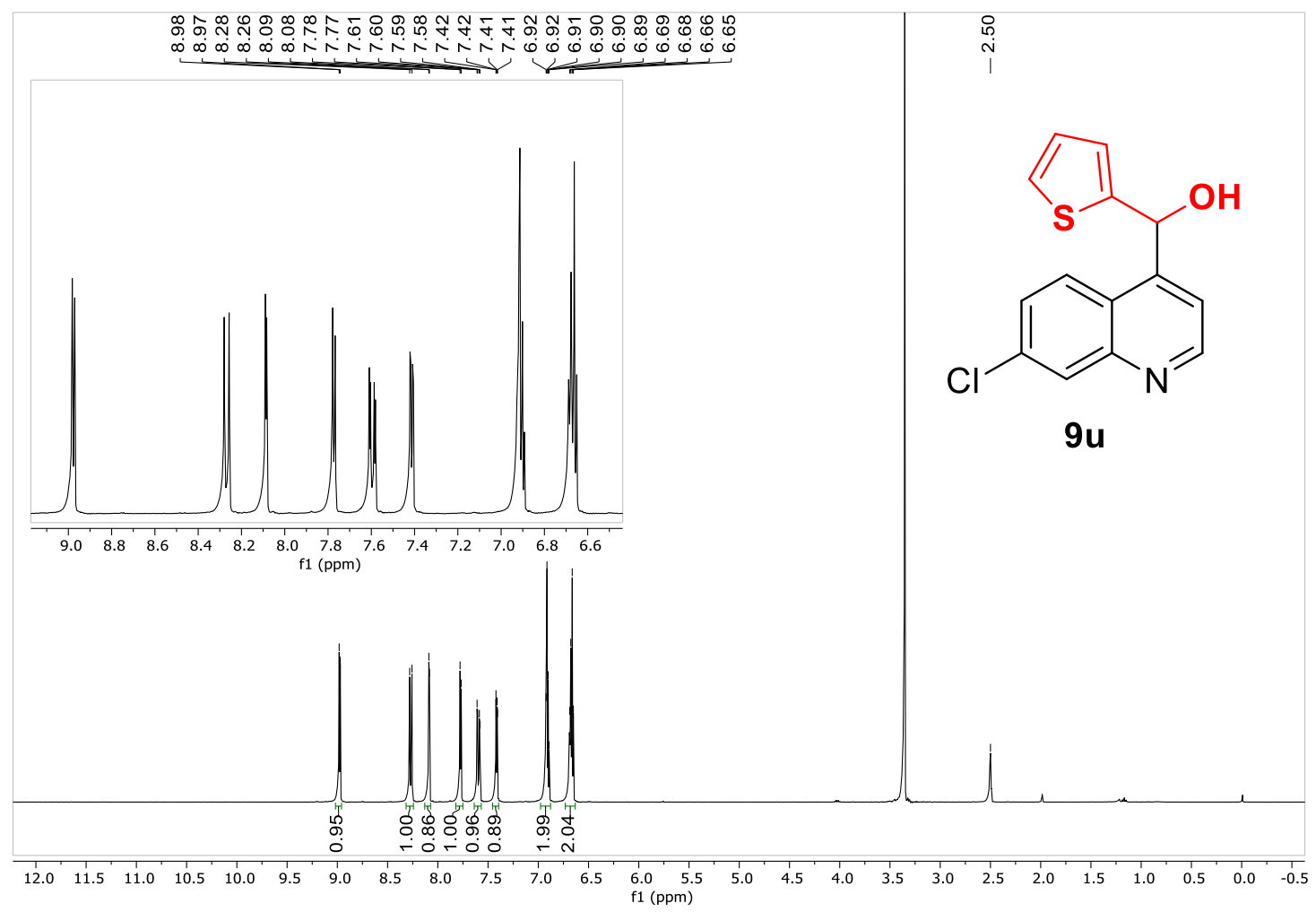

${ }^{13} \mathrm{C}\left\{{ }^{1} \mathrm{H}\right\}$ NMR spectrum in DMSO-d 6 of compound $9 \mathbf{u}$

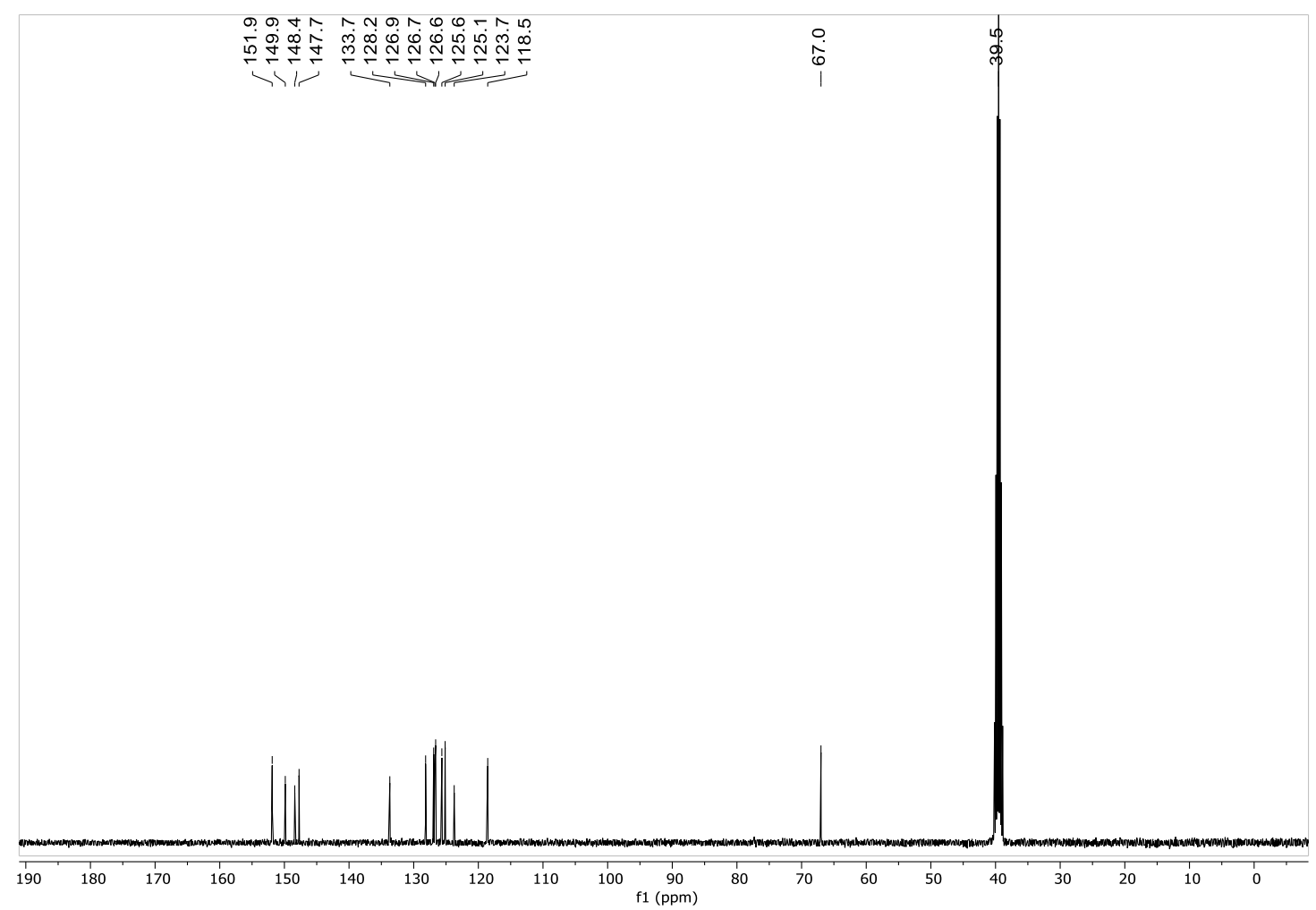


${ }^{1} \mathrm{H}$ NMR spectrum in DMSO-d $\mathrm{d}_{6}$ of compound 9v

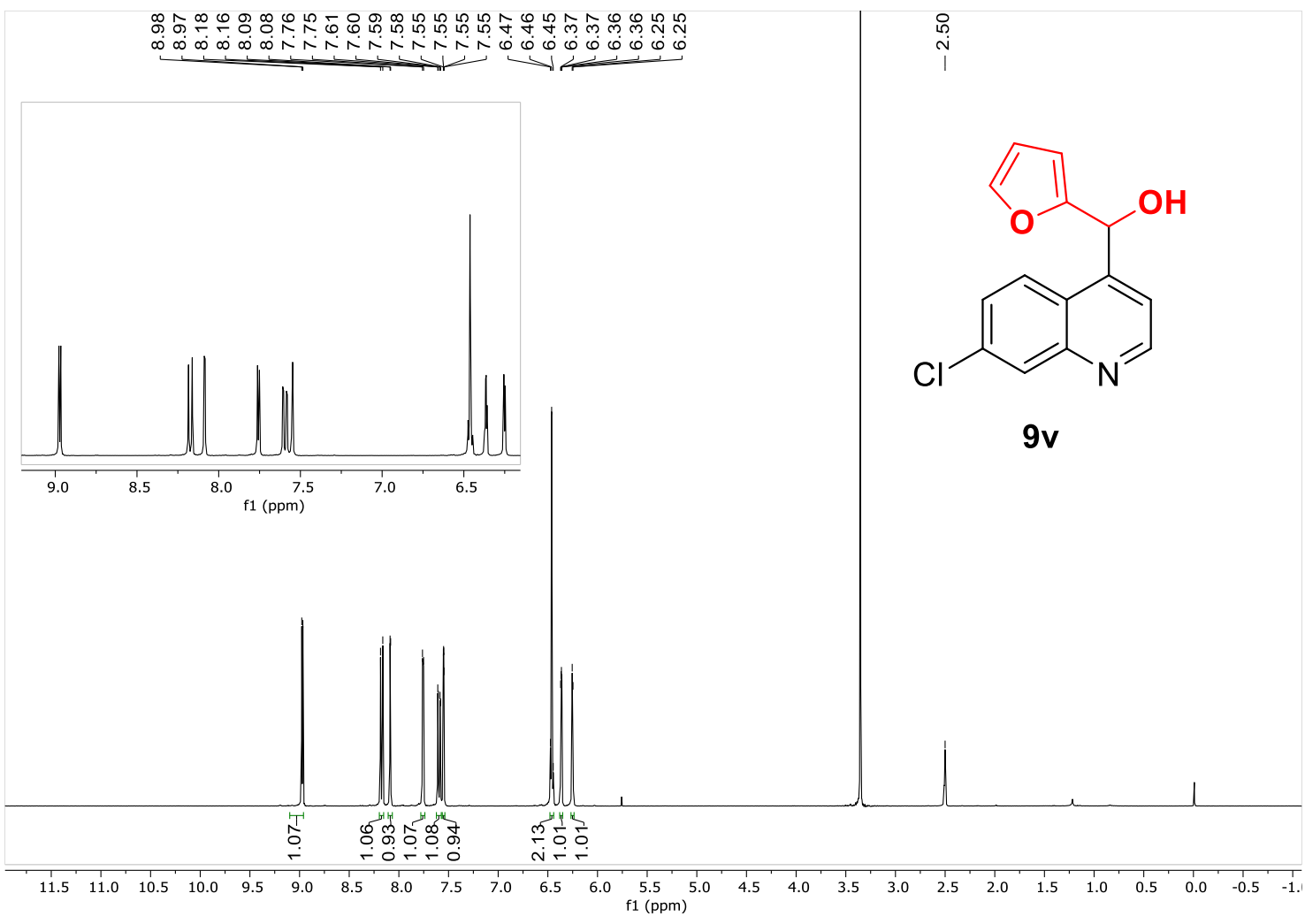

${ }^{13} \mathrm{C}\left\{{ }^{1} \mathrm{H}\right\}$ NMR spectrum in DMSO-d $\mathrm{d}_{6}$ of compound 9v

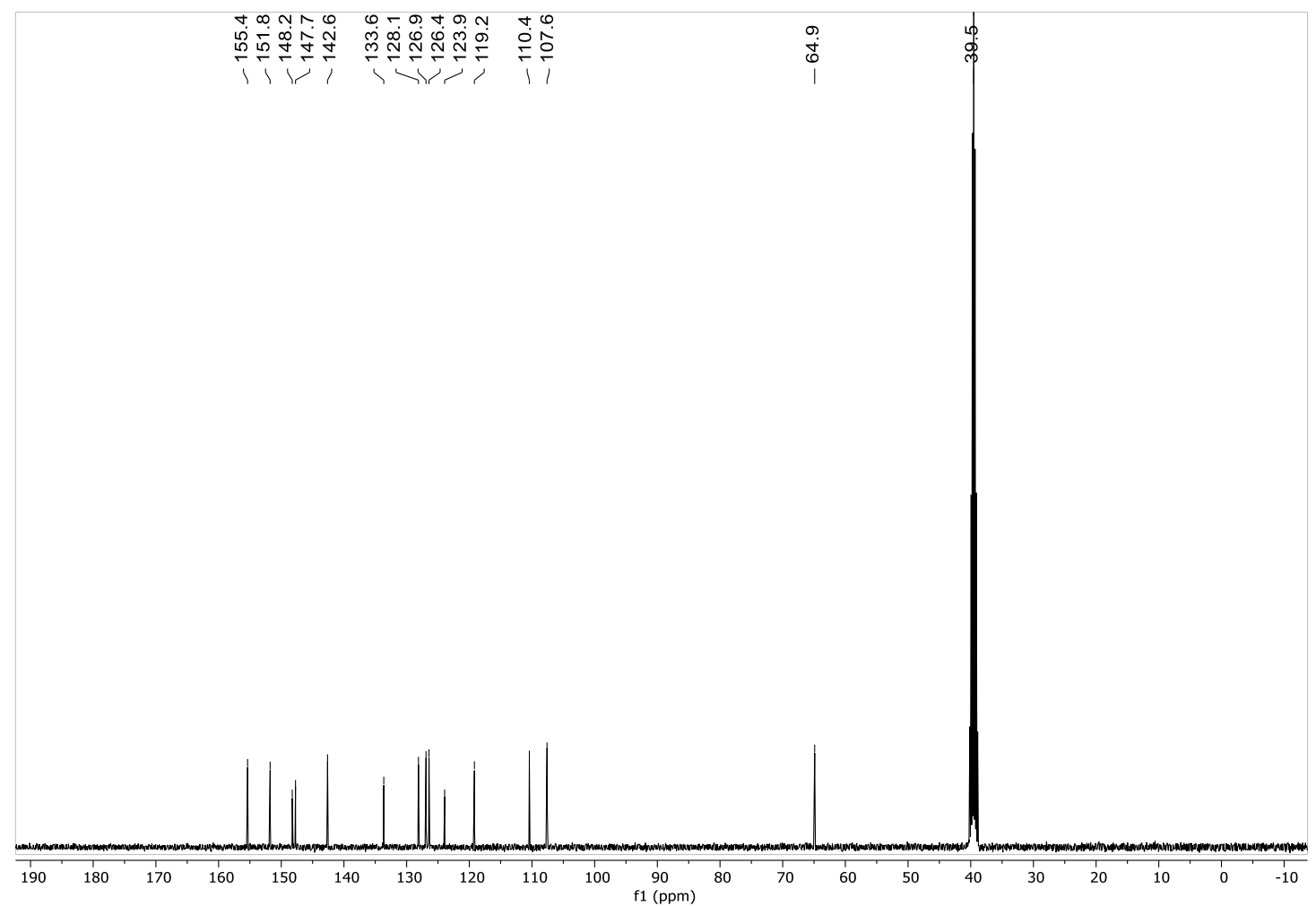


${ }^{1} \mathrm{H}$ NMR spectrum in DMSO- $\mathrm{d}_{6}$ of compound $\mathbf{9 w}$

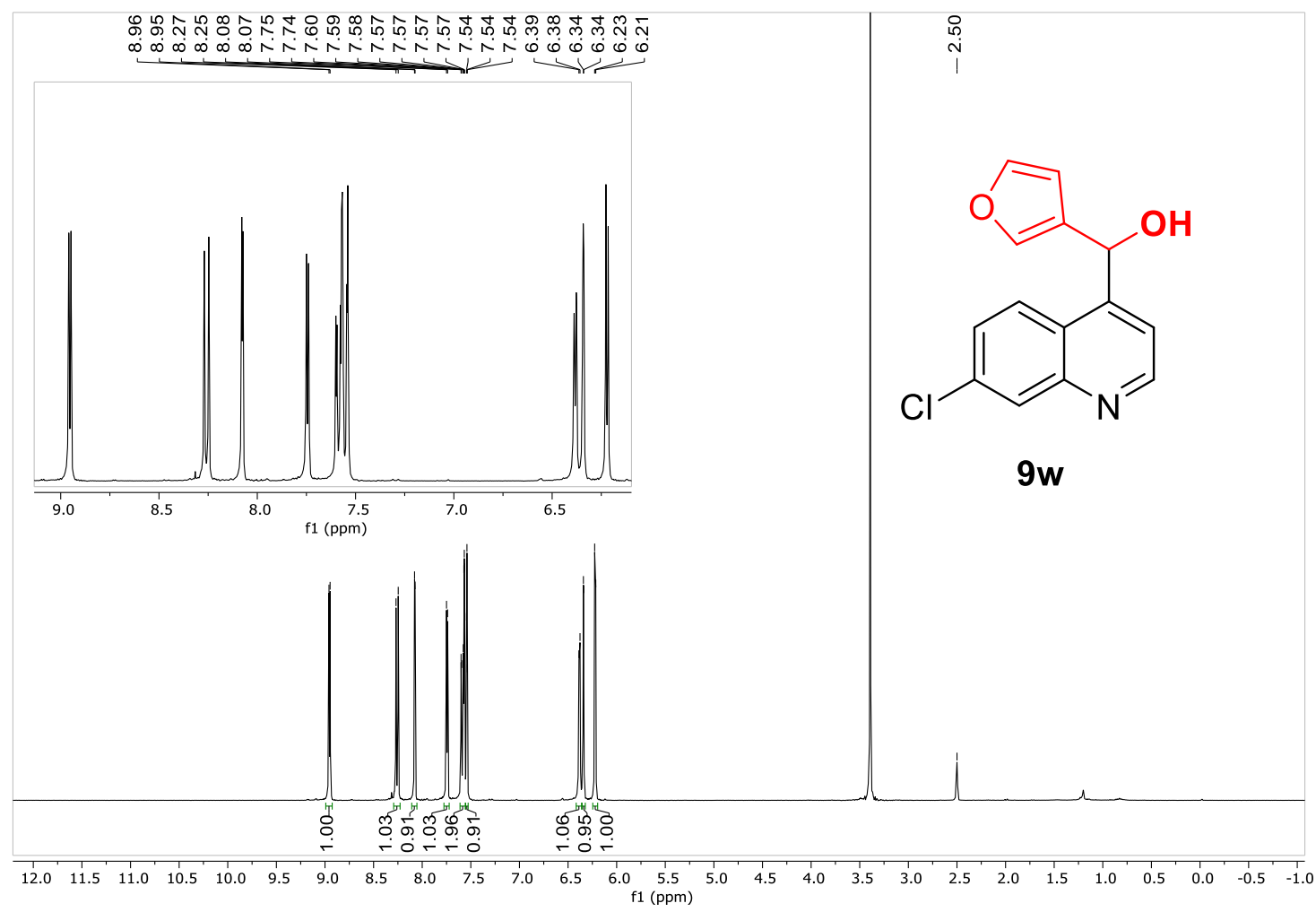

${ }^{13} \mathrm{C}\left\{{ }^{1} \mathrm{H}\right\}$ NMR spectrum in DMSO- $\mathrm{d}_{6}$ of compound $\mathbf{9 w}_{\mathbf{w}}$

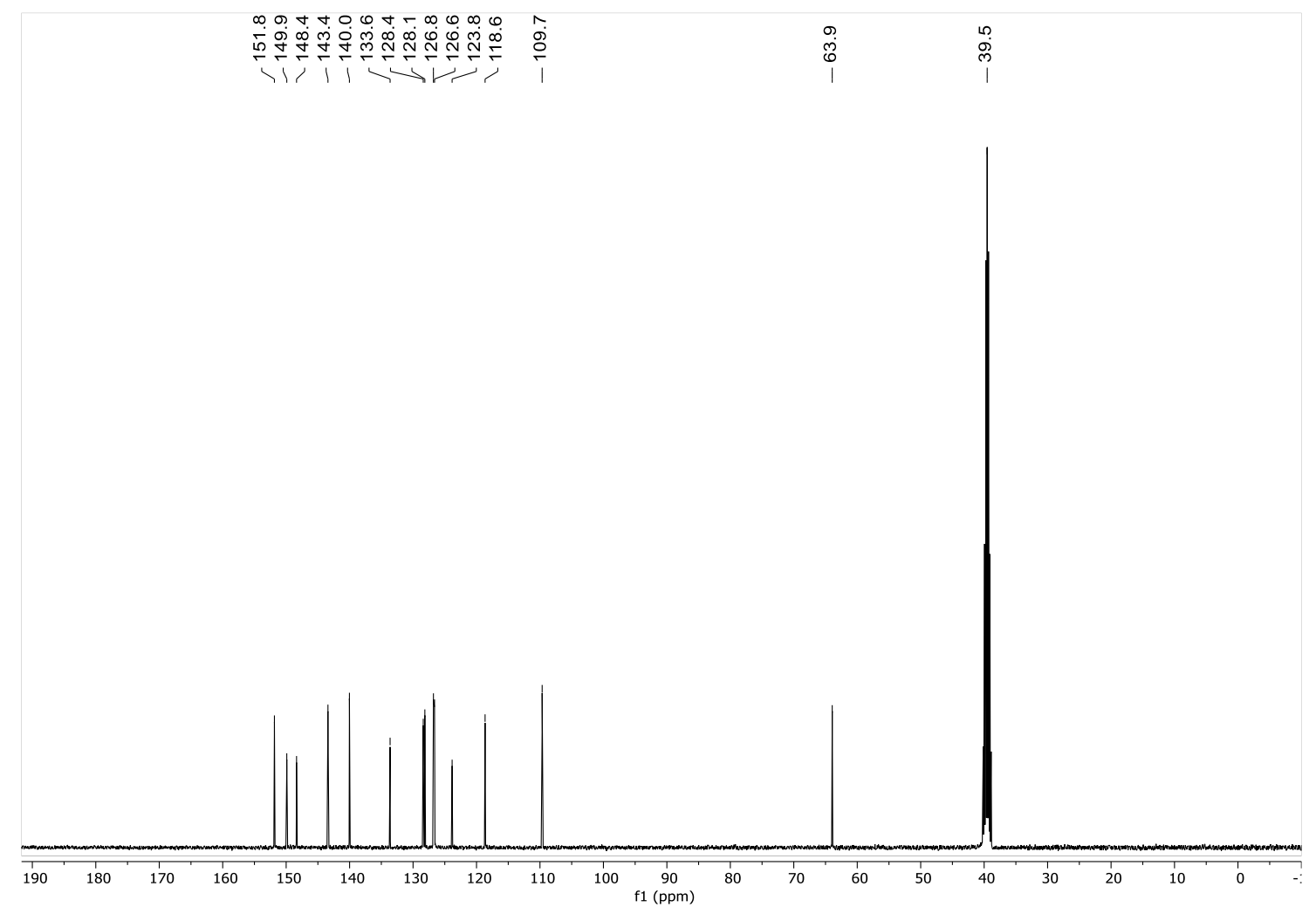


${ }^{1} \mathrm{H}$ NMR spectrum in $\mathrm{CDCl}_{3}$ of compound $\mathbf{9 x}$

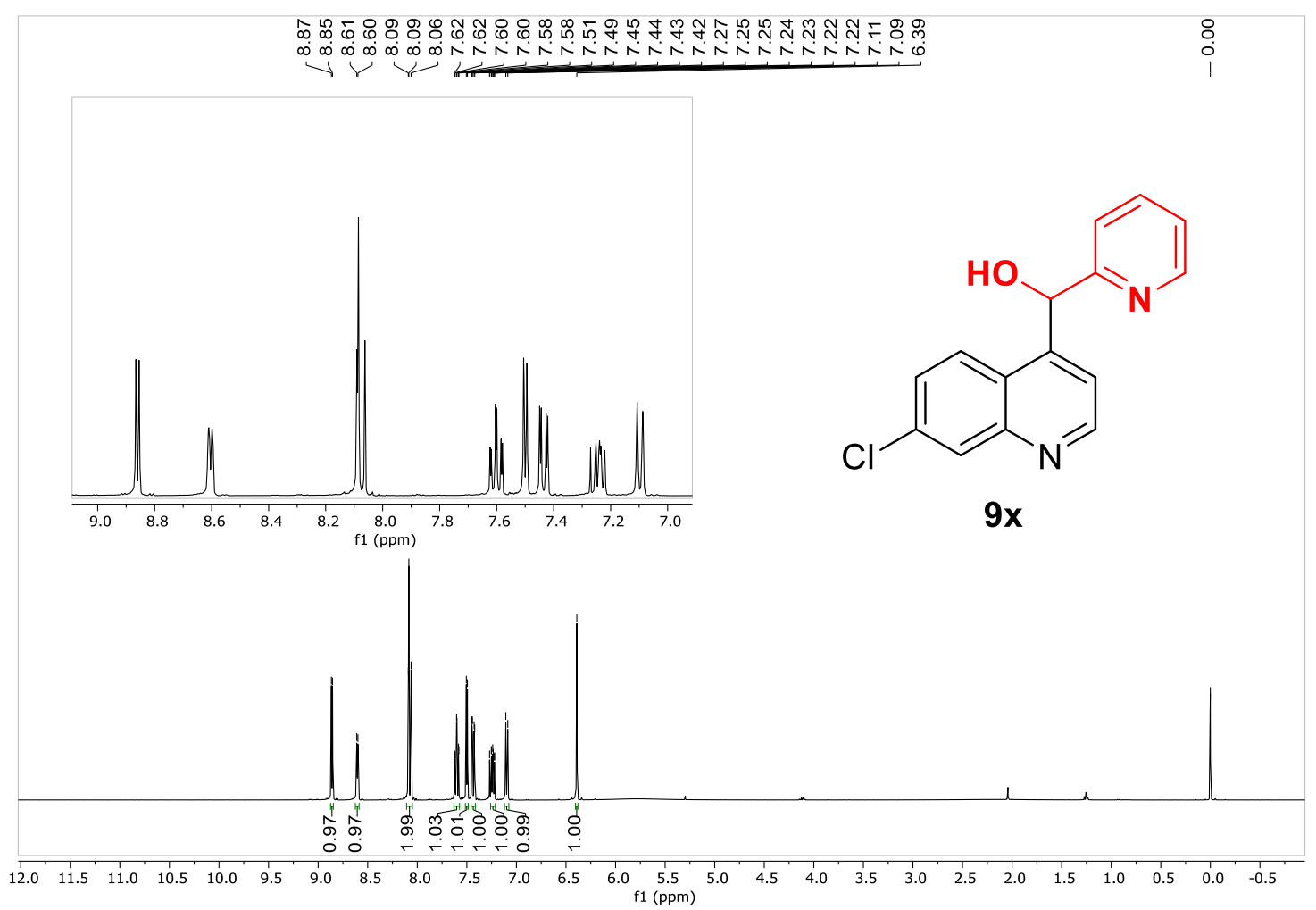

${ }^{13} \mathrm{C}\left\{{ }^{1} \mathrm{H}\right\}$ NMR spectrum in $\mathrm{CDCl}_{3}$ of compound $\mathbf{9 x}$

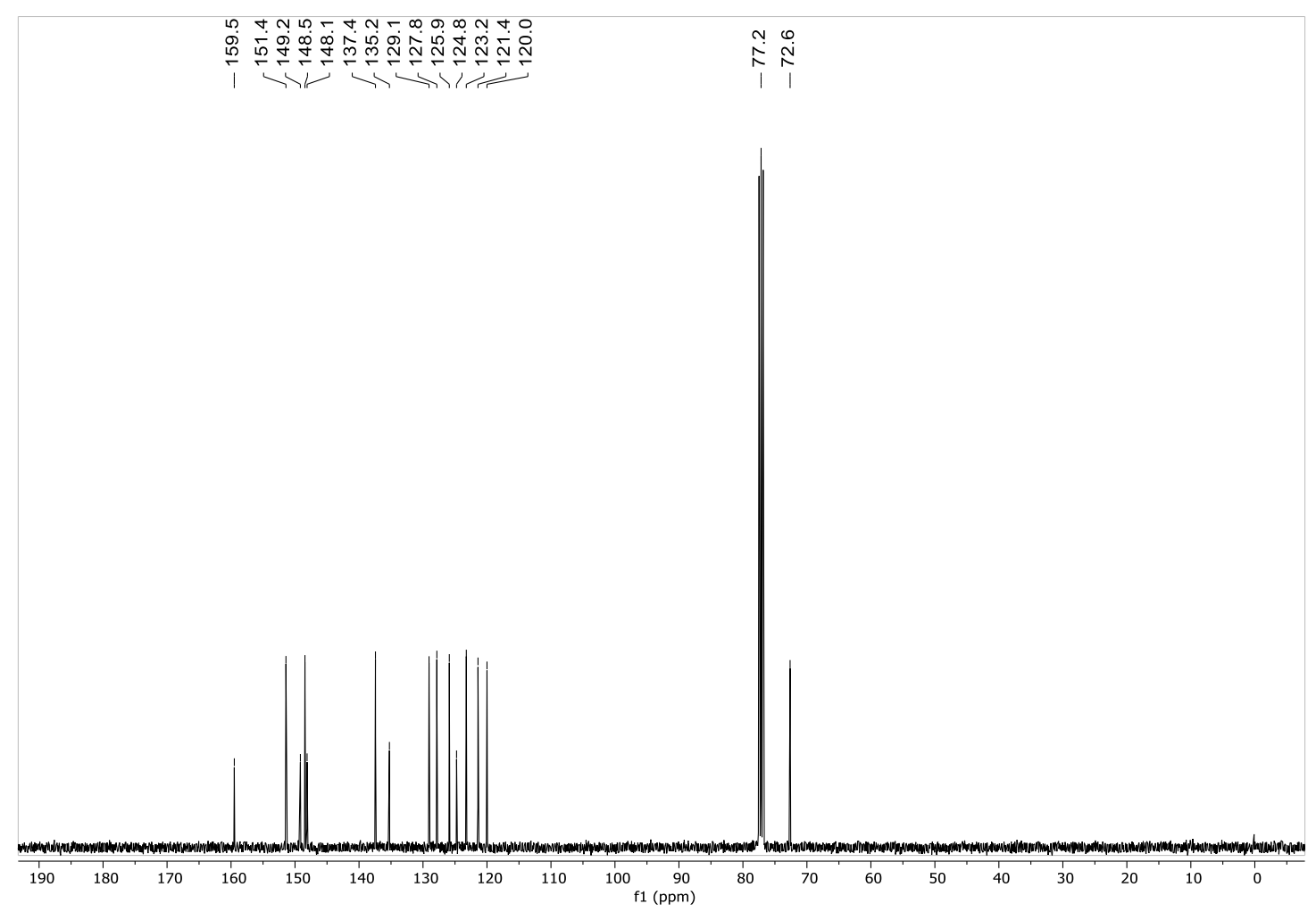


${ }^{1} \mathrm{H}$ NMR spectrum in DMSO- $\mathrm{d}_{6}$ of compound $9 \mathbf{y}$

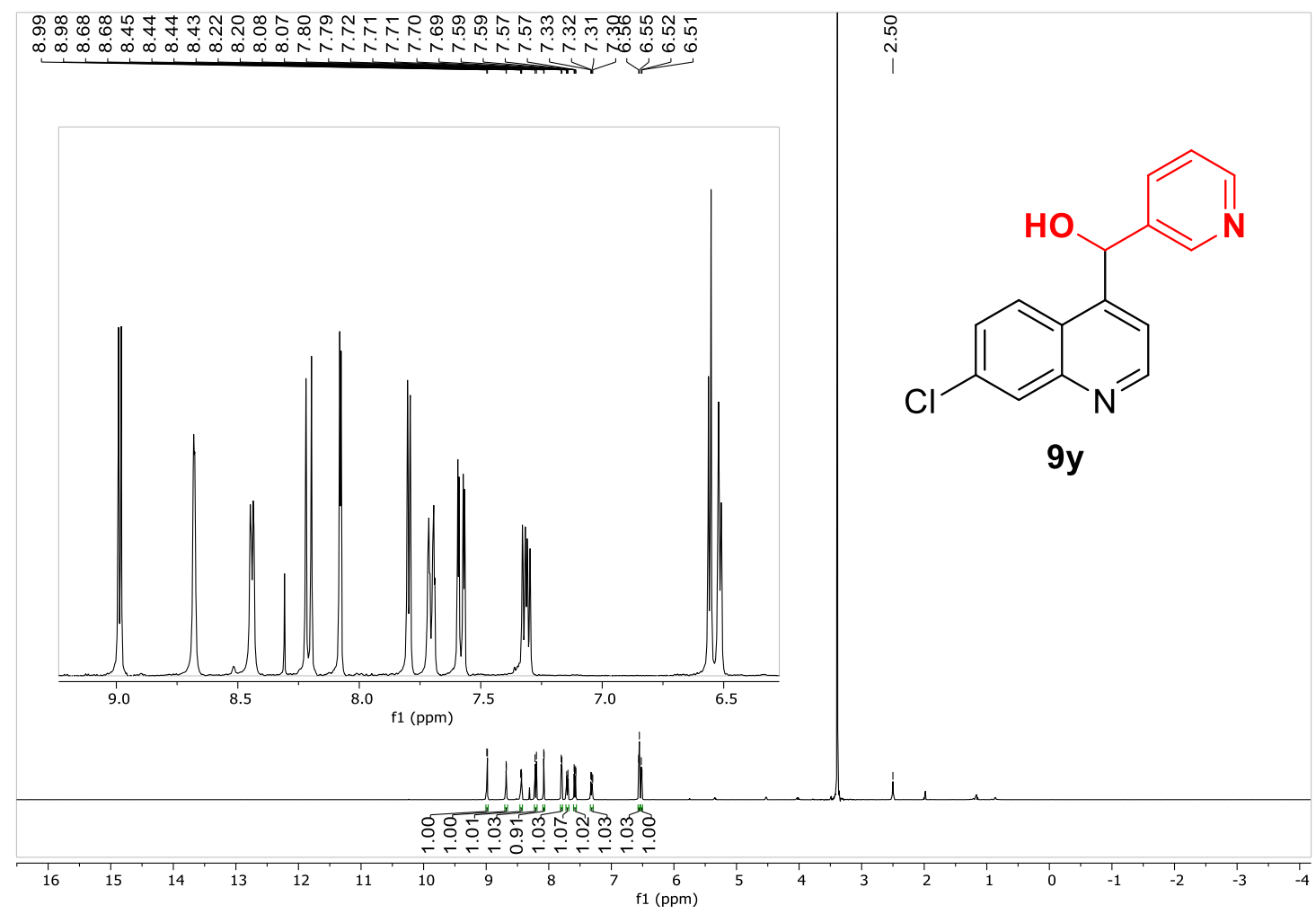

${ }^{13} \mathrm{C}\left\{{ }^{1} \mathrm{H}\right\}$ NMR spectrum in DMSO-d $\mathrm{d}_{6}$ of compound $9 \mathbf{y}$

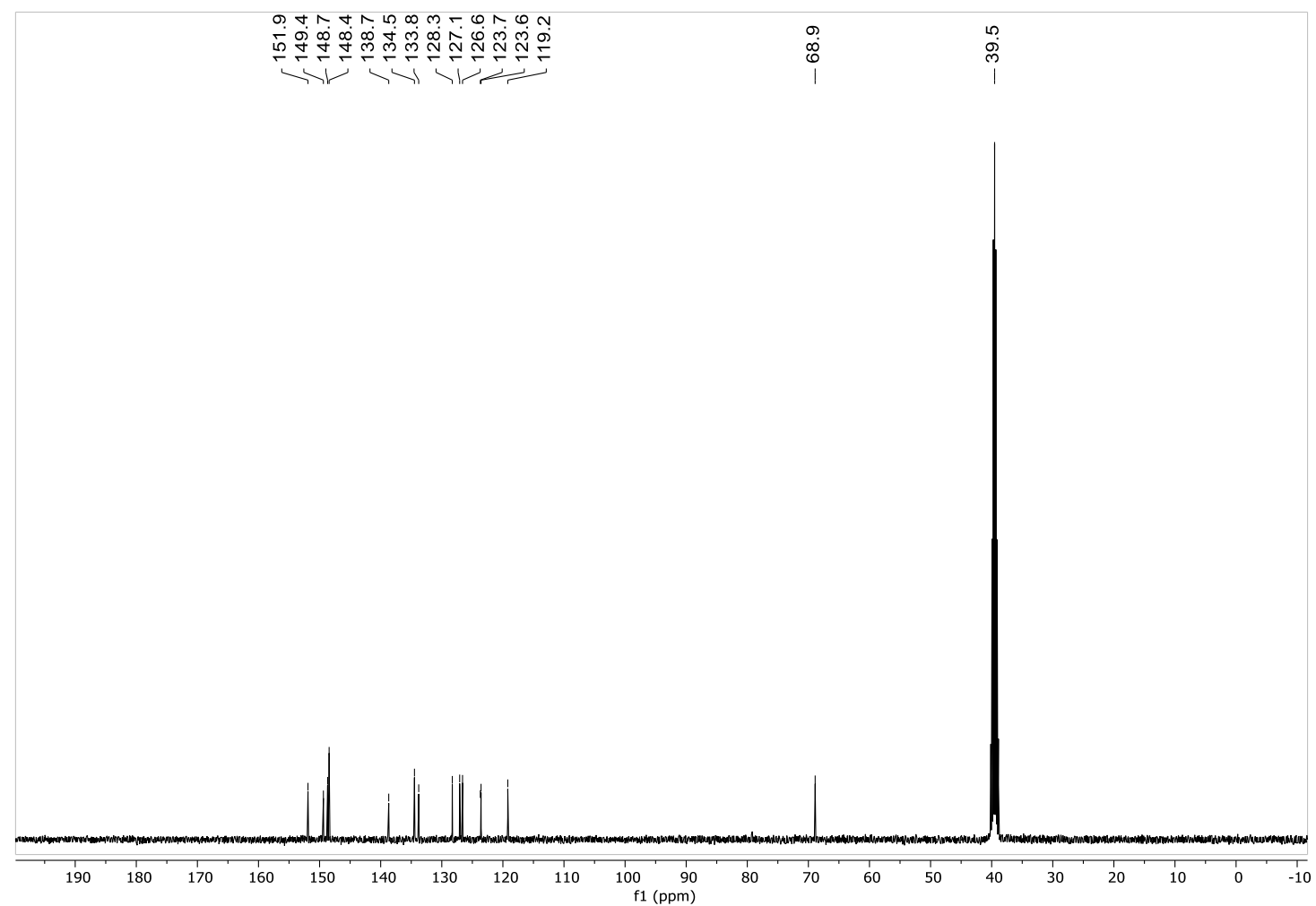


${ }^{1} \mathrm{H}$ NMR spectrum in $\mathrm{CDCl}_{3}$ of compound $\mathbf{9 z}$

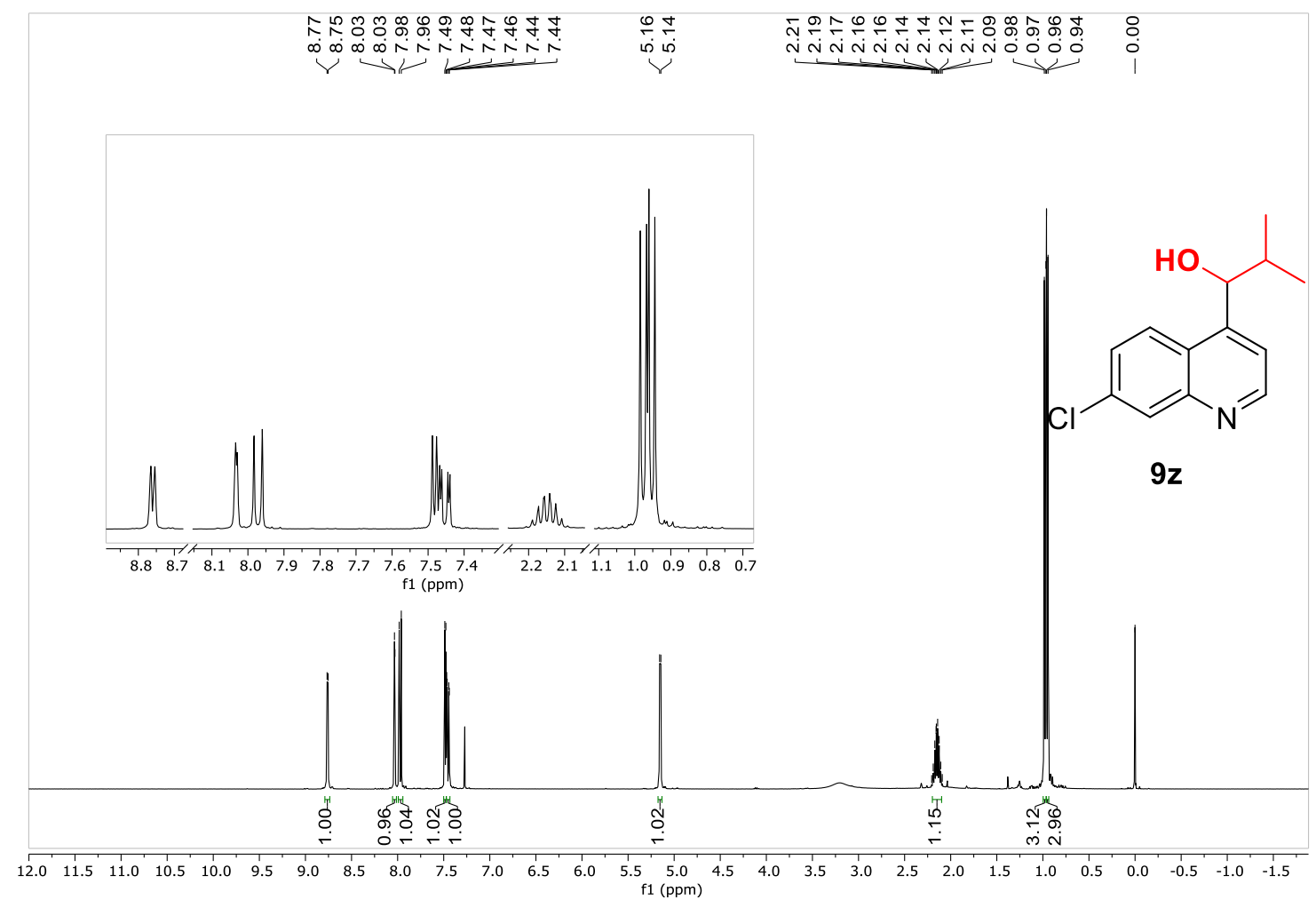

${ }^{13} \mathrm{C}\left\{{ }^{1} \mathrm{H}\right\}$ NMR spectrum in $\mathrm{CDCl}_{3}$ of compound $\mathbf{9 z}$

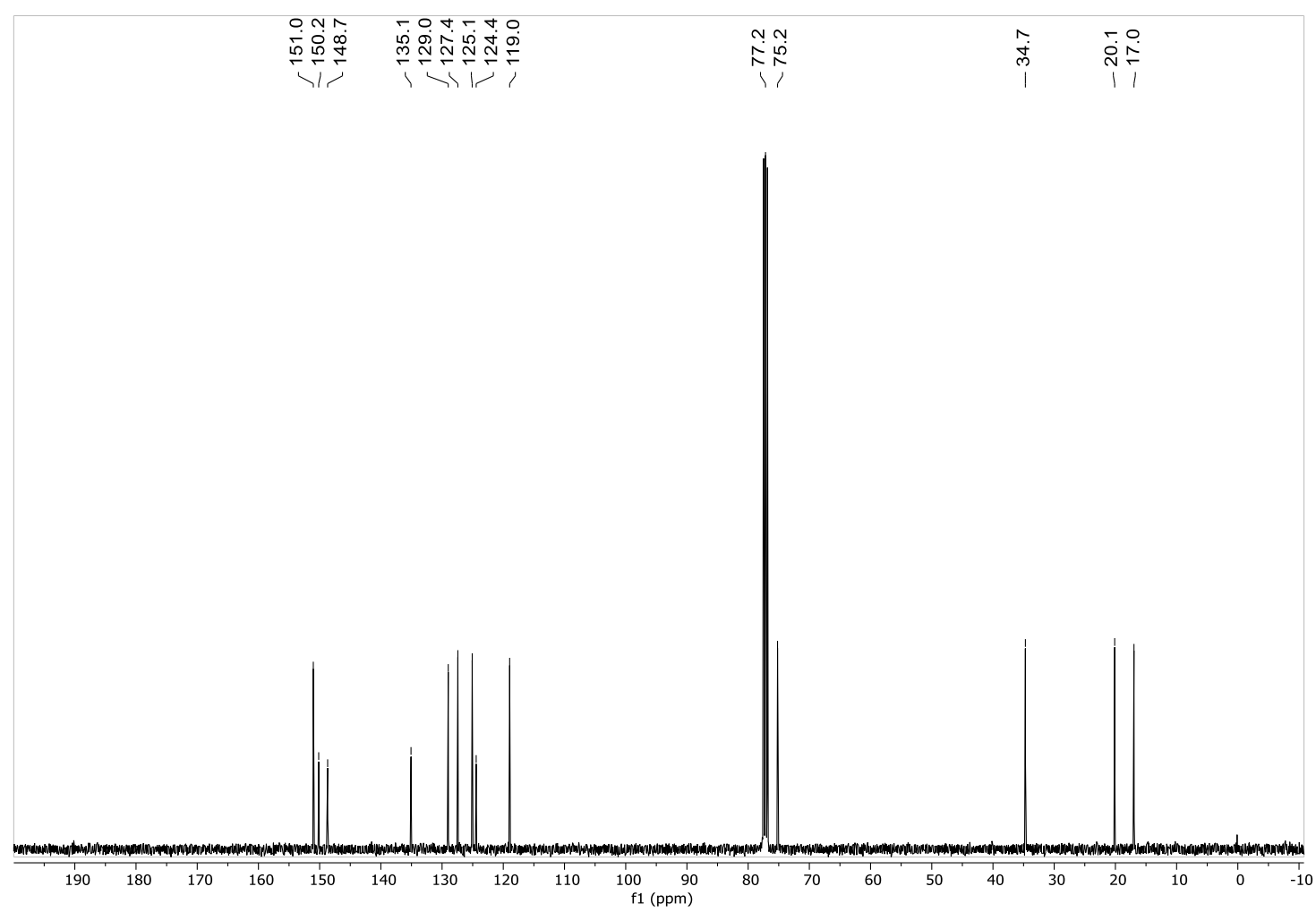


${ }^{1} \mathrm{H}$ NMR spectrum in DMSO- $\mathrm{d}_{6}$ of compound 9aa

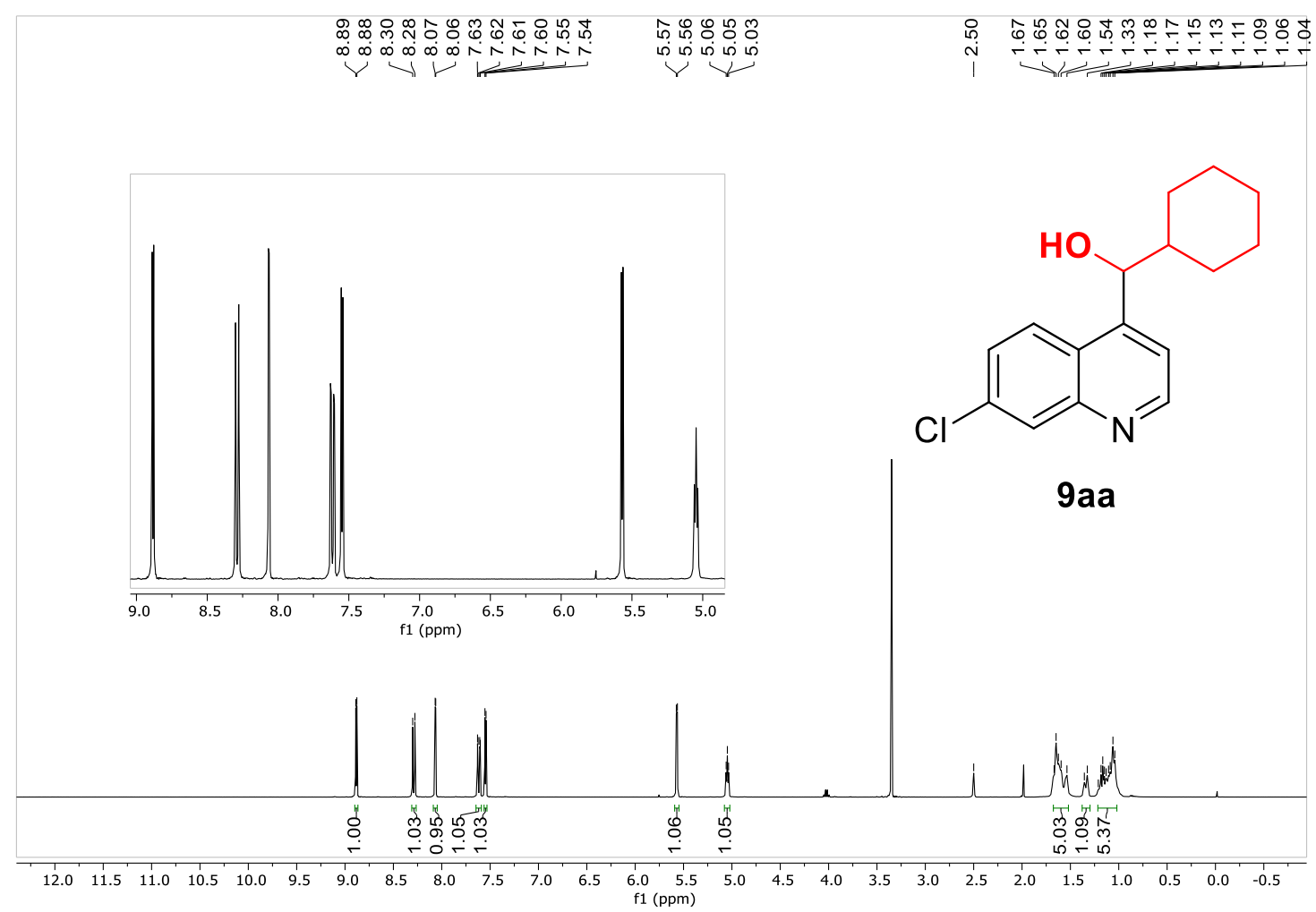

${ }^{13} \mathrm{C}\left\{{ }^{1} \mathrm{H}\right\}$ NMR spectrum in DMSO-d 6 of compound 9aa

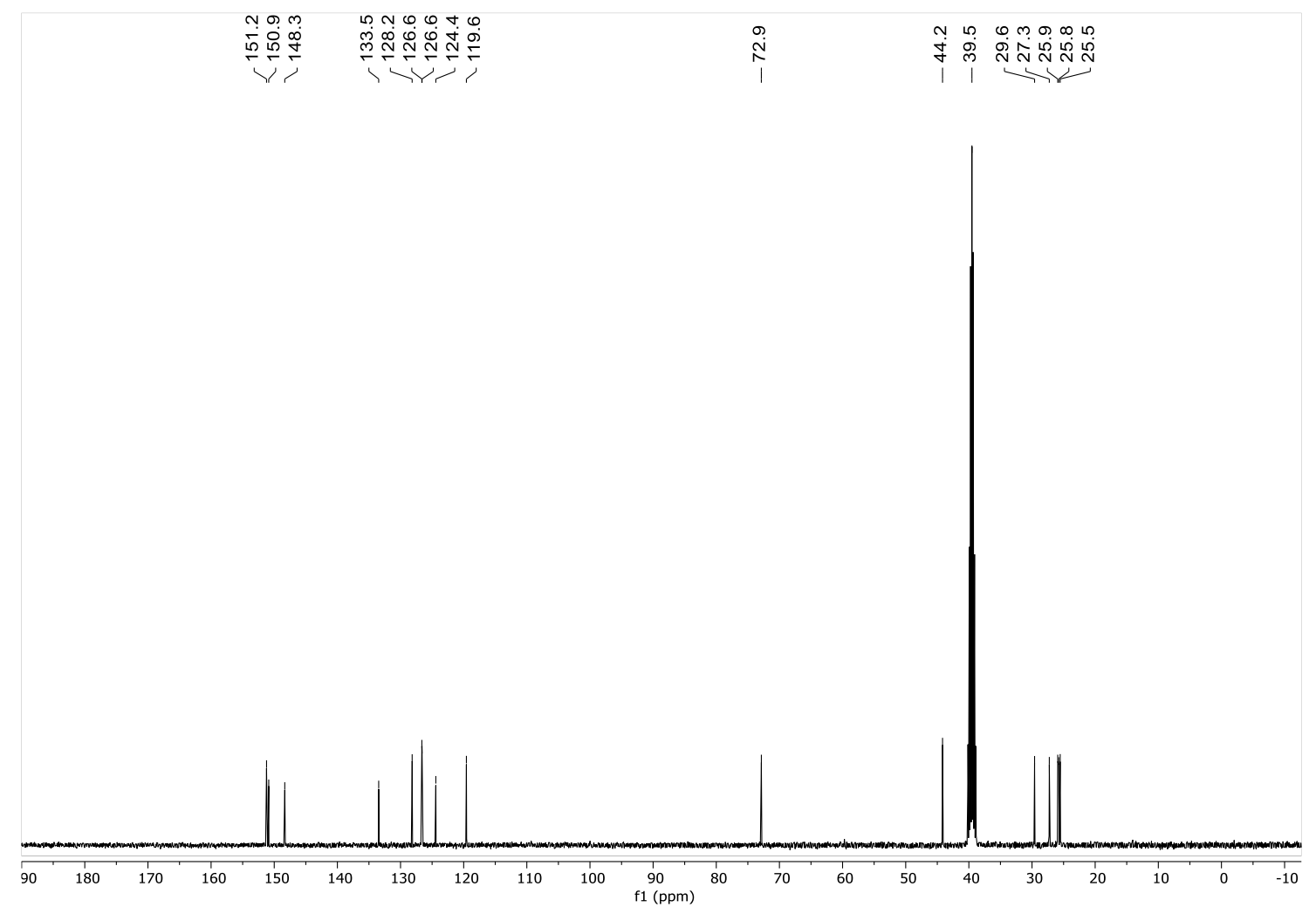


${ }^{1} \mathrm{H}$ NMR spectrum in $\mathrm{CDCl}_{3}$ of compound 10a

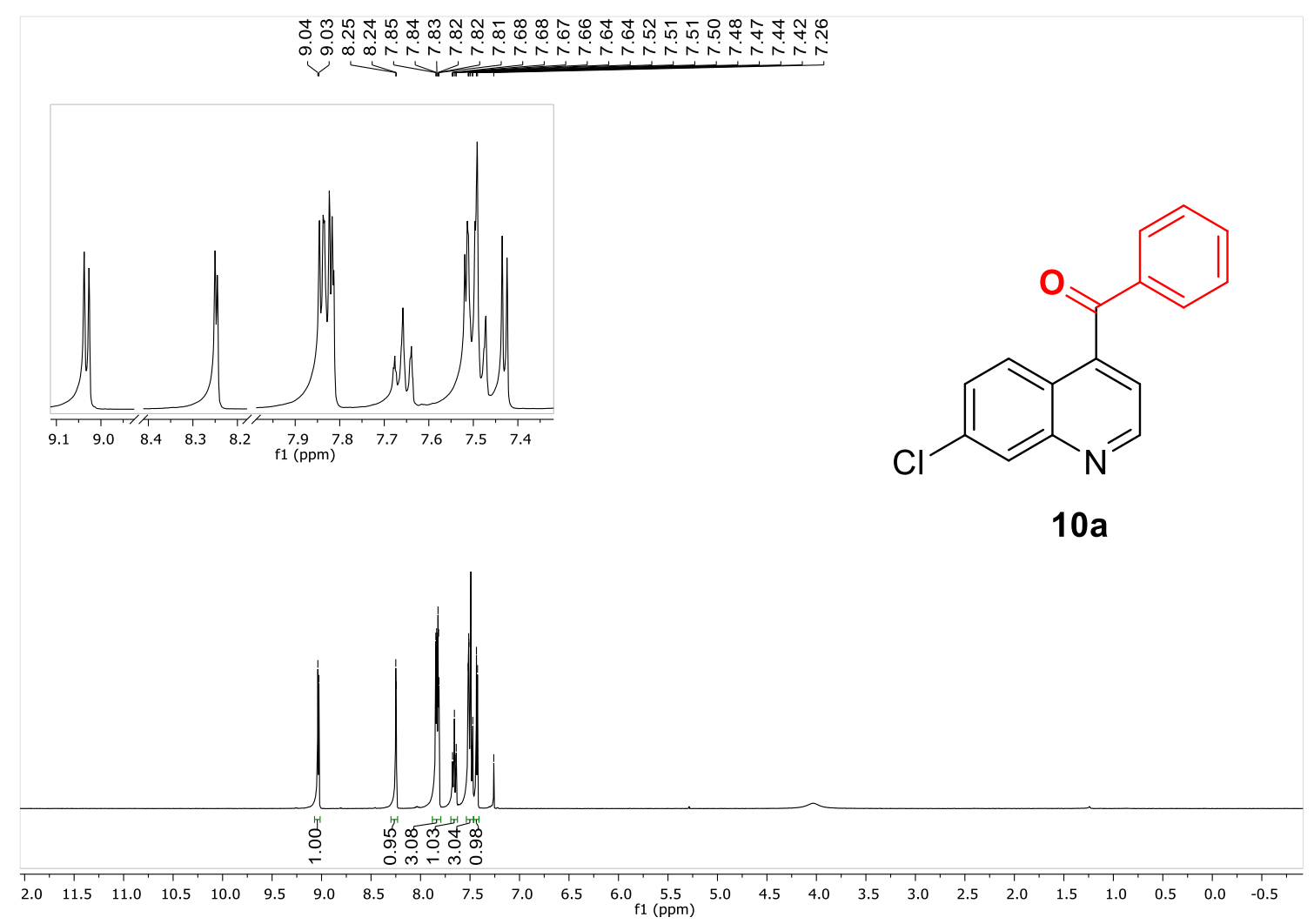

${ }^{13} \mathrm{C}\left\{{ }^{1} \mathrm{H}\right\}$ NMR spectrum in $\mathrm{CDCl}_{3}$ of compound 10a

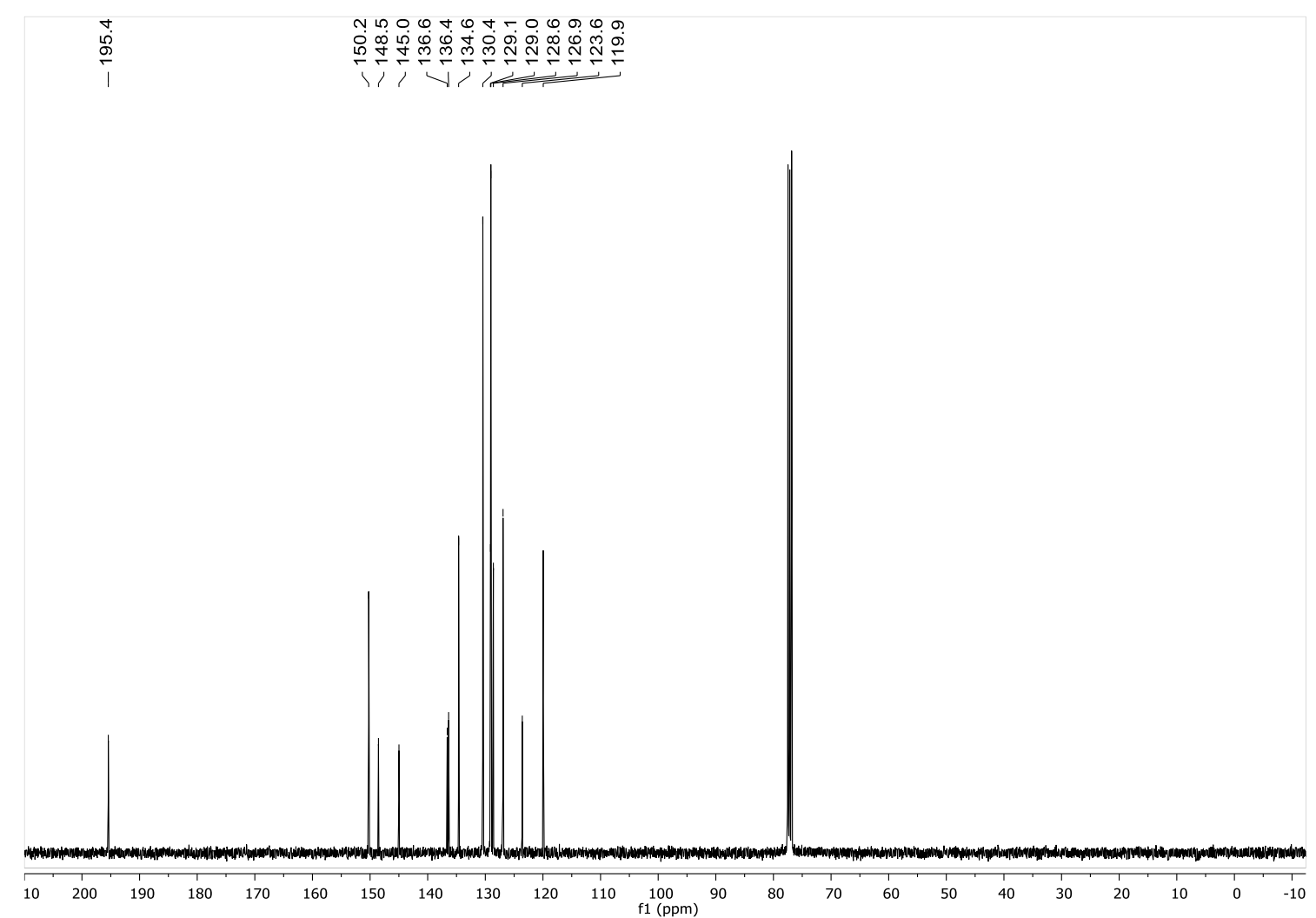


${ }^{1} \mathrm{H}$ NMR spectrum in $\mathrm{CDCl}_{3}$ of compound $\mathbf{1 0 b}$

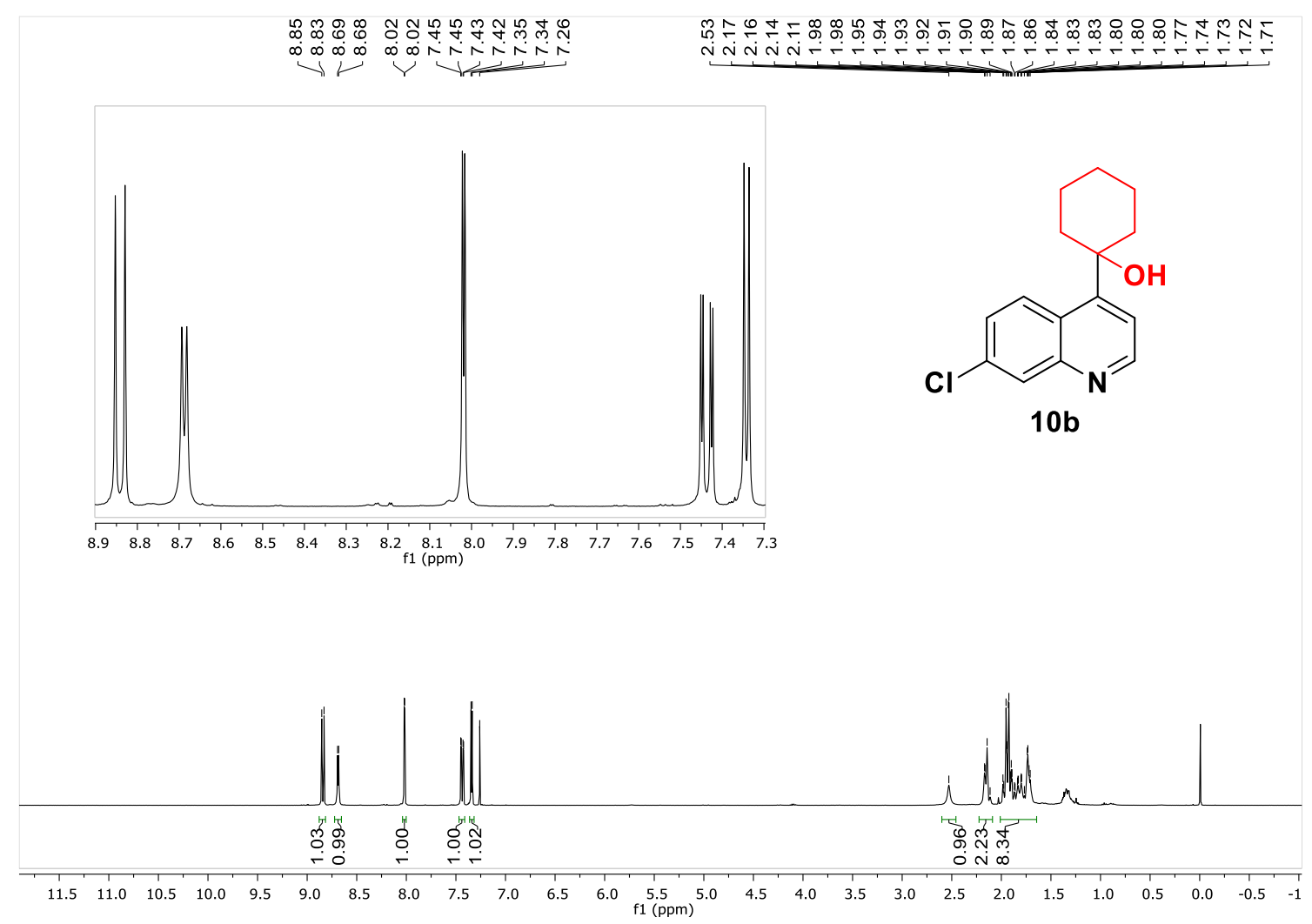

${ }^{13} \mathrm{C}\left\{{ }^{1} \mathrm{H}\right\}$ NMR spectrum in $\mathrm{CDCl}_{3}$ of compound $\mathbf{1 0 b}$

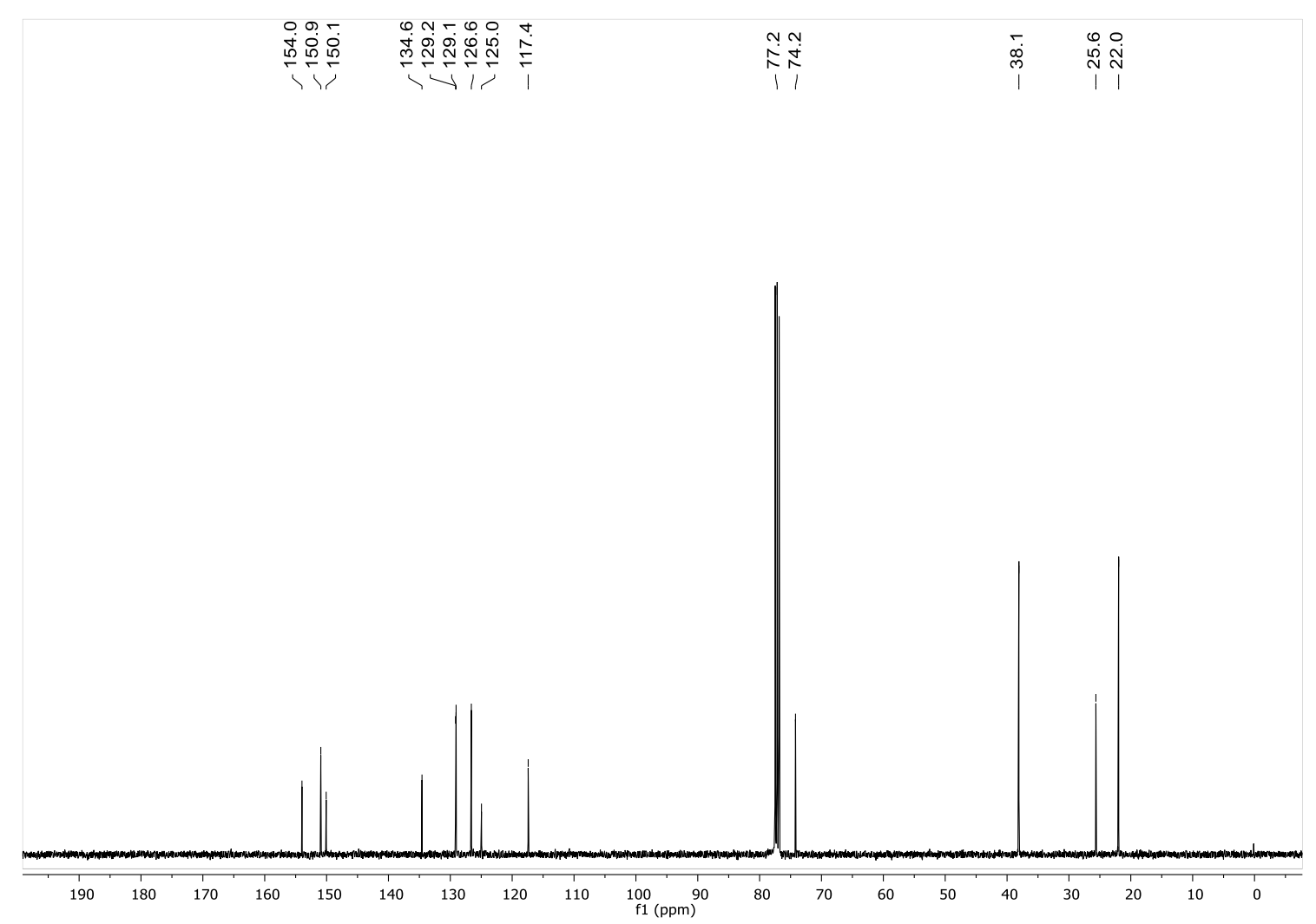


${ }^{1} \mathrm{H}$ NMR spectrum in $\mathrm{CDCl}_{3}$ of compound 10c

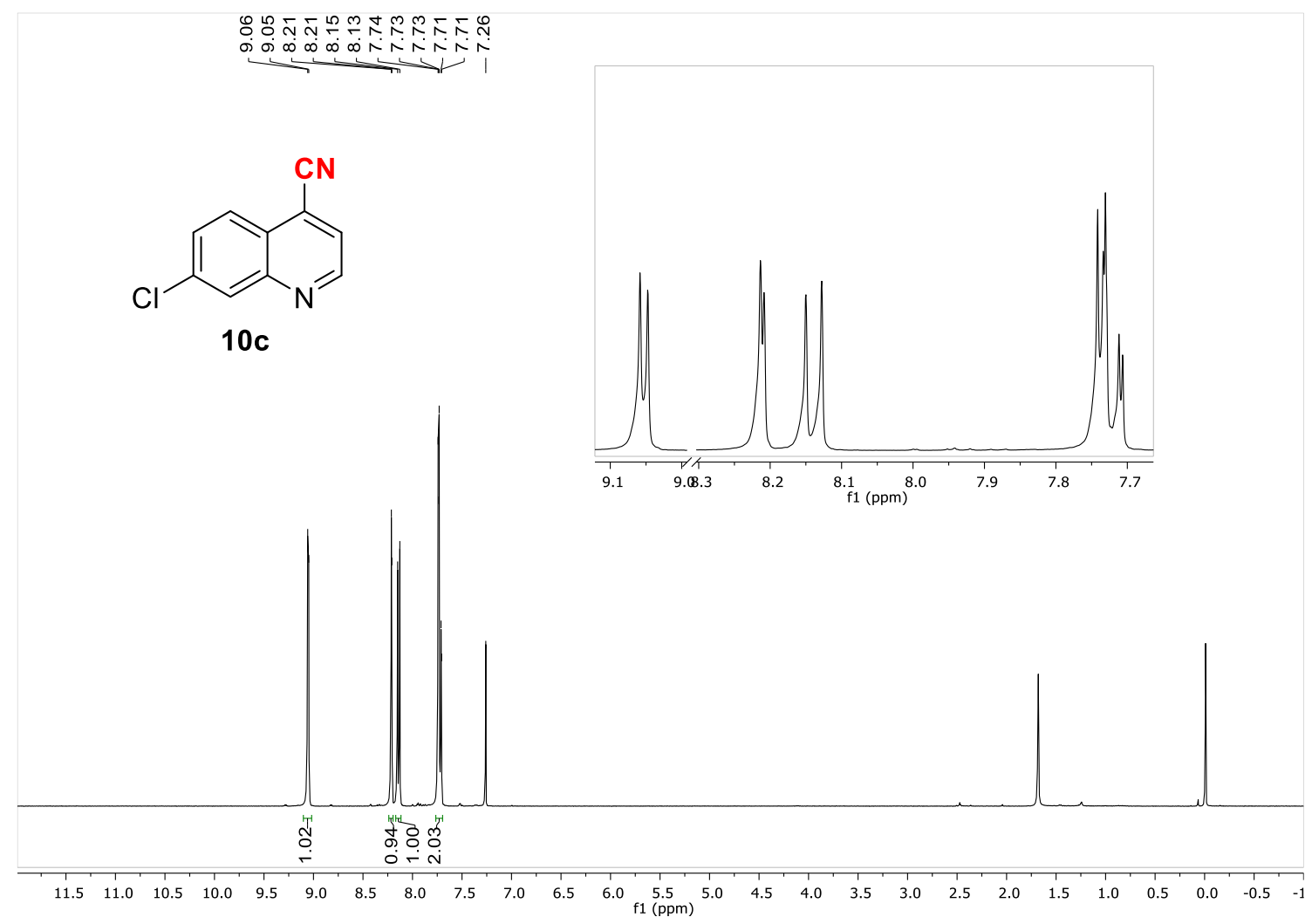

${ }^{13} \mathrm{C}\left\{{ }^{1} \mathrm{H}\right\}$ NMR spectrum in $\mathrm{CDCl}_{3}$ of compound 10c<smiles>N#Cc1ccnc2cc(Cl)ccc12</smiles>

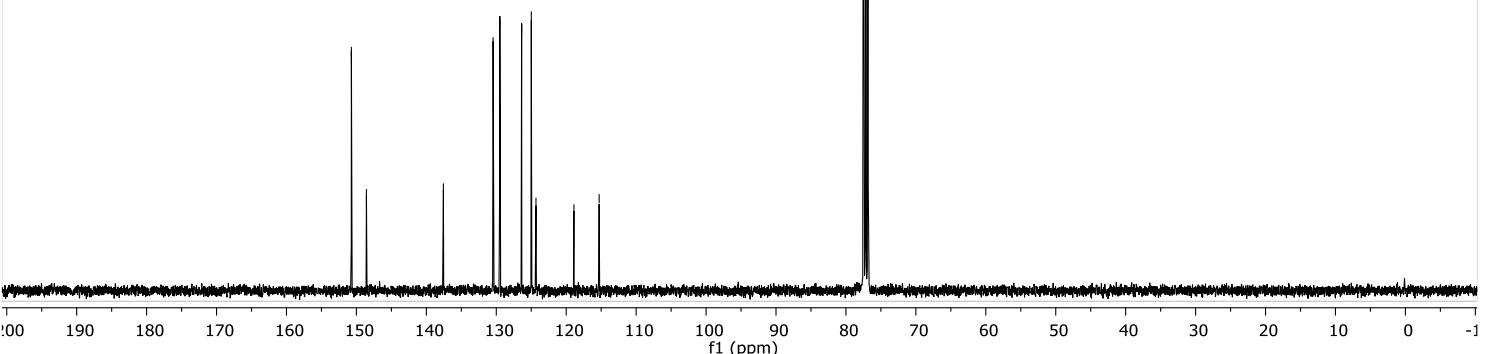


${ }^{1} \mathrm{H}$ NMR spectrum in $\mathrm{CDCl}_{3}$ of compound 10d

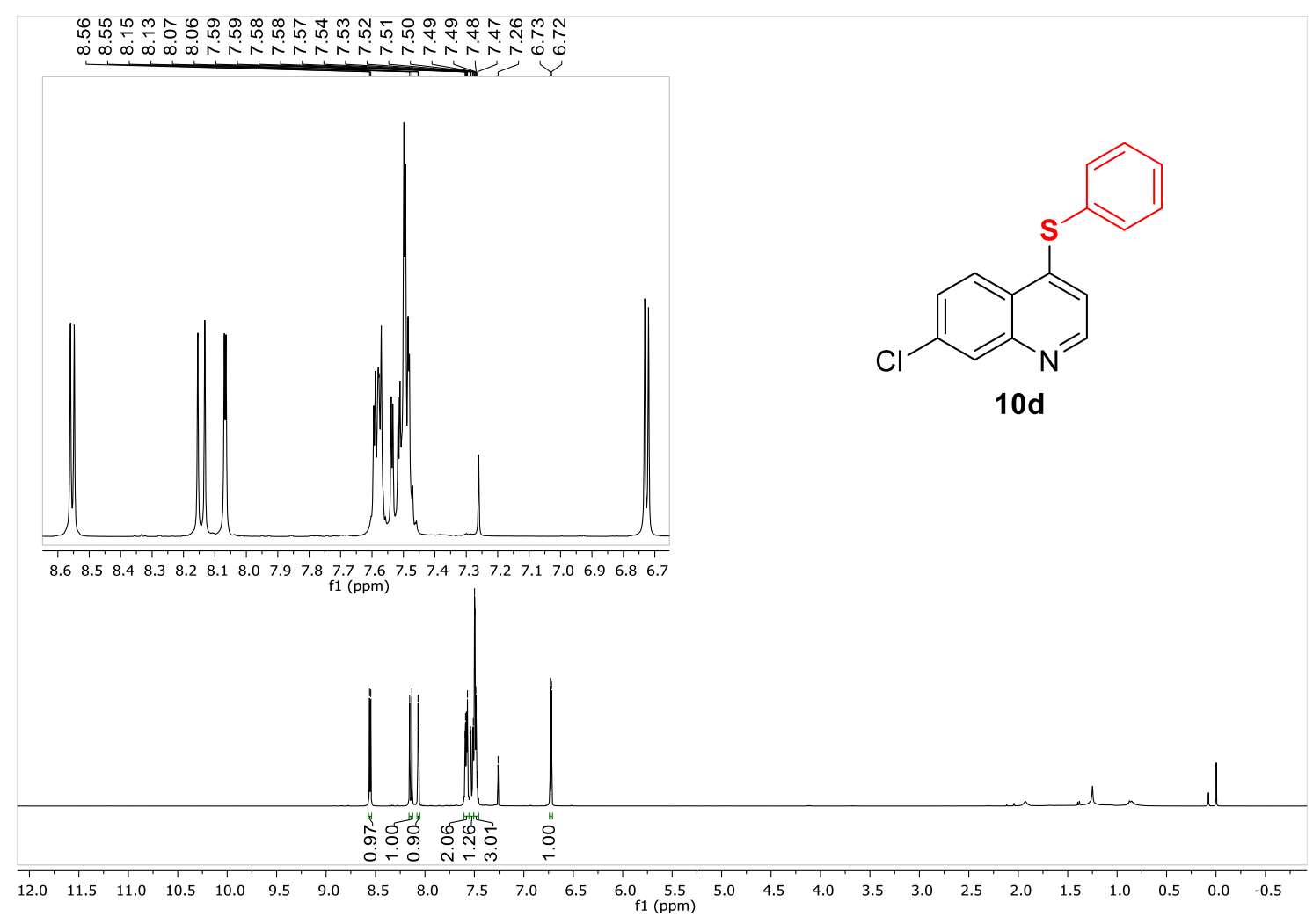

${ }^{13} \mathrm{C}\left\{{ }^{1} \mathrm{H}\right\}$ NMR spectrum in $\mathrm{CDCl}_{3}$ of compound $\mathbf{1 0 d}$

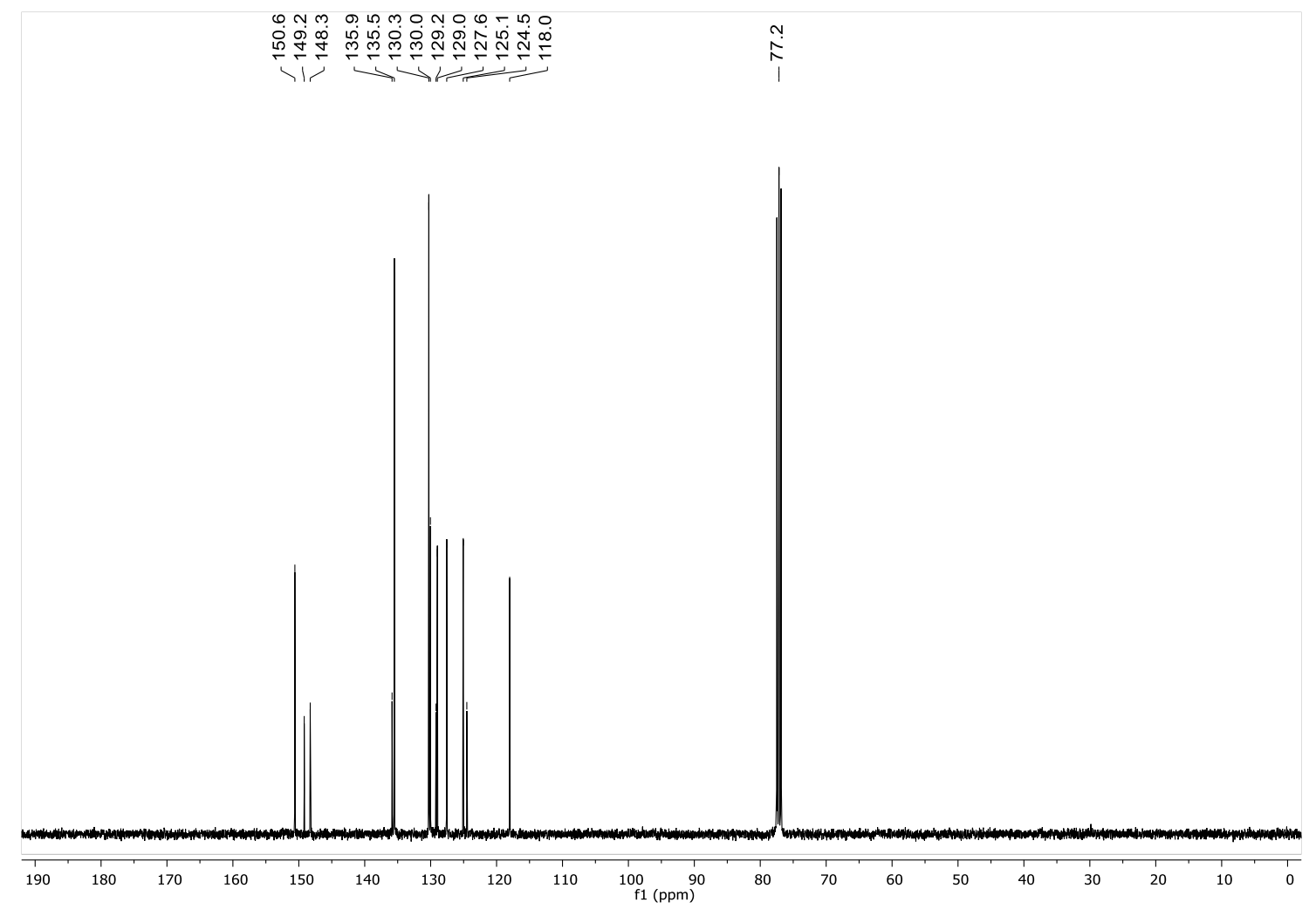


${ }^{1} \mathrm{H}$ NMR spectrum in $\mathrm{CDCl}_{3}$ of compound 10e

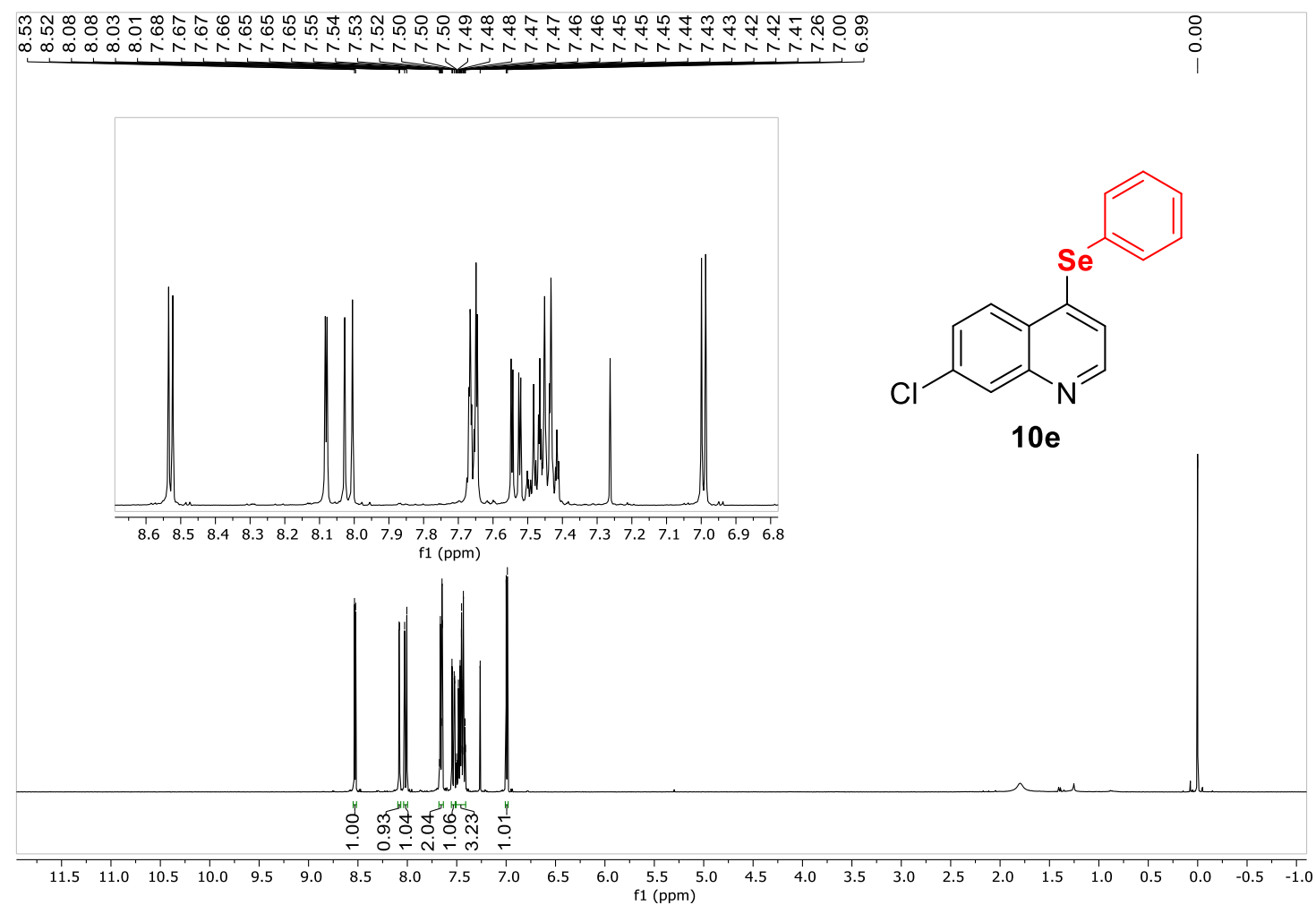

${ }^{13} \mathrm{C}\left\{{ }^{1} \mathrm{H}\right\}$ NMR spectrum in $\mathrm{CDCl}_{3}$ of compound 10e

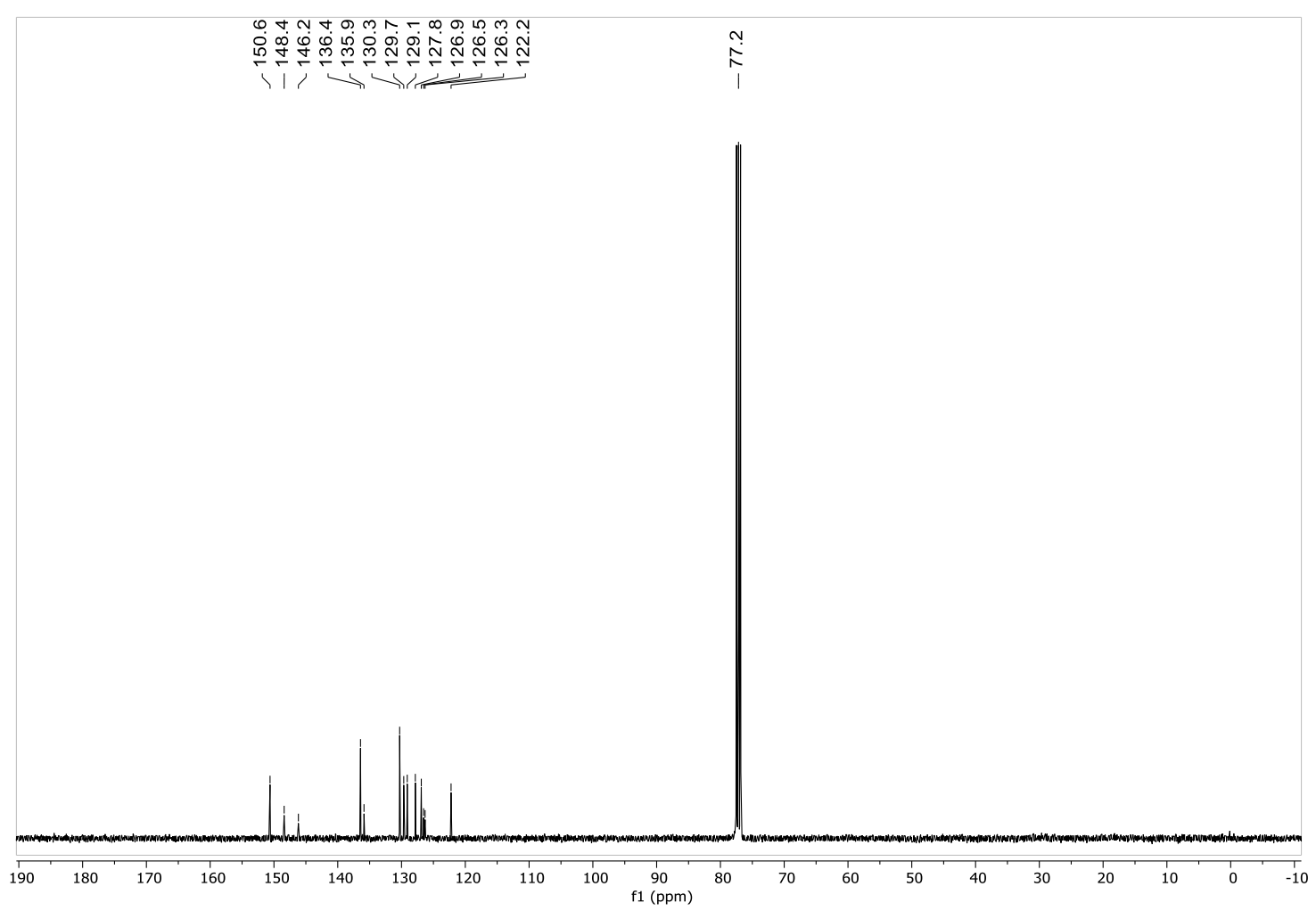


${ }^{1} \mathrm{H}$ NMR spectrum in $\mathrm{CDCl}_{3}$ of compound $\mathbf{1 0 f}$

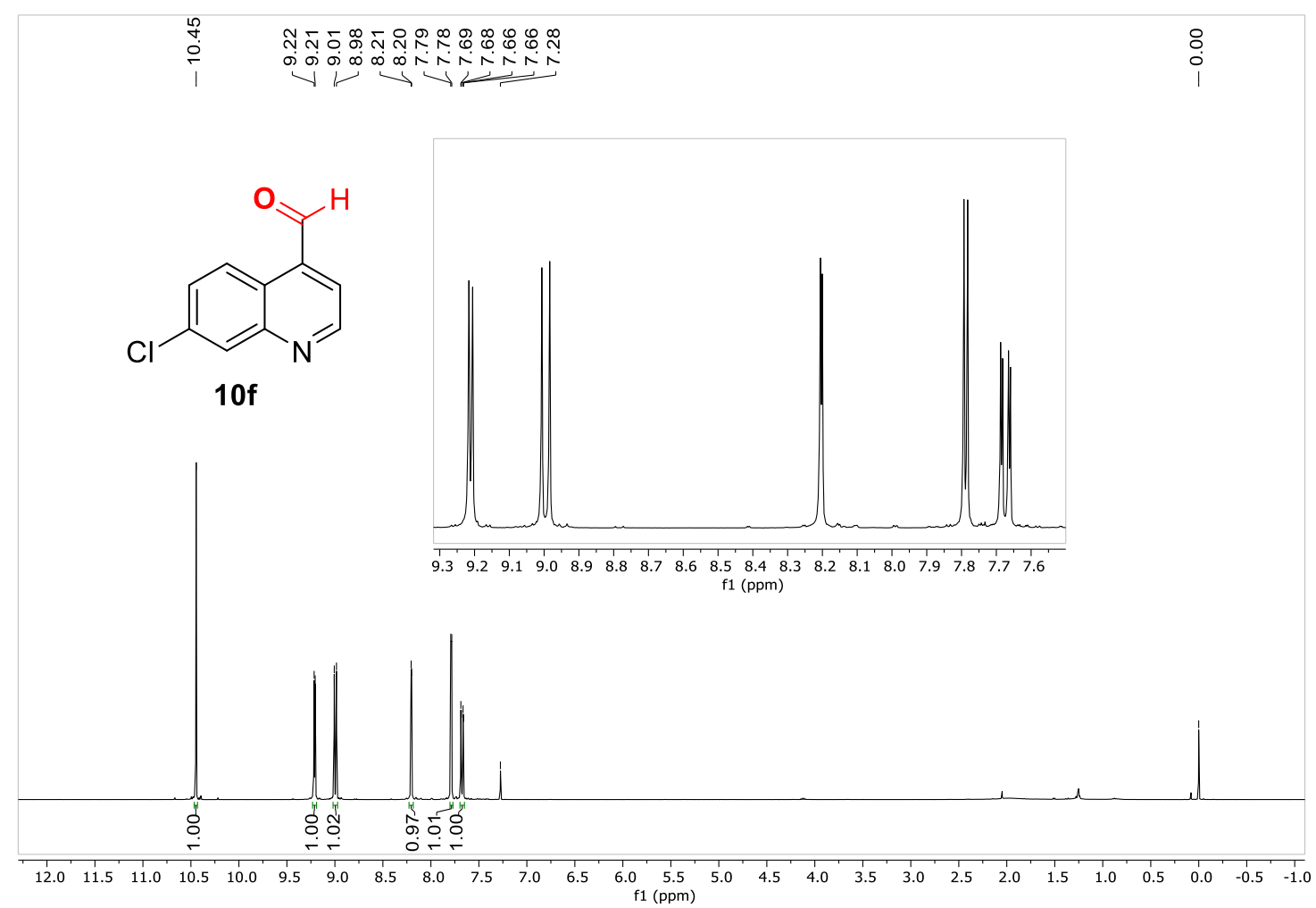

${ }^{13} \mathrm{C}\left\{{ }^{1} \mathrm{H}\right\}$ NMR spectrum in $\mathrm{CDCl}_{3}$ of compound $\mathbf{1 0 f}$

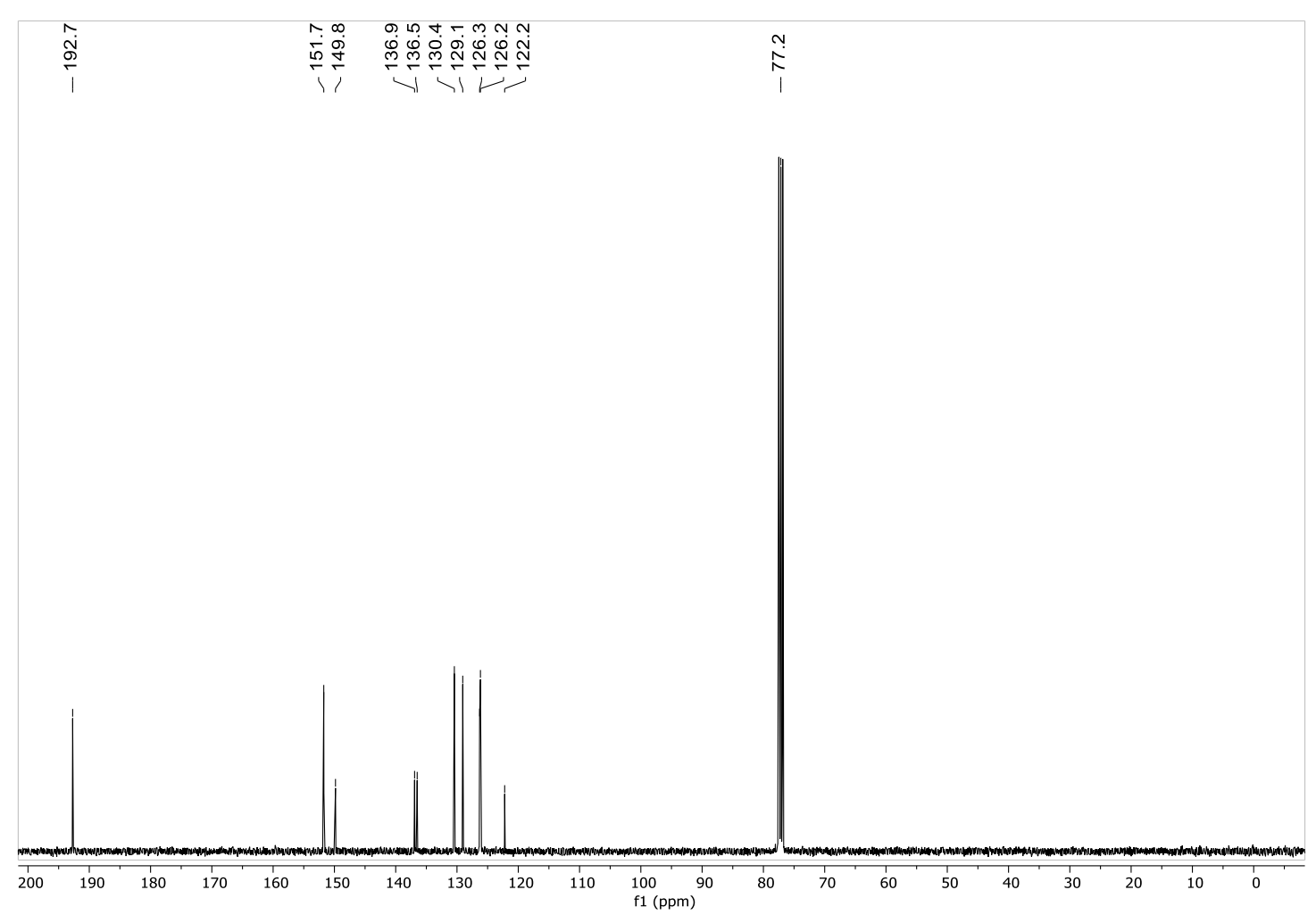


${ }^{1} \mathrm{H}$ NMR spectrum in $\mathrm{CDCl}_{3}$ of compound $\mathbf{1 0 g}$

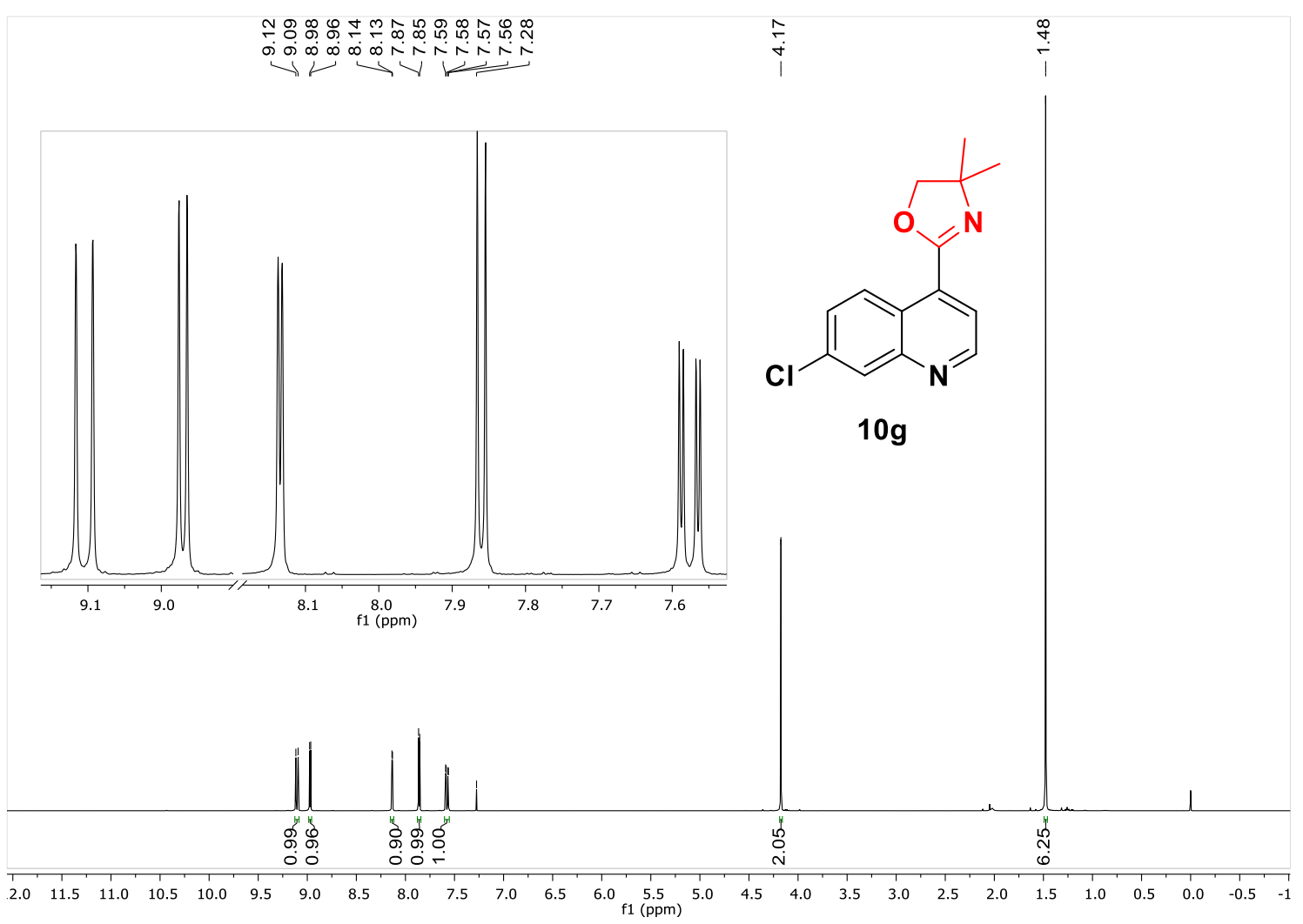

${ }^{13} \mathrm{C}\left\{{ }^{1} \mathrm{H}\right\}$ NMR spectrum in $\mathrm{CDCl}_{3}$ of compound $\mathbf{1 0 g}$

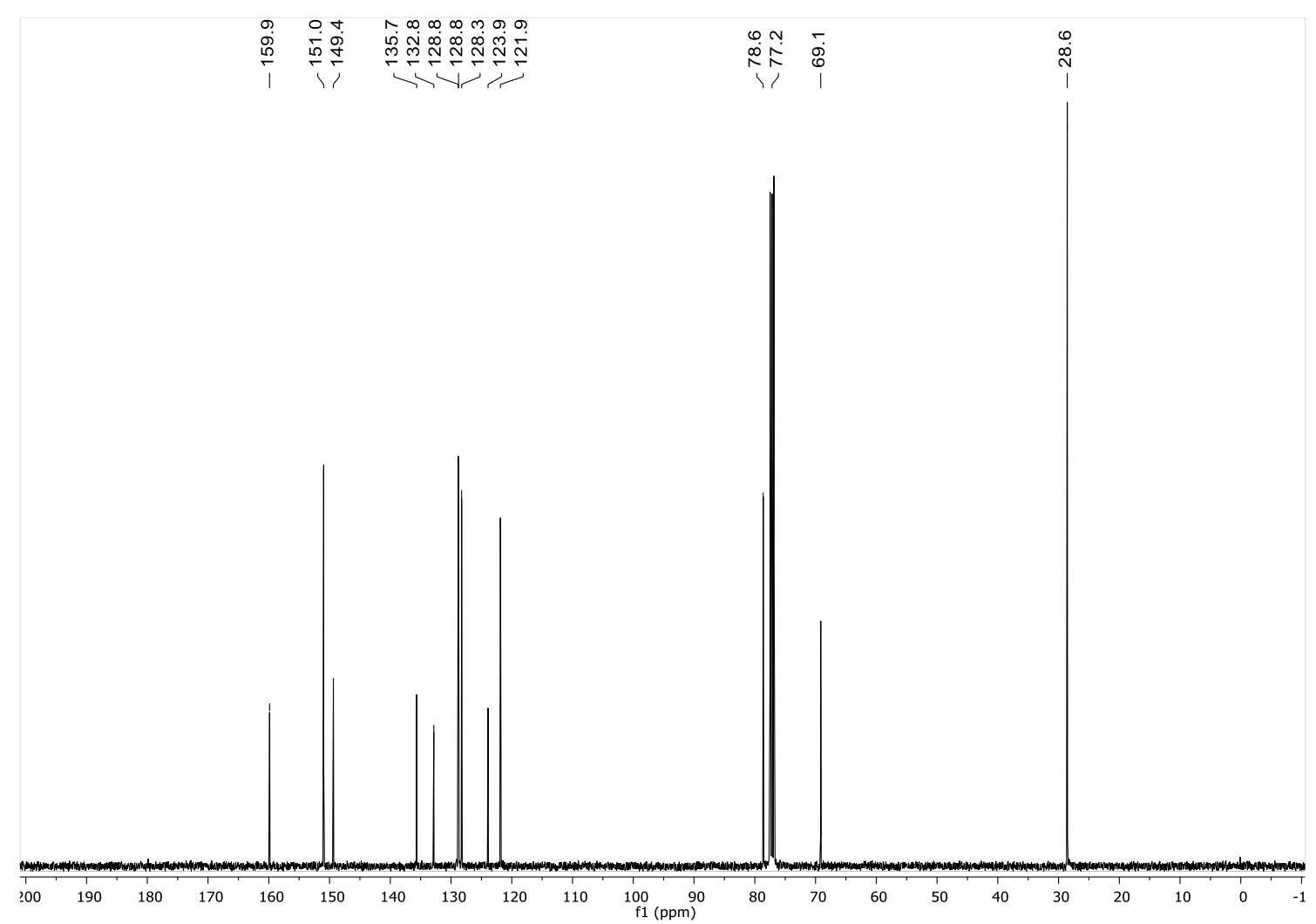


${ }^{1} \mathrm{H}$ NMR spectrum in $\mathrm{CDCl}_{3}$ of compound $\mathbf{1 0 h}$

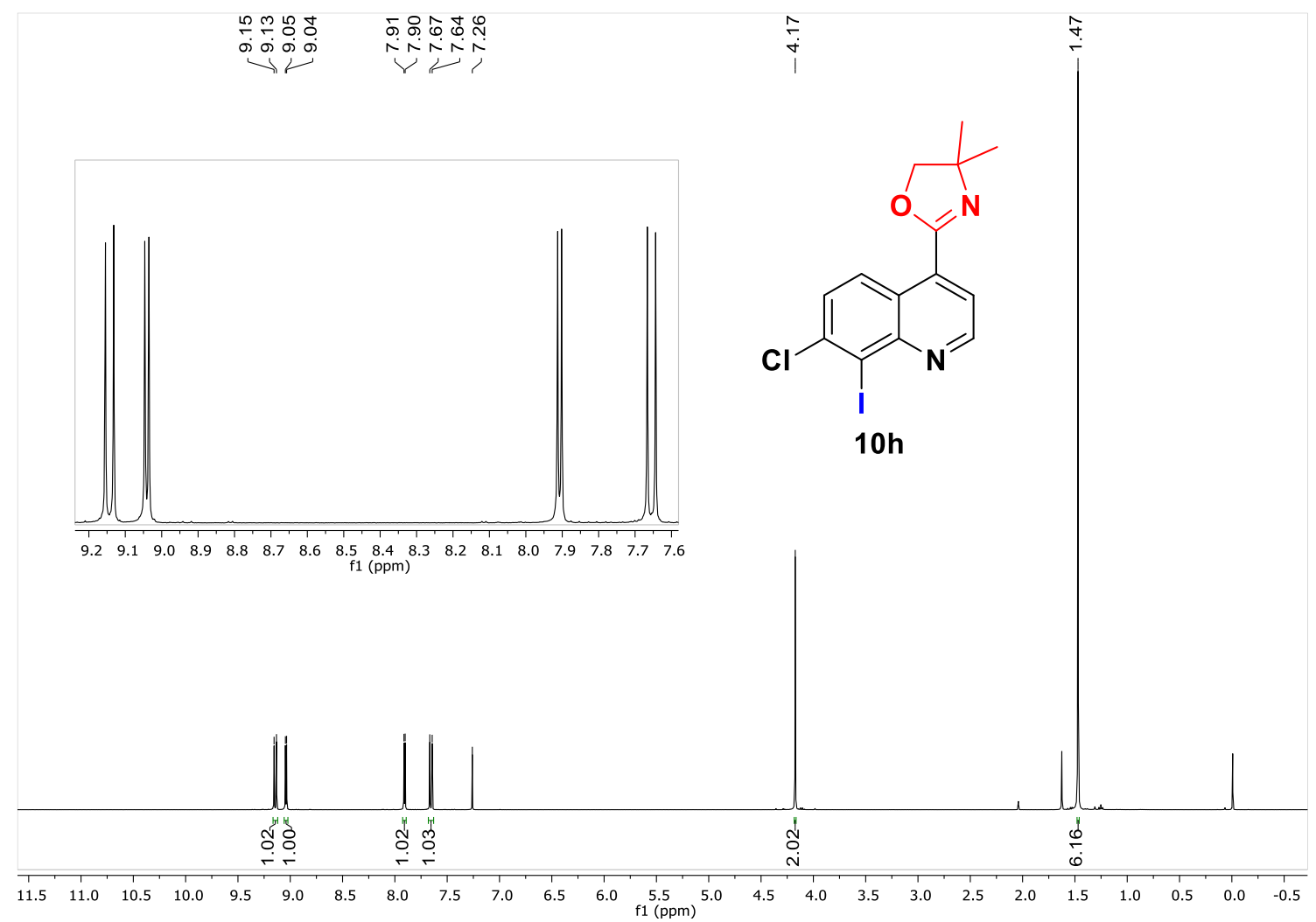

${ }^{13} \mathrm{C}\left\{{ }^{1} \mathrm{H}\right\}$ NMR spectrum in $\mathrm{CDCl}_{3}$ of compound $\mathbf{1 0 h}$

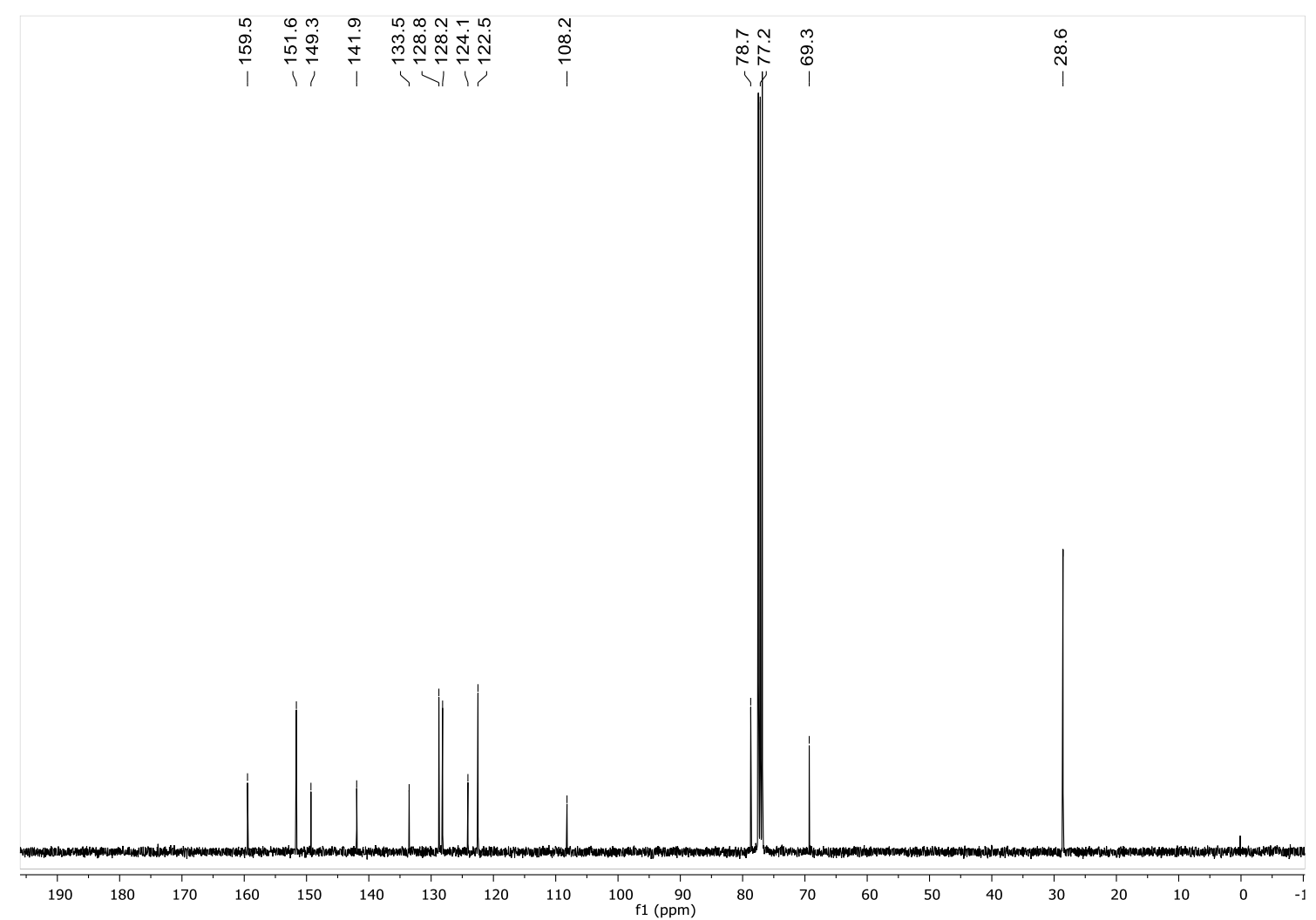


${ }^{1} \mathrm{H}$ NMR spectrum in $\mathrm{CDCl}_{3}$ of compound 12a

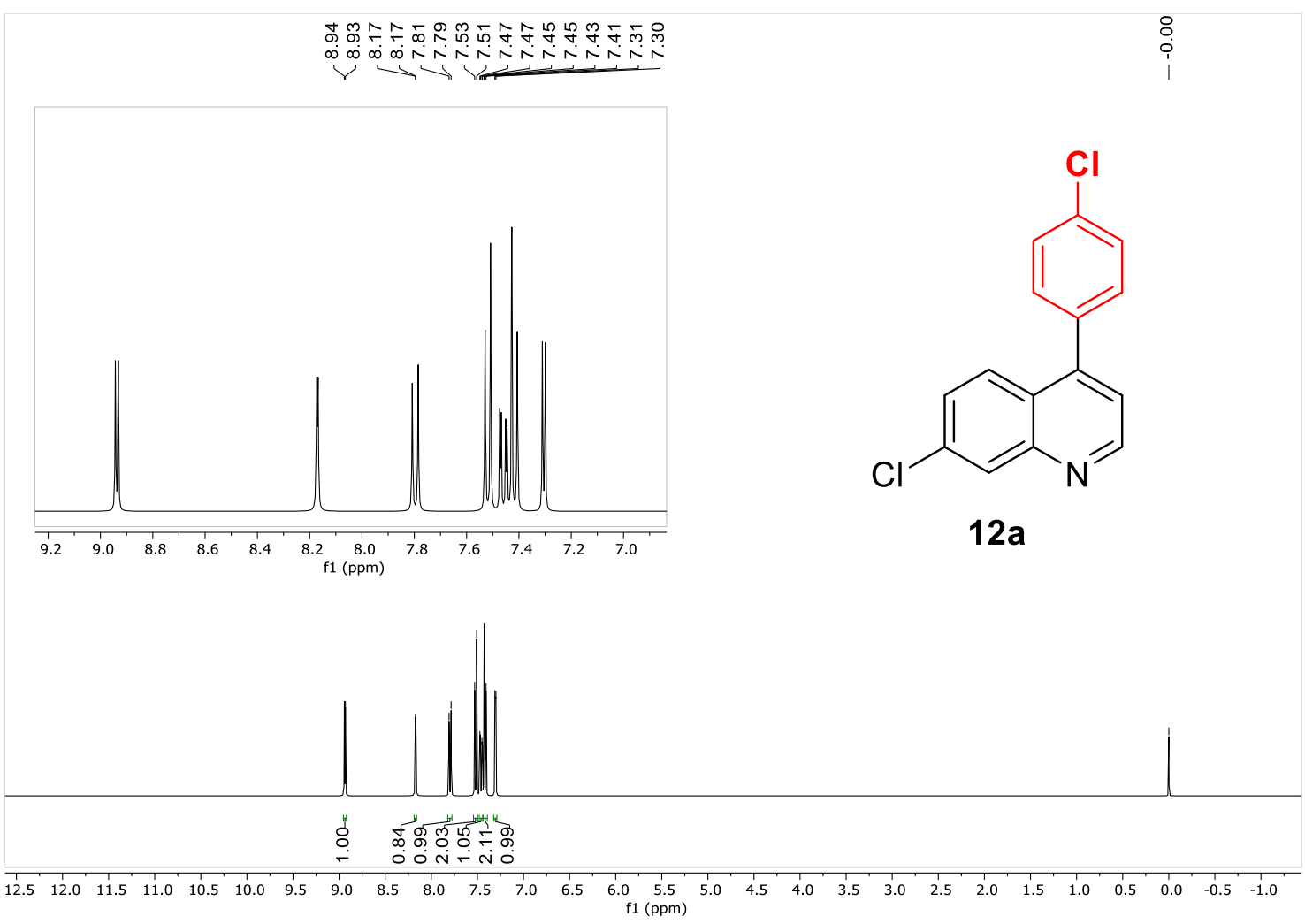

${ }^{13} \mathrm{C}\left\{{ }^{1} \mathrm{H}\right\} \mathrm{NMR}$ spectrum in $\mathrm{CDCl}_{3}$ of compound 12a

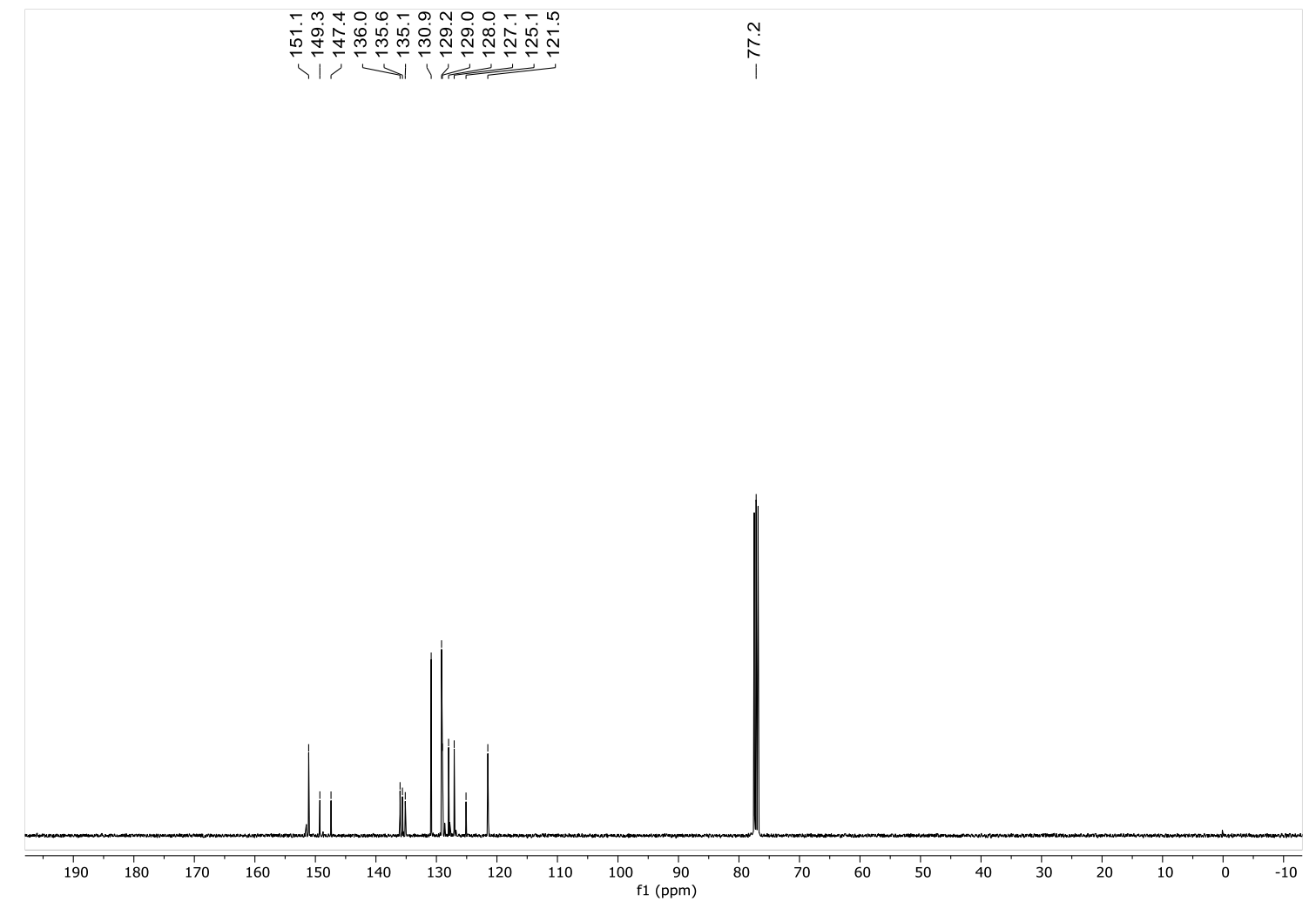


${ }^{1} \mathrm{H}$ NMR spectrum in $\mathrm{CDCl}_{3}$ of compound $\mathbf{1 2 b}$

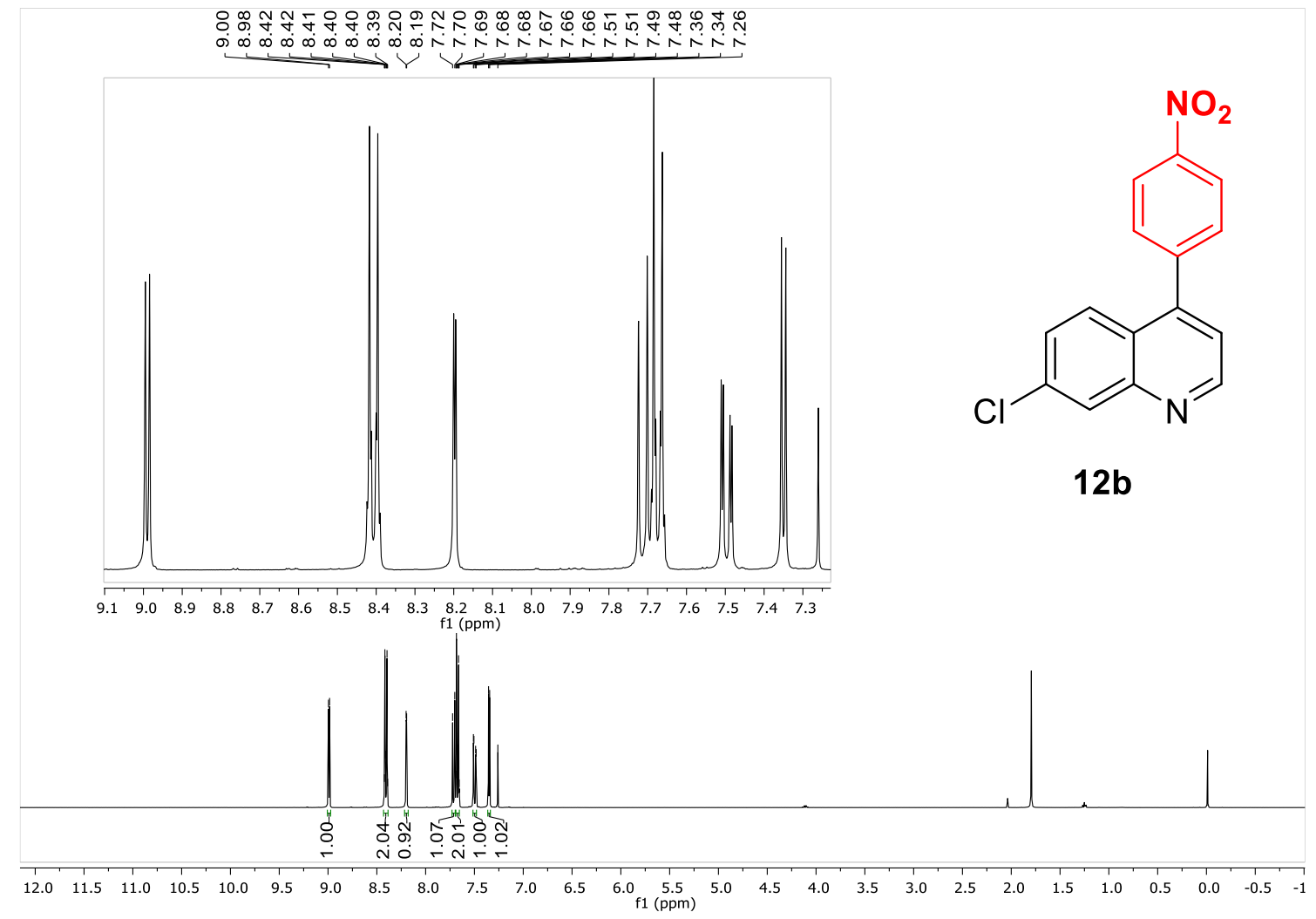

${ }^{13} \mathrm{C}\left\{{ }^{1} \mathrm{H}\right\}$ NMR spectrum in $\mathrm{CDCl}_{3}$ of compound $\mathbf{1 2 b}$

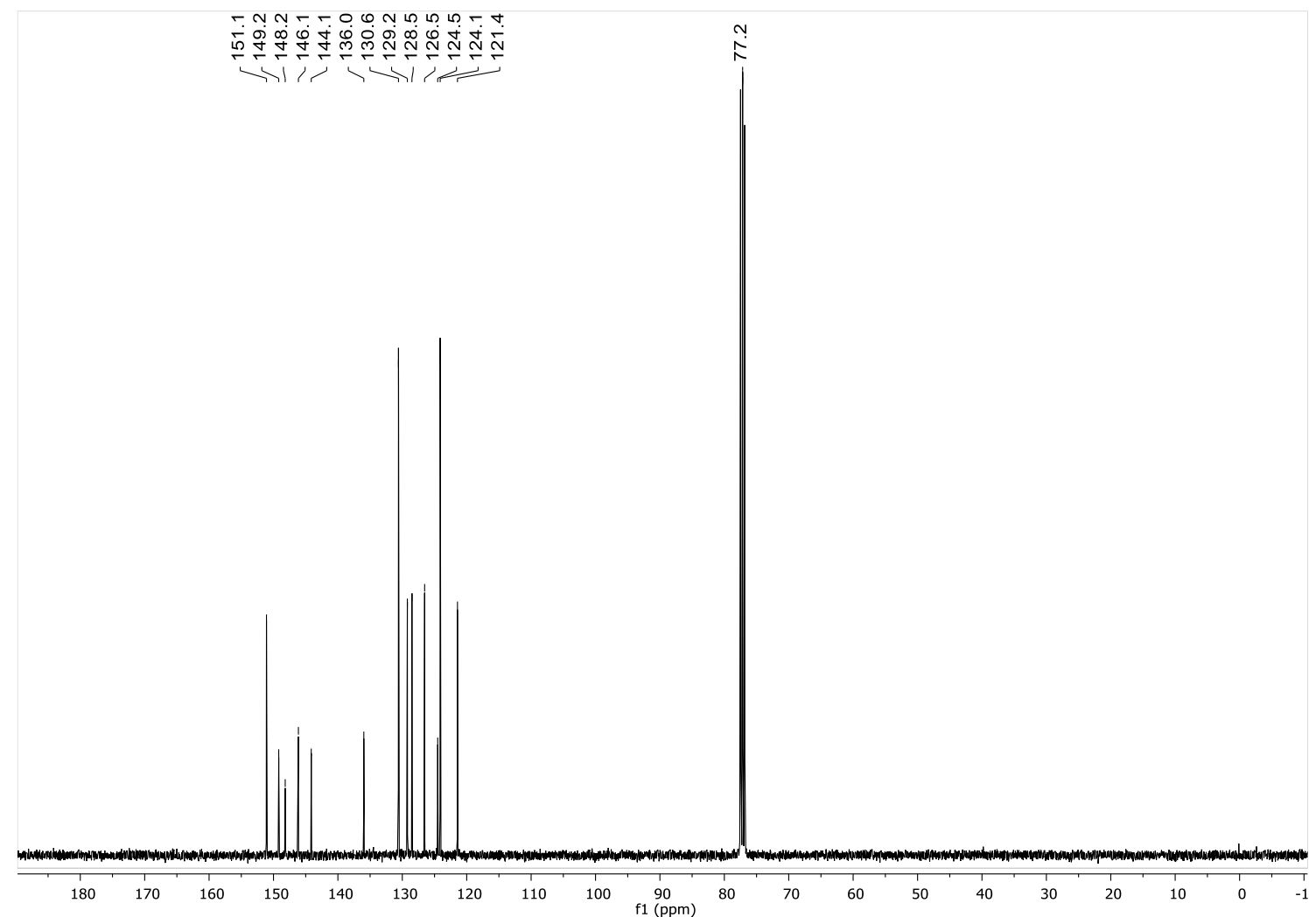


${ }^{1} \mathrm{H}$ NMR spectrum in $\mathrm{CDCl}_{3}$ of compound $\mathbf{1 2 c}$

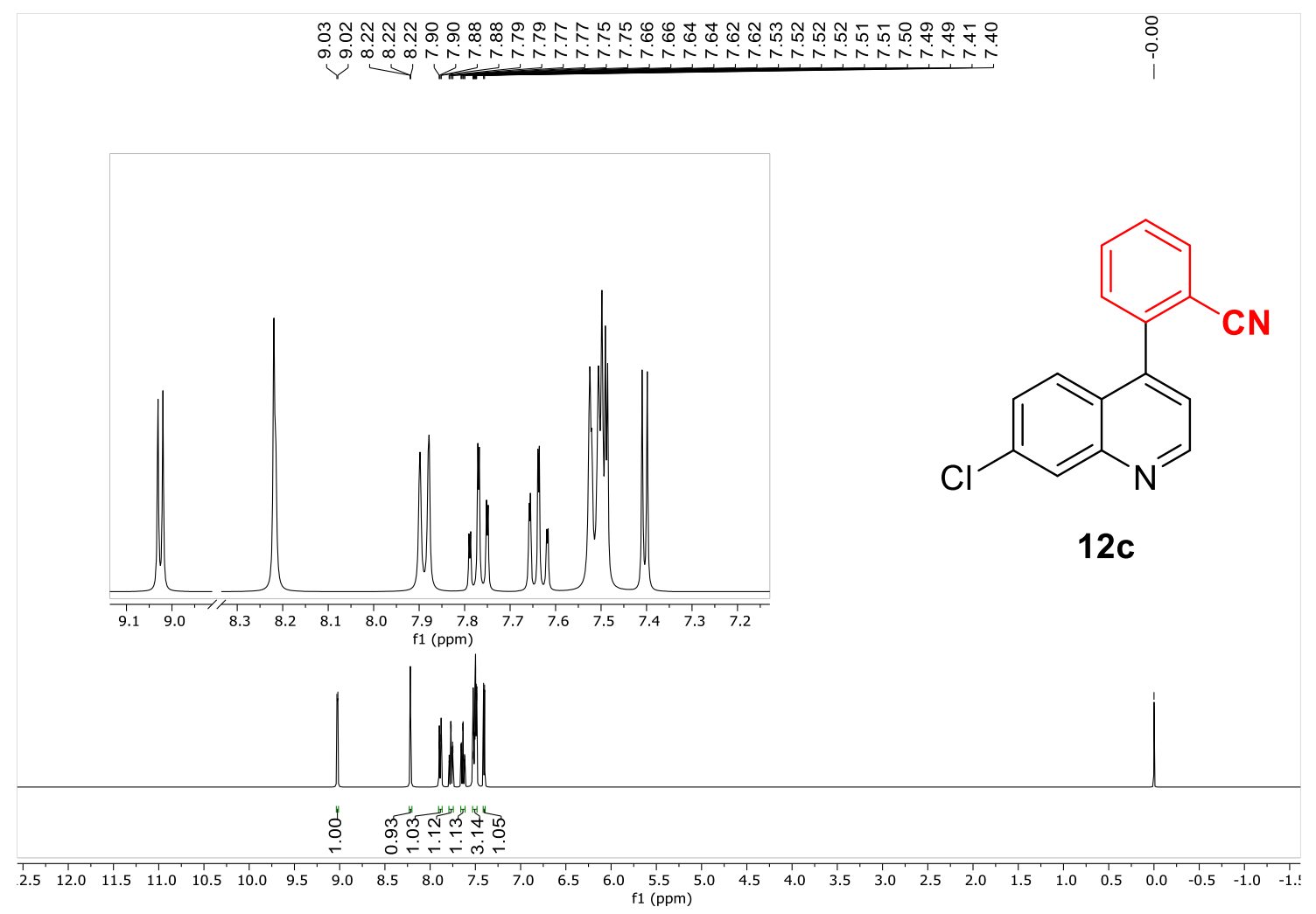

${ }^{13} \mathrm{C}\left\{{ }^{1} \mathrm{H}\right\}$ NMR spectrum in $\mathrm{CDCl}_{3}$ of compound $\mathbf{1 2 c}$

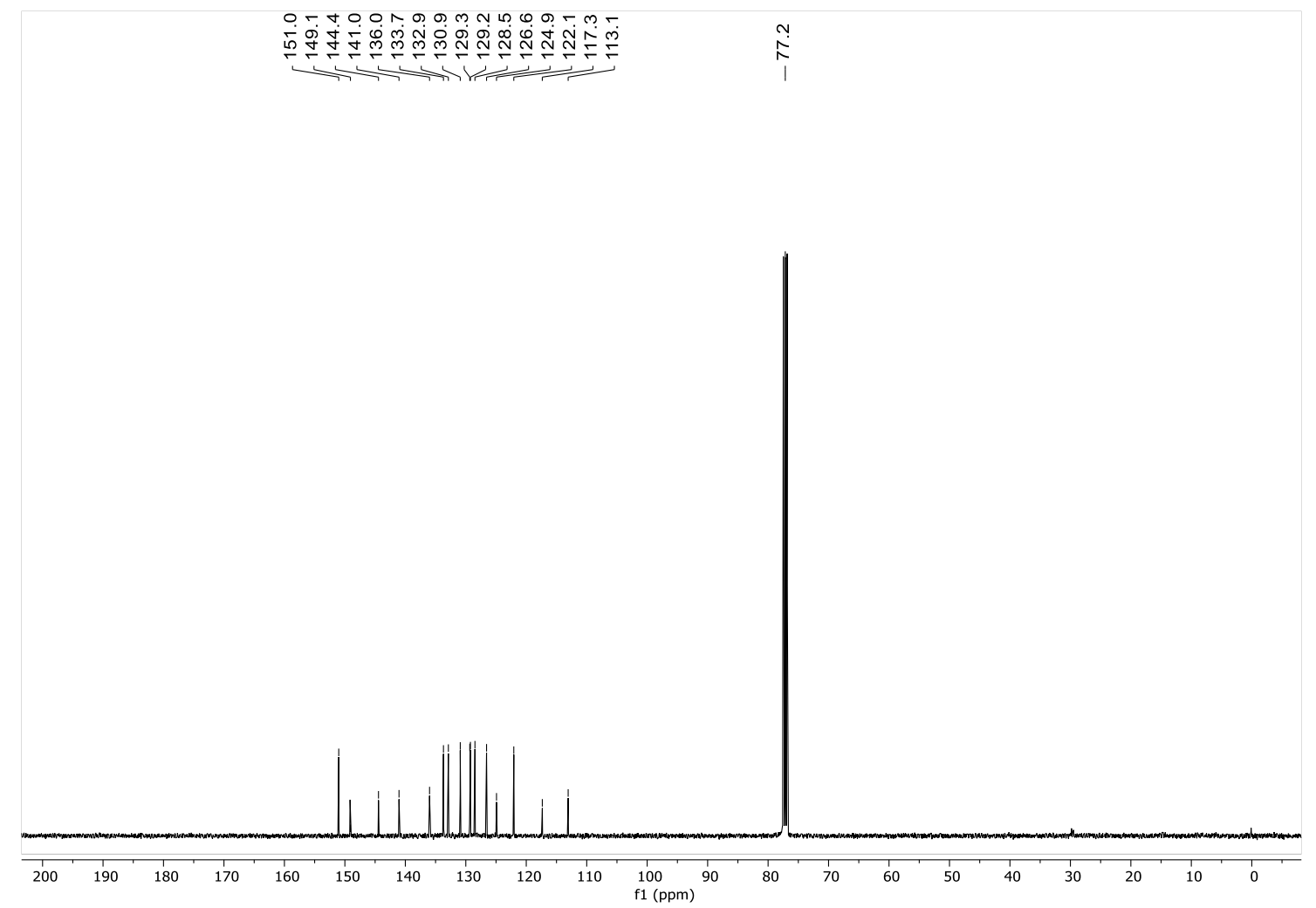


${ }^{1} \mathrm{H}$ NMR spectrum in $\mathrm{CDCl}_{3}$ of compound $\mathbf{1 2 d}$

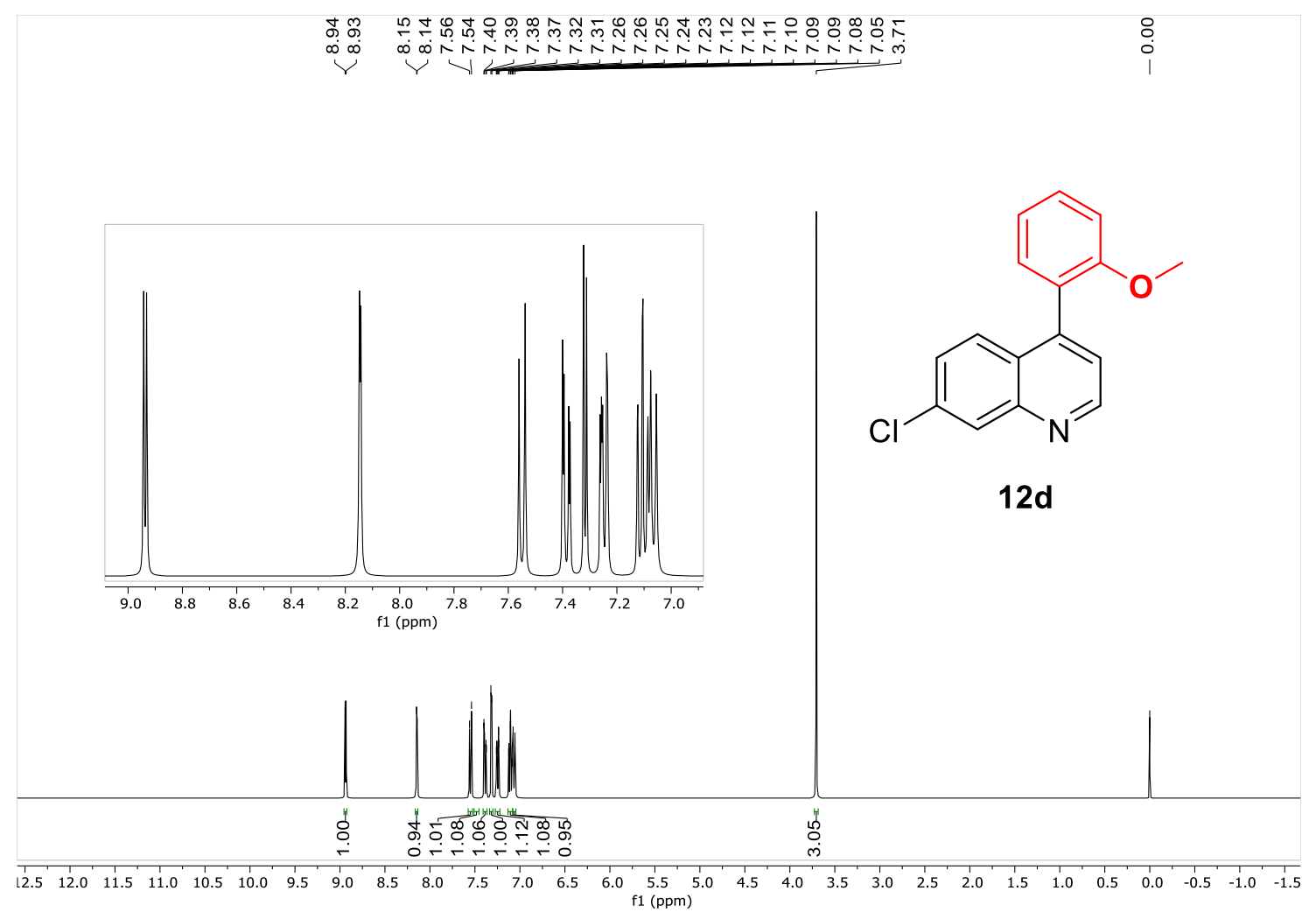

${ }^{13} \mathrm{C}\left\{{ }^{1} \mathrm{H}\right\}$ NMR spectrum in $\mathrm{CDCl}_{3}$ of compound $\mathbf{1 2 d}$

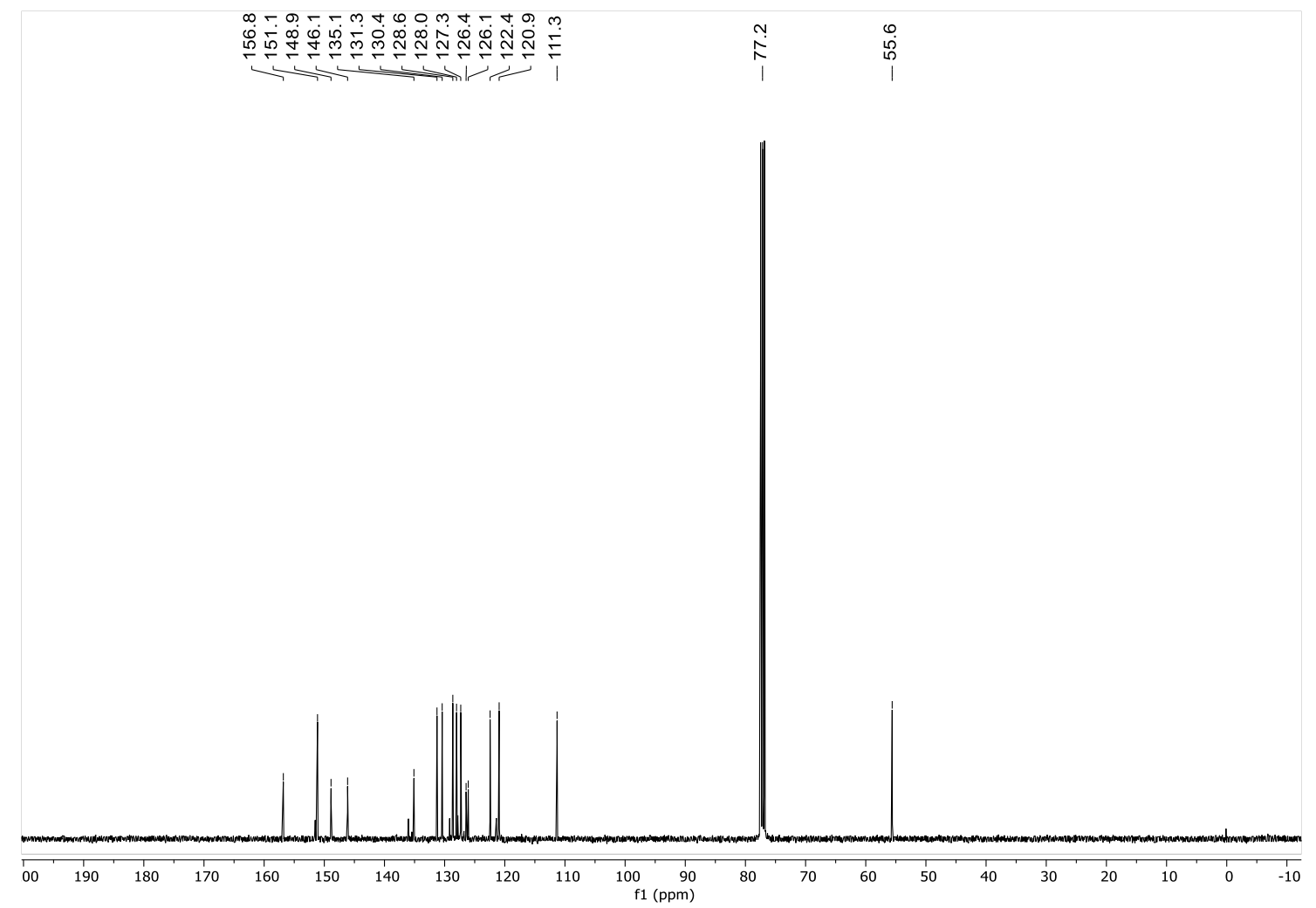


${ }^{1} \mathrm{H}$ NMR spectrum in DMSO- $\mathrm{d}_{6}$ of compound $\mathbf{1 6}$

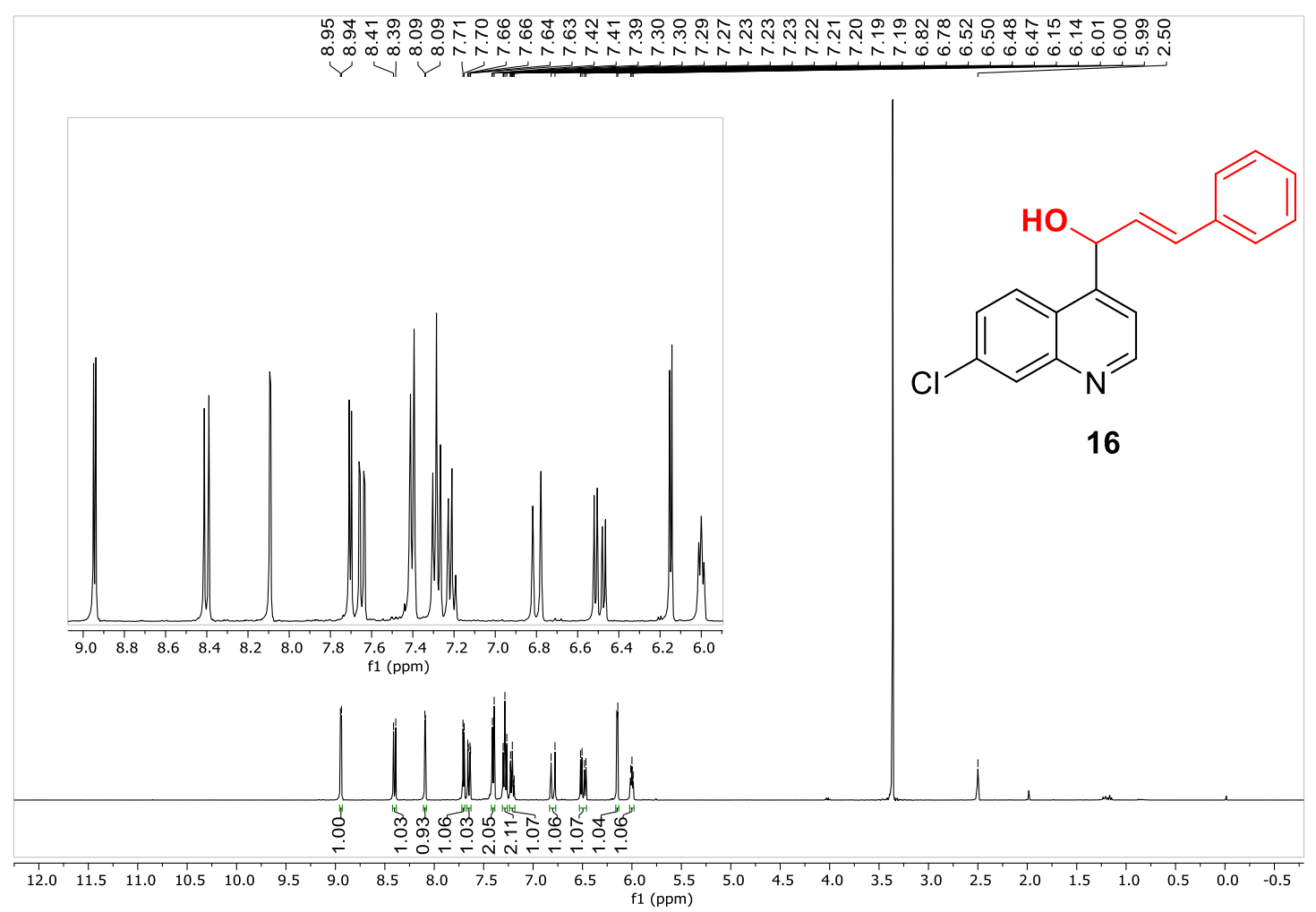

${ }^{13} \mathrm{C}\left\{{ }^{1} \mathrm{H}\right\}$ NMR spectrum in DMSO-d 6 of compound $\mathbf{1 6}$

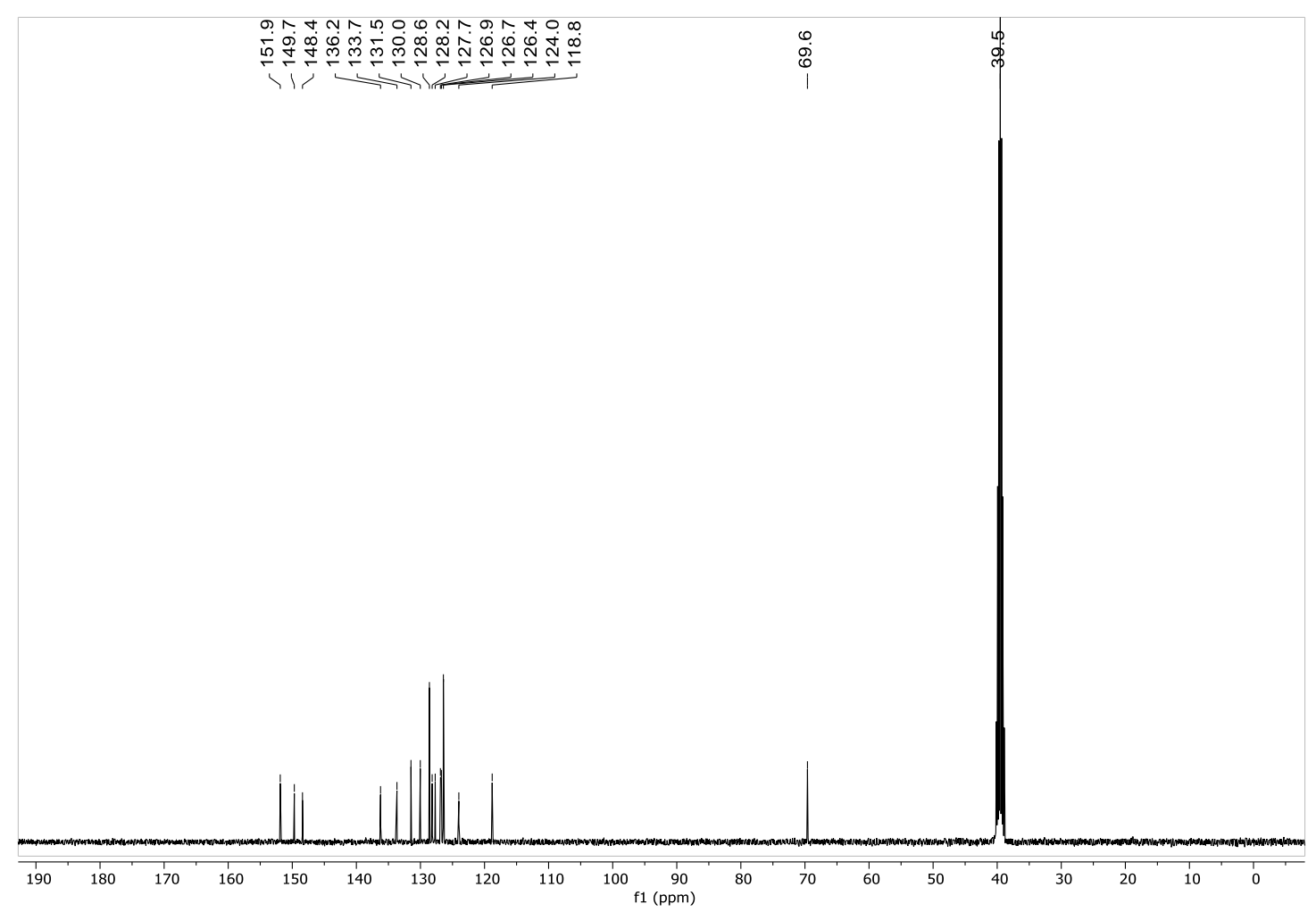


${ }^{1} \mathrm{H}$ NMR spectrum in $\mathrm{CDCl}_{3}$ of compound 17

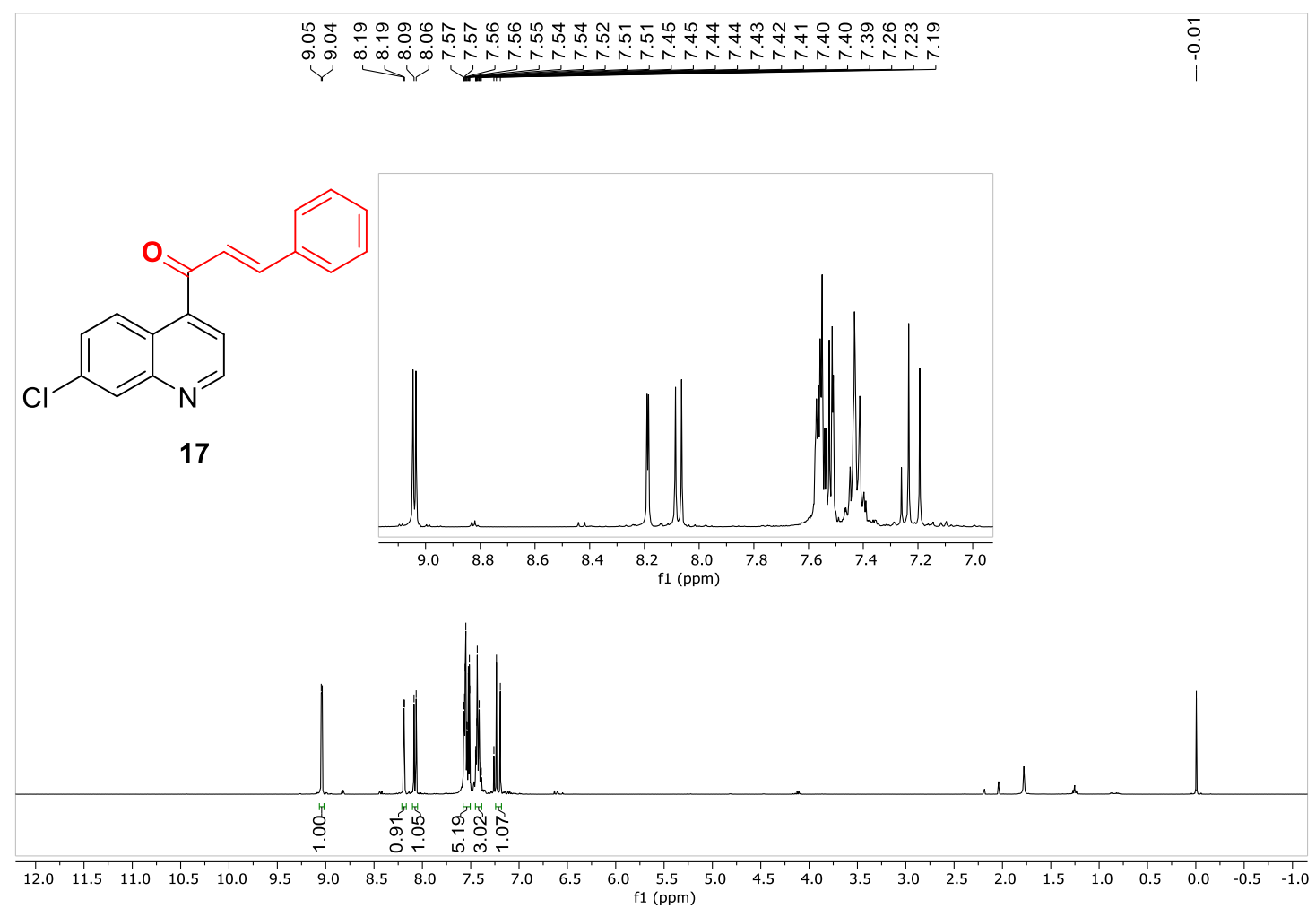

${ }^{13} \mathrm{C}\left\{{ }^{1} \mathrm{H}\right\}$ NMR spectrum in $\mathrm{CDCl}_{3}$ of compound $\mathbf{1 7}$

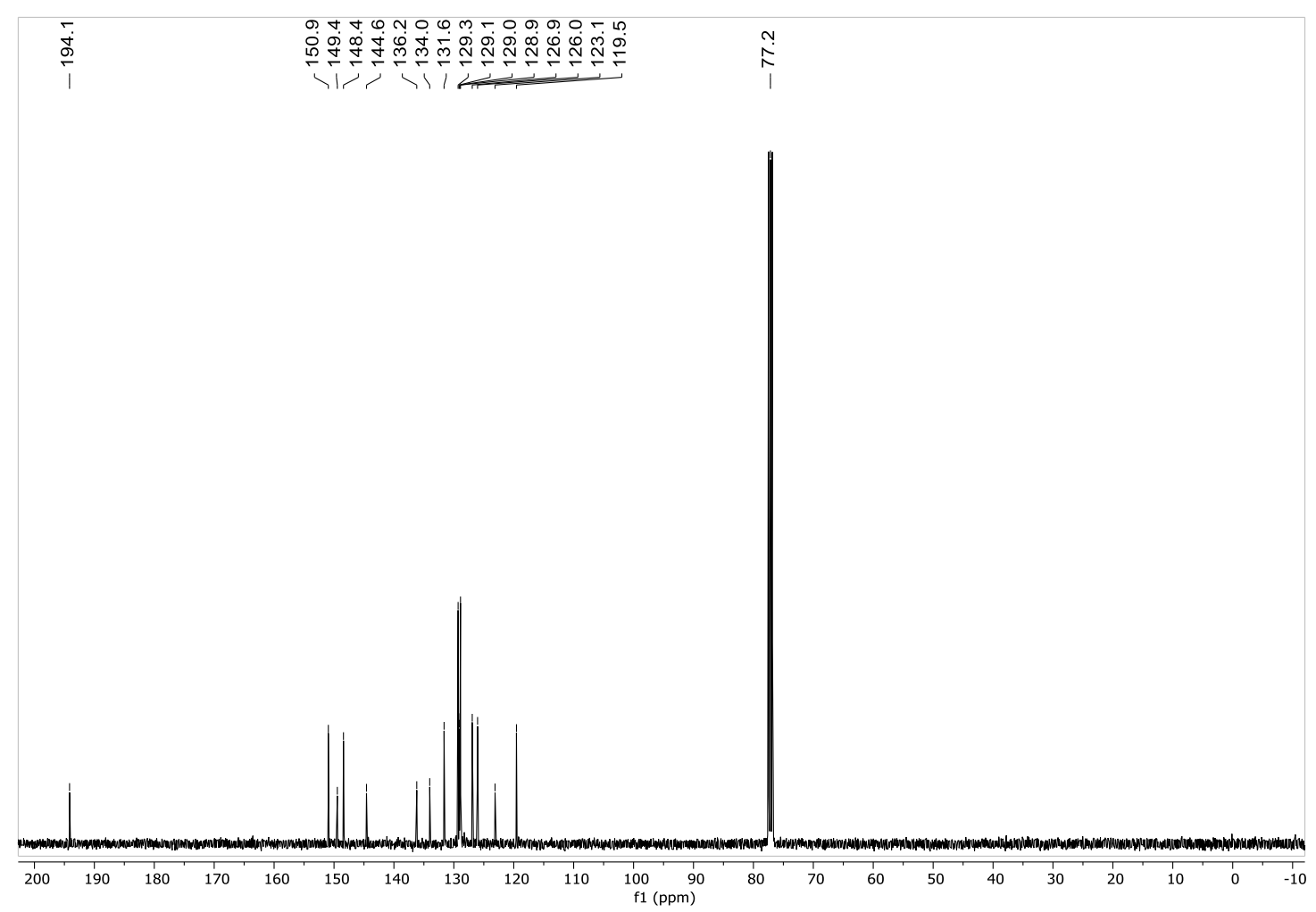


${ }^{1} \mathrm{H}$ NMR spectrum in DMSO- $\mathrm{d}_{6}$ of compound 19

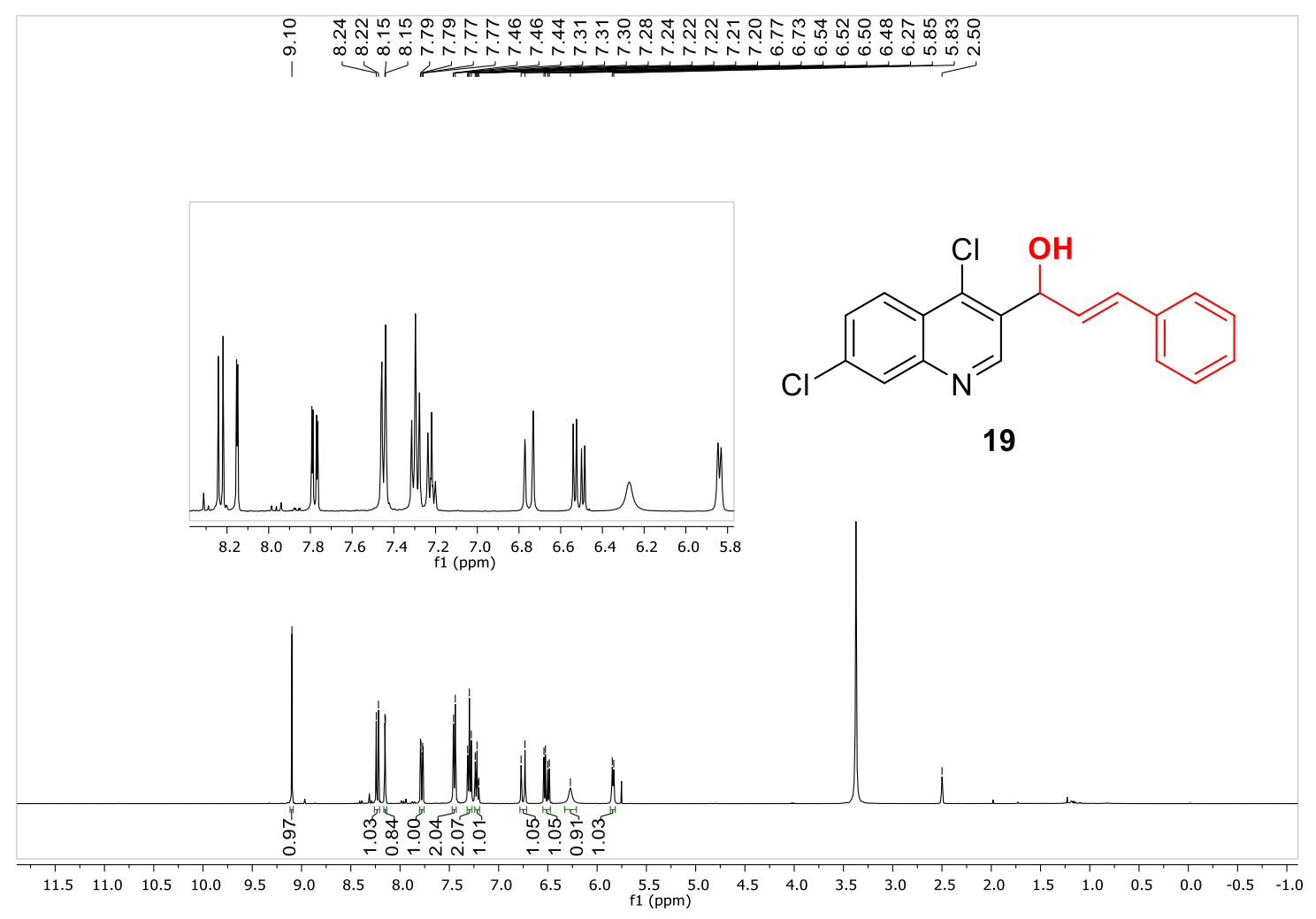

${ }^{13} \mathrm{C}\left\{{ }^{1} \mathrm{H}\right\}$ NMR spectrum in DMSO- $\mathrm{d}_{6}$ of compound 19

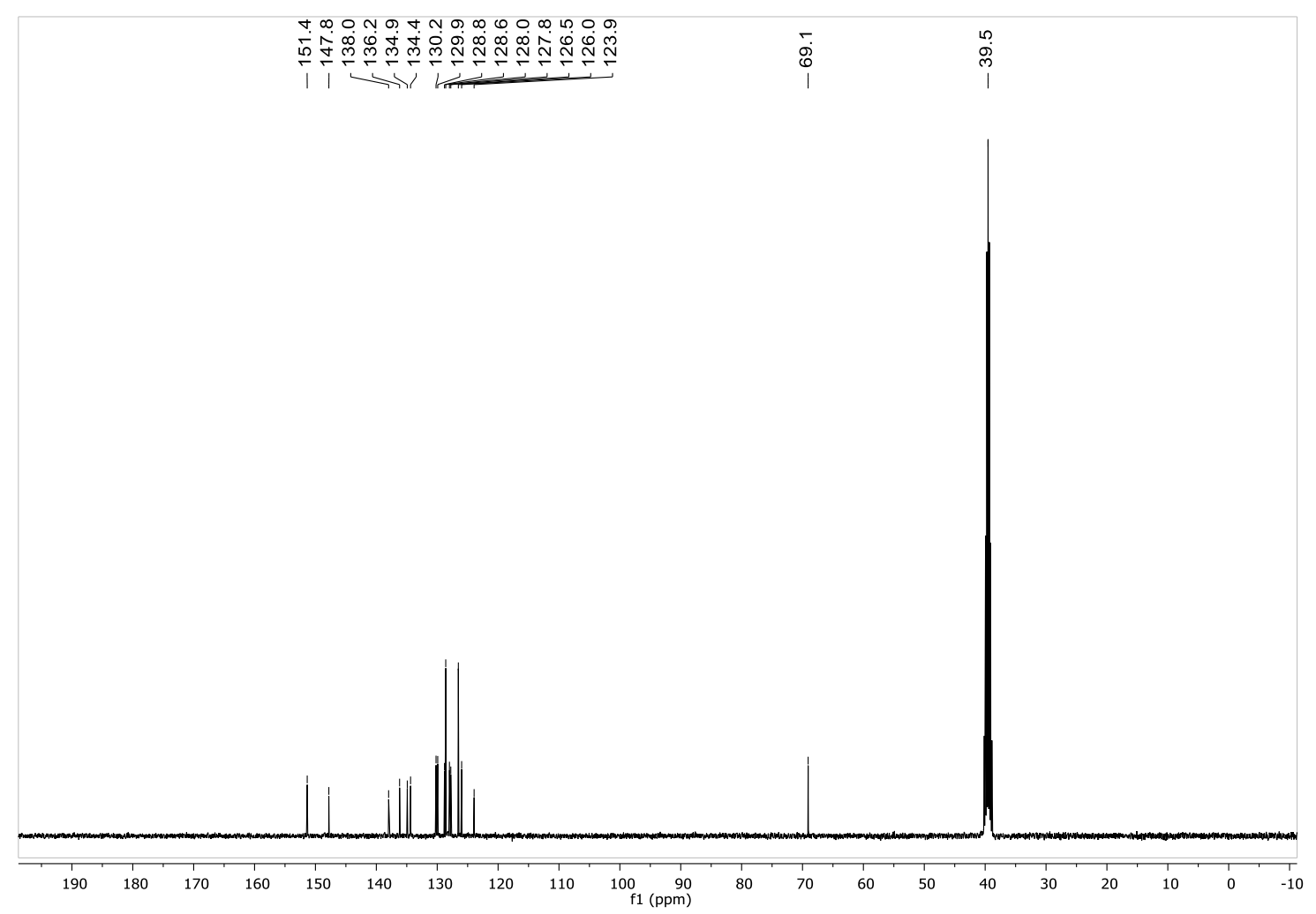


${ }^{1} \mathrm{H}$ NMR spectrum in $\mathrm{CDCl}_{3}$ of compound 20

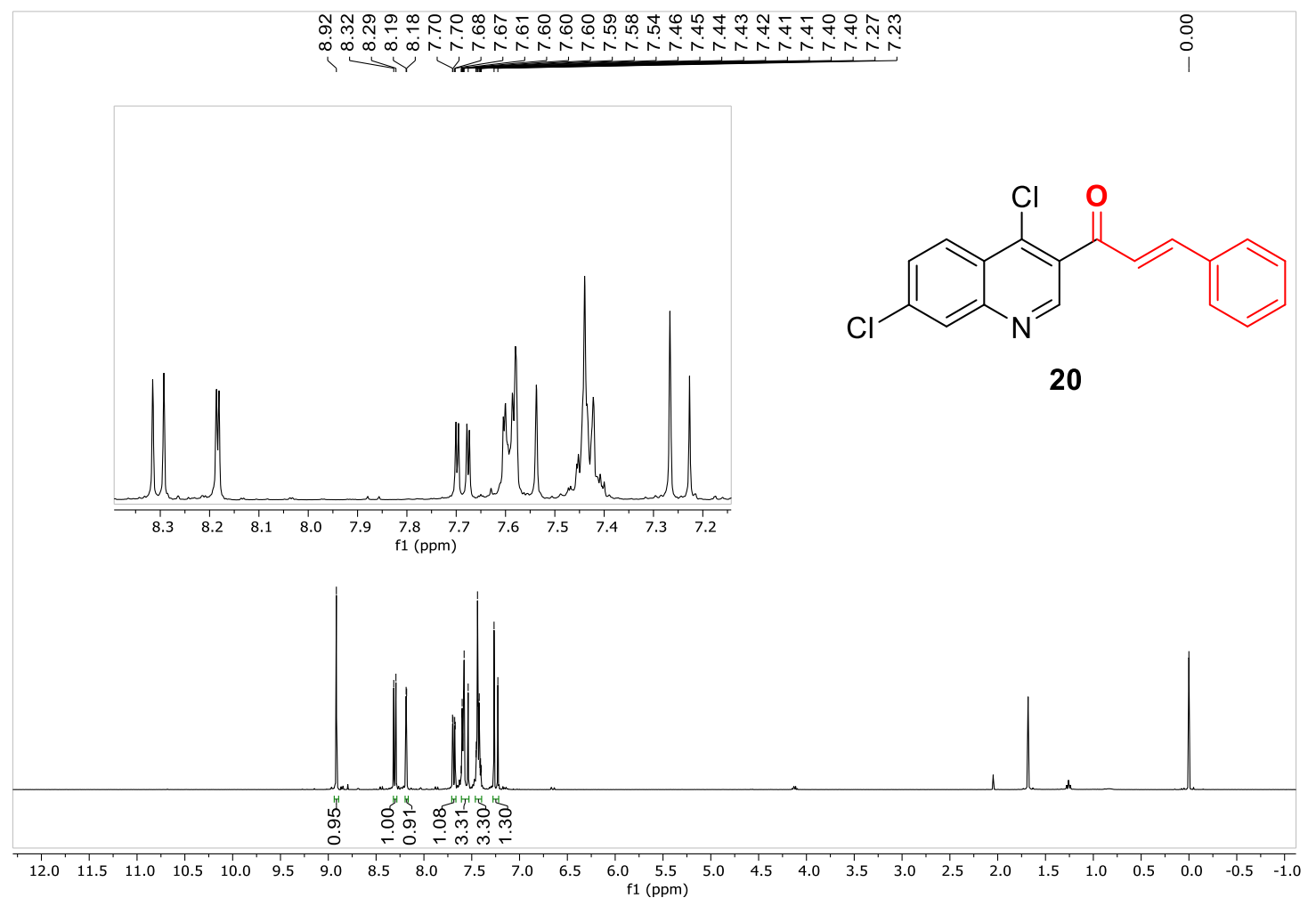

${ }^{13} \mathrm{C}\left\{{ }^{1} \mathrm{H}\right\}$ NMR spectrum in $\mathrm{CDCl}_{3}$ of compound $\mathbf{2 0}$

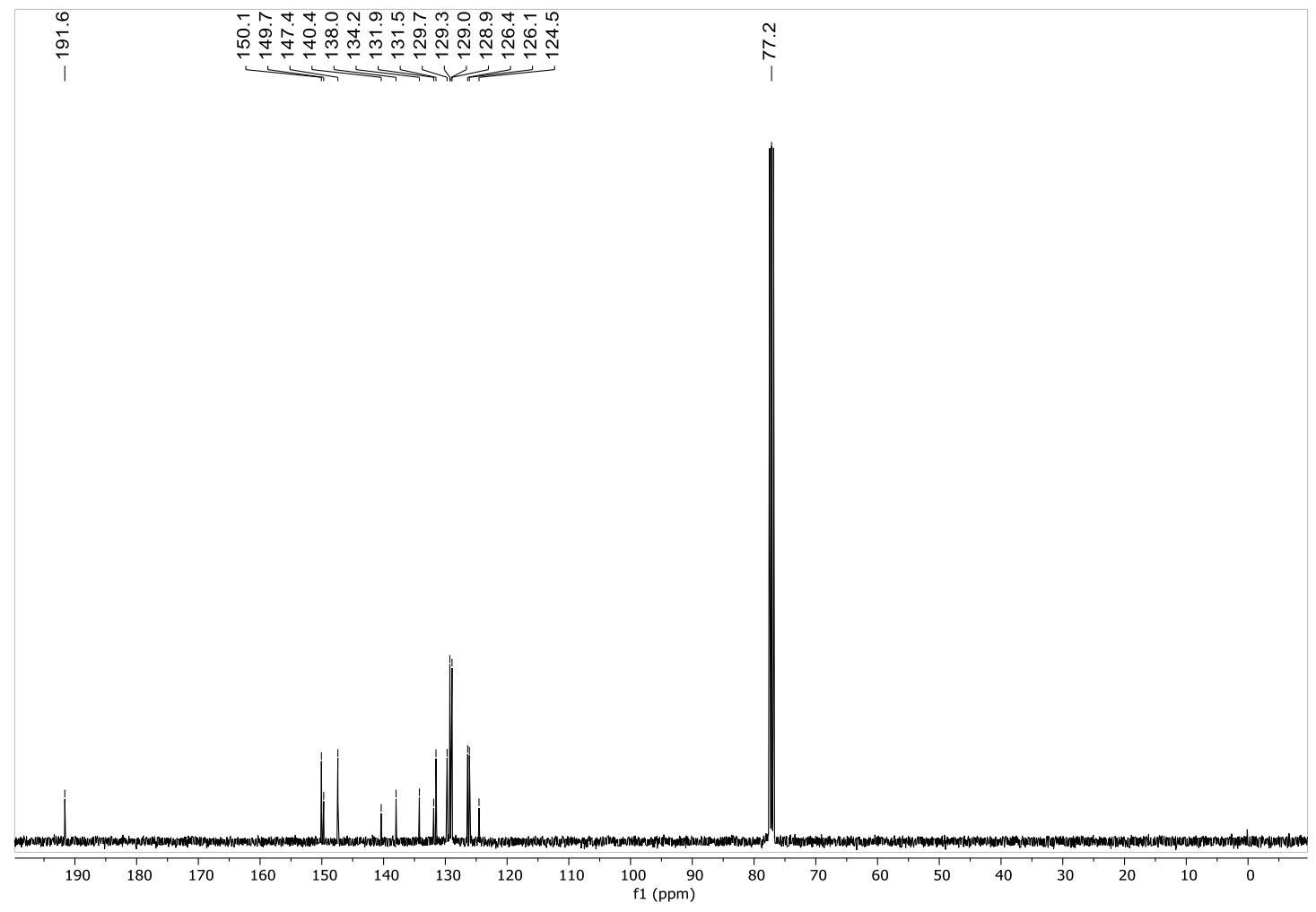

S65 
${ }^{1} \mathrm{H}$ NMR spectrum in $\mathrm{CDCl}_{3}$ of compound $\mathbf{2 4 a}$

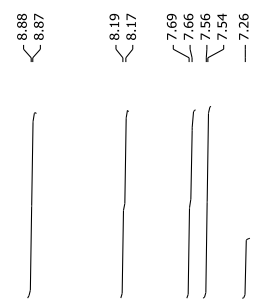<smiles>Clc1ccc2c(Cl)ccnc2c1I</smiles>

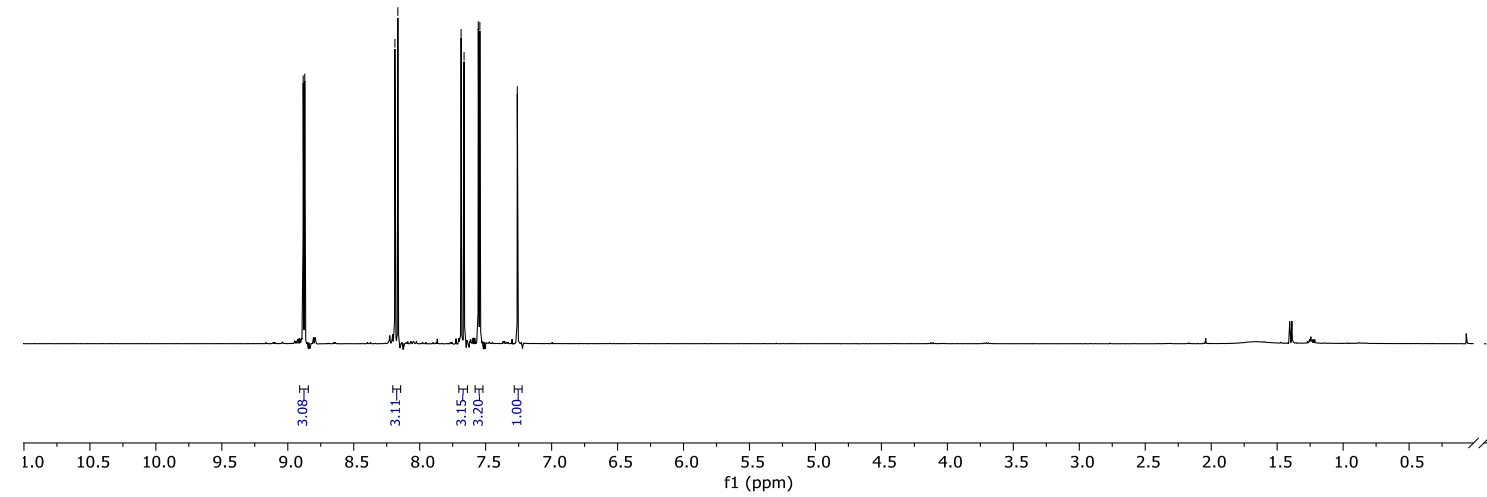

${ }^{13} \mathrm{C}\left\{{ }^{1} \mathrm{H}\right\} \mathrm{NMR}$ spectrum in $\mathrm{CDCl}_{3}$ of compound $\mathbf{2 4 a}$

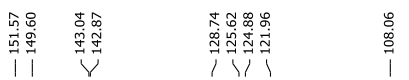

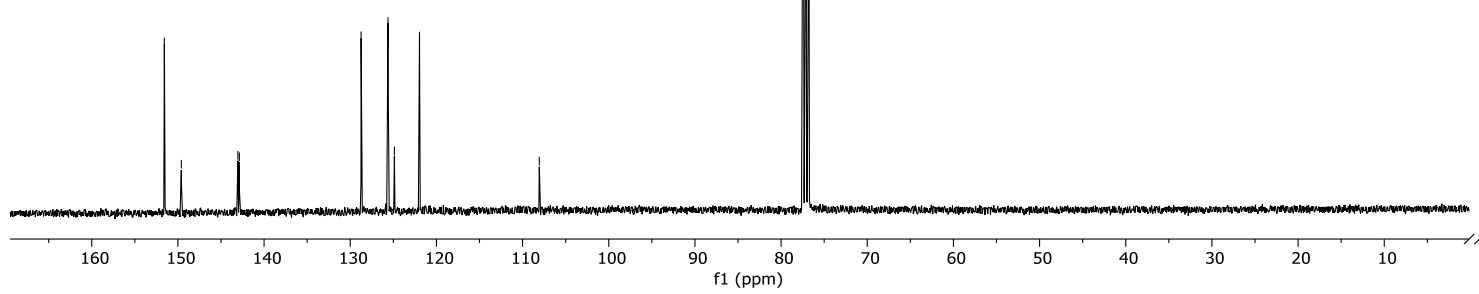


${ }^{1} \mathrm{H}$ NMR spectrum in $\mathrm{CDCl}_{3}$ of compound 24b

Y

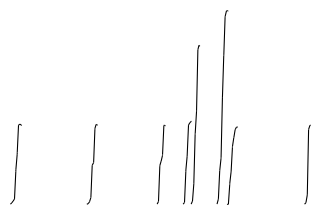<smiles>OC(c1ccccc1)c1c(Cl)ccc2c(Cl)ccnc12</smiles>

24b

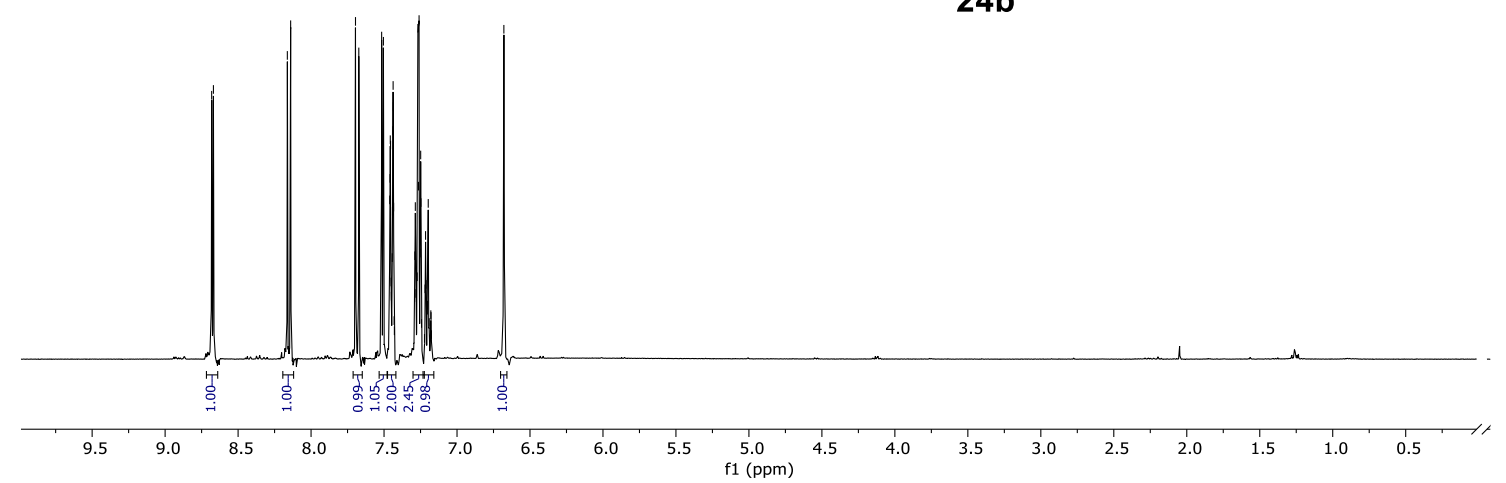

${ }^{13} \mathrm{C}\left\{{ }^{1} \mathrm{H}\right\}$ NMR spectrum in $\mathrm{CDCl}_{3}$ of compound $\mathbf{2 4 b}$

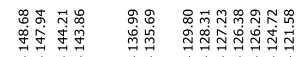

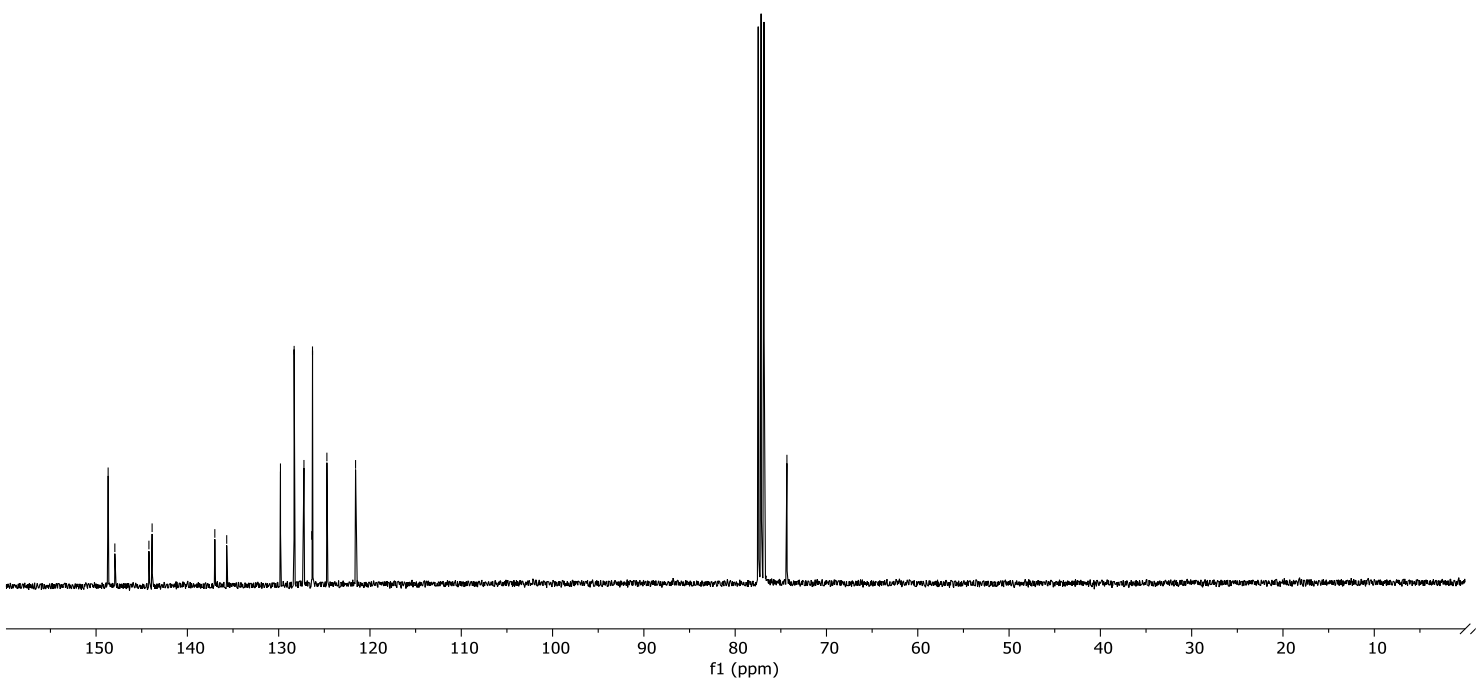


${ }^{1} \mathrm{H}$ NMR spectrum in $\mathrm{CDCl}_{3}$ of compound $\mathbf{2 4 c}$

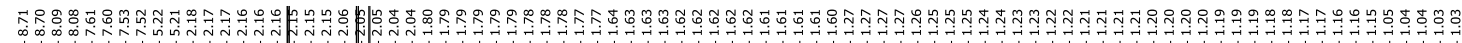

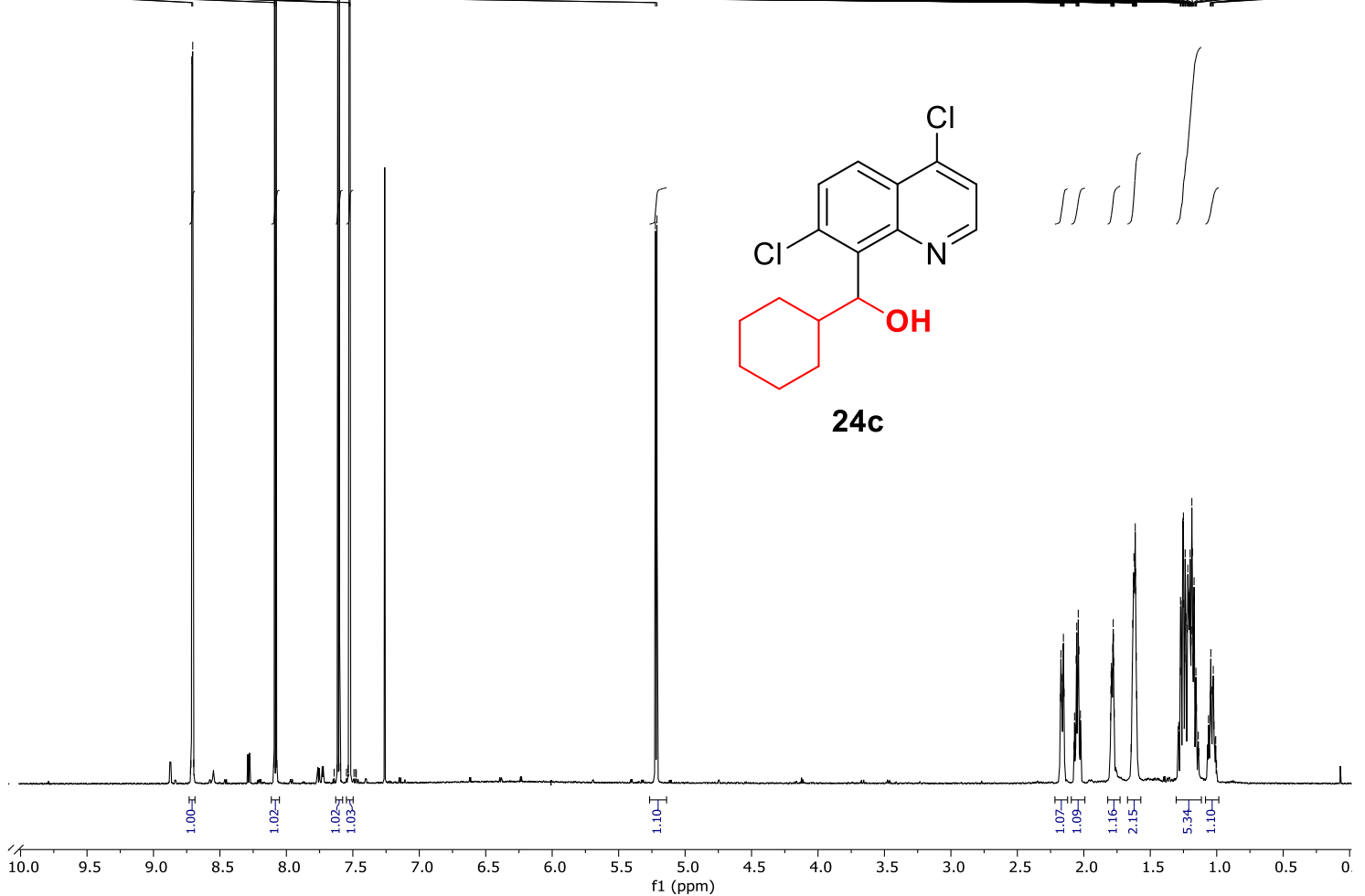

${ }^{13} \mathrm{C}\left\{{ }^{1} \mathrm{H}\right\} \mathrm{NMR}$ spectrum in $\mathrm{CDCl}_{3}$ of compound $24 \mathrm{c}$

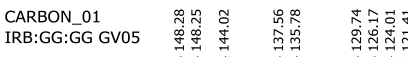

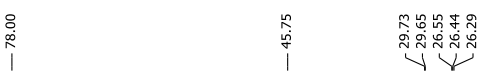

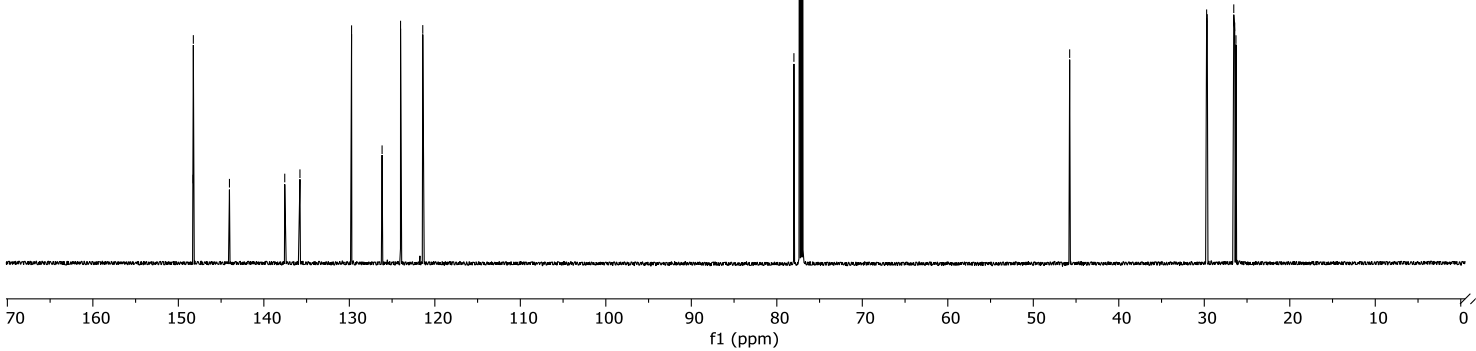


${ }^{1} \mathrm{H}$ NMR spectrum in $\mathrm{CDCl}_{3}$ of compound $\mathbf{2 4 d}$

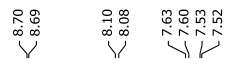

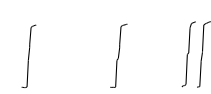<smiles>CC(C)C(O)c1c(Cl)ccc2c(Cl)ccnc12</smiles>

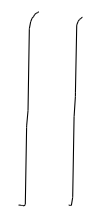

24d

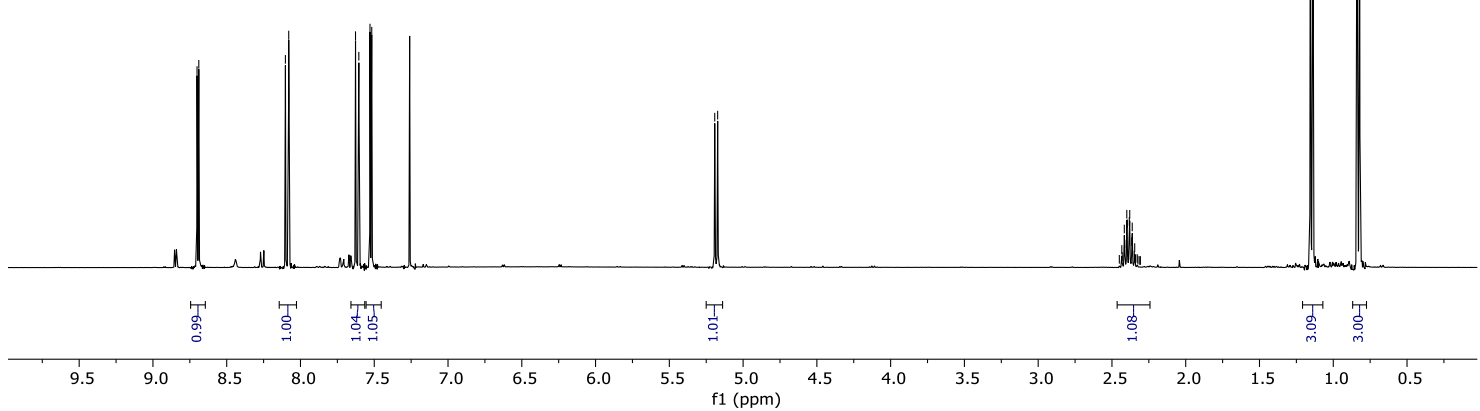

${ }^{13} \mathrm{C}\left\{{ }^{1} \mathrm{H}\right\}$ NMR spectrum in $\mathrm{CDCl}_{3}$ of compound $\mathbf{2 4 d}$

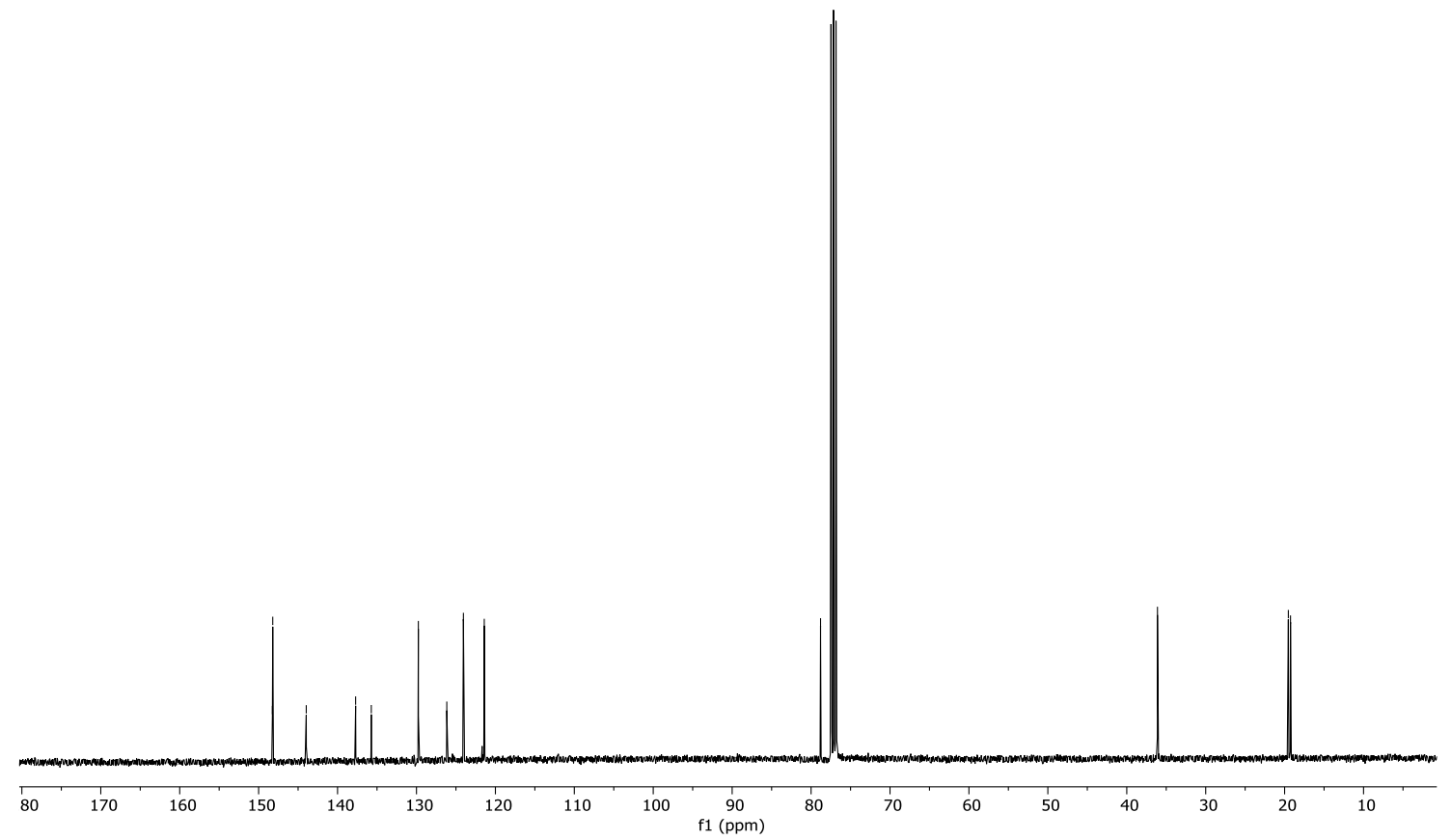


${ }^{1} \mathrm{H}$ NMR spectrum in $\mathrm{CDCl}_{3}$ of compound $\mathbf{2 4 e}$

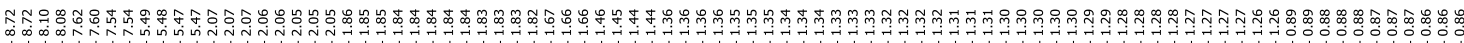

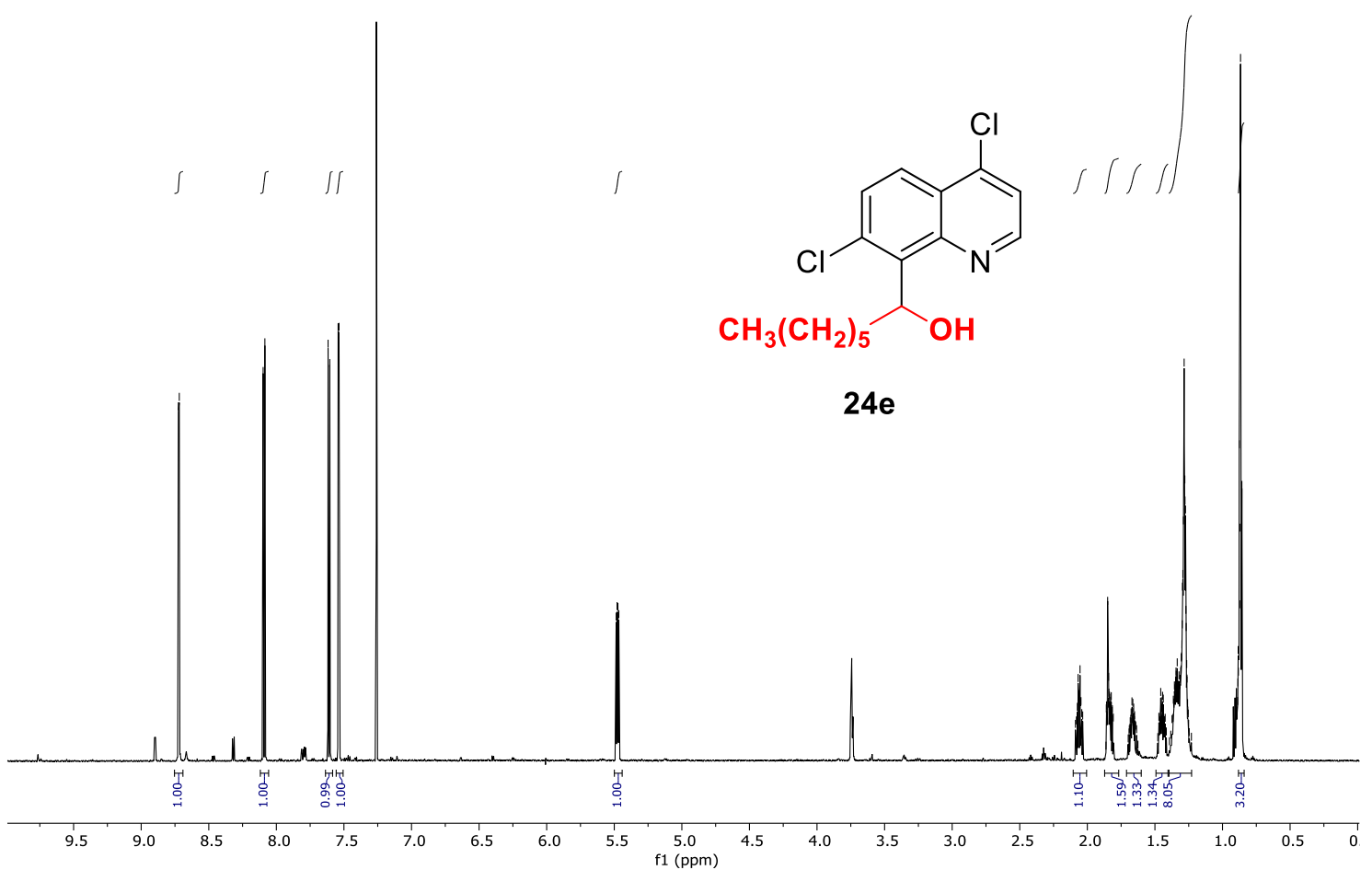

${ }^{13} \mathrm{C}\left\{{ }^{1} \mathrm{H}\right\}$ NMR spectrum in $\mathrm{CDCl}_{3}$ of compound $24 \mathrm{e}$

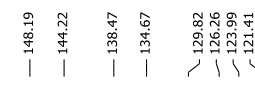

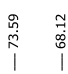

|

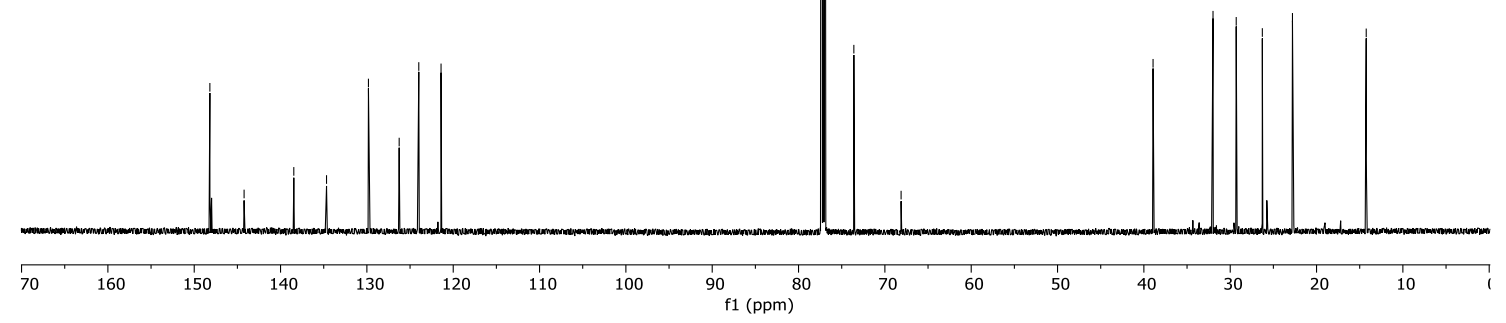


${ }^{1} \mathrm{H}$ NMR spectrum in $\mathrm{CDCl}_{3}$ of compound $\mathbf{2 4 f}$

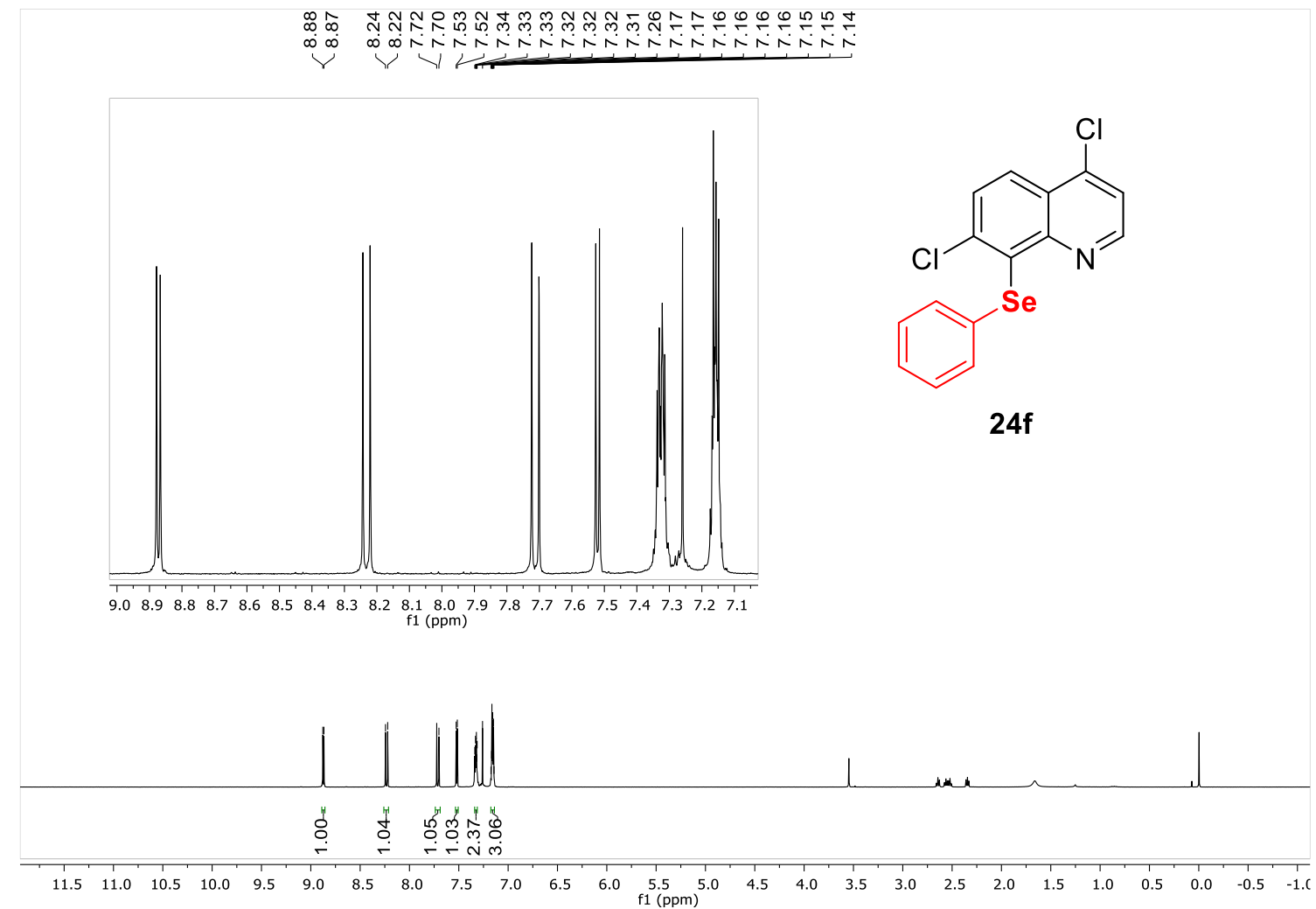

${ }^{13} \mathrm{C}\left\{{ }^{1} \mathrm{H}\right\}$ NMR spectrum in $\mathrm{CDCl}_{3}$ of compound $\mathbf{2 4 f}$

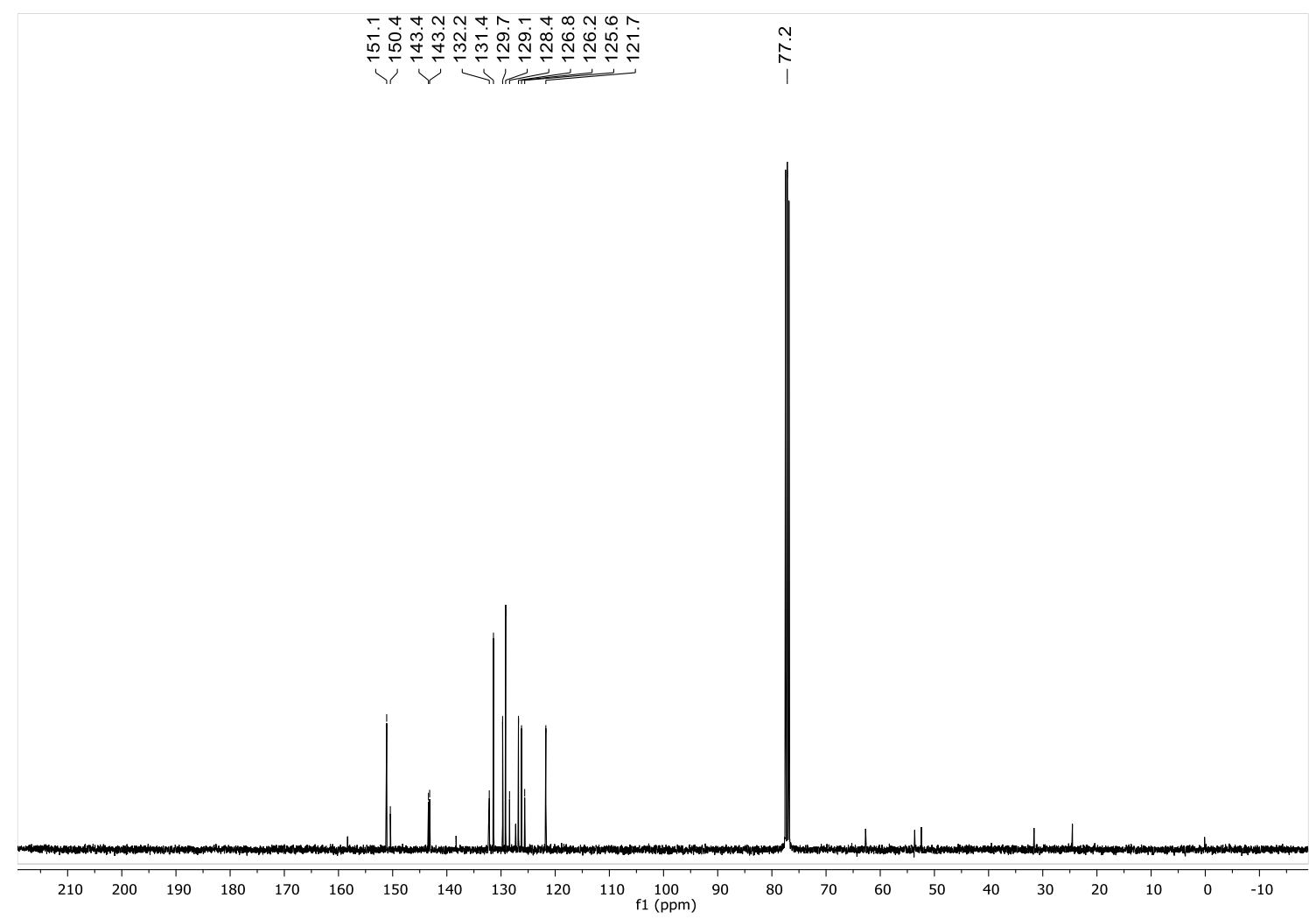


${ }^{1} \mathrm{H}$ NMR spectrum in $\mathrm{CDCl}_{3}$ of compound $\mathbf{2 4 g}$

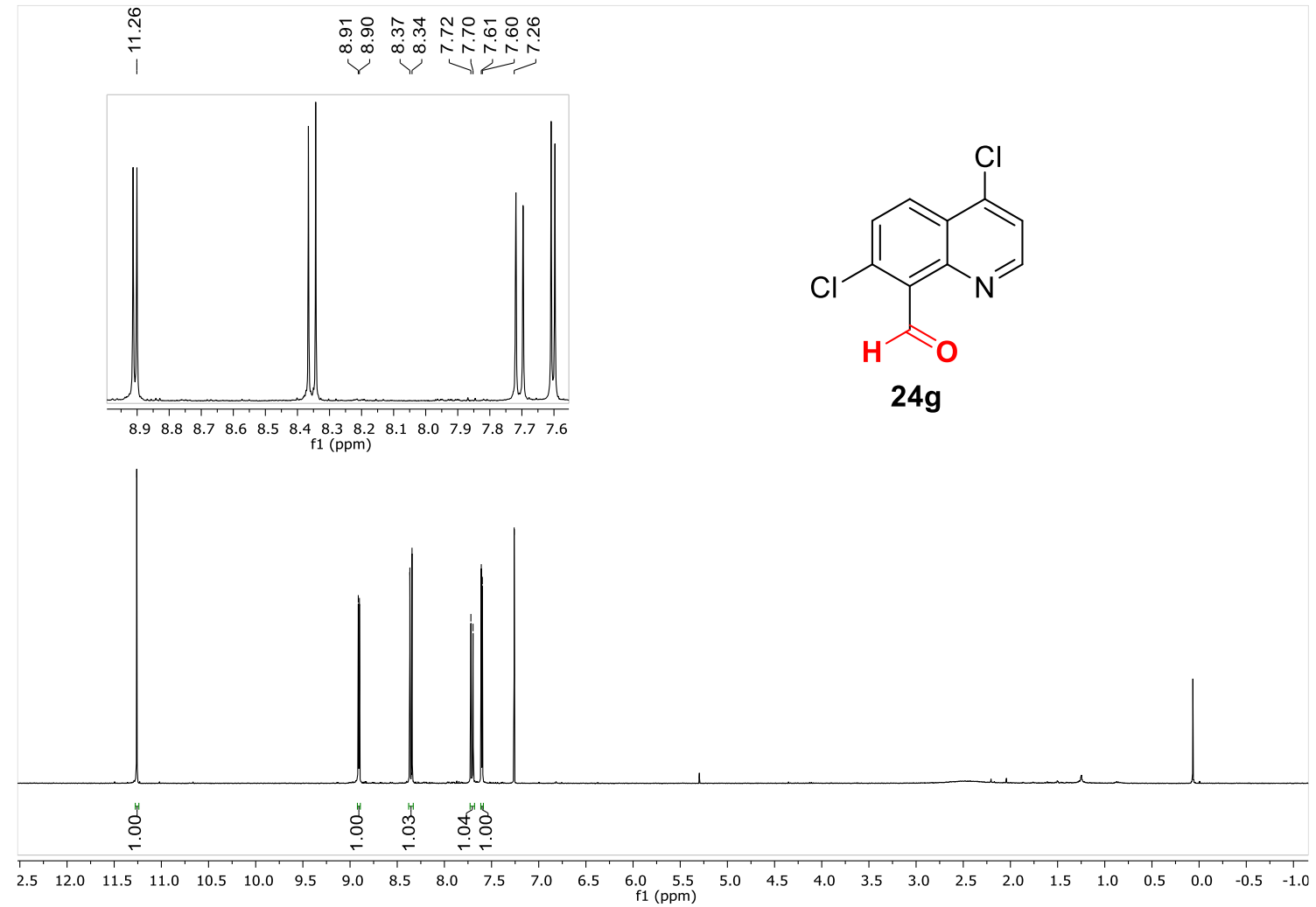

${ }^{13} \mathrm{C}\left\{{ }^{1} \mathrm{H}\right\}$ NMR spectrum in $\mathrm{CDCl}_{3}$ of compound $\mathbf{2 4} \mathbf{g}$

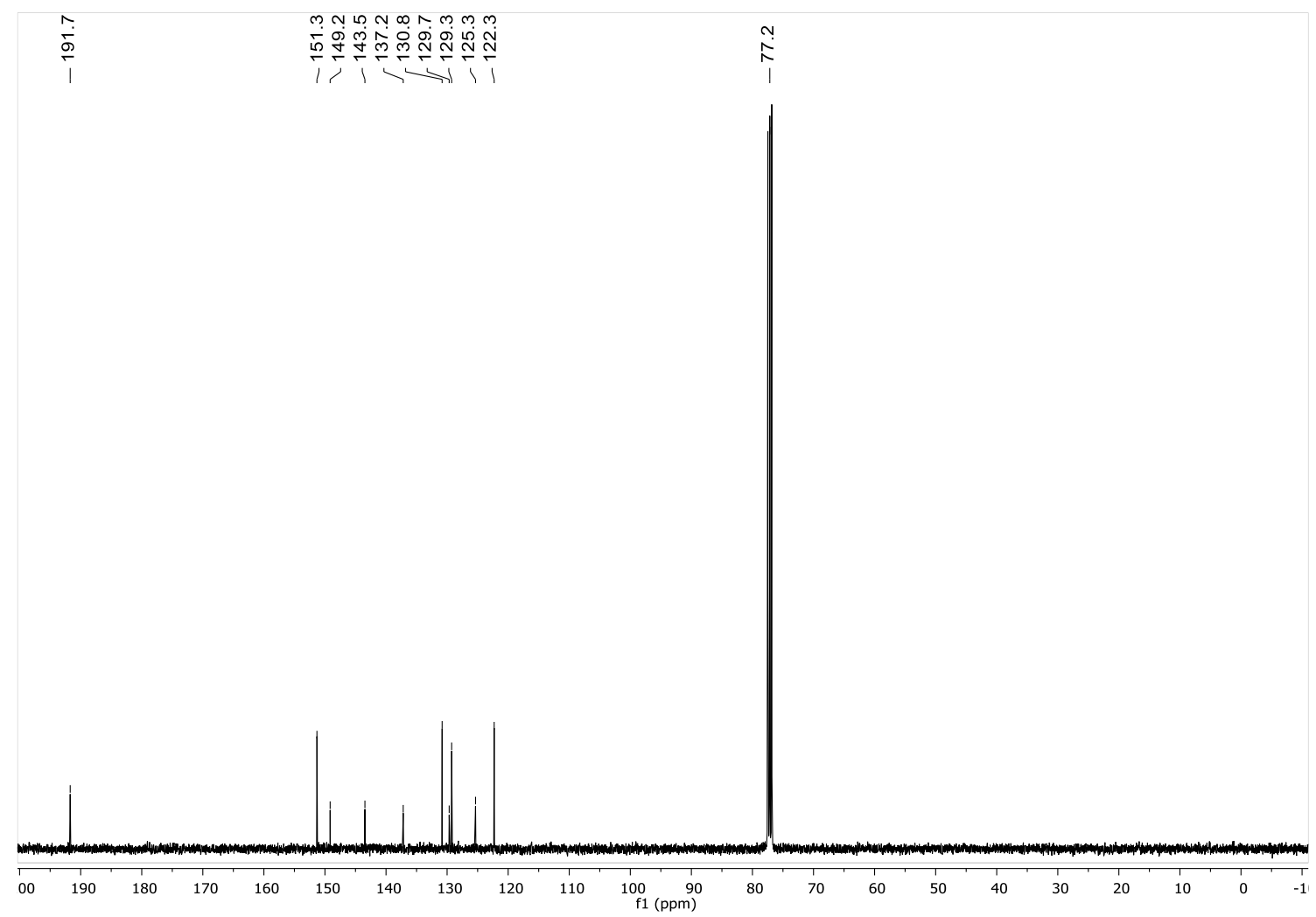


${ }^{1} \mathrm{H}$ NMR spectrum in $\mathrm{CDCl}_{3}$ of compound $\mathbf{2 4 h}$

గొ

Y

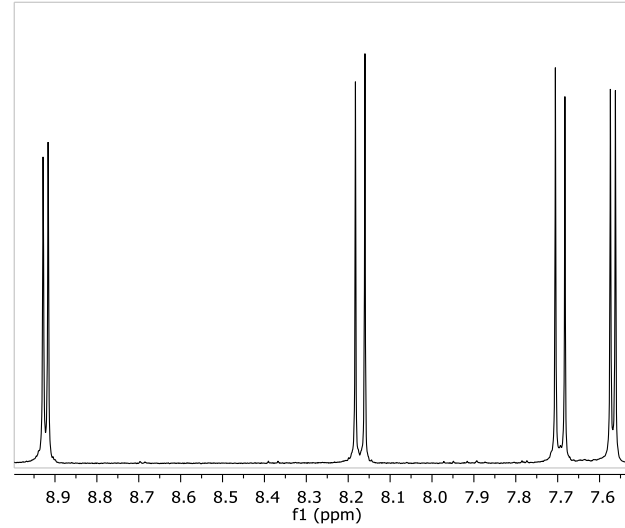<smiles>Clc1ccc2c(Cl)ccnc2c1Br</smiles>

24h

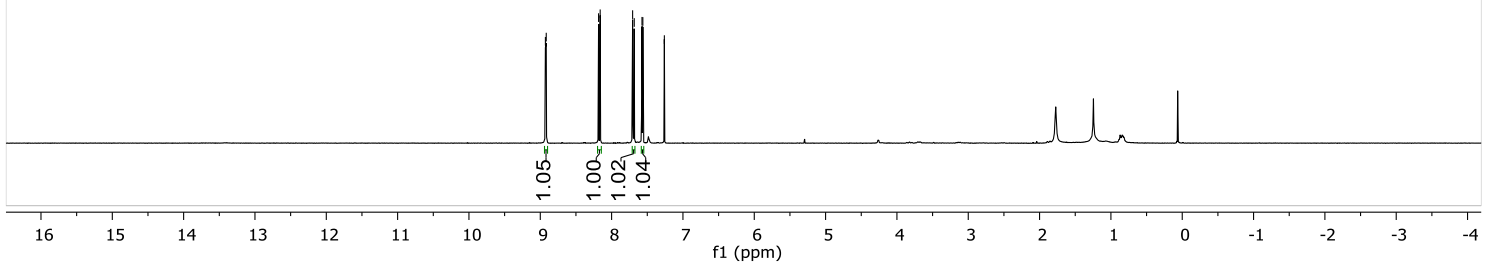

${ }^{13} \mathrm{C}\left\{{ }^{1} \mathrm{H}\right\}$ NMR spectrum in $\mathrm{CDCl}_{3}$ of compound $\mathbf{2 4 h}$

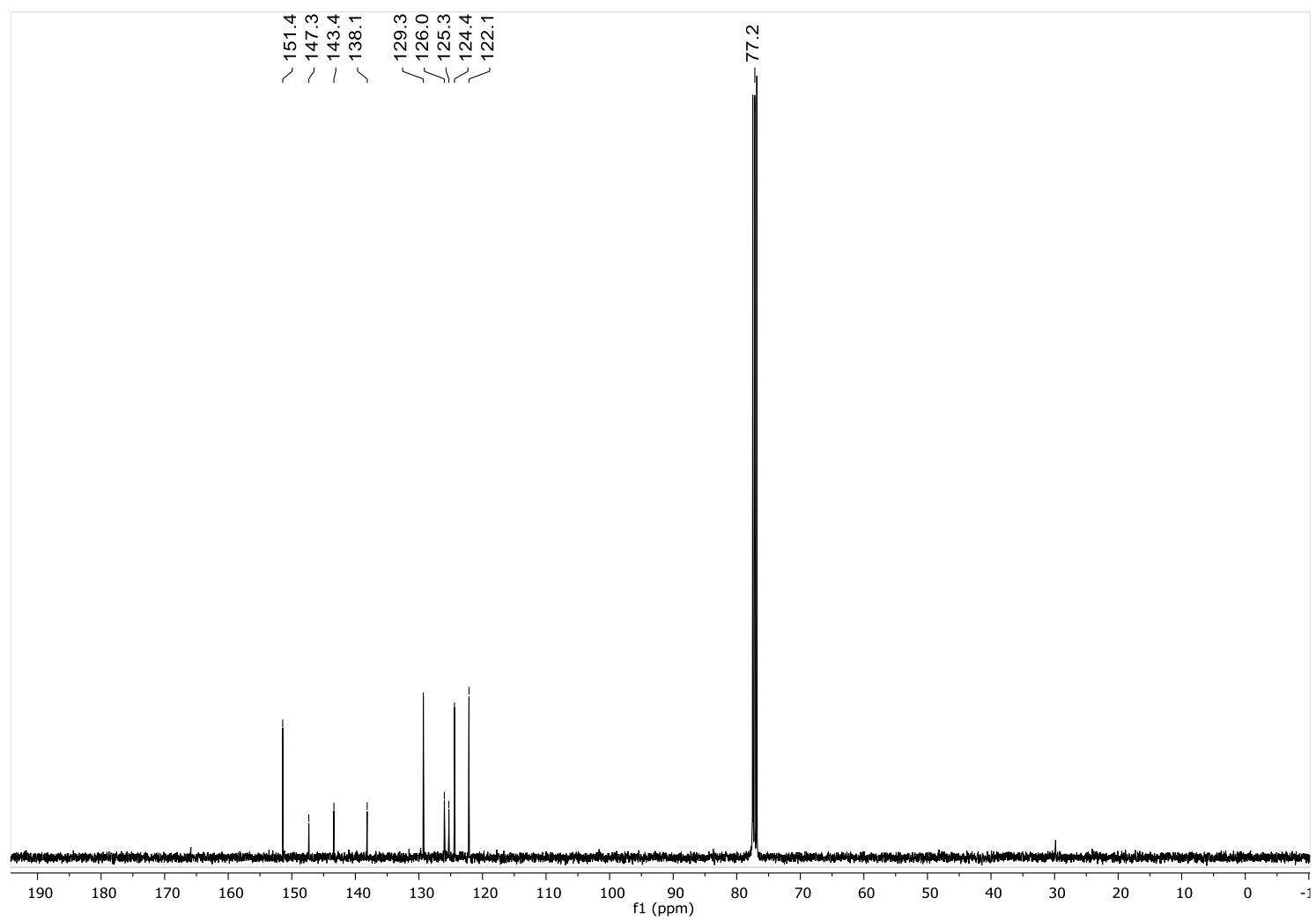

